\title{
Characterization of distinct contrast- and luminance-sensitive pathways in the Drosophila visual system
}

\author{
Dissertation \\ for the award of the degree \\ "Doctor rerum naturalium" (Dr.rer.nat.) \\ of the Georg-August-Universität Göttingen
}

within the doctoral program Sensory and Motor Neuroscience

of the Georg-August University School of Science (GAUSS)

submitted by

Katja Sporar

from Ljubljana, Slovenia

Göttingen, 2019 


\section{Thesis Committee}

Prof. Dr. Marion Silies

Institute of Developmental Biology and Neurobiology,

Johannes Gutenberg-Universität Mainz, Mainz

Prof. Dr. Martin Göpfert

Department of Cellular Neurobiology,

Schwann-Schleiden Research Centre, Georg-August-Universität, Göttingen

Prof. Dr. Nils Brose

Department of Molecular Neurobiology,

Max Planck Institute for Experimental Medicine, Göttingen

\section{Members of the Examination Board}

Reviewer:

Prof. Dr. Marion Silies

Institute of Developmental Biology and Neurobiology,

Johannes Gutenberg-Universität Mainz, Mainz

Second Reviewer: $\quad$ Prof. Dr. Martin Göpfert

Department of Cellular Neurobiology,

Schwann-Schleiden Research Centre, Georg-August-Universität,

Göttingen

\section{Further members of the Examination Board:}

Prof. Dr. Andreas Stumpner

Department of Cellular Neurobiology, Schwann-Schleiden Research Centre, GeorgAugust-Universität, Göttingen

Dr. Jan Clemens

Neural Computation and Behavior, European Neuroscience Institute, Göttingen

Prof. Dr. Gregor Bucher

Department of Developmental Biology, Georg August University Göttingen, Göttingen

Date of the oral examination: August 28 ${ }^{\text {th }}, 2019$ 



\section{Table of Contents}

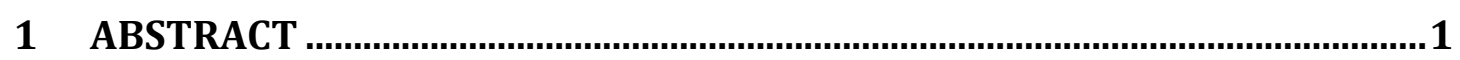

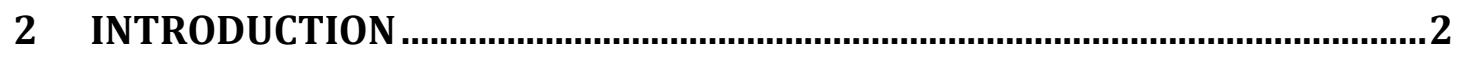

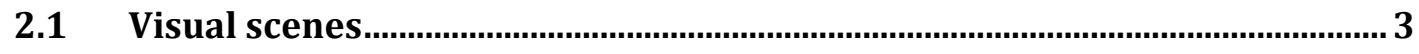

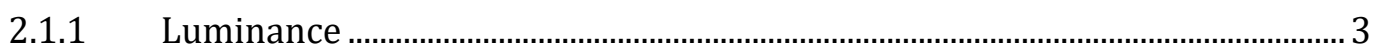

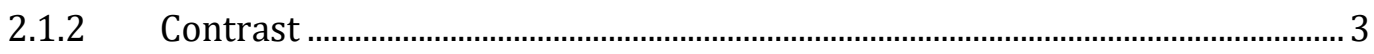

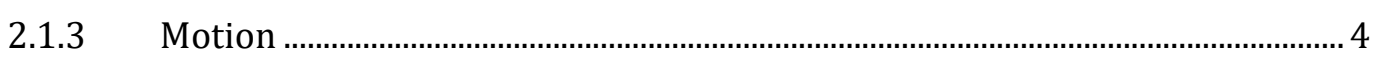

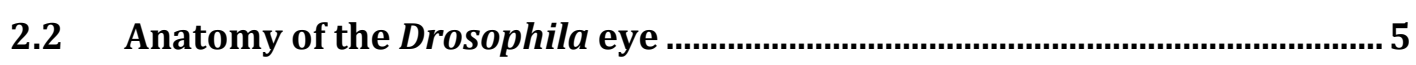

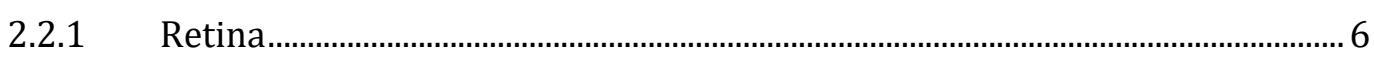

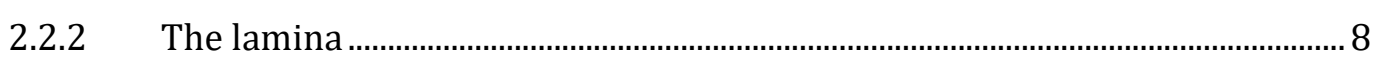

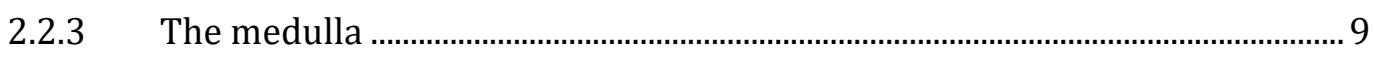

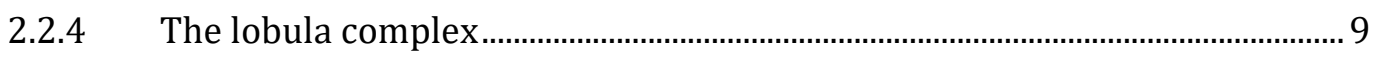

2.3 Processing of visual information in Drosophila .........................................10

2.3.1 Photoreceptors .................................................................................................. 10

2.3.2 Lamina Monopolar Cells (LMC) …………….................................................. 11

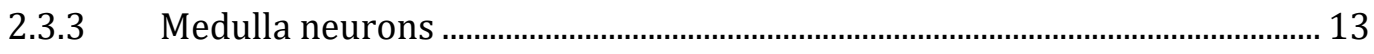

2.3.4 Neurons in the Lobula complex ........................................................................ 14

2.4 Vision in Changing Light conditions .........................................................

2.4.1 Visual adaptation ............................................................................................ 16

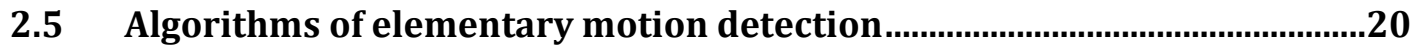

2.6 Drosophila tools to manipulate and monitor neuronal activity...................22

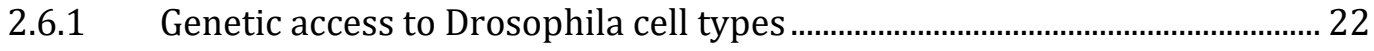

2.6.2 Monitoring neuronal activity …………………................................................. 23

2.6.3 Genetic manipulation of neural activity …………………………………....... 26

2.6.4 Altering gene expression .................................................................................. 26

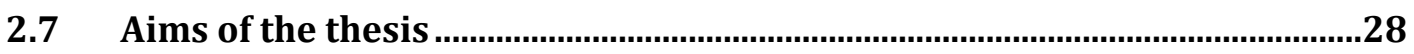

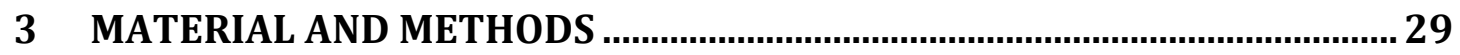

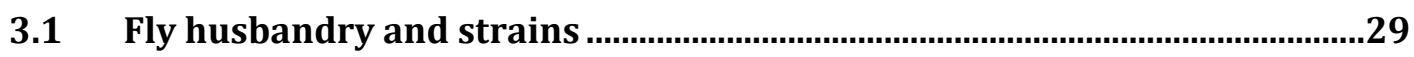

3.1.1 Drosophila strains used in this study ……..................................................... 29

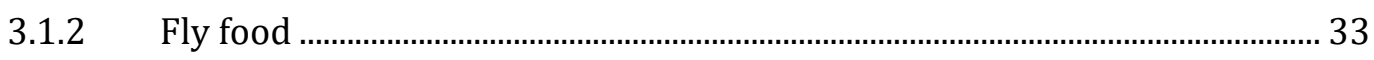

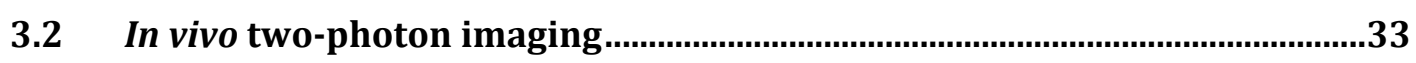

3.2.1 Fly preparation .............................................................................................. 33

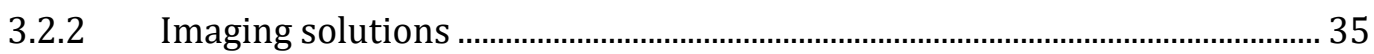


3.2.3 Two-photon imaging 36

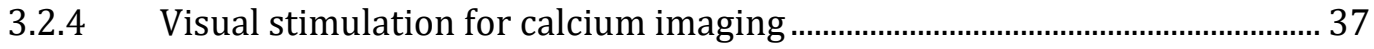

3.2.5 Two-photon calcium imaging analysis and statistical tests........................... 40

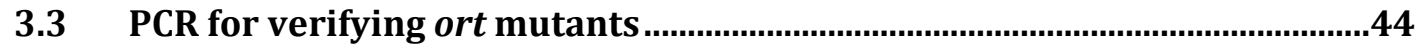

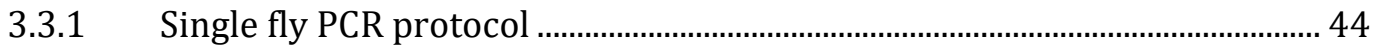

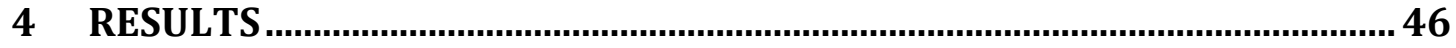

4.1 Contrast and luminance sensitivity of L2 and L3 ..........................................

4.1.1 L2 and L3 neurons show different physiological properties.......................... 46

4.1.2 L2, but not L3, calcium signals are contrast-sensitive ................................... 47

4.1.3 L3 is luminance sensitive and particularly active in dim light ..................... 50

4.1.4 L3 neurons carry contrast information in their fast voltage response ...... 57

4.1.5 L2 neurons are contrast-sensitive in a wide dynamic range of overall

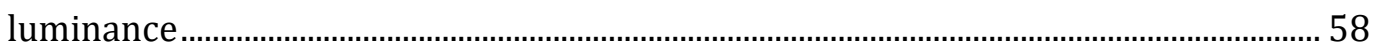

4.1.6 L3 is required in dim light relative to the adapted range. ............................. 61

4.2 Mechanisms that shape L2 and L3 physiology ...........................................63

4.2.1 L2 and L3 responses depend on the same major photoreceptor input.... 63

4.2.2 Contrast- and luminance-sensitive pathways depend on distinct photoreceptor-to-lamina neuron transformations........................................................... 66

4.2.3 R1-R6 photoreceptors show sustained responses to flashes......................... 71

4.2.4 L2 amplifies contrast information and discards luminance information from photoreceptors in a circuit-dependent manner ........................................................ 74

4.2.5 L3 properties do not depend on circuit interactions...................................... 80

4.2.6 L3 physiological properties are established at its postsynaptic site and

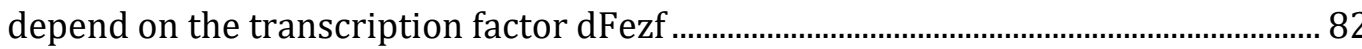

4.2.7 Preliminary study to identify molecular mechanisms that shape L2 and L3 physiology

\subsection{Integration of contrast and luminance sensitive responses in}

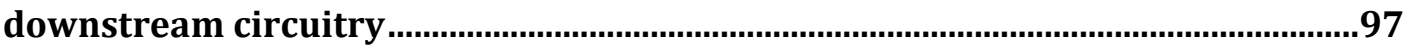

4.3.1 Tm9 neurons receive major input from L3 …………………………………... 97

5 DISCUSSION

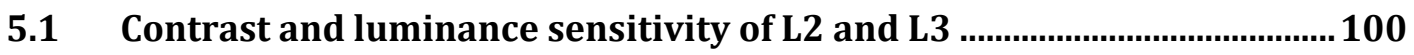

5.1.1 Luminance information is retained past photoreceptor cells......................100

5.1.2 Phasic and tonic responses are present in different sensory systems .....101

5.1.3 A role for luminance in image processing might be an evolutionary conserved feature 
5.1.4 Luminance information as an advantage for animals living in certain

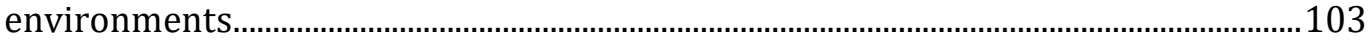

5.2 Mechanisms that shape L2 and L3 physiology ............................................ 105

5.2.1 Sustained R1-R6 calcium responses are sufficient for normal L2 and L3

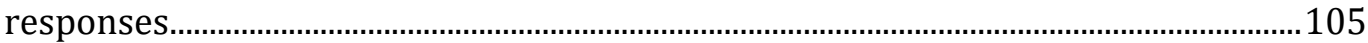

1.1.1. L2 elimination of a baseline is circuit-dependent ........................................106

1.1.1. Luminance-sensitivity of L3 is regulated by a single L3-specific transcription factor

\subsection{Integration of luminance and contrast information downstream of the}

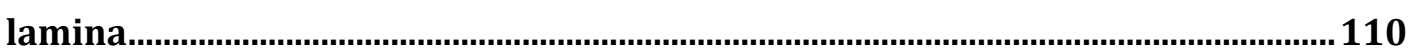

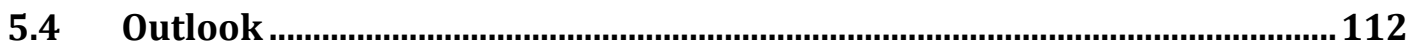

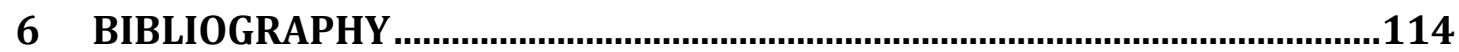

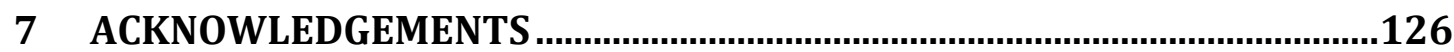





\section{ABSTRACT}

Visual systems are capable of extracting relevant information from dynamically changing environments. This allows animals to locate objects, escape predators and collisions or estimate self-motion, among others. During the day, illumination of the environment undergoes big intensity changes. Visual systems of many animals have evolved to cope with these changing light conditions by adapting to the input, which allows animals to perform well in bright daylight as well as at dusk and dawn, or when the world is fast changing e.g. due to self motion. This is true because visual systems ignore background illumination by computing contrast. Downstream of photoreceptors lamina monopolar cells (LMCs) were thought to discard luminance information and amplify photoreceptor contrast signals (Laughlin, 1989; Laughlin et al., 1987). Here we show that in the visual system of Drosophila, luminance information is retained past photoreceptors. We show that two distinct OFF-pathway inputs, the two lamina neurons L2 and L3, located downstream of photoreceptor cells, are contrast- and luminancesensitive, respectively. To understand what is shaping these early differences in visual processing, we tested the contribution of different photoreceptors inputs, the effect of lateral circuit inputs, as well as cell-autonomous differences. We show that L2 as well as L3 receive predominant input from R1-R6 and that two different types of photoreceptor-to-lamina transformations occur between the outer photoreceptors and L2 or L3. Contrast sensitivity of L2 is achieved by a circuit-dependent elimination of baseline, whereas luminance sensitivity of L3 depends on a cell-autonomous process that requires the L3-specific transcription factor, dFezf. We furthermore show that contrast- and luminance-sensitive information are combined in visual circuitry postsynaptic to lamina neurons. This suggests the importance of retaining the peripheral visual feature, luminance, in image processing. 


\section{INTRODUCTION}

The ability to sense the presence of light dates back to the earliest forms of animals, however, it took a long time to evolve truly functional visual systems. These visual systems enable animals to orient, catch the pray, avoid predators, or interact with conspecifics, to state only a few examples. The eyes have to take up information from the environment and the brain has to process this visual information in order to guide appropriate behavioral responses. Besides that, the brain receives information coming from other sensory systems, such as auditory and olfactory system. This raises a question of how this information is implemented at the neuronal level and a lot of research has been performed in order to gain knowledge in how the relevant information is obtained from the environment so as to allow appropriate transformation from sensation to behavior. Many studies aimed to understand how the sensory input in neuronal circuits is being processed and vision is extensively studied sensory system in different animals and also in humans. One of the fascinating aspects of animal eyes is that they can reliably process visual information under a range of light conditions. Many visual systems are well suited to perform at dusk and dawn, in bright daylight, and under changing light conditions, which is crucial for the behavior and survival. The amount of photons available for vision is an important factor in determining what an animal is able to see and this has had one of the major affects on the evolution of animal eyes. Nocturnal and deep sea animals need to capture as many photons as available and thus have large eyes with bad resolution, whereas diurnal animals could evolve eyes with high spatial resolution because they are not limited by the amount of photons available. Sensitivity and spatial resolution of an eye are counter-dependent and eye design compromises between resolution and sensitivity.

Adaptation of the visual system, initiated in the photoreceptor cells, allows an animal to see a fully detailed scene in the same way irrespective of the current light conditions. In other words, irrespective of background illumination, visual perception scales with the visual stimulus change, or contrast. Therefore, it is thought that neurons downstream of photoreceptor cells encode contrast while discarding luminance information (Laughlin, 1989). However, physiologically different types of LMCs have been described (Hardie and Weckström, 1990).

Although the eye morphology differs strongly across the animal kingdom, the principle of the basic visual information processing is very similar (Clark et al., 2014). Therefore, the general mechanisms of the visual processing can be studied Drosophila. 


\subsection{Visual scenes}

Sensory systems continuously receive constantly changing stimulations. For example, when an animal is navigating in its environment, the visual system and thus a receptive field of a single neuron is stimulated by an input of dynamically changing spatial patterns, luminances, contrasts and colors. Neurons must extract relevant information in order to correctly guide behavioral responses. Below I will describe the most fundamental components of the visual scene: luminance and contrast. Animals live in a constantly changing environment and regularly encounter moving objects, which cause changes in luminance over space and time. Thus, visual systems have evolved mechanisms to detect motion vision.

\subsubsection{Luminance}

Luminance is a measure of light coming from a light origin or from a reflecting object (Shapley and Enroth-Cugell, 1984). In order to obtain the distribution of local luminance within a given image, the luminance at each pixel has to be divided by the average luminance for the whole image. The distribution of luminance in a typical natural image is then obtained by combining the distributions across images and then scaling to the average luminance of the images (Geisler, 2008). This distribution is approximately normal on a logarithmic luminance axis and inclined toward the lower luminances (Brady and Field, 2000; Laughlin, 1981a).

\subsubsection{Contrast}

Contrast is used for discriminating objects from the background, recognizing shapes and patterns and for discriminating spatial configuration (Zeng et al., 2015). Contrast is a relative change of luminance. Luminance and contrast vary both within a given scene and across scenes and a lot of work has been done measuring the statistics of luminance and contrast within images of natural scenes (Geisler, 2008). Similar to luminance, contrast is positively skewed on a linear scale, meaning that in natural images there are more dark than light contrasts (Geisler, 2008; Ratliff et al., 2010). There are two main definitions of contrast: Weber and Michelson contrast.

Weber contrast is used when one would like to study the visibility of aperiodic and uniform objects on a certain background. The Weber contrast definition is:

$$
C=\left(\frac{I-I_{b}}{I_{b}}\right),
$$

where $I$ is the object luminance and $I_{b}$ is the background luminance. $I-I_{b}$ is sometimes called $\Delta I$, therefore the equation is usually written as: 


$$
C=\frac{\Delta_{I}}{I_{b}} .
$$

The second commonly used definition of contrast is Michelson contrast. Michelson contrast is used to describe the contrast of periodic spatial patterns such as sine gratings. The Michelson contrast definition is:

$$
C=\frac{I_{\max }-I_{\min }}{I_{\max }+I_{\min }}
$$

with $I_{\max }$ and $I_{\min }$ being the maximum and the minimum luminance (Michelson, 1972). Both Weber and Michelson definitions of the contrast are used in the literature of neuronal adaptation and it each of these two contrast definitions is appropriate for a particular kind of stimuli (Shapley and Enroth-Cugell, 1984).

Already 50 years ago, it was realized that contrast sensitivity is not stable but heavily dependent on the history of visual experience (Blakemore and Campbell, 1969). For example, cortical visually evoked potential amplitudes decrease during the presentation of sine wave gratings, a result of adaptation, and the response amplitudes recovered after a period of normal visual experience (Blakemore and Campbell, 1969). In addition, the effect of visual adaptation has been studied in invertebrates in great detail and it was shown that visual processing relies on contrast. Whereas photoreceptors responses encode both contrast and luminance, downstream neurons are thought to further amplify photoreceptor contrast signals while discarding information about constant illumination (Laughlin, 1989; Laughlin et al., 1987). This allows animals to encode contrast irrespective of the luminance. I will discuss neuronal adaptations in greater detail in Chapter 2.4.1.

\subsubsection{Motion}

Another fundamental feature of the visual scene is motion. Motion can be described as a shift in the position of an image. The majority of these image shifts on the animal retina are generated by the animal itself. Besides that, image shifts come also from the external world, for example from an approaching predator, moving conspecifics etc. (Cohen et al., 1977). Thus, visual motion is a fundamental cue for an animal. Moving objects cause spatiotemporal correlation in reflected light. In other words, moving objects cause changes in luminance over space and time and to compute motion, the intensity of individual points in space at one time has to be correlated closely with the intensity of the adjacent point in space at a later time (Yang and Clandinin, 2018). Photoreceptor cells themselves are not motion-sensitive but instead respond luminance changes. In order to assess the direction of motion, signals coming from at least two points in time 
and space need to be compared. Several algorithms have been proposed that suggest how this can be done, all of which are based on the idea of the spatially separated correlations in luminance changes, followed by temporal filtering and a non-linearity (Adelson and Bergen, 1985; Barlow and Levick, 1965; Hassenstein, 1951) (see 2.5 Algorithms of elementary motion detection).

\subsection{Anatomy of the Drosophila eye}

Drosophila has been a model organism to study vision for more than 80 years (Hecht and Wald, 1934; Kalmus, 1943). Fruit flies are a great model to study visual processing strategies, because one can study behavioral responses to motion, visual system anatomy is known down to single cell types and synaptic connections, and it is amenable to genetics to allow manipulating both cellular, neuronal function, as well as molecular mechanisms. Quantitative measurements of the fly's motion-guided behavioral responses began more than half a century ago (Buchner, 1976; Götz, 1964; Heisenberg and Wolf, 1984; Tammero and Dickinson, 2002). Below I will briefly describe the Drosophila optic lobe with a focus on neurons that are involved in motion detection. In brief, in Drosophila, visual information is passed from the retina where light hits the photoreceptor cells to the optic lobe consisting of three visual ganglia: the lamina, medulla and the lobula complex, composed of the lobula and the lobula plate (Figure 1AC). In the Drosophila optic lobe there are at least 113 different cell types (Fischbach and Dittrich, 1989). These data still provide a valuable insight for classification of the neurons in the fly visual system. They were subsequently complemented by genetic studies that added the description of further cell types (e.g. Nern et al., 2015). Recently, electron microscopic reconstructions provided new insights on circuit connectivity, by mapping synaptic connections between known cell types (Rivera-Alba et al., 2011; Takemura et al., 2013, 2017).

A

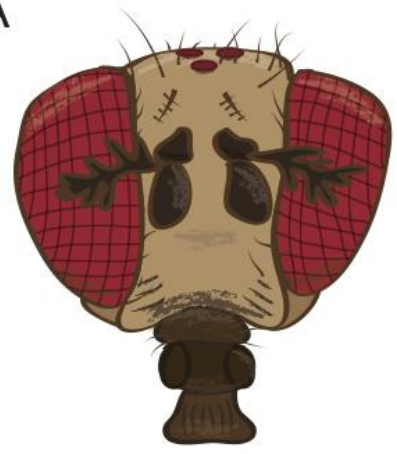

B

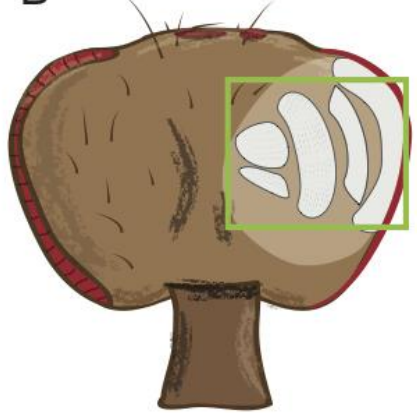

C

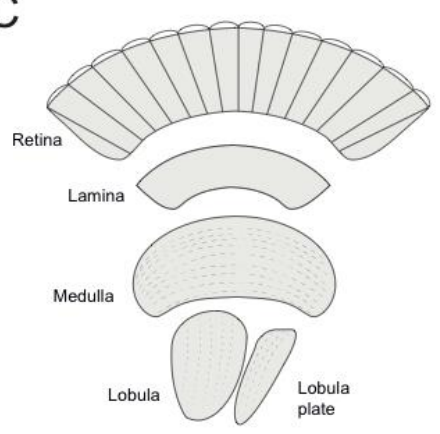


Figure 1: The fly visual system represents most of the brain.

(A-B) Schematics of the fly head shown from two different perspectives: front (A) and back (B). Green box illustrates the position of the optic lobe shown in (C). (C) Fly visual system schematic, showing the three optic ganglia.

\subsubsection{Retina}

"Fly's eye looks like a raspberry." These were the words written in the L'occhio della mosca, written by an Italian scientist Hodierna in 1644 (Bardell, 1993). This is true because the retina of a fruit fly is build of around 800 individual optic units, known as ommatidia, of which each unit consists of eight photoreceptor cells R1-R8, supporting cells and pigment cells (Kirschfeld, 1967). R1-R8 are arranged cylindrically, outer R1-R6 are located peripherally, and the central R7 and R8 are positioned in the middle with R7 distally and R8 proximally (Figure 2A). R1-R6 form an achromatic channel, involved in detection of motion and R7 and R8 feed into a color channel (Heisenberg and Buchner, 1977, Salcedo et al., 1999). Drosophila can sense a wide spectrum of light as it expresses six different rhodopsins, Rh1-Rh6. The photoreceptor cells sensitivity to different wavelengths is regulated by the expressed rhodopsin (Britt et al., 1993; Lin and Sakmar, 1999). R1-R6 cells contain a broad blue-sensitive Rh1 rhodopsin, R7 cells contain Rh3 and Rh4, which are both sensitive to UV and R8 cells contain rhodopsins Rh5 and Rh6, which show sensitivity to blue and green spectrum (Figure 2B-C) (Salcedo et al., 1999). Different expression of R7 and R8 rhodopsins leads to two types of ommatida: "pale" and "yellow". In Drosophila, there is a color-opponent processing of UV-short/blue in the R7/R8 terminals of "pale" and UV-long/green in the R7/R8 terminals of "yellow" ommatidia. In the same ommatidium, there is a mutual inhibition between the central photoreceptors (Schnaitmann et al., 2018).

A

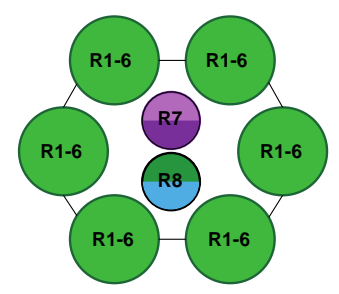

B

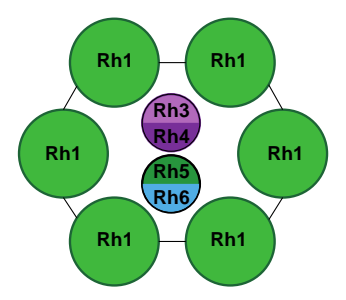

C

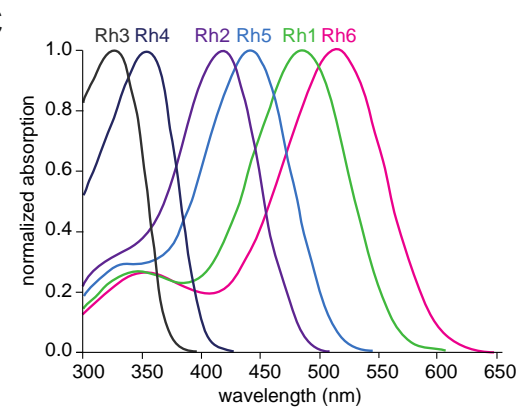

Figure 2. In Drosophila, opsins are differentially expressed.

(A) Schematic showing an arrangement of photoreceptor cells in a cross-section perspective. R1-6, are located peripherally, and the central photoreceptor cells, R7 and R8, are positioned in the ommatidium center. (B) Schematic of the differential rhodopsin expression in different types of photoreceptor cells. (C) $\mathrm{Rh} 1$ has an absorption maximum at $480 \mathrm{~nm}, \mathrm{Rh} 2$ at 420, Rh3 maximally absorbs at 331, Rh4 at 335, Rh5 at 
442 and Rh6 at $515 \mathrm{~nm}$. (A) and (B) modified after (Jackowska et al., 2007). Schematic in (C) was modified after (Stavenga and Arikawa, 2008).

Photoreceptors project their terminals to the optic lobe. R1-R6 axon terminates in the lamina, while R7-8 photoreceptor axon terminals terminate in the medulla (Fischbach and Dittrich, 1989). Photoreceptor from different ommatidia project to their targets such that one cartridge in the lamina corresponds to one point in the space in the visual field, thus forming a retinotopic map (review Clandinin and Zipursky, 2002). This retinotopic map is preserved in subsequent ganglia, despite the fact that this map is inverted twice around the anterior to posterior axis. First, there is a lamina-medulla inversion and a second one is medulla-lobula complex inversion (Fischbach and Dittrich, 1989).

In all animals, vision depends on visual pigments or opsins and more than 1000 opsins have been identified (Yau and Hardie, 2009). The arrangement of the pigmentcontaining membranes is different between vertebrates and invertebrates, while opsins are very similar (Abrahamson et al., 1974). Most animal opsin-based pigments are Gprotein-coupled receptors (GPCRs), consisting of a chromophore and an opsin (Terakita, 2005; Vogt and Kirschfeld, 1984; Yau and Hardie, 2009). The photosensitive structure of the invertebrates is the so-called rhabdomere, an array of microvilli, while in the vertebrates, the flattened membrane vesicles, or disks, are positioned in the rod outer segment. Besides the different arrangement of the pigment membranes, there is also a difference in the response to illumination. Illumination causes a hyperpolarization in vertebrate cells, while invertebrate photoreceptors depolarize. Photoreception begins with a photon of light being absorbed by an opsin (Heintzen, 2012). In invertebrates, upon absorption of light, the chromophore undergoes a conformational change, which leads to an active form of the rhodopsin, the so-called metarhodopsin (Hardie, 2012; Montell, 2012). This chromophore conformational change causes a cascade of events, resulting in products inositol 1,4,5-trisphosphate (InsP3) and diacylglycerol (DAG). This drives the activation of the calcium-permeable channels called 'trp' and 'trp-like' and this leads to the calcium influx and membrane depolarization (Hardie, 2012).

Throughout the animal kingdom, photoreceptors capture photons and signal light information to the nervous system. Photoreceptors are diverse in design and purpose between invertebrates and vertebrates. However, phototransduction, the mechanism by which absorbed photons are transformed into an electrical response, is highly conserved as it is based in all cases on a single class of photoreceptors, the opsins (review Yau and Hardie, 2009). 


\subsubsection{The lamina}

Photoreceptors project into the lamina, the first optic neuropile. The lamina is organized in stereotypically arranged cartridges. Each of the around 800 retinotopically arranged cartridges in the lamina corresponds to a discrete visual field of view $\sim 5^{\circ}$ (Braitenberg, 1967) and each cartridge houses 12 neuronal cell types and includes 5 large monopolar cells (also known as lamina monopolar cells or LMCs), 6 putative feedback neurons as well as a lamina intrinsic cell (Lai) (Figure 3A) (Fischbach and Dittrich, 1989). These neurons are either columnar neurons, i.e. in each retinotopic column there is one cell, or multicolumnar neurons, which span multiple columns. The columnar neurons include the LMCs, L1-L5, and T1, C2 and C3. Lai, lamina wide-field neurons Lawf1 and Lawf2, and the lamina tangential cell, Lat, are multi-columnar neurons (Fischbach and Dittrich, 1989). LMCs can be characterized by their different distributions of the dendrites and by their layer-specific axon terminal projection. L1 axon terminals project to the layer M1 and M5, L2 neurons project to the M2 layer, L3 to M3, L4 project to M2 and M4 layer and L5 neuron axon terminals project in M1, M2 and M5 layer in medulla (Figure 3A) (Fischbach and Dittrich, 1989; Meinertzhagen and O'Neil, 1991a; Takemura et al., 2008).

In every cartridge, at the level of photoreceptor terminals, four postsynaptic elements constitute a tetrad synapse (Frohlich and Meinertzhagen, 1982). Every R1-6 terminal synapse with four postsynaptic elements: L1, L2, amacrine cell and then either L3 or a glial cell. Thus, the LMCs that make direct synaptic contact with R1-6 are L1-L3, whereas L4 and L5 receive photoreceptor inputs indirectly (Meinertzhagen and O'Neil, 1991a; Nicol and Meinertzhagen, 1982; Rivera-Alba et al., 2011). In the lamina, L1 and L3 lack presynaptic sites, while L2 and L4 make synapses with each other, both within and between neighboring cartridges and with R1-R6 (Meinertzhagen and O'Neil, 1991a; Meinertzhagen and Sorra, 2001). In the medulla, C2 and C3 make strong synapses with L2 neurons (Meinertzhagen and O'Neil, 1991a), whereas L3 receives input only from C2 (Takemura et al., 2013). Tetrad synapses are sites of release of histamine, which is the main photoreceptor neurotransmitter (Hardie, 1987, 1989). Histamine directly activates postsynaptic chloride channels on LMCs (Hardie, 1987, 1989). There are two histaminegated channel genes ort and HisCl1 (Witte et al., 2002; Zheng et al., 2006). Ort is the native LMC synapse receptor, whereas HisCl1 can be found in R7 and R8 photoreceptors (Schnaitmann et al., 2018) and in glia cells, which shape LMC postsynaptic responses (Pantazis et al., 2008). It has recently been shown that there is direct mutual inhibition between central photoreceptors of one ommatidium type via HisCl1 and indirect inhibition mediated by Ort. All this mediates color-opponency in R7-R8 (Schnaitmann et 
al., 2018). Together, Ort is the channel directly expressed on LMCs and previous studies reported that ort mutants are motion blind (Joesch et al., 2010; Rister et al., 2007).

\subsubsection{The medulla}

Most lamina neurons send their axon terminals to the medulla, where the dendrites of transmedullary (Tm) and medulla intrinsic (Mi) dendrites are located, among other cell types. Retinotopy is maintained in medulla, as axons from each lamina cartridge project into a specific medulla column. The medulla is composed of 10 layers, M1-M10 and houses more than 70 different types of cells (Figure 3B) (Fischbach and Dittrich, 1989). Certain medulla cells, such as Mi neurons, project between different medulla layers, whereas other types, such as Tm neurons, connect the medulla and to downstream ganglia. As in the lamina, many neurons are columnar, whereas other cells connect the columns horizontally (Fischbach and Dittrich, 1989; Nern et al., 2015).

\subsubsection{The lobula complex}

Lobula and lobula plate are the next processing centers. Medulla neurons synapse onto T4 (ON) and T5 (OFF) dendrites (Fisher et al., 2015a; Maisak et al., 2013; Shinomiya et al., 2014; Takemura et al., 2013). T4 and T5 neurons come in four subtypes, with each subtype terminating in a distinct lobula plate layer (Figure 3C) (Shinomiya et al., 2014). Each T4/T5 subtype responds specifically to only one of the four cardinal directions of motion. All T4 / T5 subtypes make synapses with lobula plate tangential cells (LPTCs), which show direction-selective responses to motion (Douglass and Strausfeld, 2003, reviews Borst and Haag, 2002; Borst et al., 2010). LPTCs can be divided into two types: HS cells signal motion in the horizontal direction, whereas VS cells signal motion in the vertical direction (Borst et al., 2010; Joesch et al., 2008).

A

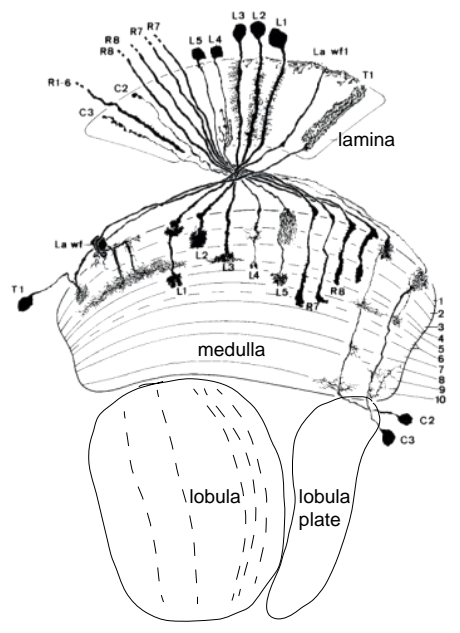

B

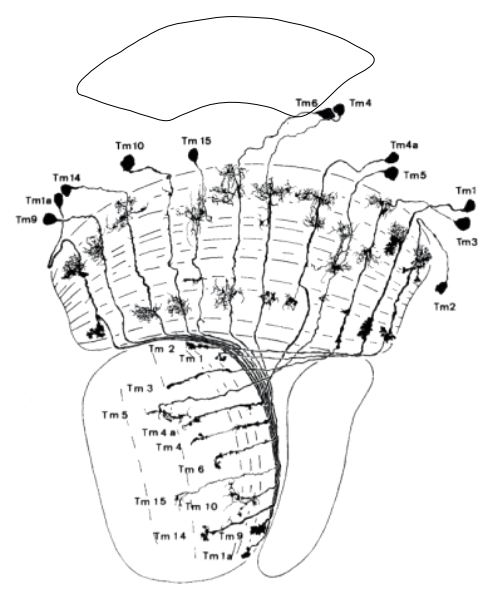

C

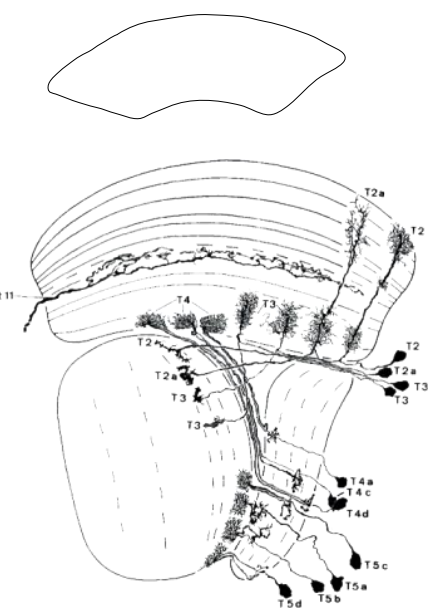

Figure 3: Drosophila visual system schematic depicting ganglia and different cell types. 
Three ganglia are shown. (A) 12 cell types of the lamina are shown. (B) Transmedullary (Tm) cells in the medulla. (C) T4 and T5 come in 4 subtypes a-d. Modified after (Fischbach and Dittrich, 1989).

\subsection{Processing of visual information in Drosophila}

Vision starts with photons emitted from a light source hitting the eye. Photons hitting the eye travel through the transparent cornea and then through the lens, which focuses the light onto the retina. There, the photons are absorbed and transformed into electrical potentials, which are then sent towards the brain.

\subsubsection{Photoreceptors}

R1-R6 photoreceptors in the Drosophila eye house around 30,000 microvilli, which sample incoming photons and each microvillus transduces photons into transient electrical responses or so-called quantum bumps (Figure 4A). Each quantum bump is a result of the opening of 15-20 ion channels (Henderson et al., 2000). An increase in light intensity lead to absorption of more photons and thus an overlap of quantum bumps, which then fuse to build a graded light response (Figure 4B-C) (review Honkanen et al., 2017). Invertebrate photoreceptors depolarize to light, whereas vertebrate rods and cones hyperpolarize. Channels in invertebrate photoreceptors are thus closed in the dark, whereas channels in rods and cones are open in the dark. Invertebrate photoreceptors have an advantage in dim light, because just a few of the channels have to open in order to detect a photon in comparison to vertebrate rods and cones where several hundred channels must close in order to detect a signal above noise (Hardie, 2012; Henderson et al., 2000).

Photoreceptors can compute contrast irrespective of illumination. This is due to visual adaptation, which enables photoreceptors to adapt to mean luminance. Adapting to the mean luminance allows photoreceptors to compute contrast (relative light changes) accurately and without saturating across a wide luminance range (Laughlin, 1989). Voltage recordings from fly photoreceptor cells showed that photoreceptors respond to a prolonged bright light illumination with an initial transient phase, followed by the sustained plateau. When photoreceptors adapt to the background, the plateau response amplitude varies with the intensity, i.e. the amplitude of the plateau increases to the increasing background intensity (Laughlin and Hardie, 1978; Laughlin et al., 1987). The initial transient phase of the photoreceptor response lasts for $100-200 \mathrm{~ms}$ and encodes contrast, while the sustained plateau component lasts for the stimulus duration and encodes luminance (Laughlin and Hardie, 1978). 
R7 and R8 cells have input impedances 3-6 times greater than R1-R6 (Hardie et al., 1981), a result of a much smaller membrane surface area. Intracellularly recorded responses from R7 and R8 are noisier compared to R1-R6 and show much larger quantum bumps. In addition, the luminance-sensitive plateau component of the R7 and R8 response is typically at $80 \%-90 \%$ of the transient peak, whereas the plateau component of R1-6 is typically at 50\% (Hardie, 2011). Therefore, inner photoreceptors are described as less adapting and are thought to depict a high-acuity system.

A

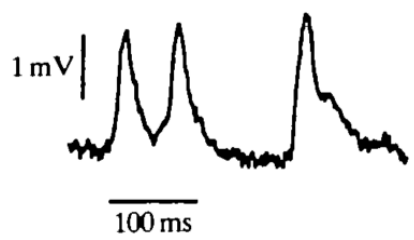

B

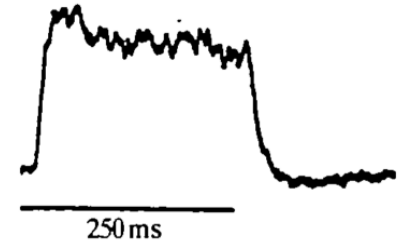

C

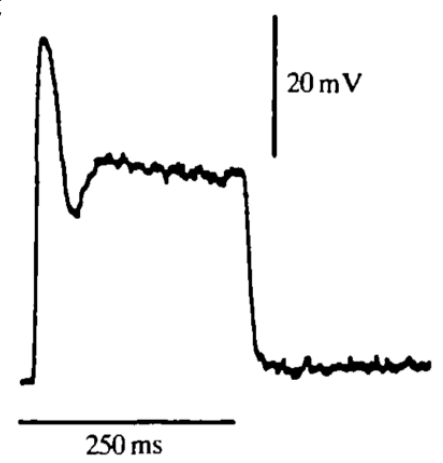

Figure 4: Voltage recordings of photoreceptors to light flashes of different luminances.

(A) Elementary events or quantum bumps, recorded from the photoreceptor cells in response to a sustained dim illumination. (B) Photoreceptor response to $250 \mathrm{~ms}$ long light flashes of intermediate intensities shows the fusion of the quantum bumps and therefore a sustained response. (C) At high intensities, photoreceptors respond to $250 \mathrm{~ms}$ flashes with a transient peak, followed by a steady plateau. Taken from (Laughlin, 1989).

\subsubsection{Lamina Monopolar Cells (LMC)}

Downstream of photoreceptors, LMCs respond in a transient manner. LMC responses can be divided into three parts: 'on' transient, followed by a plateau phase and a transient 'off' response (Figure 5) (Laughlin and Hardie, 1978). The 'on' transient represents an amplification of the photoreceptor input, the plateau phase decays to the previous baseline in 1-5 s and the 'off' transient occurs at the offset of light (Laughlin and Hardie, 1978). Therefore, a major transformation is thought to happen at the photoreceptor synapse with the LMCs: the photoreceptor signal is amplified, sign inverted and transformed into a transient response by discarding information about constant illumination (Laughlin, 1989; Laughlin et al., 1987, Laughlin and Hardie, 1978, Järvilehto and Zettler 1971). This elimination of the luminance information allows LMCs to respond with the same amplitude to the same luminance changes at different light regimes.

However, not all LMCs respond in exactly the same way. It has been described that one type of LMC cells shows responses, which do not return to baseline, but instead show a plateau response throughout the course of the sustained illumination (Hardie and 
Weckström, 1990). There are two known kinds of voltage-gated conductances in LMCs: a delayed rectifier current $\left(\mathrm{K}_{\mathrm{D}}\right)$ and an $\mathrm{A}$ current $\left(\mathrm{K}_{\mathrm{A}}\right)$. $\mathrm{K}_{\mathrm{D}}$ has a slow inactivation whether $\mathrm{K}_{\mathrm{A}}$ has both a faster inactivation and also more rapid activation (Hardie and Weckström, 1990). Intracellular recordings followed by dye fills showed that L3 cells show only $K_{D}$, whereas L1 and L2 neurons show mainly $K_{A}$ conductances. The kinetics of the voltage-gated $\mathrm{K}^{+}$channels is voltage and time dependent and since $\mathrm{K}_{\mathrm{A}}$ and $\mathrm{K}_{\mathrm{D}}$ currents are differentially displayed in different LMCs, this could provide a mechanism for differential frequency-selective transmission of the signal (Rusanen and Weckström, 2016). Another difference between LMCs is that the resting potential of L3 is $-60 \mathrm{mV}$, which is lower than that of both L1 and L2, of which the resting potential is $-40 \mathrm{mV}$. This characteristic allows to separate L3 from other LMCs (Hardie and Weckström, 1990; Rusanen and Weckström, 2016). To sum up, while all LMCs receive the same R1-R6 input and generally thought to amplify contrast-sensitivity inherited from photoreceptors, different types of LMCs characteristic have been described.

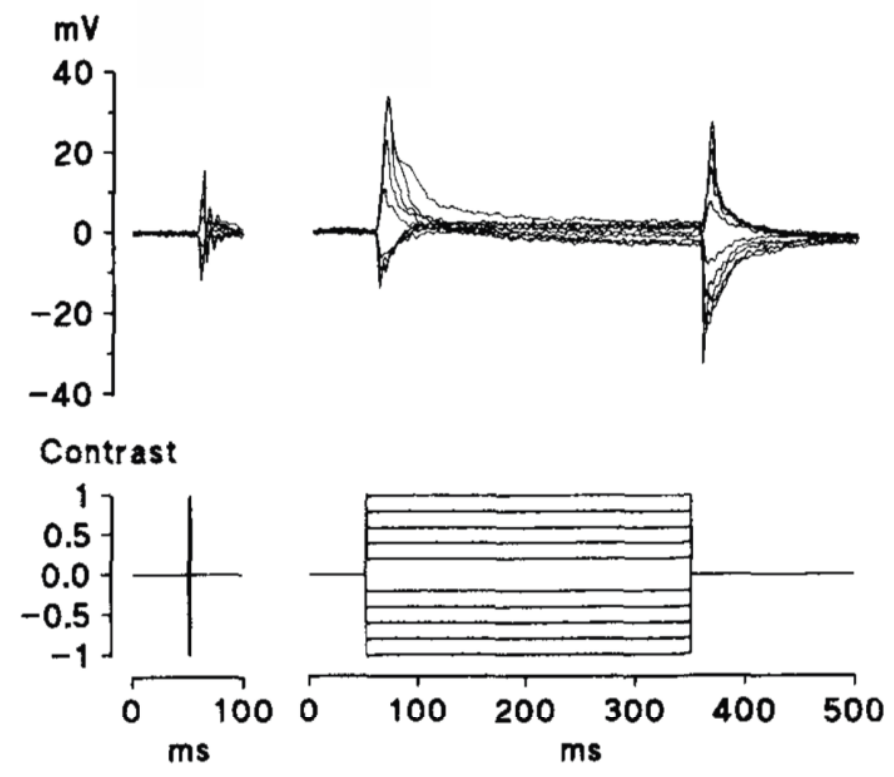

Figure 5. Voltage recordings from LMCs.

Voltage responses recorded from a light adapted LMC in response to $2 \mathrm{~ms}$ (left) and to $300 \mathrm{~ms}$ (right) contrast steps are shown. Taken from (Juusola et al., 1995).

While most of the initial studies generalized across LMC types, the development of cell type specific targeting, the existence of new sensitive $\mathrm{Ca}^{2+}$ indicators, and other genetic tools which allow the manipulation of activity, one could further characterize LMCs and their downstream circuits. Using these tools, researchers identified L1 and L2 as the major inputs to motion detecting circuits, feeding into separate ON or OFF pathways: L1 was described to be the main ON pathway input, while L2 was described to be the major 
input into the OFF pathway (Clark et al., 2011; Joesch et al., 2010; Rister et al., 2007). Thus, the split onto ON and OFF pathways occurs just postsynaptic to photoreceptors. Therefore, it was hypothesized that L1 and L2 could be differentially activated by either positive or negative contrast (Reiff et al., 2010). However, L1 as well as L2 neurons show an increase in calcium level to the light offset and a decrease in calcium to the light onset (Clark et al., 2011). The physiological split into neurons that selectively respond to ON and OFF signals, respectively, was subsequently shown to happen one synapse further downstream, in medulla neurons (Behnia et al., 2014).

Subsequently, the lamina neuron L3, was identified in a forward genetic screen to also contribute to motion detection in Drosophila (Silies et al., 2013; Tuthill et al., 2013). Till this time, researchers thought that L3 is involved in spectral preference and landmark orientation (Gao et al., 2008; Rister et al., 2007). Both L1 and L3 were shown to make important contributions to OFF-edge motion detection (Silies et al., 2013). Silencing L3 led to a deficit in in fly's ability to follow very slowly moving stimuli (Tuthill et al., 2013). As L1 and L2 neurons, L3 neurons decreased to the onset of light and increased to the light offset. In contrast to L1 and L2 responses, which have biphasic temporal filters (Clark et al., 2011), responses of L3 neurons are more sustained and display a monophasic temporal filter (Silies et al., 2013). Therefore, L3 neurons show sustained calcium responses, whereas L1 and L2 neurons respond transiently to the sustained input (Clark et al., 2011; Silies et al., 2013).

As said before (see 2.2.2 Lamina), the lamina houses 12 cell types. The phenotypic effects upon silencing other lamina neurons than L1, L2 and L3 were much sparser. For example, silencing of $\mathrm{C} 2$ and $\mathrm{C} 3$ resulted in reduced behavior to regressive motion stimuli (Tuthill et al., 2013). However, the main result of this study was that only a few lamina output neurons are truly necessary for motion detection.

\subsubsection{Medulla neurons}

The medulla is densely packed with different types of neurons. Anatomical studies identified at least 80 cell types (Fischbach and Dittrich, 1989; Nern et al., 2015). Connectomic studies then identified medulla cells, which make synapses with lamina neurons and feed into the first direction-selective T4 and T5 neurons (Rivera-Alba et al., 2011; Shinomiya et al., 2014). Electrophysiological recordings, in vivo two-photon microscopy as well as behavioral studies showed that the split into ON and OFF pathway is conserved in the medulla and different medulla neurons were assigned to both of the pathways (Figure 6) (Behnia et al., 2014; Fisher et al., 2015a; Serbe et al., 2016; Shinomiya et al., 2014; Strother et al., 2017; Takemura et al., 2013; Yang et al., 2016). 
These medulla neurons exhibit differential spatial and temporal filtering properties and can thus implement the spatiotemporal comparisons required for motion detection. In the ON pathway, L1 neurons provide input mainly to Mi1 and Tm3, whereas in the OFF pathway, L2 provides the main input to Tm1, Tm2 and Tm4 (Takemura et al., 2013). The other OFF pathway input neuron, L3, provides input to Tm9, Mi1, Mi9 and Tm20 (Takemura et al., 2013, 2017). Although Mi9 receives input from the OFF pathway neuron, Mi9 feeds onto the ON pathway(Takemura et al., 2013, 2017). Thus, the ON and the OFF pathways are not as strictly separated as it was initially thought (Fisher et al., 2015a; Takemura et al., 2013). While Mi1 neurons in the ON pathway selectively respond to contrast increments (Behnia et al., 2014) are transient and show band-pass filter characteristics, Mi9 neurons respond with a depolarization to OFF stimuli (Strother et al., 2017) are sustained and show a pure low-pass filter (Arenz et al., 2017). Another medulla neuron, which shows sustained responses to sustained input is Tm9, which receives the main input from L3. Unlike Tm9 and Mi9 sustained responses, Tm1, Tm2 and Tm3 show transient responses to sustained stimuli (Behnia et al., 2014; Meier et al., 2014; Serbe et al., 2016; Yang et al., 2016). None of the medulla neurons are direction-selective, indicating that direction selectivity is computed below (Fisher et al., 2015b).

\subsubsection{Neurons in the lobula complex}

The above mentioned medulla cells make synapses with $\mathrm{T} 4$, which respond to contrast increments and T5 neurons, which respond selectively to contrast decrements. Four different T4/T5 subtypes project to one of the four layers of the lobula plate in a way that adjacent layers represent opposite directions of motion. The preferred direction of layer one is front-to-back, of layer two is back-to-front, of layer three is upwards and of layer four is downwards motion (Maisak et al., 2013). In the recent past, there was a focus on understanding the direction selectivity mechanisms. Directional-selective responses of T4 / T5 cells are a result of a selective amplification of local inputs (Fisher et al., 2015b). T4 / T5 cells are orientation selective, meaning that they respond stronger to the static features positioned orthogonally to their preferred direction of motion. The orientation and direction selectivity together thus sharpens directional tuning (Fisher et al., 2015b). Subsequent studies reported that T5 cells gain their direction selectivity by incorporating both preferred direction (PD) enhancement and null direction (ND) suppression (Haag et al., 2016, 2017; Leong et al., 2016).

$\mathrm{T} 4$ and $\mathrm{T} 5$ cells terminate in the lobula plate layers so that adjacent layers are sensitive to opposite directions of motion (Maisak et al., 2013). There, T4 and T5 synapse onto the 
dendrites of the wide-field motion-sensitive tangential cells (LPTCs). LPTCs are large neurons whose dendritic tree spans the lobula plate. They project in a layer specific way in the lobula plate so that the directional preference of a single LPTC matches with the direction-selectivity of the T4 and T5 axon terminals in that layer (Figure 6). In contrast to sparse electrophysiological data of medulla neurons, the big size and accessibility of LPTCs made these neurons prime targets for electrophysiological studies. Due to the bigger size of the animals, a lot of electrophysiological recordings have been done in blowflies. These studies have shown that LPTC responses are also direction-selective (Hausen, 1976; Hengstenberg et al., 1982). Different studies showed that LPTCs system allows animals to be aware of their own motion (Franz and Krapp, 2000; Krapp and Hengstenberg, 1996) and that the Drosophila behavioral state alters the properties of the LPTCs (Chiappe et al., 2010; Maimon et al., 2010). LPTCs depolarize or hyperpolarize to a moving stimulus in their PD or in the ND, respectively. This is achieved by lobula plate intrinsic cells, which provide inhibitory glutamatergic input to LPTCs (LPi) (Mauss et al., 2015). In brief, T4/T5 cells provide a direct excitatory input onto VS cells in layer 4. Lpi3-4 cells receive input from excitatory T4/T5 cells in layer 3 and send a signal to the adjacent layer onto VS cells via an inhibitory synapse (Mauss et al., 2015). Downstream, HS and VS cells functionally connect with different types of descending neurons (DNs) (Suver et al., 2016). There are estimated to be around 1000 DNs in Drosophila and they connect the central brain with the downstream thoracic ganglion (Hsu and Bhandawat, 2016; Namiki et al., 2018).

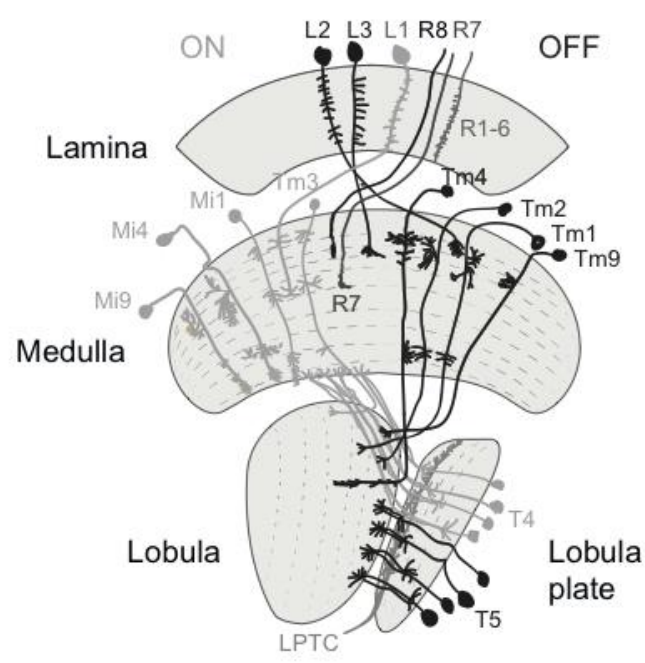

Figure 6. Schematic of the Drosophila visual system.

Two distinct ON (light gray) and OFF (black) pathways, specialized to detect moving contrast increments and decrements. Only cell types of $\mathrm{ON}$ and OFF pathways that are considered to be part of core motion detecting are shown. Neurons depicted are examples of neurons from one column, and repeat $\sim 800$ times in the visual system. 


\subsection{Vision in Changing Light conditions}

In order to orient in an environment, an animal has to reliably discriminate subtle differences in contrast, spatial position and color. When we are moving in an outdoor environment, we encounter big variations in light intensities. The most obvious changes occur between bright daylight and night and an animal must cope with these light changes in order to appropriately navigate through the environment throughout the day. In addition, an animal has to detect fast luminance changes produced by an animal's own movement as it navigates from one location to another. Therefore, survival of many animas depends on detecting contrast in rapidly changing environments.

Below, I summarize the retinal adaptation and the mechanisms that adjust sensitivity, which allow visual systems to perform well in different light conditions.

\subsubsection{Visual adaptation}

Diurnal animals live in natural scenes where the light intensities (photons $\mathrm{s}^{-1} \mu \mathrm{m}^{-2}$ ) sometimes span several orders of magnitude e.g. when comparing areas in the shade with areas exposed to direct sunlight. On the other side, e.g. photoreceptors have a much more limited output range, spanning e.g. 30-60 mV (Warrant and McIntyre, 1992). Thus, visual systems need to somehow map a huge dynamic input from a visual scene into a narrow range of neuronal responses such that the signal is not lost in noise or saturated. Adaptation is a common property of sensory systems. Adaptation can be described as a decrease in a response in time during the constant stimulus presentation. In other words, neurons should transmit a message with the same information content in different situations and to do so, they have to use their output range regardless of different situations (Cover and Thomas, 2006).

\subsubsection{Time course of the adaptation}

Insect photoreceptor cells show two phases of adaptation. A fast phase, lasting for $\sim 100$ $\mathrm{ms}$ and attenuating to a plateau level, and a slow phase, representing the reduction of the plateau and lasting for almost 1 minute (Laughlin and Hardie, 1978). It was shown that in skate retina, the light adaptation is very slow as it takes up to 20 minutes for photoreceptor sensitivity to reach a stable level (Dowling and Ripps, 1970). Fast and slow adaptation were also shown to be present in vertebrates (Adelson, 1982). Similar to invertebrates, fast phase of the adaptation operates within seconds after the onset of illumination, whereas a slow phase of adaptation engaged for more than tens of seconds of prolonged illumination (Adelson, 1982; Calvert et al., 2002). In vertebrates, fast adaptation was observed at all light levels, whereas the slow adaptation was nearly 
absent from responses to dim light (Calvert et al., 2002). The fast adaptation mechanisms are well characterized and involve $\mathrm{Ca}^{2+}$ dependent mechanisms in the phototransduction cascade. On the other side, the slow adaptation mechanisms are yet to be discovered.

\subsubsection{Mechanisms of photoreceptor adaptation}

Photoreceptors have the ability to adapt to their input, which allows them to function optimally at wide variations of illumination (Van Hateren, 1997). The response-intensity curve is a good way to show a dynamic range of a photoreceptor cell (Figure 7). Photoreceptor adaptation mechanisms adjust the sensitivity, which results in a new response-intensity curve for each illumination background (Laughlin, 1981b). Thus, photoreceptors adapt to mean illumination, which allows them to process contrast in different luminances (review Laughlin, 1989). How is this achieved? In dim light, photoreceptors need to catch more light as compared to bright light. To do so, the pigment granules in pigment cells surrounding the photoreceptors move and absorb less light in dim light conditions, and therefore expand the visual angle viewed by the photoreceptor waveguides. This widening leads to a higher photon catch (Stavenga, 1979). Thus, adaptation mechanisms involve alteration of the photoreceptor optics.

However, changes in the optics are not the only mechanisms underlying visual adaptation. The process of photoreceptor adaptation also involves phototransduction. In brief, during phototransduction, photoisomerization of a photopigment activates a biochemical reactions cascade, which results in the opening of ion channels positioned on the photoreceptor membrane creating a light current. Because photoreceptor membrane houses additional voltage-sensitive ion channels, these channels shape light information into a voltage response (Juusola and Hardie, 2001). At this level, light adaptation is a result of three main factors: a) quantum bump compression, b) quantum bump timing and c) membrane dynamics (Juusola and Hardie, 2001).

a) Light adaptation causes a decrease in the quantum bump size and the time course of the bumps becomes briefer with increasing light. Decrease in the bump amplitude most likely represents a smaller amount of channels that contribute to one bump or the likelihood of the channel to open (Juusola and Hardie, 2001).

b) In invertebrate photoreceptors, calcium levels increase during photostimulation and this is a major factor in adaptation (Bader, 1976; Brown and Blinks, 1974; Lisman and Brown, 1975). Negative feedback mediated by $\mathrm{Ca}^{2+}$ causes a gain of transduction reduction, i.e. a lowering in the amplitude of the quantum bump. This $\mathrm{Ca}^{2+}$ negative feedback results in a shift of the intensity response function curve along the luminance 
axis (Figure 7). In Drosophila, during adaptation, $\mathrm{Ca}^{2}$-dependent TRP and TRPL channels inhibition is the main mechanism for gain reduction (review Hardie, 2012).

c) Phototransduction reactions limit the the voltage response speed (Juusola and Hardie, 2001). Adaptation to light causes lowering of the photoreceptor membrane impedance. Drosophila photoreceptors express voltage-sensitive potassium channels and lightsensitive channels which together contribute to the the adaptive membrane dynamics.

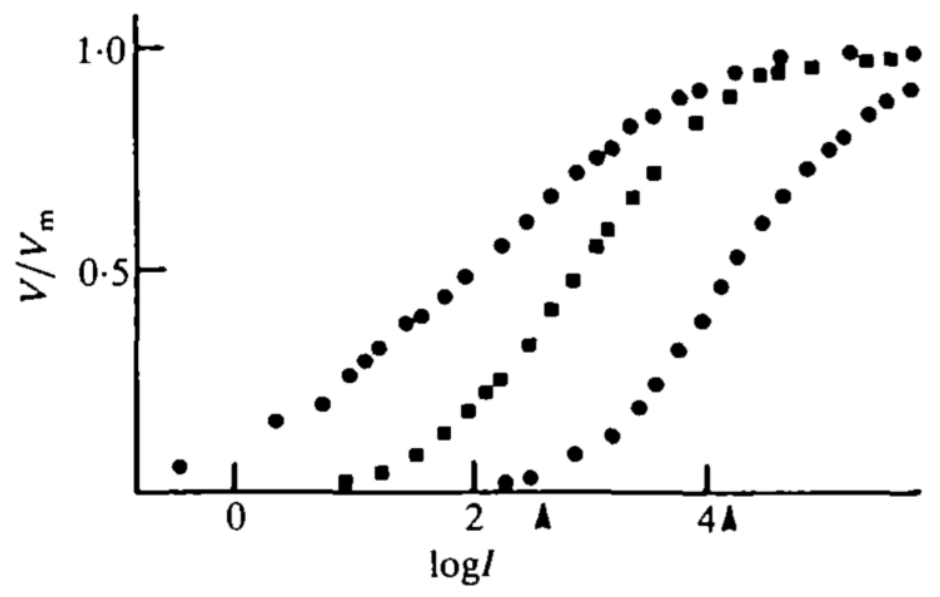

Figure. 7. Adaptation of photoreceptors of the blowfly Calliphora vicina.

Photoreceptor voltage responses. The photoreceptor response amplitude $\mathrm{V}$ was normalized to the maximum response $V_{m}$. When photoreceptors are light adapted, this causes a shift in curves. The left curve was obtained from a dark-adapted cell. The other two curves were obtained from cells, which were adapted to a continuous illumination and are indicated by arrowheads. Taken from (Laughlin, 1989).

\subsubsection{LMC adaptation}

As mentioned above, adaptation ensures that the visual system adjusts its sensitivity to the mean light intensity. Besides photoreceptor adaptation, adaptation also modifies signal transmission at the photoreceptor to LMC synapse (Laughlin, 1989; Laughlin and Hardie, 1978).

Initial experiments suggested that LMCs are unable to light-adapt (Järvilehto and Zettler, 1971). Recordings from both photoreceptors and LMCs then showed that LMCs indeed do light adapt (Laughlin, 1975; Laughlin and Hardie, 1978). It was shown that LMC adaptation differs from photoreceptor adaptation. The majority of LMCs do not show the maintained sustained response to the adapting light as photoreceptors do. In addition, light adaptation causes changes in LMC responses as they become more phasic, or contrast-sensitive, by increasing the amplitude of 'on' and 'off' transients (Figure 8) (Juusola et al., 1995; Laughlin, 1975). Finally, during the adaptation the 'on' transient amplitude decreases, whilst the 'off' transient amplitude increases (Laughlin and Hardie, 
1978). Thus, LMCs adapt to light and this enables them to effectively encode contrast in different luminance regimes.

LMC responses rapidly decay to prolonged illumination, which suggests that the lamina adaptation mechanisms also act quickly (Laughlin and Hardie, 1978). The 'on' and 'off' responses reach the peak amplitude few ms after the change in light intensity (Juusola et al., 1995; Laughlin and Hardie, 1978). It was shown that a dynamic range shift of a LMC of more than one log unit is finished in half a minute, showing that adaptation causes the LMCs sensitivity to be adjusted extremely fast (Laughlin and Hardie, 1978). With brighter intensities, the mechanisms of this fast adaptation are laying on top of slower photoreceptor responses during the beginning of dark adaptation. However, soon after, the slower photoreceptor mechanisms restrict the sensitivity of LMCs (Laughlin and Hardie, 1978). 

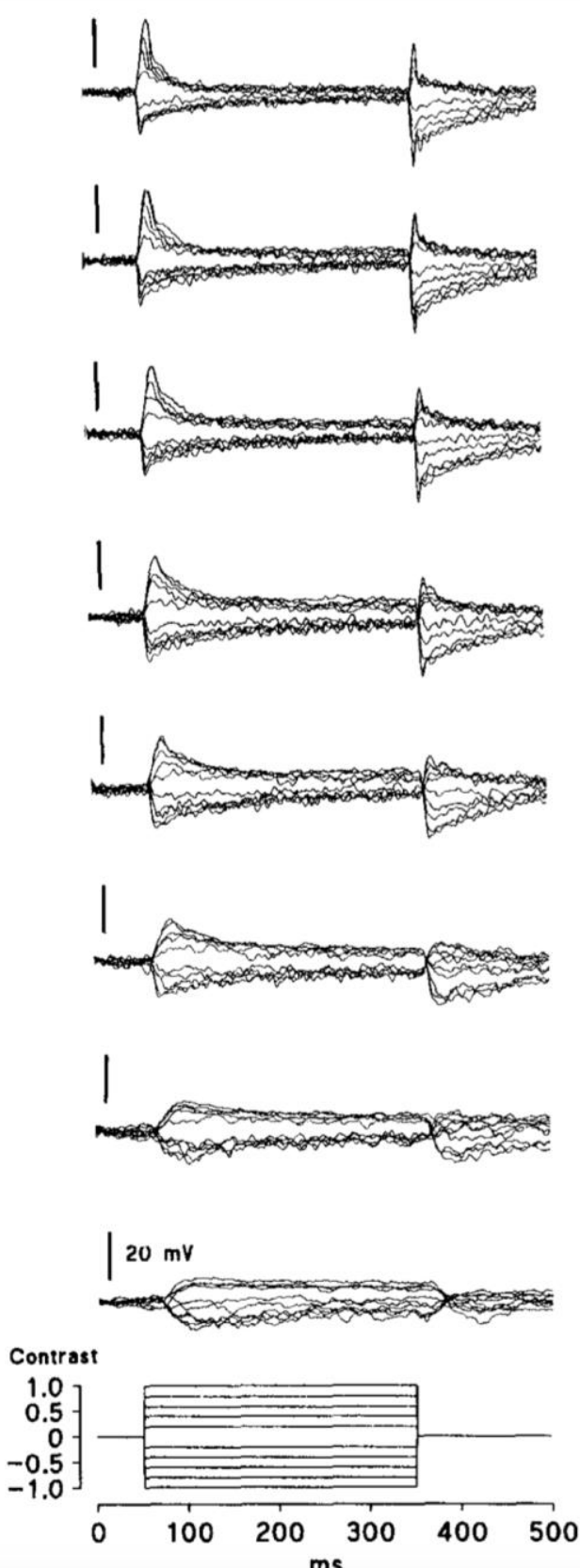

Figure 8. Intracellular responses recorded from LMCs showing the effect of adaptation.

Intracellular voltage recordings of Calliphora vicina LMCs. LMC response examples to 8 different $300 \mathrm{~ms}$ long contrast steps are shown. The responses become more transient with an increasing contrast. Taken from (Juusola et al., 1995).

\subsection{Algorithms of elementary motion detection}

In Drosophila brain, a majority of the neurons are involved in vision, of which one feature is the detection of visual motion. When an animal is navigating in its environment, the visual world moves relative to the animal and global patterns of motion are generated. In addition, moving objects also produce different patterns of 
local motion signals, or changes in luminance over space and time. The nervous system needs to compare inputs from at least two points in time and space so as to assess the direction of motion. Several motion detection computational models have been described and three algorithms have been introduced: Hassenstein-Reichardt Correlator (HRC) (Hassenstein and Reichardt, 1956), the Barlow-Levick Model (BL model) (Barlow and Levick, 1965), and the Motion Energy Model (ME model) (Adelson and Bergen, 1985). The HRC has mostly been used for motion detection in insects, studies of vertebrate retina have favored Barlow-Levick model and the motion energy model has been mainly used in studies of vertebrate visual cortex (review Yang and Clandinin, 2018, Adelson and Bergen, 1985; Barlow and Levick, 1965; Hassenstein and Reichardt, 1956).

In the original simple format, the HRC composes of two subunits, which are mirrorsymmetrically positioned to each other, with each subunit receiving signals from two different points in space (Figure 9). One signal is low-pass filtered and thus delayed with respect to the other and after that, the signals are multiplied. This leads to a differential response to a stimulus moving in different directions. If the stimulus moves in the direction where it first encounters the delayed arm before the non-delayed arm of the subunit, and if the time delay matches the time it takes for the stimulus to hit the nondelayed arm, the signals of these two arms coincide and are multiplied, producing a strong output. Lastly, subtraction of the outputs of both subunits occurs, which results in a fully opponent direction selective signal (Figure 9). If the stimulus is moving in the ND, meaning in the opposite direction (it first encounters the non-delayed arm before the delayed arm) of the subunit, the delay in the circuit produces signals which do not coincide at the multiplication step. This results in small or no output. Therefore, HRC leads to a strong circuit response with positive signals if a stimulus moves in the PD and with no signal if a stimulus moves in the ND (Figure 9).

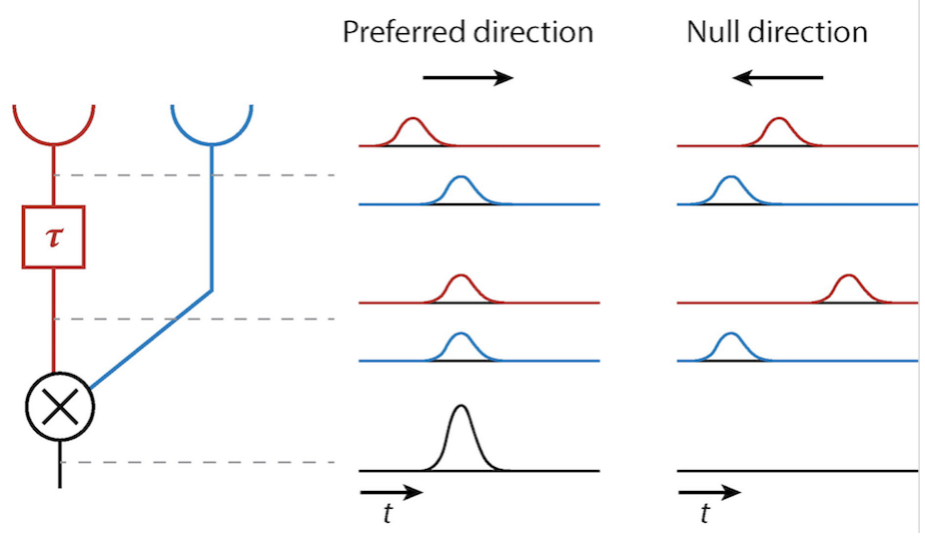

Figure 9. Classical HRC algorithm of elementary motion detection. 
Schematic of the HRC. An edge moving in the PD first encounters the right receptor and then the blue receptor. The red signal is delayed $(\tau)$ and therefore it arrives art the same time as the blue signal at the multiplication step. If the stimulus is moving in the null direction, it produces little to zero response. Taken from Yang and Clandinin, 2018.

While a lot of work has focused on identifying the circuitry implementing an HRC-type computation, it was recently revealed that the biological implementation of motion detection is more complicated than previously thought and that a combination of algorithms is implemented. It has been shown that T4 and T5 neurons can implement both nonlinear amplification of PD and / or non-linear suppression of ND (Fisher et al., 2015, Gruntman et al., 2018, Haag et al., 2016, Leong et al., 2016, Salizar-Gatzimas et al., 2016). In addition, incorporation of a tonic or DC component improves prediction of motion responses (Eichner et al., 2011, Leonhardt et al., 2017). However, it is not yet known how this tonic component is generated and propagated through the circuits.

\subsection{Drosophila tools to manipulate and monitor neuronal activity}

One main goal of systems neuroscience is to comprehend how neuronal circuits function in order to implement computations and to drive an appropriate behavioral response to a sensory input (Clark et al., 2013). Electrophysiology and imaging of neural activity together with pharmacology, as well as genetics, can be used to manipulate and monitor neuronal activities. These techniques make it possible for us to dissect the neural circuit function and to understand the correlations between the activity and behavior (Clark et al., 2013). Recently developed genetic tools allow manipulation and measurement of neuronal activity with cell-specificity (Luo et al., 2018; Venken et al., 2011). The celltype specificity of these tools help us to establish a causal relationship between neurons, circuits and behaviors. A model organism that allows to perform very sophisticated genetic manipulations and the one that we used in our study is Drosophila melanogaster. New tools are continuously being developed, and below I will describe the most frequently used tools, which are currently available for neural circuit dissection in Drosophila.

\subsubsection{Genetic access to Drosophila cell types}

One of the very important and widely used genetic tools developed in Drosophila is the binary expression system Gal4 / UAS (Brand and Perrimon, 1993a). This is a two-part system, which allows us to target gene expression in Drosophila, and therefore allows transcription of a gene to be cell-type or tissue specific. Here, the driver line, expresses 
the Gal4 transcription factor and thus defines a location of expression of an effector gene and the other fly line, the reporter line (UAS), defines which gene should be expressed (Brand and Perrimon, 1993). Crossing a driver line to an effector line produces a progeny with a reporter gene being expressed in a particular cell or tissue (Brand and Perrimon, 1993b; Venken et al., 2011).

Vast Gal4 collections have been generated and are publicly available (Gohl et al., 2011; Jenett et al., 2012). However, many of those lines are not completely cell-type specific as they have a broad expression pattern. This can be problematic when one wants to, for example, image specifically from a certain cell type or when one wants to link certain phenotype to a specific neuron. To solve this problem, additional tools have been developed that make it possible to obtain more specific expression patterns by intersecting the patterns of different Gal4 driver lines (Gohl et al., 2011). For example, one can use a split-Gal4 system where the Gal4 gene coding region is split (Luan et al., 2006; Pfeiffer et al., 2010). Thus, one enhancer drives the expression of the Gal4 activating domain (AD) and a second enhancer drives the DNA-binding domain (DBD) expression. Only an activity of both enhancers in one cell will result in a functional Gal4 protein (Luan et al., 2006; Pfeiffer et al., 2010).

In addition, several other binary systems exist, for example the LexA-lexAop system (Lai and Lee, 2006) and the QF-QUAS system (Potter et al., 2010), which allows expression of different effector types in various cell types simultaneously in a single animal. The collection of LexA and QF systems targeting different cell types is much smaller compared to the Gal4 collection. Thus, the LexA and QF systems are most regularly used in experiments that require several genetic manipulations or the expression of different fluorescence indicators and already use Gal4 system.

Gal4 and QF repressors, Gal80 and QS, respectively, can also be used for restricting gene expression of partially overlapping expression patterns by repressing transcription factors that bind to the $\mathrm{QF}$ or Gal4 $\mathrm{AD}$ and thus prevent the start of transcription (Lee and Luo, 1999; Potter et al., 2010; Riabinina and Potter, 2016).

\subsubsection{Monitoring neuronal activity}

Relatively recent tool developments in Drosophila permit us to directly observe and alter the neuronal activity of neurons of interest. Functional imaging allows us to visualize synaptic input, calcium fluxes, neurotransmitter release and intracellular signaling. Here I will focus on genetically encoded calcium and voltage indicators, which I used in this study to record changes in intracellular calcium and membrane voltage, respectively. 


\subsubsection{Genetically Encoded Calcium Indicators (GECIs)}

Calcium is a universal secondary messenger as it carries messages to practically all important functions of cells and multiple mechanisms tie intracellular $\mathrm{Ca}^{2+}$ concentration to neural activity. Therefore, genetically encoded calcium indicators (GECI) have been developed in order to investigate and visualize neuronal activity associated with calcium transients in the living cells. For example, membrane depolarizations cause synaptic voltage sensitive $\mathrm{Ca}^{2+}$ channels to open, allowing $\mathrm{Ca}^{2+}$ to enter the cell. Thus, $\mathrm{Ca}^{2+}$ concentration is related to membrane voltage and is therefore a good proxy of neuronal activity (Broussard et al., 2014; Göbel and Helmchen, 2007; Riemensperger et al., 2012). The most commonly used GECI is GCaMP. GCaMP is a chimeric protein consisting of a circularly permuted green fluorescent protein (cp-GFP), which is inserted between the calcium binding protein calmodulin (CaM) and an M13 peptide (Figure 10A). Binding of $\mathrm{Ca}^{2+}$ causes CaM-M13 complex conformational changes, which cause a change in fluorescence in the cp-GFP. GCaMP changes its conformation in $\mathrm{a} \mathrm{Ca}^{2+}$ dependent manner, leading to a change in the fluoresce level (Figure 10A). In our study we used GCaMP6f, which has a wide dynamic range, higher $\mathrm{Ca}^{2+}$ binding affinity and the fastest kinetics among the GCaMP family (Chen et al., 2013).

\subsubsection{Genetically Encoded Voltage Indicators}

GECI are commonly used and newer calcium indicators (i.e. GCaMP6f) that are capable of detecting single action potentials (Chen et al., 2013). However, membrane potential as well as subthreshold oscillations change rapidly during an action potential and whereas calcium is a proxy of neuronal activity, its concentration does not directly report membrane potential and thus it can not be used to monitor small changes in voltage that do not result in big calcium fluxes (Koester and Sakmann, 2000; Theis et al., 2016). Therefore, voltage is considered to be the most direct way to monitor neuronal activity, as the electrical potential across the plasma membrane carries key information (Ainsworth et al., 2012). Electrophysiological methods have been successful in monitoring voltage, however, certain experiments are very difficult or impossible to be performed with electrophysiology. Neurons in the Drosophila visual system are tiny, and only very few people succeeded to record from distinct cell types (Behnia et al., 2014; Gruntman and Turner, 2013). However, optical voltage indicators can be of big use, as they allow us to track the voltage signals with higher spatial resolution, from small subcellular regions and from multiple subcellular locations and neighboring neurons (Yang and St-Pierre, 2016). The first voltage reporters were small-molecule dyes (review Chemla and Chavane, 2010; Tsytsarev et al., 2014). The need to label and 
optically record voltage from specific cell types led to the development of genetically encoded voltage indicators (GEVIs). Recently, a genetically encoded voltage sensor, ASAP2, that shows high sensitivity, quick kinetics and compatibility with two-photon microscopy in Drosophila has been developed (Yang et al., 2016). ASAP2f was engineered from ASAP1 (St-Pierre et al., 2014) in which a cp-GFP is introduced into a voltage-sensing domain, VSD (Figure 10B). In contrast to GCaMP, cell depolarization causes a decrease in GFP fluorescence whereas a cell hyperpolarization causes an increase in the fluorescence. ASAP2f showed larger fluorescence changes than ASAP1 and it was stable for more than 10 minutes of imaging (Yang et al., 2016). This study demonstrated that voltage waveforms were conveyed through the lengths of these neurons, whereas calcium responses were compartmentalized, which might indicate differential local signaling. Voltage responses observed with ASAP2f are much smaller in amplitude than calcium responses measured with GCaMP. Therefore, significant averaging over 200-17000 trials was used to achieve the above-mentioned result. While currently available GEVI can already answer previously unanswerable questions, further technological improvements of GEVI are necessary (Yang and St-Pierre, 2016).

A
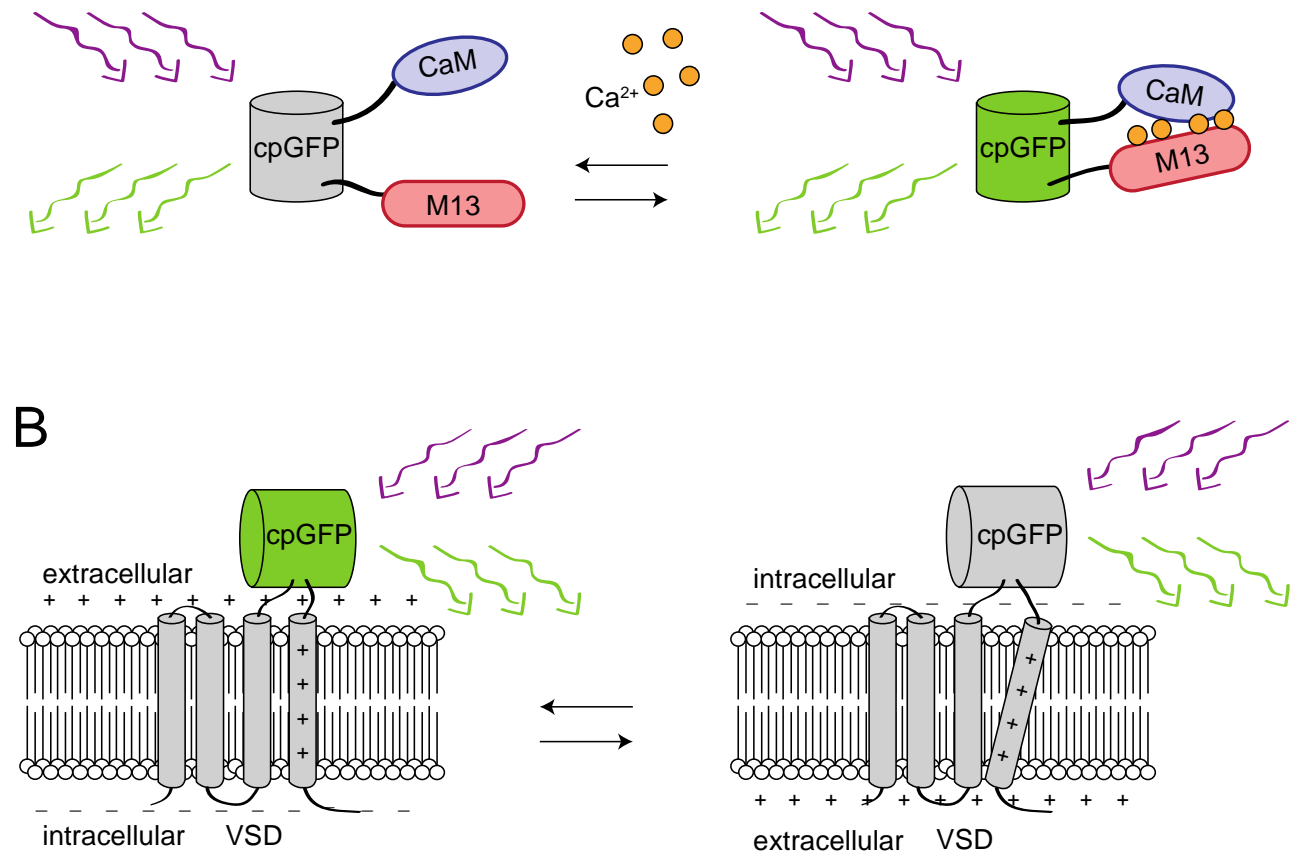

Figure 10. Schematic of a GECI GCaMP6 and GEVI ASAP2f.

(A) Schematic of a GCaMP6 calcium indicator. GCaMP6f is composed of a cp-GFP, which is positioned between CaM and an M13. Calcium binding causes CaM-M13 complex conformational changes, which induces cp-GFP fluorescence changes. GCaMP6 schematic modified after (Broussard et al., 2014). (B) 
Schematic of an ASAP voltage sensor. Changes in membrane potential cause a transmembrane helix of a VSD to move. This causes a change in the cp-GFP fluorescence. ASAP schematic modified after (Yang et al., 2016).

\subsubsection{Genetic manipulation of neural activity}

Drosophila is a great organism to study both the function of genes, as well as the function of neuronal cell types. Below I will summarize currently most commonly used tools for manipulating neuronal activity.

One of the important tools in neuroscience is to inactivate specific neurons. In Drosophila, the two most commonly used techniques for silencing neuronal output are shibire $^{\text {ts }}$ and Kir2.1. shibire encodes for the Drosophila dynamin gene, a GTPase required for the synaptic vesicle endocytosis and recycling (Van Der Bliek and Meyerowrtz, 1991). The mutant shibire allele, shits, is a temperature-sensitive missense mutation in the GTPase protein that acts as a dominant negative allele. Heating the flies to the restrictive temperature $\left(\sim 29^{\circ}\right)$ induces the mutant allele. This reversibly eliminates synaptic transmission in the expressing cell population. Returning the flies to permissive temperatures, allows dynamin to return to the normal function and vesicular recycling continues (Van Der Bliek and Meyerowrtz, 1991). It is worth mentioning that this tool prevents neural communication via chemical synapse, therefore leaving electrical synapses intact (Kitamoto, 2001). When shits is overexpressed in specific neurons (i.e. by using the GAL4/UAS system), this allows spatially and temporally restricted perturbation of the neuronal activities (Kitamoto, 2001).

Another way to silence neurons is by interfering with membrane depolarization. Kir2.1 encodes for a mammalian inward rectifying $\mathrm{K}^{+}$channel which reduces the membrane resting and thus prevents depolarization (Baines et al., 2001). Exogenous Kir2.1 channel expression in Drosophila neurons prevents activation of the neuron and thus silencing these neurons. Unlike shits, Kir2.1 is not restricted in time and therefore chronic manipulations of membrane potential may lead to homeostatic compensation. On the other hand, it is affecting chemical transmission as well as transmission across gap junctions (Baines et al., 2001). Several reviews argue in favor of UAS-Kir2.1 being the most powerful neuronal silencer (Holmes et al., 2007).

\subsubsection{Altering gene expression}

Different neurons express different genes, which e.g. define neuronal morphology, neuronal physiological properties as well as connectivity. One gene can have widespread functions in the brain. Thus, in order to understand the effect of a gene in a certain neuron, it is important to manipulate a gene activity in only a certain cell type. In 
Drosophila, RNA interference (RNAi), FlpStop and MARCM are the most commonly used techniques for cell-type specific gene manipulation (Dietzl et al., 2007; Fisher et al., 2017; Lee and Luo, 1999). Using these techniques, it is now possible to disrupt the function of practically every gene in the fly genome and then study the effect of the gene disruption.

\subsubsection{RNA interference (RNAi)}

A huge discovery in biology has been the discovery of double-stranded RNA molecules, which regulate the expression of genes (review Novina 2004 - the RNAi revolution). RNAi was first discovered in Caenorhabditis elegans and can be used to silence gene expression by using double-stranded RNA molecules (dsRNA) (Fire et al. 1998). In Drosophila, RNAi has been used to prevent the expression of a specific gene in a specific cell or to systematically probe gene function on a genome-wide scale and it has been successfully used in vivo as well as in cultured cells (Boutros et al., 2004; Dietzl et al., 2007). RNA is triggered by dsRNAs that are cleaved by a dsRNA-specific anzyme into small interfering RNAs (21-23 bp). This causes the activation of ribonucleases that cause degradation of dsRNA-complementary endogenous mRNA. Since endogenous mRNA molecules are degraded, translation of the gene to protein is prevented. Collections of transgenic Drosophila lines have been created where each line expresses a unique transgene encoding dsRNA downstream of the UAS-sequence. In combination with the use of GAL4 lines, this leads to tissue or cell-type-specific gene silencing (review Novina and Sharp, 2004). Despite its benefits and its ease of use, RNAi might produce off-target effects and it can produce hypomorphic phenotypes, which do not mirror the complete loss of function (Carthew and Sontheimer, 2009; Khan et al., 2009).

\subsubsection{Mosaic Analysis with a Repressible Cell Marker (MARCM)}

Another approach for a loss of function of a gene is the introduction of a mutation into the endogenous gene locus. However, since genes are often expressed in many cells, this makes the analysis of cell-type specific phenotypes challenging. In such cases, a conditional loss of function can help. In Drosophila, this can be achieved using meiotic recombination, induced by the FLP/FRT recombinase system (Struhl and Basler, 1993). Here, homozygous mutant cell clones can be generated in a heterozygous background for in principle any gene of interest. In a further development of this, the homozygous mutant clone can be dominantly marked, for example by expressing GFP. This technique is called Mosaic Analysis with a Repressible Cell Marker (MARCM). It relies on sophisticated genetics that lead to the loss of Gal80 repression of Gal4 activity in the mutant clones (Lee and Luo, 1999). Major implications of MARCM include lineage 
analysis, tracking neuronal populations and investigating the function of a specific gene in a cell-type specific way (Luo, 2007). Here, the MARCM technique allowed us to analyze the effect of a homozygous mutation in an otherwise phenotypically wild-type organism (Lee and Luo, 2001).

\subsection{Aims of the thesis}

Lamina monopolar cells (LMCs) were shown to possess transient responses and signal contrast while discarding luminance information (Laughlin, 1989). Most studies on LMCs did not distinguish between different LMC types, however, some distinct LMC properties have been reported using intracellular voltage recordings (Uusitalo et al., 1995, Rusanen and Weckström 2016) as well as calcium recordings (Silies et al., 2013). Cell-type specific recordings from two input to OFF pathway, L2 and L3, showed that L3 responses are much more sustained than L2 and display a monophasic linear temporal filter (Silies et al., 2013, Uusitalo et al., 1995). Therefore, we wanted to understand why do we have two fundamentally different OFF pathway inputs.

The three major aims of my dissertation were:

Aim 1:

- Understand what visual features L2 and L3 are sensitive to.

Aim 2:

- Identify the molecular, cellular and circuit mechanisms that shape the distinct physiological properties of L2 and L3.

Aim 3:

- Study the integration of L2 and L3 properties in downstream circuitry?

We used in vivo two-photon microscopy and fly genetics in order to link the molecular specialization and circuit interactions that shape physiological properties of identified cell types. 


\section{MATERIAL AND METHODS}

\subsection{Fly husbandry and strains}

Flies used for 2-photon imaging experiments were raised on a standard molasses-based food at $25^{\circ} \mathrm{C}$. The incubator with flies was set on a $12: 12$ hour light-dark cycle at $55 \%$ humidity. Imaging experiments were conducted at room temperature $\left(20^{\circ} \mathrm{C}\right)$. Female flies of all genotypes were imaged within 2-3 days after eclosion.

\subsubsection{Drosophila strains used in this study}

Drosophila strains used for in vivo two-photon imaging are provided in Table 1 and in Table 2.

Table 1. Fly genotypes used in this study.

\begin{tabular}{|c|c|c|}
\hline Name & Genotype & Figure \\
\hline \multicolumn{3}{|l|}{ Imaging } \\
\hline L3>>GCaMP6f & w+; L3[MH56]-Gal4 / +; UAS-GCaMP6f /+ & $\begin{array}{l}\text { Fig. } 15,16,17 \text {, } \\
19,20,21,24, \\
25,35,44\end{array}$ \\
\hline L2>>GCaMP6f & w+; UAS-GCaMP6f / +; L2[21Dhh]-Gal4 / + & $\begin{array}{l}\text { Fig. 16, 17, 18, } \\
20,21,23,25 \text {, } \\
30,44\end{array}$ \\
\hline$L 3>>A S A P 2 f$ & w+; L3[MH56]-Gal4 / +; UAS-ASAP2f / + & Fig. 22 \\
\hline$L 2>>A S A P 2 f$ & w+; UAS-ASAP2f +; L2[21Dhh]-Gal4 / + & Fig. 22 \\
\hline L3 ort rescue & $\begin{array}{l}\text { w+; UAS-ort / UAS-GCaMP6f; L3[0595]-Gal4, } \\
\text { ort[1],ninaE[1] / Df(3R)BSC809 }\end{array}$ & Fig. 36 \\
\hline L2 ort rescue & $\begin{array}{l}\text { w+; UAS-ort / UAS-GCaMP6f; L2[21Dhh]-Gal4, } \\
\text { ort[1],ninaE[1] / Df(3R)BSC809 }\end{array}$ & Fig. 32, 33 \\
\hline L3 ort -/- & $\begin{array}{l}\text { w+; + / UAS-GCaMP6f; L3[0595]-Gal4, } \\
\text { ort[1],ninaE[1] / DfBSC809 }\end{array}$ & Fig. 36 \\
\hline L2 ort -/- & $\begin{array}{l}\text { w+; +/ UAS-GCaMP6f; L2[21Dhh]-Gal4, } \\
\text { ort[1],ninaE[1] / Df(3R)BSC809 }\end{array}$ & Fig. 32, 33 \\
\hline L3 ort +/- & $\begin{array}{l}\text { w+; UAS-ort / UAS-GCaMP6f; L3[0595]-Gal4, } \\
\text { ort[1],ninaE[1] / + }\end{array}$ & Fig. 26, 36 \\
\hline L2 ort +/- & $\begin{array}{l}\text { w+; UAS-ort / UAS-GCaMP6f; L2[21Dhh]-Gal4, } \\
\text { ort[1],ninaE[1] / + }\end{array}$ & Fig. 26, 32, 33 \\
\hline $\begin{array}{l}\text { L2 ort } \\
\text { overexpression }\end{array}$ & w+; UAS-GCaMP6f / UAS-ort; L2[21Dhh]-Gal4 / + & Fig. 34 \\
\hline $\begin{array}{l}\text { ort-/-,ninaE-/- } \\
\text { L3>>ort rescue }\end{array}$ & $\begin{array}{l}\text { w+; UAS-ort / UAS-GCaMP6f; L3[0595]-Gal4, ort[1], } \\
\text { ninaE[1] / ort[1], ninaE[1] }\end{array}$ & Fig. 26 \\
\hline ort-/-,ninaE-/- & W+; UAS-ort / UAS-GCaMP6f; L2[21Dhh]-Gal4, & Fig. 26 \\
\hline
\end{tabular}




\begin{tabular}{|c|c|c|}
\hline L2>>ort rescue & $\operatorname{ort}[1], \operatorname{ninaE}[1] / \operatorname{ort}[1], \operatorname{ninaE}[1]$ & \\
\hline $\begin{array}{l}\text { ort-/-, ninaE-/- L3- } \\
\text { Gal4 control }\end{array}$ & $\begin{array}{l}\text { w+; + /UAS-GCaMP6f; L3[0595]-Gal4, ort[1], } \\
\text { ninaE[1] / ort[1], ninaE[1] }\end{array}$ & Fig. 26 \\
\hline $\begin{array}{l}\text { ort-/-, ninaE-/- L2- } \\
\text { Gal4 control }\end{array}$ & $\begin{array}{l}\text { w+; + /UAS-GCaMP6f; L2[21Dhh]-Gal4, ort[1], } \\
\text { ninaE[1] / ort[1], ninaE[1] }\end{array}$ & Fig. 26 \\
\hline $\begin{array}{l}\text { rh1-norpA rescue, } \\
\text { imaging L3 }\end{array}$ & $\begin{array}{l}\text { norpA[36]; rh1-norpA / QUAS-GCaMP6f; / L3[0221]- } \\
Q F /+\end{array}$ & Fig. 28 \\
\hline $\begin{array}{l}\text { panR8-NorpA } \\
\text { rescue, } \\
\text { imaging L3 }\end{array}$ & $\begin{array}{l}\text { norpA[36]; panR8-norpA / QUAS-GCaMP6f; / } \\
\text { L3[0221]-QF / + }\end{array}$ & Fig. 28 \\
\hline $\begin{array}{l}\text { rh1-norpA control, } \\
\text { imaging L3 }\end{array}$ & w+; rh1-norpA / QUAS-GCaMP6f; / L3[0221]-QF / + & Fig. 28 \\
\hline $\begin{array}{l}\text { panR8-NorpA } \\
\text { control, } \\
\text { imaging L3 }\end{array}$ & $\begin{array}{l}\text { w+; panR8-norpA / QUAS-GCaMP6f; / L3[0221]-QF / } \\
+\end{array}$ & Fig. 28 \\
\hline $\begin{array}{l}\text { rh1-NorpA rescue, } \\
\text { imaging } L 2\end{array}$ & $\begin{array}{l}\text { norpA[36]; rh1-norpA /lexAop-GCaMP6f; } \\
\text { L2[19D12]-LexA /+ }\end{array}$ & Fig. 27 \\
\hline $\begin{array}{l}\text { panR8-NorpA } \\
\text { rescue, } \\
\text { imaging L2 }\end{array}$ & $\begin{array}{l}\text { norpA[36]; panR8-norpA / lexAop-GCaMP6f; } \\
\text { L2[19D12]-LexA /+ }\end{array}$ & Fig. 27 \\
\hline $\begin{array}{l}\text { rh1-NorpA control, } \\
\text { imaging L2 }\end{array}$ & $\begin{array}{l}\text { w+; rh1-norpA /lexAop-GCaMP6f; L2[19D12]-LexA } \\
/+\end{array}$ & Fig. 27 \\
\hline $\begin{array}{l}\text { panR8-NorpA } \\
\text { control, } \\
\text { imaging L2 }\end{array}$ & $\begin{array}{l}\text { w+; panR8-norpA /lexAop-GCaMP6f; L2[19D12]- } \\
\text { LexA / + }\end{array}$ & Fig. 27 \\
\hline ninaE-GCaMP6f & $w+;$ ninaE-GCaMP6f / +; + /+ & Fig. 29 \\
\hline L3 MARCM control & $\begin{array}{l}\text { w+; FRT40, lexAop-GCaMP6fp10 / tub-Gal80, FRT40, } \\
\text { 27G05-Flp; 9-9-Gal4, UAS-R / BRP-RSRT-STOP- } \\
\text { RSRT-smGFPV5-2A-LexA }\end{array}$ & Fig. 39 \\
\hline L3 MARCM dFezf1 & $\begin{array}{l}\text { w+; dFezf[1] FRT40, lexAop-GCaMP6fp10 / tub- } \\
\text { Gal80, FRT40, 27G05-Flp; 9-9-Gal4,UAS-R / BRP- } \\
\text { RSRT-STOP-RSRT-smGFPV5-2A-LexA }\end{array}$ & Fig. 39 \\
\hline$L 2>>d F e z f$ & $\begin{array}{l}\text { w+; lexAop-GCaMP6fp10attp5 / L2[16HO3]- } \\
\text { LexA,lexAop-myrtdTOM; 11-164-Gal4 / UAS- } \\
\text { dFezf::HA }\end{array}$ & Fig. 37 \\
\hline $\begin{array}{l}\text { L2>>dFezf, } \\
\text { Gal4 control }\end{array}$ & $\begin{array}{l}\text { w+; lexAop-GCaMP6fp10attp5 / L2[16HO3]-LexA, } \\
\text { lexAop-myrtdTOM; 11-164-Gal4 /+ }\end{array}$ & Fig. 37 \\
\hline $\begin{array}{l}\text { L2>>dFezf, } \\
\text { UAS-control }\end{array}$ & $\begin{array}{l}\text { w+; lexAop-GCaMP6fp10attp5 / L2[16HO3]-LexA, } \\
\text { lexAop-myrtdTOM; + /UAS-dFezf::HA }\end{array}$ & Fig. 37 \\
\hline L2 imaging & $\begin{array}{l}\text { w+; lexAop-GCaMP6fp10attp5 / L2[16HO3]-LexA, } \\
\text { lexAop-myrtdTOM; +/+ }\end{array}$ & Fig. 37 \\
\hline L3 dFezfRNAi & $\begin{array}{l}\text { w+; UAS-GCaMP6f / L3[MH56]-Gal4; UAS-shi[ts], } \\
\text { UAS-dFezfRNAi / + }\end{array}$ & Fig. 40 \\
\hline L3>>shibire ${ }^{t s}$ & w+; L3[MH56]-Gal4 / +; UAS-GCaMP6f / UAS-shi[ts] & Fig. 41 \\
\hline L3 HisCl1 RNAi & $\begin{array}{l}\text { w+; L3[MH56]-Gal4 / TriP.HMJ21280; UAS-GCaMP6f } \\
/+\end{array}$ & Fig. 43 \\
\hline
\end{tabular}




\begin{tabular}{|c|c|c|}
\hline L3 Ace $e^{R N A i}$ & $\begin{array}{l}\text { w+; L3[MH56]-Gal4 / +; UAS-GCaMP6f / } \\
\text { TriP.HMJ21280 }\end{array}$ & Fig. 43 \\
\hline L3 Shawl RNAi & $\begin{array}{l}\text { w+; L3[MH56]-Gal4 / +; UAS-GCaMP6f / } \\
\text { TriP.JF01837 }\end{array}$ & Fig. 43 \\
\hline$L 3 b t s z^{R N A i}$ & $\begin{array}{l}\text { w+; L3[MH56]-Gal4 / TriP.HMS02146; UAS- } \\
\text { GcaMP6f / + }\end{array}$ & Fig. 43 \\
\hline$L 2 I h^{R N A i}$ & $\begin{array}{l}\text { w+; UAS-GCaMP6f / +; L2[21Dhh]-Gal4 / } \\
\text { TriP.HMC03319 }\end{array}$ & Fig. 43 \\
\hline$L 2 S l o b^{R N A i}$ & $\begin{array}{l}\text { w+; UAS-GCaMP6f / +; L2[21Dhh]-Gal4 / } \\
\text { TriP.HMC04152 }\end{array}$ & Fig. 43 \\
\hline Tm9>>GcaMP6f & w+; Tm9[R24C08]-LexA / lexAop-GCaMP6f ; + /+ & Fig. 44 \\
\hline $\begin{array}{l}\text { Tm9>>GcaMP6f, } \\
\text { L1>>shits }\end{array}$ & $\begin{array}{l}\text { w+; Tm9[R24C08]-LexA, lexAop-GCaMP6f/ } \\
\text { L1[c202a]-Gal4; UAS-shits /+ }\end{array}$ & Fig. 45 \\
\hline $\begin{array}{l}\text { Tm9>>GcaMP6f, } \\
\text { L2>>shits }\end{array}$ & $\begin{array}{l}\text { w+; Tm9[R24C08]-LexA, lexAop-GCaMP6f / +; } \\
\text { L2[21Dhh]-Gal4 / UAS-shits }\end{array}$ & Fig. 45 \\
\hline $\begin{array}{l}\text { Tm9>>GcaMP6f, } \\
\text { L3>>shits }\end{array}$ & $\begin{array}{l}\text { w+; Tm9[R24C08]-LexA, lexAop-GCaMP6f / +; } \\
\text { L3[0595]-Gal4 / UAS-shits }\end{array}$ & Fig. 45 \\
\hline $\begin{array}{l}\text { Tm9>>GcaMP6f, } \\
\text { L1, L3 }>>\text { shits }\end{array}$ & $\begin{array}{l}\text { w+; Tm9[R24C08]-LexA, lexAop-GCaMP6f / } \\
\text { L1[c202a]-Gal4; L3[0595]-Gal4,UAS-shits / + }\end{array}$ & Fig. 45 \\
\hline $\begin{array}{l}\text { Tm9>>GcaMP6f, } \\
\text { L2, L3 }>>\text { shits }\end{array}$ & $\begin{array}{l}\text { w+; Tm9[R24C08]-LexA, lexAop-GCaMP6f / +; } \\
\text { L3[0595]-Gal4, UAS-shits / L2[21Dhh]-Gal4 }\end{array}$ & Fig. 45 \\
\hline
\end{tabular}

Table 2. Key resources of fly lines used.

\begin{tabular}{|l|l|l|}
\hline Experimental Models: Drosophila melanogaster & N/A \\
\hline L3[MH56]-Gal4 & Timofeev et al., 2012 & N/A \\
\hline L2[21Dhh]-Gal4 & Rister et al., 2007 & N/A \\
\hline UAS-ASAP2f & Yang et al., 2016 & N/A \\
\hline ort[1] & Gengs et al., 2002 & \#27380 \\
\hline UAS-ort & Rister et al., 2007 & \#42747 \\
\hline UAS-GCaMP6f & Bloomington Drosophila & N/A \\
\hline L3[0595]-Gal4 & Silies et al., 2013 & \#27380 \\
\hline Df(3R)BSC809 & BDSC & N/A \\
\hline ninaE-GCaMP6f & Asteriti et al., 2017 & N/A \\
\hline $9-9-G a l 4$ & Nern et al., 2008 & N/A \\
\hline $16 H O 3-L e x A$ & Nern et al., 2008 & \\
\hline $11-164-G a l 4 ~$ & Nern et al., 2008 & \\
\hline
\end{tabular}




\begin{tabular}{|c|c|c|}
\hline \{lexAop-myr::tdTomato $\}$ su(Hw)attP5 & Chen et al., 2014 & $\mathrm{~N} / \mathrm{A}$ \\
\hline $27 G 05-F L P(X)$ & Janelia Research Campus & $\mathrm{N} / \mathrm{A}$ \\
\hline lexAop-GCaMP6f & BDSC & $\# 44277$ \\
\hline 79C23S-RSRT-STOP-RSRTsmFPV5-2A-LexA & Peng et al., 2018 & $\mathrm{~N} / \mathrm{A}$ \\
\hline$d F e z f[1]$ & Weng et al., 2010 & $\mathrm{~N} / \mathrm{A}$ \\
\hline UAS-dFezf1-3xHA & Weng et al., 2010 & $\mathrm{~N} / \mathrm{A}$ \\
\hline$U A S$-shi[ts] & BDSC & \#7068 \\
\hline norpA[36] & Bloomquist et al., 1988 & $\mathrm{~N} / \mathrm{A}$ \\
\hline rh1-norpA & $\begin{array}{l}\text { Transgene from Russel } \\
\text { Shortridge }\end{array}$ & $\mathrm{N} / \mathrm{A}$ \\
\hline panR8-norpA & From Mathias Wernet & $\mathrm{N} / \mathrm{A}$ \\
\hline $\begin{array}{l}\text { HisCl1 }{ }^{\text {RNAi }} \\
\text { P\{TriP.HMJ21280\}attP40 }\end{array}$ & BDSC & \#53932 \\
\hline $\begin{array}{l}\text { Ace RNAi } \\
\text { P\{TriP.JF01978\}attP2 }\end{array}$ & BDSC & \#25958 \\
\hline $\begin{array}{l}\text { Shawl } \\
P\{A i \\
P\{\text { TriP. JF01837\}attP2 }\end{array}$ & BDSC & \#25819 \\
\hline $\begin{array}{l}\text { btsz }{ }^{R N A i} \\
P\{\text { TriP.HMS02146\}attP40 }\end{array}$ & BDSC & \#40898 \\
\hline $\begin{array}{l}\text { Ih } \\
\text { P\{TriP.HMC03319\}attP2 }\end{array}$ & BDSC & \#51765 \\
\hline $\begin{array}{l}\text { Slob }{ }^{R A A} \\
\text { P\{TriP.HMC04152\}attP2 }\end{array}$ & BDSC & $\# 55879$ \\
\hline$L 3[0221]-Q F$ & Silies Lab, unpublished & $\mathrm{N} / \mathrm{A}$ \\
\hline 19D12-LexA & BDSC & $\# 52545$ \\
\hline Tm9-LexA & Silies et al., 2015 & $\mathrm{~N} / \mathrm{A}$ \\
\hline L1[c202a]-Gal4 & Rister et al., 2007 & $\mathrm{~N} / \mathrm{A}$ \\
\hline
\end{tabular}

In order to analyze the effect of an L3-specific dFezf homozygous mutation in an phenotypically wild-type organism, we performed the MARCM technique (Lee and Luo, 2001). By mitotic recombination via the FLP/FRT system, we produced homozygous mutant clones (Ashburner, 1989, Golic and Lindquist, 1989). To induce mitotic recombination and generate homozygous L3 neurons for either the FRT40 carrying control chromosome or homozygous for $d F_{e z} f^{1}$, Flp recombinase was expressed using the 27G05-FLP in lamina neuron precursor cells. Since these homozygous clones were no longer expressing tub-Gal80, UAS-controlled R recombinase was expressed in L3 clones using the 9-9 Gal4 (L3 driver line), and induced recombination within the Brp locus, which resulted in the incorporation of smGFP_V5. This also resulted in LexA 
translation, activating the expression of lexAop-GCaMP6f. This allowed us to dominantly label control and $d F e z f^{1}$ mutant clones with GCaMP6f expression, allowing us to record visual response properties.

\subsubsection{Fly food}

Flies used for in vivo two-photon imaging were raised on a standard molasses-based food.

\subsubsection{Fly Food recipe}

- 2 L water

- $16 \mathrm{~g}$ agar

- 120 g cornmeal

-67 g nutritional yeast

- $100 \mathrm{ml}$ corn syrup

- $100 \mathrm{ml}$ molasses

$1 \mathrm{~L}$ of water was put on the hot plate and agar was added while stirring to dissolve the agar and prevent from burning. In another large pot, cornmeal, nutritional yeast, corn syrup and molasses were mixed together. Hot water was gradually added while stirring in order to break up any lumps. The cornmeal mixture was then put into a pot with agar. The mixture was then cooked under a constant stir on hot plate until thickened. This was then autoclaved for 15 minutes and then stirred to allow steam to escape. After some cooling time, $25-20 \mathrm{ml}$ of propionic acid was added. After mixing, the fly food was poured into vials.

\subsection{In vivo two-photon imaging}

\subsubsection{Fly preparation}

For calcium experiments, 2 to 3 days old female flies were used. Flies were hold on ice until anesthetized, placed on the microscope holder and then glued with a UV-sensitive glue (Bondic) onto a sheet of stainless steel foil containing a hole just large enough to fit the fly head and thorax (Figure 11A-F). Mounting and dissection were done at room temperature. Flies were positioned such that their heads were tilted downwards. Thus, only the back of the head was exposed above while the rest of the head was underneath the foil (Figure 11A-D). After mounting, the cuticle on the back of the head was removed using breakable razor blades and fine forceps (Figure 11F). Flies were then positioned under the microscope in a way that they were looking towards the screen (Figure $11 \mathrm{G}, \mathrm{H})$. The flies were perfused with a carboxygenated saline-sugar imaging solution. 
The saline composition was: $103 \mathrm{mM} \mathrm{NaCl}, 3 \mathrm{mM} \mathrm{KCl}, 5 \mathrm{mM}$ TES, $1 \mathrm{mM} \mathrm{NaH}{ }^{2} \mathrm{PO}^{4}, 4 \mathrm{mM}$ $\mathrm{MgCl}^{2}, 1.5 \mathrm{mM} \mathrm{CaCl}^{2}, 10 \mathrm{mM}$ trehalose, $10 \mathrm{mM}$ glucose, $7 \mathrm{mM}$ sucrose, and $26 \mathrm{mM}$ $\mathrm{NaHCO}^{3}$ (see Used solutions for more details). The saline was bubbled with $95 \% \mathrm{O}_{2} / 5 \%$ $\mathrm{CO}_{2}$ and $\mathrm{pH}$ of the bubbled saline was 7.3.

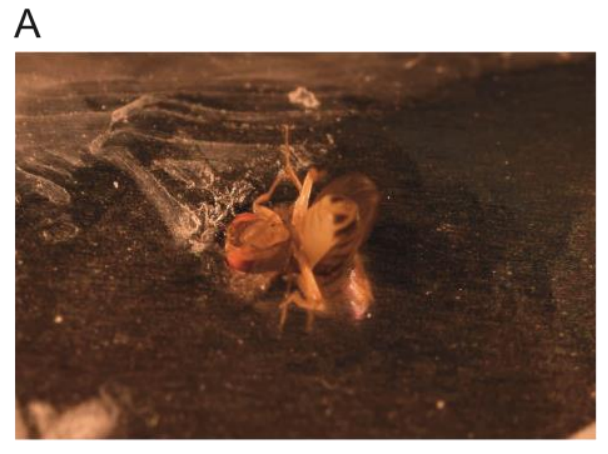

C

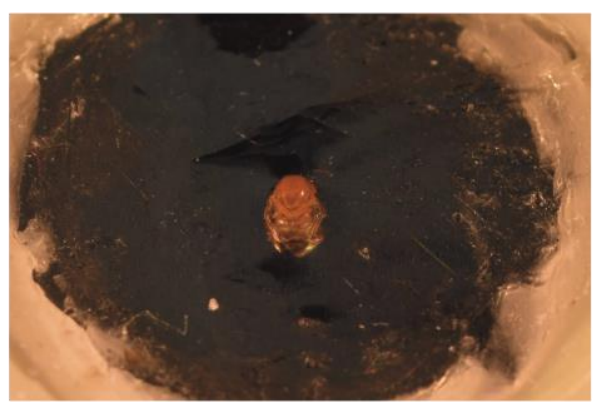

$\mathrm{E}$

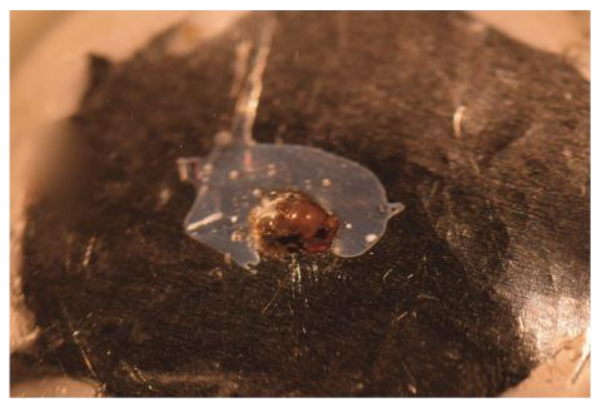

G

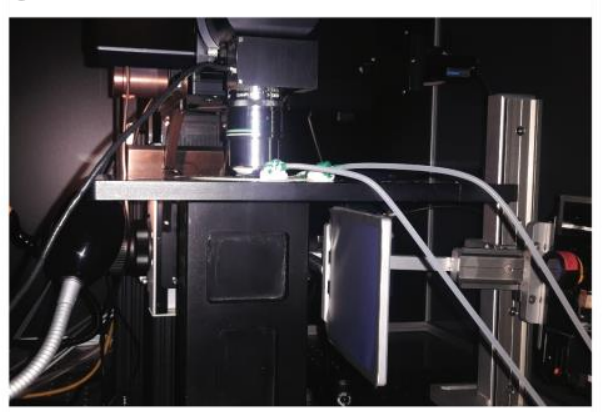

B

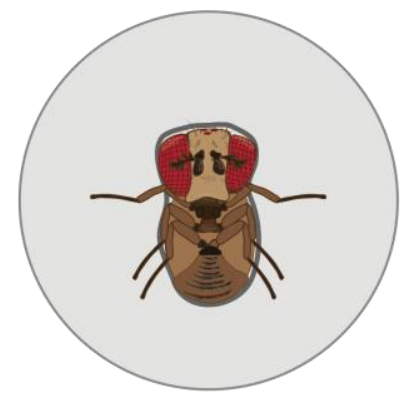

D

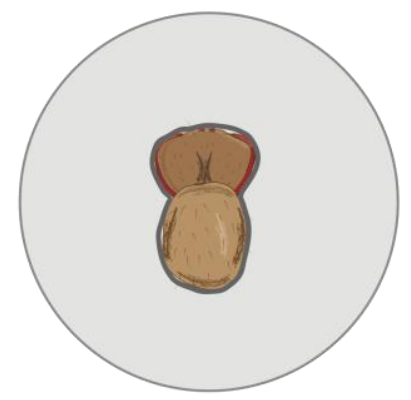

$\mathrm{F}$

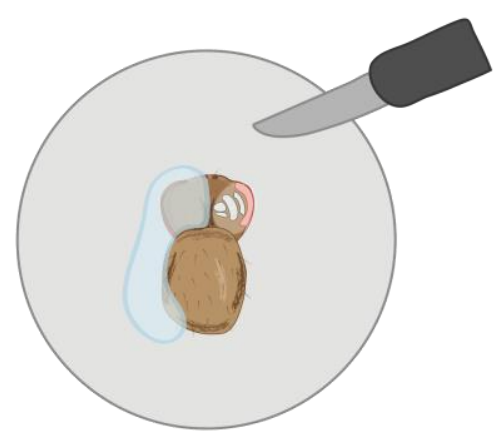

$\mathrm{H}$

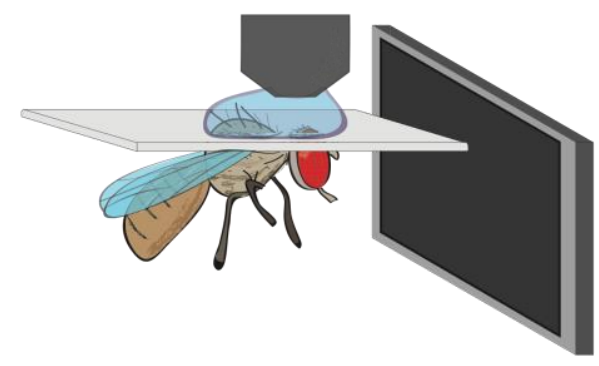




\section{Figure 11. Fly mounting steps for in vivo two-photon imaging}

(A-B) A picture and a schematic showing the first step of the fly mounting. Cold anesthetized flies were first put on the holder with their back on the foil. Then, their thorax was pushed into the hole in the foil. Thus, only the thorax was exposed above the foil and the rest of the fly was below the foil. (C-D) Flies were then repositioned in a way that the back of the head was above the foil, allowing us to perform the dissection. (EF) Flies were glued with a UV-sensitive glue (Bondic). This was followed by an application of the dissection solution. Then the cuticle on the back of the head was removed using breakable razor blades and fine forceps. (G-H) After the dissection the flies were positioned under the microscope in a way that they were looking at the screen.

\subsubsection{Imaging solutions}

\subsubsection{Stock solutions}

$\underline{20 \times \text { Fly saline stock solution (total volume } 500 \mathrm{ml} \text { ): }}$

$-60.19 \mathrm{~g} \mathrm{NaCl}$

$-2.24 \mathrm{~g} \mathrm{KCl}$

$-11.46 \mathrm{~g}$ TES

$-1.20 \mathrm{~g} \mathrm{NaH}_{2} \mathrm{PO}_{4}$

- $8.13 \mathrm{~g} \mathrm{MgCl}_{2} * 6 \mathrm{H}_{2} \mathrm{O}$

- $3.29 \mathrm{~g} \mathrm{CaCl}_{2} * 6 \mathrm{H}_{2} \mathrm{O}$ (only when used for the imaging solution)

- Milli-Q water until 500mL

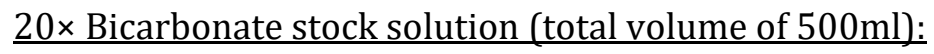

- $21.84 \mathrm{~g} \mathrm{NaHCO}_{3}$

- Milli-Q water until 500mL

Filter both solutions.

\subsubsection{Experimental solutions}

Dissection solution

- $25 \mathrm{ml}$ Fly saline stock solution (without $\mathrm{CaCl}_{2}$ )

- $25 \mathrm{ml}$ Bicarbonate stock solution

- Milli-Q water until 500mL

Imaging solution

- 25 ml Fly saline stock solution (with $\mathrm{CaCl}_{2}$ ) 
- $25 \mathrm{ml}$ Bicarbonate stock solution

- 1.89 g Trehalose

- 0.90 g Glucose

- $1.71 \mathrm{~g}$ Sucrose

- Milli-Q water until 500mL

\subsubsection{Two-photon imaging}

Imaging experiments were performed on a Bruker Investigator 2-photon microscope (Bruker, Madison, WI, USA), equipped with a 25x/NA 1.1 objective (Nikon, Minato, Japan). In order to excite GCaMP6f or ASAP2f, the excitation laser (Spectraphysics Insight DS+) was tuned to $920 \mathrm{~nm}$. When imaging, power of 5-15 $\mathrm{mW}$ was applied at the sample. Emitted light was sent through a SP680 short-pass filter, a 560 lpxr dichroic filter and a 525/70 emission filter and was then detected by photomultipliers (GaAsP detectors, Figure 12). Data was acquired using PrairieView software at a $\sim 20 \mathrm{~Hz}$ frame rate and a frame size $\sim 60 \times 200$ pixels and $8 \mathrm{x}$ optical zoom. An exception were ASAP2f recordings, where a frame rate of $\sim 60 \mathrm{~Hz}$ was used. Axon terminals of Tm9 neurons were imaged in the first layer of the lobula whereas axon terminals and axons of L2 and L3 were imaged in the medulla, and dendrites of L2 and L3 and R1-6 terminals were imaged in the lamina.

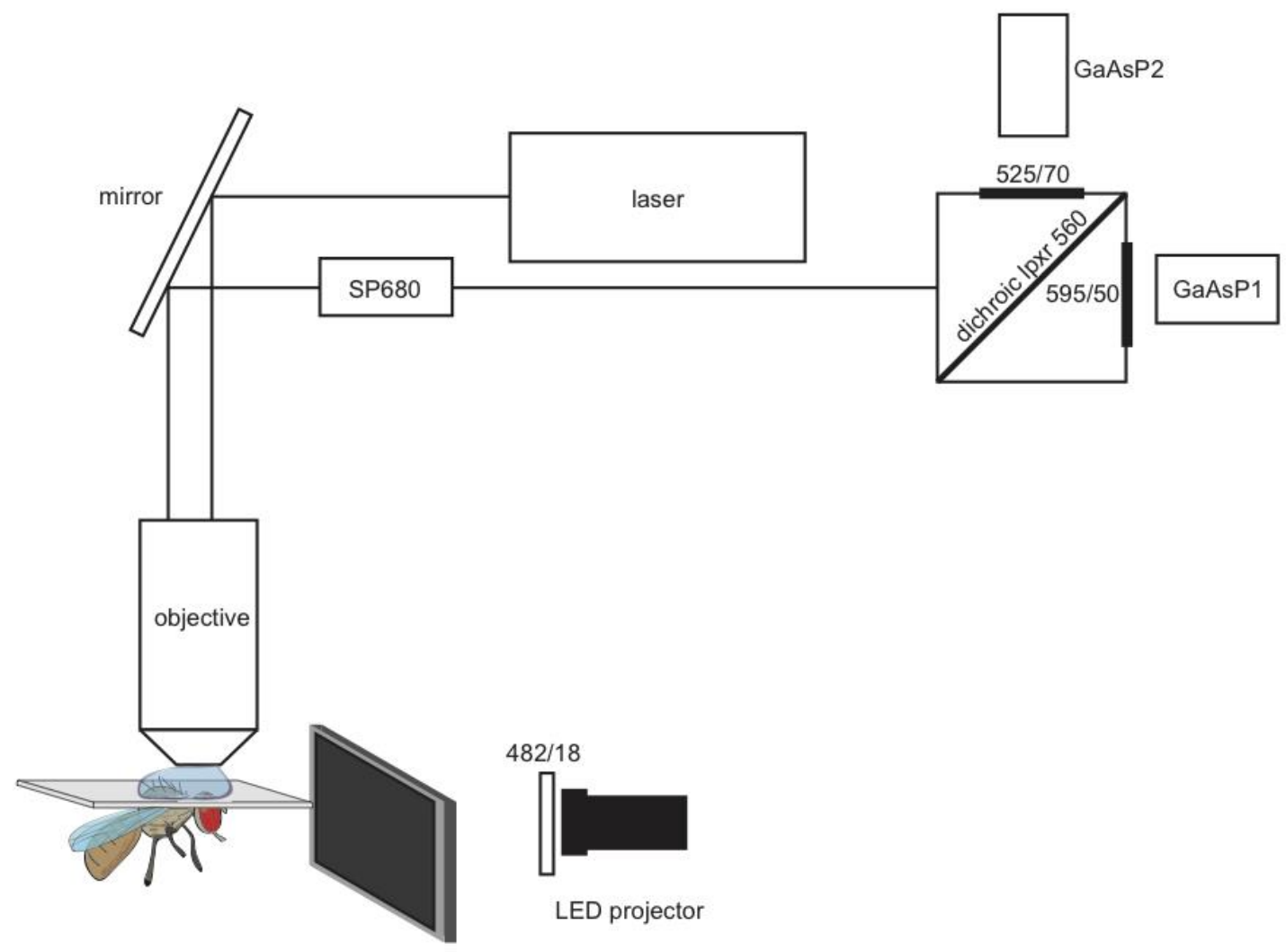


Figure 12. In vivo two-photon microscopy setup schematic

Schematic of the light path of the two-photon microscope and visual stimulation setup used in this study.

\subsubsection{Visual stimulation for calcium imaging}

Custom-written software using $\mathrm{C}++$ and OpenGL was used to generate visual stimuli. A LightCrafter (Texas Instruments, Dallas, TX, USA) running at a $100 \mathrm{~Hz}$ frame rate was used for the stimulus projection. Stimulus light travelled through a 482/18 band pass filter and a ND1.0 neutral density filter onto an $8 \mathrm{~cm} \times 8 \mathrm{~cm}$ rear projection screen. The projection screen was positioned in front of the animal and reached $60^{\circ}$ of the fly visual field in azimuth and $60^{\circ}$ in elevation (Figure 11). The visual stimuli used are described below. All contrast values were calculated using the definition of Weber contrast.

When testing the L2 and L3 contrast and luminance sensitivity across different adaptation states, we first empirically tested how bright the stimulus could be without getting any bleed through while imaging. This intensity was $15,75 \mathrm{~cd} / \mathrm{m}^{2}$ measured from the projection screen on the same side where flies look at the screen. In order to alter the overall mean intensity of the stimulus we used neutral density (ND, Thorlabs) filters ND 0.6 (25\% transmission) and ND 1.3 (5\% transmission). Therefore, intensity of the stimulus was $15,75 \mathrm{~cd} / \mathrm{m} 2$ without a filter, $3.84 \mathrm{~cd} / \mathrm{m}^{2}$ with the ND 0.6 and $0.91 \mathrm{~cd} / \mathrm{m}^{2}$ with the ND 1.3. The intensity of the stimulus used for all other experiments was 2.25 $\mathrm{cd} / \mathrm{m}^{2}$.

Full-field flashes: Periodic, alternating, 100\% contrast light and dark flashes covering the whole screen were presented to the flies. Flashes were each lasting $2 \mathrm{~s}, 5 \mathrm{~s}$ or $60 \mathrm{~s}$. Each $2 \mathrm{~s}$ and $5 \mathrm{~s}$ stimulus period was presented for $\sim 10$ trials, each $60 \mathrm{~s}$ period for $\sim 2$ trials.

Full-field flashes from an intermediate gray: Alternating light and dark flashes of fullcontrast and lasting for $2 \mathrm{~s}$ were interleaved by an intermediate gray epoch lasting for 4 $\mathrm{s}$ or light and dark flashes of $5 \mathrm{~s}$ were interleaved by an intermediate gray epoch lasting for $10 \mathrm{~s}$. Presentation of the ON or OFF flash from an intermediate gray was random. Each stimulus presentation period was presented for $\sim 5$ trials.

Different contrast steps from intermediate gray: The stimulus consisted of a $10 \mathrm{~s}$ gray period, followed by $5 \mathrm{~s}$ ON or OFF flashes of $20 \%, 40 \%, 60 \%, 80 \%$ or $100 \%$ contrast increments or decrements, relative to the intermediate gray background.

Flashes of different intensities: The stimulus consisted of $10 \mathrm{~s}$ full-field flashes of 5 different intensities (ranging from 0 to $100 \%$ of maximal intensity in $25 \%$ steps). The 
order between the periods was randomized, resulting in 20 different step combinations with varying Weber contrast.

Two OFF steps from adapted background stimulus: To distinguish contrast and luminance sensitivity, we designed a stimulus after (Oesch and Diamond, 2011). The stimulus consisted of a $30 \mathrm{~s}$ adapting period of $100 \%$ of max intensity, followed by two consecutive $3 \mathrm{~s}$ or $10 \mathrm{~s}$ OFF steps: the A and the B steps. The A step was one of 6 linear decreasing luminance values (Figure 13A), resulting in 6 different contrast steps (Figure 12B) relative to the adapting step. The luminance of the next OFF step, the B step, was one of 6 linear decreasing luminance values, depending on the previous A step, all of which resulted in 6 equally sized 25\% contrast steps (Weber contrast) (Figure 13B). The order of the different A steps and their associated B steps was randomized.

A

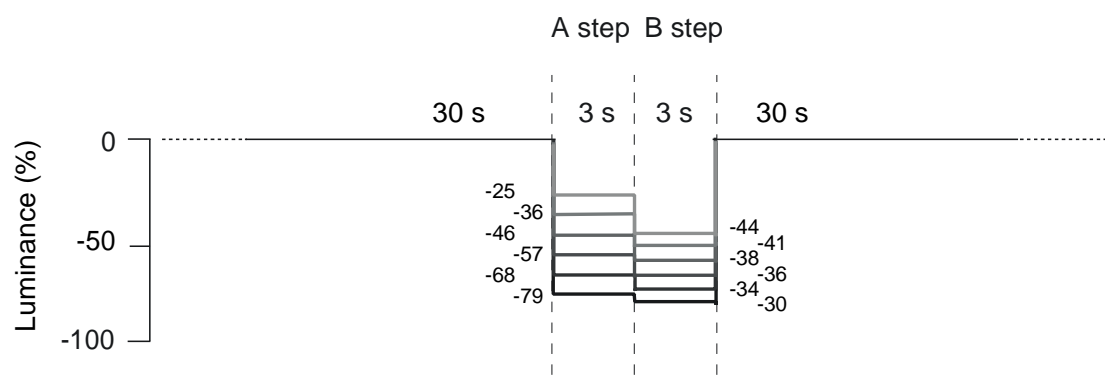

B

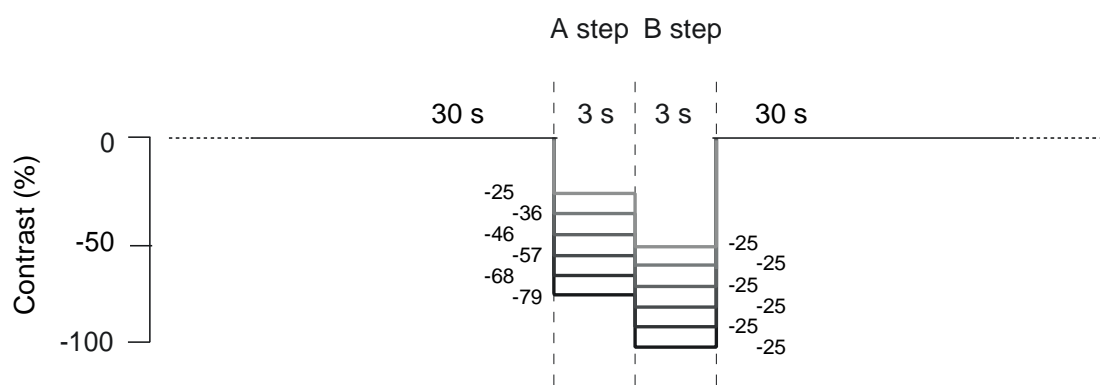

Figure 13. Schematic of the contrast steps from adapted background stimulus

(A-B) Schematic of the stimulus, consisting of an adapting bright period, followed by two sequential OFF steps is shown. (A) Luminance changes during the course of the stimulus are shown. (B) Contrast changes during the course of the stimulus are shown. Stimulus designed by Marvin Seifert.

Visual stimulation at different wavelengths: To test the spectral efficiency of L2 and L3, we used a multispectral light synthesiser based on LEDs and a diffraction grating (LED synthesizer (Belušič et al., 2016). The stimulus was passed through a $1 \mathrm{~mm}$ diameter 
light fiber and a collimating lens and was projected straight into the fly's compound eye. There, the stimulus subtended $\sim 25^{\circ}$. We presented the flies with light coming from a series of LEDs with peak wavelengths spanning from UV to red light. The peak wavelengths of LEDs used were $367,373,385,405,420,439,451,469,485,500,518$, $530,554,573,590,626,651,660,672$, and $682 \mathrm{~nm}$ (Figure 14A). The launching fiber has a limited aperture, thus the LED bandwidth was 10 nm. LED intensity was calibrated with a spectroradiometer (Flame, calibrated with a radiometric light source DH-3P-CAL; both Ocean Optics, USA). LED intensity was adjusted using 12-bit pulse width modulation at $1 \mathrm{kHz}$ to create an isoquantal spectral sequence (i.e. all LEDs had equal photon fluxes). Visual stimulation and PMT shutters were synchronized so that the PMT shutters were closed during the presentation of the light flash. We experimentally determined the shutters closing time to be $18 \mathrm{~ms}$ and shutters opening time to be $6 \mathrm{~ms}$, by measuring stimulus bleedthrough. Therefore, we programmed the imaging protocol using The Mark Points dialog in the Prairie View program. The imaging protocol was set up in in a way that the imaging computer sent a signal to close the shutters $18 \mathrm{~ms}$ before presentation of the flash and it sent the signal to open the shutters $6 \mathrm{~ms}$ before the stimulus end (Figure 14B). Doing so, the shutters were closed during the entire presentation of the stimulus flash and opened right after the end of the flash. For example, when we presented the flies with $2 \mathrm{~s}$ long flashes, we set up the duration of the shutters being closed to $1994 \mathrm{~ms} .1994 \mathrm{~ms}$ plus the opening time of the shutters resulted in exactly $2 \mathrm{~s}$ of shutters being closed. Thus, the full offset response was recorded and analyzed. Peak responses to each LED presentation were used to obtain spectral efficiency response curves. 
A

\begin{tabular}{|c|c|}
\hline LED & peak [nm] \\
\hline 1 & 367 \\
\hline 2 & 373 \\
\hline 3 & 385 \\
\hline 4 & 405 \\
\hline 5 & 420 \\
\hline 6 & 439 \\
\hline 7 & 451 \\
\hline 8 & 469 \\
\hline 9 & 485 \\
\hline 10 & 500 \\
\hline 11 & 518 \\
\hline 12 & 530 \\
\hline 13 & 554 \\
\hline 14 & 573 \\
\hline 15 & 590 \\
\hline 16 & 626 \\
\hline 17 & 651 \\
\hline 18 & 660 \\
\hline 19 & 672 \\
\hline 20 & 682 \\
\hline
\end{tabular}

B

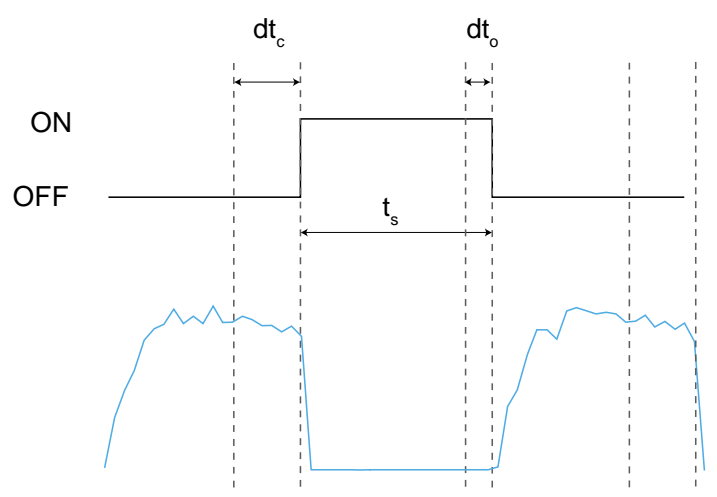

Figure 14. Stimulus setup of the LED synthesizer.

(A) List of LEDs with their peak wavelengths. (B) Schematic of the imaging protocol. Closing time of the shutters $\left(\mathrm{dt}_{\mathrm{c}}\right)$ is $18 \mathrm{~ms}$, whereas opening time of the shutters $\left(\mathrm{dt}_{\mathrm{o}}\right)$ is $6 \mathrm{~ms}$. In blue, example trace of one $\mathrm{L} 2$ neuron is shown. Note that the shutters are closed during the presentation of the flash and they open immediately after the end of the flash due to the fact that we took the shutter opening and closing times into an account.

\subsubsection{Two-photon calcium imaging analysis and statistical tests}

Matlab (Mathworks, Natick, MA) was used for data analysis and statistics. Crosscorrelation upon Fourier transformation was used to align the acquired images to a reference stack composed of a maximum intensity projection of the first 30 frames. Regions of interest (ROIs) were selected manually (Figure 15A,B) and an average intensity within individual ROIs was computed for each frame in order to obtain a time trace of the response of each ROI (Figure 15C). All responses and visual stimuli were interpolated to $10 \mathrm{~Hz}$, except $A S A P 2 \mathrm{f}$ voltage recordings, which were interpolated at 30 Hz. $d F / F\left(d F / F=\left(F-F_{0}\right) / F_{0}\right)$ was calculated (Figure 15D) and all responses were averaged across the repeating trials (Figure 15E). For all the stimuli, except for "visual 
stimulation at different wavelengths", single ROI responses from Tm9, L2 and L3 neurons were correlated with the stimulus and sorted by a correlation coefficient (Q). Therefore, if the calcium signal of one ROI increased to the light offset, this neuron was negatively correlated with the stimulus. Since Tm9, L2 as well as L3 increase in calcium signal to the darkness, only responses that were correlated with the stimulus with a negative sign (correlation coefficient $\mathrm{Q}<0$ ) were used for the analysis (Fisher et al., 2015). In contrast, for the analysis of photoreceptor calcium responses and ASAP $2 f$ responses, only positively correlated ROI responses with the stimulus were included. Mean responses were first measured for all ROIs within a fly, and then between flies (Figure 15F). All statistical analysis was performed between means per fly.

When analyzing responses obtained with "visual stimulation at different wavelengths", we manually selected flies. Only responding flies were included in the consequent analysis. Using the Mark Points function in the PrairieView software, the shutters are closed when the light is on. During these times, the fluorescence is zero. Manually setting a threshold, we extracted the epochs. This allowed us to calculate the duration of the stimulus presentation. Since there might be some fluctuations in the calcium response crossing the threshold value, we kept only the epochs, which were longer than 1 s. Since some of the recordings lasted for few seconds longer than the LEDs presentation (and thus shutters stayed open), we cut the calcium trace at the point where the duration of the epoch was longer than $4 \mathrm{~s}$. Calcium responses to the same LED presentation were then averaged. 
A

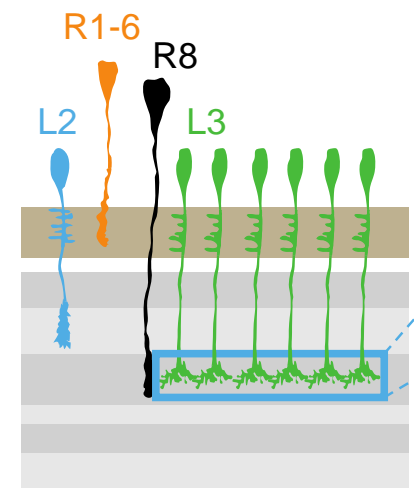

C

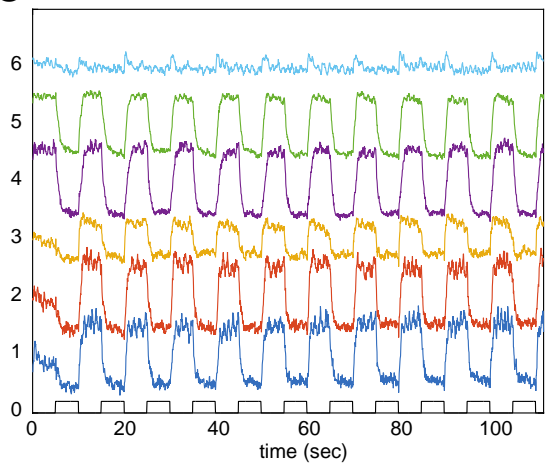

B

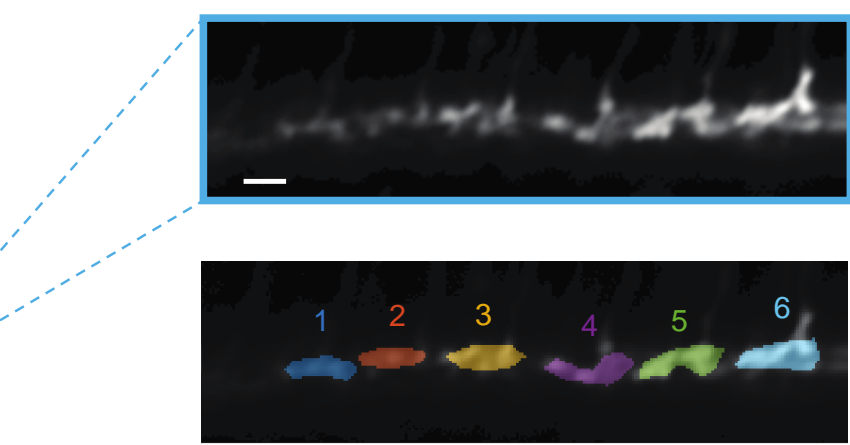

E
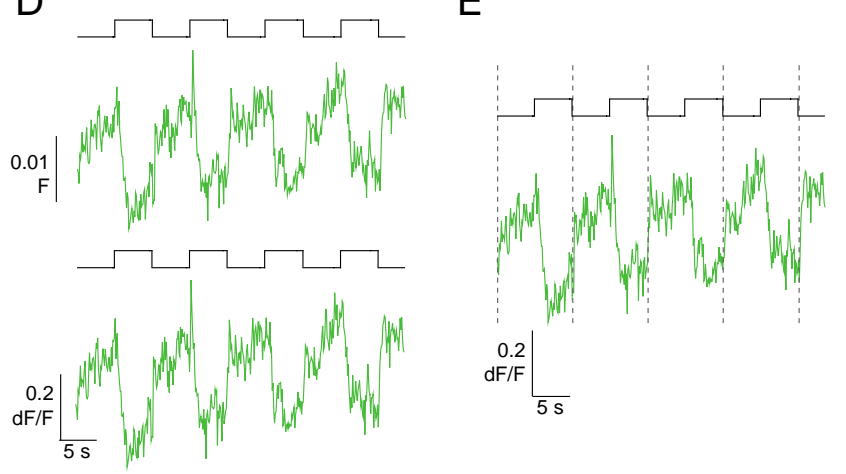

$\mathrm{F}$

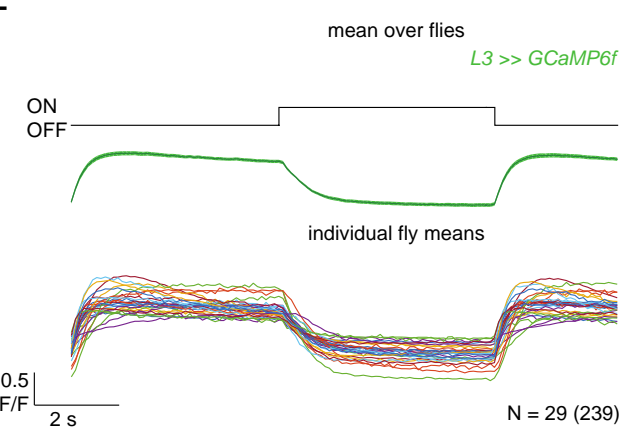

Figure 15. Step-by-step procedure of the calcium imaging analysis

(A) Schematic highlighting the two OFF pathway inputs L2 (blue) and L3 (green) and the photoreceptors R1-6 (orange) and R8 (black). Shaded L2 and L3 neurons illustrate the columnar organization of the visual system. The boxed area indicates a typical region for imaging. (B) The corresponding in vivo 2-photon images of rows of axon terminals are shown above. Bellow, regions of interest (ROIs) are selected manually and correspond to one axon terminal from one cell. Scale bar is $10 \mu \mathrm{m}$. (C) Time traces of the responses of each ROI are shown. (D) Above, calcium signal trace without calculating $\mathrm{dF} / \mathrm{F}$ is shown. Below, calcium signal trace after the $\mathrm{dF} / \mathrm{F}$ calculation is shown. (E) The data was segmented into epochs based on the repeating trials. Dashed line indicates the epochs. (F) Above, mean calcium responses were calculated over flies. Bellow, individual fly calcium signal means are shown.

Full field flashes: For calculating $\left.d F / F=\left(F-F_{0}\right) / F_{0}\right)$, the mean fluorescence intensity signal of the whole trace was used as $\mathrm{F}_{0}$. Peak calcium responses of $\mathrm{Tm} 9$ neurons were calculated as the maximal calcium response in the first $3 \mathrm{~s}$ after the OFF flash compared 
to the mean baseline response over the period of $2 \mathrm{~s}$ before the step. A two-tailed student $t$ test was used to test for statistical differences between peak amplitudes of $\mathrm{Tm} 9$ control and $\mathrm{Tm} 9$ neurons where lamina neurons were silenced.

Full-field flashes from an intermediate gray: For calculating $d F / F$, the mean fluorescence intensity signal of the trace to the gray period was used as the $F_{0}$. Peak OFF responses to the gray-to-OFF or ON-to-gray steps were calculated relative to the mean response over the $1 \mathrm{~s}$ before the step. A two-tailed student $t$ test was used to test for statistical differences between equivalent OFF steps.

Different contrast steps from intermediate gray: For calculating $d F / F$, the mean fluorescence intensity signal of the trace to the gray step was used as $F_{0}$. Peak OFF responses to the OFF- to-gray or ON-to-gray steps were calculated relative to the mean response over the $2.5 \mathrm{~s}$ before the step. A two-tailed student $t$ test was used to test for statistical differences between equivalent OFF steps.

Flashes of different intensities: For calculating $d F / F$, the mean fluorescence intensity signal of the trace to the $100 \% \mathrm{ON}$ step was used as the $F_{0}$. The plateau response was calculated as the maximal difference in the calcium signal at the last $2 \mathrm{~s}$ of the response compared to the mean baseline response during 2 seconds before the step. One-way analysis of variance (ANOVA) was used to test for differences between responses to different steps ending at the same intensity.

Contrast steps from the adapted background stimulus: To calculate $d F / F, F_{0}$ was calculated as the mean calcium response at the last $5 \mathrm{~s}$ of the $30 \mathrm{~s}$ adaptation period. Calcium responses were calculated either as the maximal calcium response in the first 3 $\mathrm{s}$ after the step (for both A and B step) compared to the mean baseline response over the period of $5 \mathrm{~s}$ before the step. When the A and B steps were $10 \mathrm{~s}$ long, calcium responses were calculated either as the maximal calcium response in the $10 \mathrm{~s}$ after the step (for both A and B step) or the mean calcium responses during the last $1 \mathrm{~s}$ of the A or B step compared to the mean baseline response over the period of $5 \mathrm{~s}$ before the step. Decay rates were calculated for the responses to the contrast steps from the adapted background stimulus. A linear regression model $(f(x)=a * x+b)$ was fit to the responses to the A step or to the A and B steps individually. One-way ANOVA was used 
to estimate whether responses to the B step were significantly different within the same genotype.

Visual stimulation at different wavelengths: To calculate $d F / F$, the mean fluorescence signal in response to $682 \mathrm{~nm}$ (red LED) was used as $F_{0}$. Maximum responses to individual LEDs were taken for the analysis. To test for statistical differences between L2 and L3 responses to different wavelengths, a two-tailed student $t$ test was used.

\subsection{PCR for verifying ort mutants}

To verify the existence of the $\operatorname{ort}[1]$ mutation, we performed a single fly PCR.

The primer pair combination was:

Ort1 seq forward: 5'- ACT TCC ATG TGA CGG TGA TG - 3'

Ort1 seq reverse: 5'- AAC ATG GTG GCT TGT TGC TT - 3'

\subsubsection{Single fly PCR protocol}

Sufficient amount of DNA can be obtained from one fly and the protocol is described below.

\subsubsection{Preparation}

- The squishing buffer (SB): $10 \mathrm{mM}$ TrisHCl with a pH 8.2, 1mM EDTA, 25mM $\mathrm{NaCl}, 200 \mu \mathrm{g} / \mathrm{ml}$ of Proteinase $\mathrm{K}$ with the enzyme diluted fresh from a 20 $\mathrm{mg} / \mathrm{ml} \mathrm{stock}$

- Primer concentration was adjusted to $2 \mu \mathrm{M}$

\subsubsection{Squishing Reaction Protocol}

- Each fly was placed in its own $0.5 \mathrm{ml}$ tube at $-20^{\circ} \mathrm{C}$ for about $15 \mathrm{~min}$

- Remove the fly from the freezer and mash the fly in the tube with a pipette containing $50 \mu \mathrm{l}$ of SB. When the fly is homogenized, expel the remaining SB

- Incubate for $30 \mathrm{~min}$ at $37^{\circ} \mathrm{C}$ and at $85^{\circ} \mathrm{C}$ for $2 \mathrm{~min}$

- $\quad 1-2 \mu \mathrm{l}$ will be used for PCR reaction, the rest can be stored at $4^{\circ} \mathrm{C}$

\subsubsection{PCR Master Mix}

Mix all the ingredients. The amount for one sample in $25 \mu \mathrm{l}$ of final volume is.

- $\quad 2 \mu \mathrm{l}$ of the squishing product

- $\quad 5 \mu \mathrm{l}$ Primer 1

- $\quad 5 \mu \mathrm{l}$ Primer 2

- $\quad 5 \mu \mathrm{l}$ Q5 buffer 
- $\quad 5 \mu \mathrm{l}$ Q5 enhancer

- $\quad 1 \mu \mathrm{l} 10 \mathrm{mM}$ dNTPs

- $\quad 1 \mu \mathrm{l} 25 \mathrm{mM} \mathrm{MgCl}_{2}$

- $\quad 0.25 \mu \mathrm{l}$ Q5 polimerase

- $\quad 0.75 \mu \mathrm{lddH} \mathrm{H}_{2} \mathrm{O}$

\subsubsection{PCR Protocol}

The samples were incubated in the thermocycler. The protocol used:

$98^{\circ} \mathrm{C}$ for $30 \mathrm{~s}$

30 cycles of:

$98^{\circ} \mathrm{C}$ for $10 \mathrm{~s}$

$60^{\circ} \mathrm{C}$ for $20 \mathrm{~s}$

$72^{\circ} \mathrm{C}$ for $50 \mathrm{~s}$

$72^{\circ} \mathrm{C}$ for $2 \mathrm{~min}$

$4^{\circ} \mathrm{C}$ for $\infty$

\subsubsection{PCR Agarose Gel}

To make $1 \%$ agarose gel.

- 1x TAE buffer

- $1 \mathrm{~g}$ of agarose

Mix agarose powder with $100 \mathrm{ml} 1 \mathrm{xTAE}$ and microwave until the agarose is completely dissolved.

- $\quad$ Add $3 \mu \mathrm{l}$ of ethidium bromide (EtBr)

Pour the gel into the holder and let it sit at room temperature until solid (20-30 min).

\subsubsection{Electrophoresis}

Add $5 \mu$ of loading buffer to each of the DNA samples. When the gel is solidified, place it into the electrophoresis unit. Load the $1 \mathrm{~kb}$ molecular ladder into the first well of the gel. Add the DNA samples into additional wells. Run the gel at $80 \mathrm{~V}$ and $400 \mathrm{~mA}$ until the dye is around $80 \%$ down the gel (approximately $40 \mathrm{~min}$ ). Remove the gel and using a UV light, visualize the DNA fragments. 


\section{RESULTS}

\subsection{Contrast and luminance sensitivity of L2 and L3}

In the visual system of a fly, distinct $\mathrm{ON}$ and OFF pathways separate downstream of photoreceptors. The two first order lamina interneurons L2 and L3 are the two main inputs to the OFF pathway. Although they are both downstream of the photoreceptor cells, they show different physiological properties. While L2 responds transiently to a sustained visual input, L3 neurons show sustained responses (Clark et al., 2011; Silies et al., 2013). Furthermore, it was shown that they are both required for behavioral responses to moving dark edges (Clark et al., 2011; Silies et al., 2013). Thus, we aimed to understand why such fundamentally different parallel OFF pathway inputs are needed and what the mechanisms are that initially shape them.

\subsubsection{L2 and L3 neurons show different physiological properties}

Responses at the initial processing stages in the fly visual systems have been extensively characterized (see reviews Borst, 2009; Silies et al., 2014; Yang and Clandinin, 2018). Fly photoreceptor cells respond to prolonged illumination with an initial transient phase, lasting several milliseconds (Brown and Blinks, 1974; Laughlin and Hardie, 1978), continued by a plateau, which lasts for the stimulus length. The initial transient phase encodes contrast, whereas the sustained plateau phase is luminance-sensitive (Brown and Blinks, 1974; Laughlin and Hardie, 1978). Downstream of photoreceptors, lamina monopolar cells (LMC) were shown to possess transient responses to sustained input, discarding the luminance information and signal contrast (Laughlin, 1989). While most studies generalized this and did not distinguish between LMC cell types (Dubs et al., 1981; Guy and Srinivasan, 1988; Järvilehto and Zettler, 1971; Laughlin, 1975; Laughlin and Hardie, 1978; Laughlin et al., 1987), some distinct LMC properties have been reported as well: Intracellular voltage recording from blowfly LMCs have shown a different type of LMC, L3, of which a resting potential is more negative compared to L1 or L2, input impedance is higher and it shows a more sustained plateau response to a sustained light compared to L1/L2-type LMCs (Rusanen and Weckström, 2016; Uusitalo et al., 1995). These properties were in line with calcium recordings, which showed that L3 responses show sustained responses to flashes and display a monophasic linear temporal filter (Silies et al., 2013). Therefore, we wanted to understand these differences between the two OFF pathway inputs L2 and L3. When we recorded calcium signals from the L2 and L3 terminals, we could confirm that L3 calcium signals are more sustained than L2 (Fisher et al., 2015a; Silies et al., 2013). Thus, we aimed to further 
characterize L2 and L3 responses to visual stimuli and test whether these two inputs to the OFF pathway care about different features of the visual scene.

We specifically expressed GCaMP6f in L2 or L3 and recorded calcium signals in response to light flashes in the axon terminals of these neurons using in vivo 2-photon imaging (Figure 16A-C). Both L2 and L3 showed an increase in calcium signal to the light offset $(\mathrm{OFF})$ and with a decrease to the light onset (ON). L2 showed transient responses to light flashes, whereas L3 responses were sustained over the course of stimulus presentation (Fisher et al., 2015, Figure 16B,C), even when we presented light flashes that lasted tens of seconds (Figure 16D). This is in line with previously reported stating that L3 displays monophasic linear temporal filter (Fisher et al., 2015a; Silies et al., 2013), matching sustained responses to light flashes, and that L2 displays a biphasic linear temporal filter (Clark et al., 2011), explaining transient responses to light flashes. During the $2 \mathrm{~s}$ flashes, the calcium trace measured from an L2 axon terminal was still decreasing as a response to the OFF step when the ON step was already shown to the fly (Figure 16C). In contrast, $5 \mathrm{~s}$ long flashes were long enough for the L2 response to return back to baseline (Figure 16B). Therefore, from now on, only data obtained with 5 $\mathrm{s}$ version of the full-flashes stimuli will be presented. Taken together, L2 and L3 show distinct physiological properties to the same visual input. L2 neurons show transient responses, whereas L3 responses show sustained responses to sustained visual input. This is in line with previously measured L2 and L3 linear temporal filters (Clark et al., 2011; Silies et al., 2013). The important characteristic of a filter is that a filter allows to pass certain things and to reject others. A cell with a biphasic filter can capture acute transitions of a stimulus while discarding the constant value, whereas a cell with a monophasic filter would follow the stimulus course and would extract the stimulus' slow components (Suh and Baccus, 2014). Together, this argues that L2 should be sensitive to changes in the visual stimulus, and be sensitive to contrast, whereas L3 might be sensitive to the most fundamental visual feature, luminance.

\subsubsection{L2, but not L3, calcium signals are contrast-sensitive}

A lot of previous work had established that all "LMC responses are inverted, amplified and more transient versions of photoreceptor responses" (Laughlin, 1989). Since the above-described data argue against this being true for L3, we next used our cell type specific genetic access to carefully test whether both L2 and L3 are indeed sensitive to contrast. To initially test the contrast sensitivity of L2 and L3 neurons we presented a stimulus consisting of intermediate grey flash lasting for $10 \mathrm{~s}$ followed by $5 \mathrm{~s}$ long $\mathrm{ON}$ or OFF steps, both $100 \%$ Weber contrast (Figure 16E,F). This stimulus contained two equivalent OFF contrast steps, when changing from gray to OFF, and when changing 
from ON back to gray. Recordings from the axon terminals showed that both L2 and L3 neurons responded with a calcium signal increase to the OFF step coming from the intermediate grey. As expected for contrast-sensitive neurons, L2 responded with similar amplitude to both the OFF step coming from ON to grey, and to the OFF step from gray to OFF (Figure 16E,F). Interestingly, L3 hardly responded at all to the ON-togrey step, and these responses were smaller than the response to the OFF step of the same Weber contrast, but stepping from the gray (Figure 16E,F). This suggested that L2 but not L3 neurons are contrast-sensitive.

A

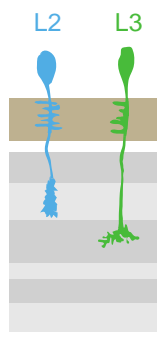

B
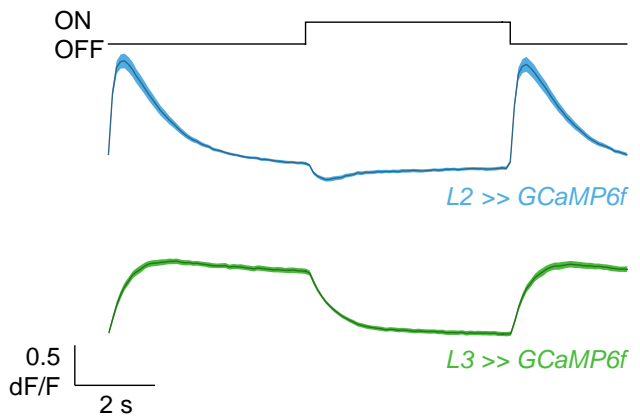

C
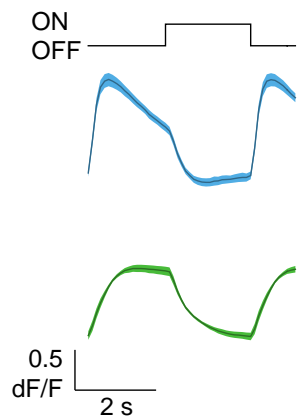

D

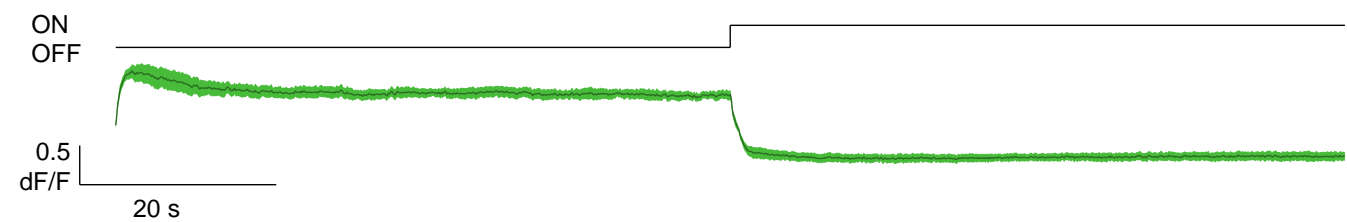

E
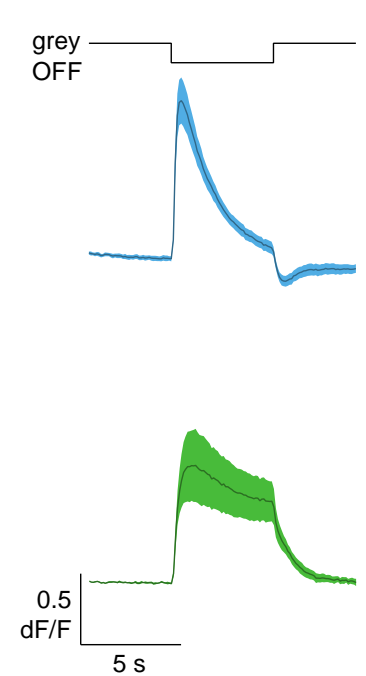
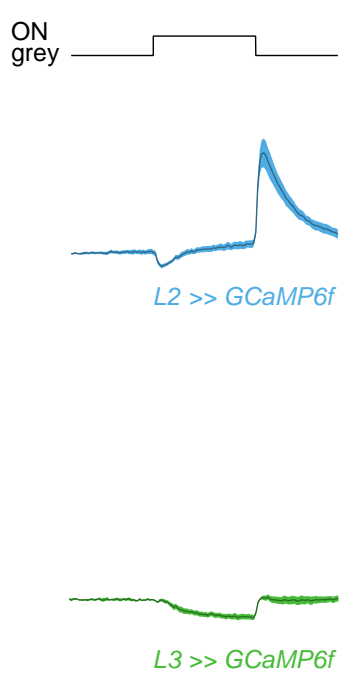

F
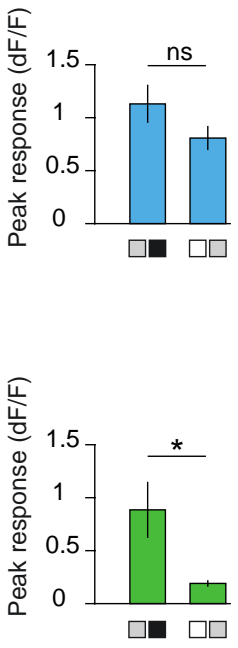

Figure 16. The two OFF pathway inputs L2 and L3 respond differently to visual inputs.

(A) Schematic showing the two OFF pathway inputs L2 (blue) and L3 (green) neurons. (B) GCaMP6f calcium signals from the axon terminals in response to $5 \mathrm{~s}$ full-field flashes of $100 \%$ contrast are shown in blue for L2 and green for L3. N = 23 flies (130 cells) for L2 and N = 20 flies (224 cells) for L3. (C) Calcium signals of 
L2 (blue) and L3 (green) axon terminals in response to $2 \mathrm{~s}$ full-field flashes of 100\%. N = 12 flies (213 cells) for $\mathrm{L} 2$ and $\mathrm{N}=7$ flies (120 cells) for L3. (D) Calcium signals of L3 axon terminals to $60 \mathrm{~s}$ full-field flashes. $\mathrm{N}=$ 5 (79) for L3. (E) Calcium signals measured from L2 (blue) and L3 (green) axon terminals in response to $5 \mathrm{~s}$ long ON or OFF flashes from a gray background. $\mathrm{N}=6$ flies (155 cells) for L2 and N = 5 flies (94 cells) for L3. (G) Bar plots showing the quantification of the peak responses of L2 (blue) and L3 (green) to the two OFF steps shown in (E): ON-to-gray and gray-to-OFF. ${ }^{*} \mathrm{p}<0.05$, tested with two-tailed Student $t$ tests. Squares at the bottom of $\mathrm{x}$-axis indicate the contrast step. All sample sizes are given as $\mathrm{N}=$ \#flies (\#cells). All traces and bars show mean \pm SEM.

To test the contrast sensitivity of L2 and L3 more thoroughly, we showed different ON or OFF steps, ranging from $20 \%$ to $100 \%$ Weber contrast, all relative to an intermediate gray background (Figure 17A,B). As described before, the stimulus contained two equivalent contrast steps, either changing from gray to OFF or changing from $\mathrm{ON}$ back to gray. Consistent with the results shown above, recordings from the axon terminals showed that L2 neurons responded to all contrast steps with a transient calcium signal. The response amplitude was greater for larger contrasts and decreased for smaller contrasts. L2 responded similarly to the OFF steps of the same contrast (Figure 17A,C). L3 responses also increased with increasing contrast to the gray-to-OFF step, but showed almost no response to the OFF step when returning to gray background after an ON step (Figure 17B,D). It can be noted though that responses of L2 neurons to ON to gray steps are always leaning towards having smaller amplitudes than L2 responses to gray to OFF steps. The ON step lasted for 5 seconds, which could be long enough for the adaptation process of the visual system to start. If this was the case, the flies would not be adapted to the stimulus' mean luminance anymore and therefore, the Weber contrast between gray-to-OFF and ON-to-gray would not be equivalent. However, L2 responses to the two OFF steps were not statistically different for any of the different contrasts tested. Together, our data show that L2 and L3 inputs respond very differently to simple visual inputs and suggest that calcium signals in L2 but not L3 neurons signal contrast changes. 
A
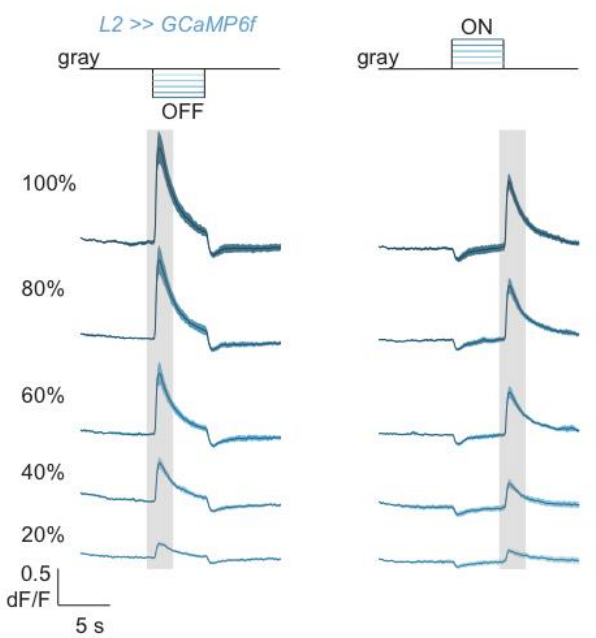

C

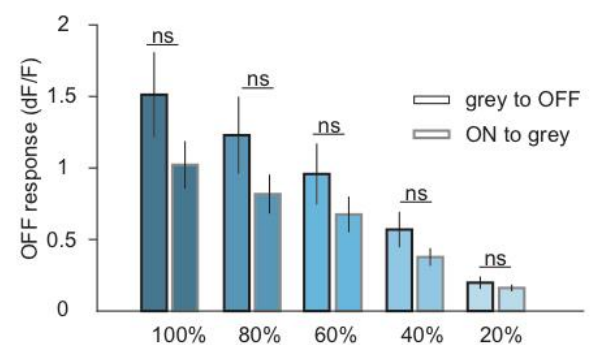

B

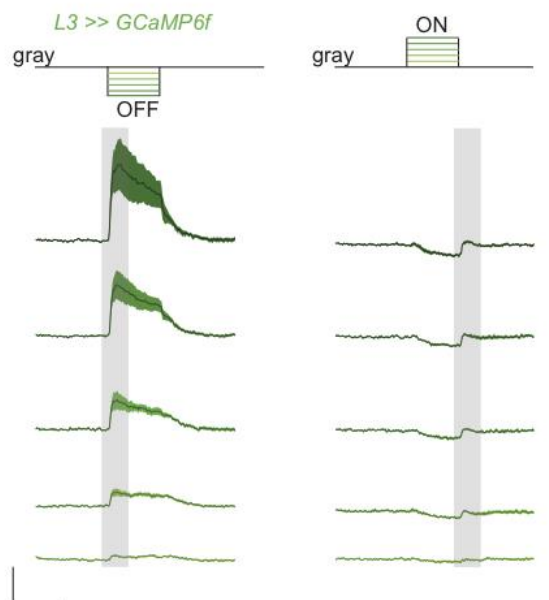

D

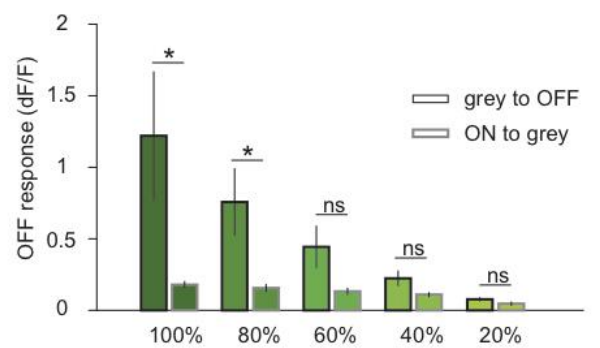

Figure 17. L2 neurons, but not L3 neurons are contrast-sensitive.

(A,B) Calcium signals in L2 (A) and L3 (B) axon terminals in response to full-field flashes of different Weber contrast, ranging from $20 \%$ to $100 \%$ relative to an intermediate gray background. Gray boxes mark the OFF responses. (C,D) Bar plots showing the quantification of the peak responses of the two OFF steps shown in $(A, B)$ : Bars with black outlines show responses to the gray-to-OFF steps, bars with gray outlines show responses to the $\mathrm{ON}$-to-gray steps. ${ }^{*} \mathrm{p}<0.05$, tested with two-tailed Student $t$ tests. $\mathrm{N}=6$ flies (158 cells) for L2 and $\mathrm{N}=5$ (93) for L3.

\subsubsection{L3 is luminance sensitive and particularly active in dim light}

L3 only responded to contrasts presented at dim background, but these stimuli also all differed in luminance. To explicitly test contrast versus luminance sensitivity in L2 and L3, we developed a stimulus based on a previous study describing luminance and contrast coding at the synapse between the bipolar and an amacrine cell in the vertebrate retina (Oesch and Diamond, 2011). Flies were first adapted to a $30 \mathrm{~s}$ long bright background and were then provided with two sequential OFF steps, A and B, in which the first OFF step A varied in magnitude with respect to both contrast and luminance and the second step B varied in luminance but Weber contrast was always 25\% (Figure 18A). Thus, differences in neuronal response to the first OFF step can be a result of changing either stimulus luminance or contrast. Since the second step is always 25\% Weber contrast, only contrast-sensitive cells will respond with the same magnitude. To allow the L2 calcium signal to return back to baseline, we used a stimulus 
in which the two OFF steps lasted for $10 \mathrm{~s}$. When plotting peak L2 responses as a function of a contrast, L2 scaled linearly with contrast to the first A step. This is not so informative as this contrast step varies in both contrast and luminance. Importantly, responses to the B step, which was always $25 \%$ Weber contrast, were indistinguishable from one another (Figure 18B). When plotting the same data as a function of luminance, we observed that responses to the B step, which was always 25\% Weber contrast, have the same amplitude, regardless of the luminance (Figure 18C). When we analyzed the mean response of the L2 neurons during the last second of the response to the A step, we observed that L2 always returned back to the same baseline, regardless of contrast or luminance (Figure 18D-F).

A

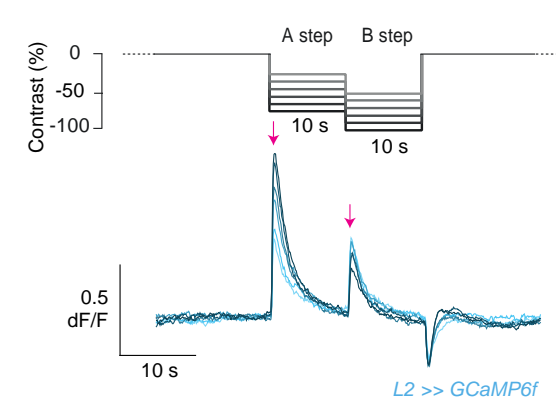

B

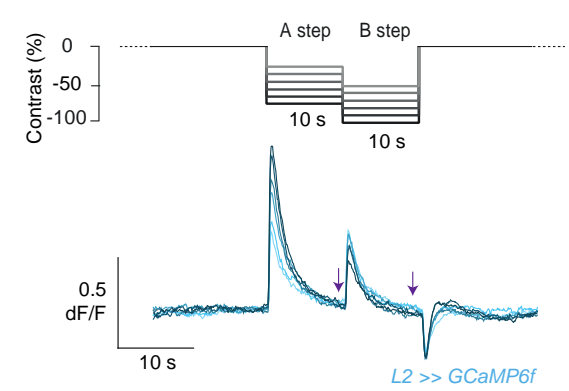

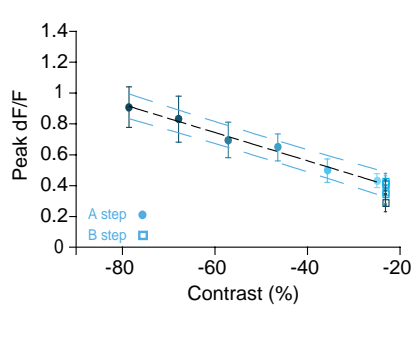

$\mathrm{E}$

E

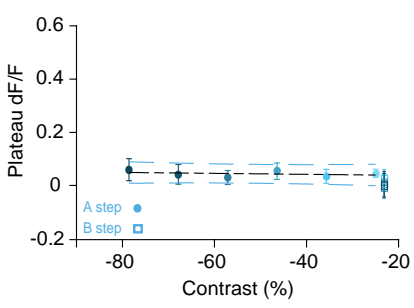

C

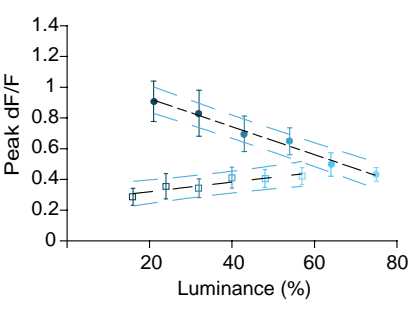

$\mathrm{F}$

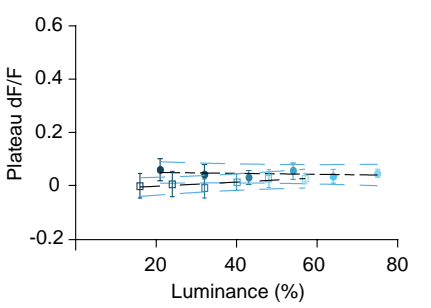

Figure 18. L2 neurons are contrast-sensitive.

(A) Schematic of the stimulus, consisting of a $30 \mathrm{~s}$ adapting bright period, followed by two sequential OFF steps, each $10 \mathrm{~s}$ long. The size of the step is illustrated by the gray-scale of the trace. Average calcium signals of L2 in response to the stimulus shown above. Darker traces correspond to OFF steps of larger amplitude. Arrows indicate the peak L2 responses, which were taken for the consequent analysis. (B,C) Peak calcium responses of $\mathrm{L} 2$ neurons plotted as a function of contrast (B) or luminance (C). A linear regression model (black dashed line) was fit to the responses to the A step (B) or to the A and B steps individually (C). The dashed lines indicate the $95 \%$ confidence interval. Responses to the A step are illustrated by circles, responses to the B step by squares. (D) Schematic of the stimulus, as in (A). Average calcium signals of L2. Arrows indicate the plateau L2 responses, which were taken for the consequent analysis. (E,F) Plateau calcium responses of L2 neurons plotted as a function of contrast (E) or luminance (F). A linear regression model (black dashed line) was fit to the responses to the A step (E) or to the A and B steps individually (F). The dashed lines indicate the $95 \%$ confidence interval. Responses to the A step are illustrated by circles, 
responses to the B step by squares. Significance was tested with one-way ANOVA $(p<0.05) . N=7(78)$ in $(A-$ F).

We then tested L3 neuron's responses to this stimulus. Calcium traces of L3 neurons to the $10 \mathrm{~s}$ long A and B flashes showed an initial increase in calcium signal, which then decayed to a certain plateau level (Figure 19A). When analyzing this initial peak response to the A step, calcium responses of L3 neurons to the A step scaled linearly with contrast and peak responses of L3 neurons to the same six $25 \%$ contrast changes trended to go down with increasing luminance, but were not statistically different from each other (Figure 19B-C). When plotting calcium signals as a function of luminance, L3 peak responses to the A step were not the same as the responses to the B step at a similar luminance (Figure 19C). This suggests that L3 peak responses are not luminance sensitive, but carry at least some information about contrast.

However, when analyzing plateau response of L3 neurons, L3 plateau responses to the same six 25\% contrast changes were no longer the same (Figure 19D,E). Plotting calcium signals as a function of luminance revealed that L3 plateau responses to the $\mathrm{A}$ step were similar as the responses to the B step at a similar luminance. Thus, L3 plateau responses are luminance-sensitive. To sum, this indicates that L3 has a peak response, which carries some contrast information and this peak response is followed by a plateau response, which is sensitive to luminance. 
A

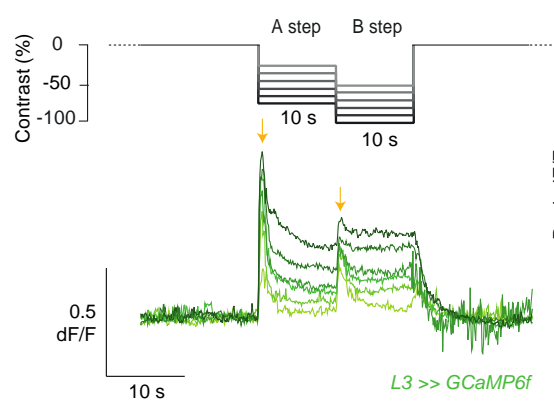

$\mathrm{D}$

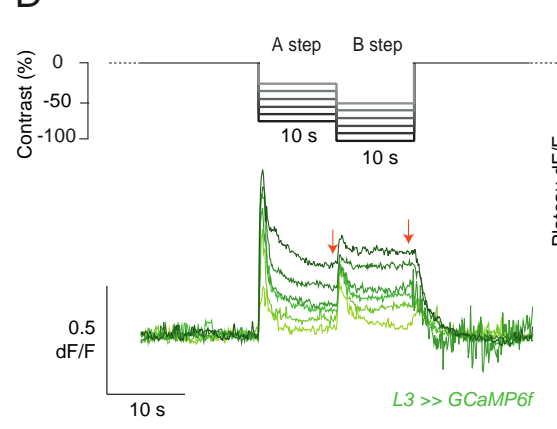

B

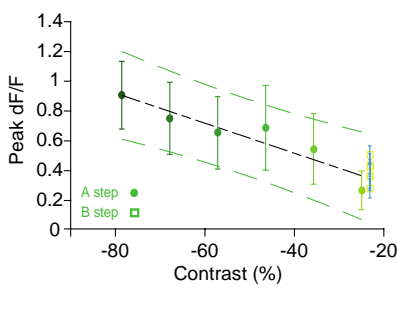

E

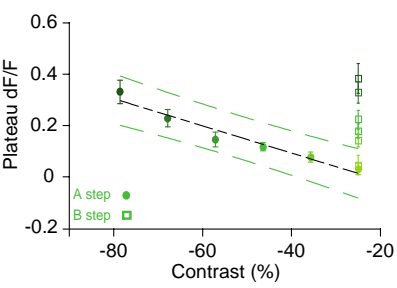

C

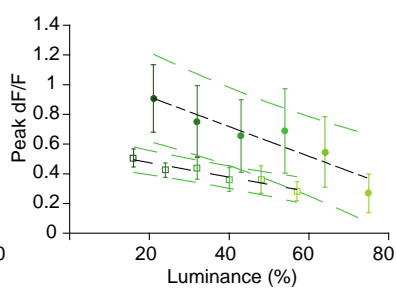

$\mathrm{F}$

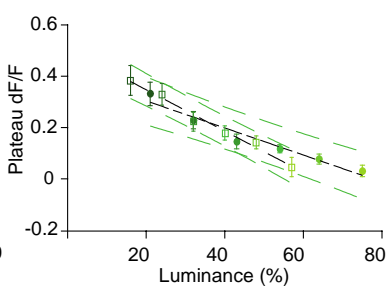

Figure 19. L3 is luminance-sensitive.

(A) Schematic of the stimulus, consisting of a $30 \mathrm{~s}$ adapting bright period, followed by two sequential OFF steps, each $10 \mathrm{~s}$ long. The step size is illustrated by the grayscale of the trace. Average calcium signals of L3 in response to the stimulus shown above. Darker traces correspond to OFF steps of larger amplitude. Arrows indicate the peak L3 responses, which were taken for the consequent analysis. (B,C) Peak calcium responses of $\mathrm{L} 3$ neurons plotted as a function of contrast (B) or luminance (C). A linear regression model (black dashed line) was fit to the responses to the A step (B) or to the A and B steps individually (C). The dashed lines indicate the $95 \%$ confidence interval. Responses to the A step are illustrated by circles, responses to the B step by squares. (D) Schematic of the stimulus, as in (A). Average calcium signals of L3 in response to the stimulus. Arrows indicate the plateau L3 responses, which were taken for the consequent analysis. (E,F) Plateau calcium responses of L3 neurons plotted as a function of contrast (E) or luminance (F). A linear regression model (black dashed line) was fit to the responses to the A step (E) or to the A and B steps individually (F). The dashed lines indicate the 95\% confidence interval. Responses to the A step are illustrated by circles, responses to the B step by squares. Significance was tested with one-way ANOVA $(\mathrm{p}<0.05) \mathrm{N}=10(93)$ in $(\mathrm{A}-\mathrm{F})$.

To minimize adaptation during the presentation of the A step, we also used a version of the same stimulus in which we only presented the two OFF steps for $3 \mathrm{~s}$. Data were recorded by Marvin Seifert, a MSc student in the lab. Calcium traces of L2 neurons to the 3 s long A and B flashes showed an initial transient increase in calcium signal, followed by decay in calcium signal, which is in line with the previous result (Figure 18A, 20A-B). Analysis of L2 peak responses to the B step showed contrast-sensitivity of L2 neurons (Figure 20A-E). Interestingly, in Marvin's hands, L3 neurons behaved slightly different 
and did not show a pronounced peak (Figure 20F). Analysis of these initial L3 responses showed that initial calcium responses in L3 are sensitive to luminance but not contrast (Figures 20G-I). This discrepancy between experiments is interesting because the genotype of the flies used and both experiments is the same. One reason for the discrepancy might be that the epoch duration of the A and B step influences the shape of peak responses of the L3 neurons. The second reason might be that the stimulus presentation history influences responses. Maybe Marvin showed several different stimuli to the flies before he presented them with this $3 \mathrm{~s}$ long version of the stimulus. The flies used in my experiment saw only this stimulus. However, analyzing plateau response revealed luminance-sensitivity of L3 in both experiments. This indicates that L3 does have a peak response in certain regimes and this peak response carries a contrast-sensitive component. However, the plateau response is sensitive to luminance but not contrast.

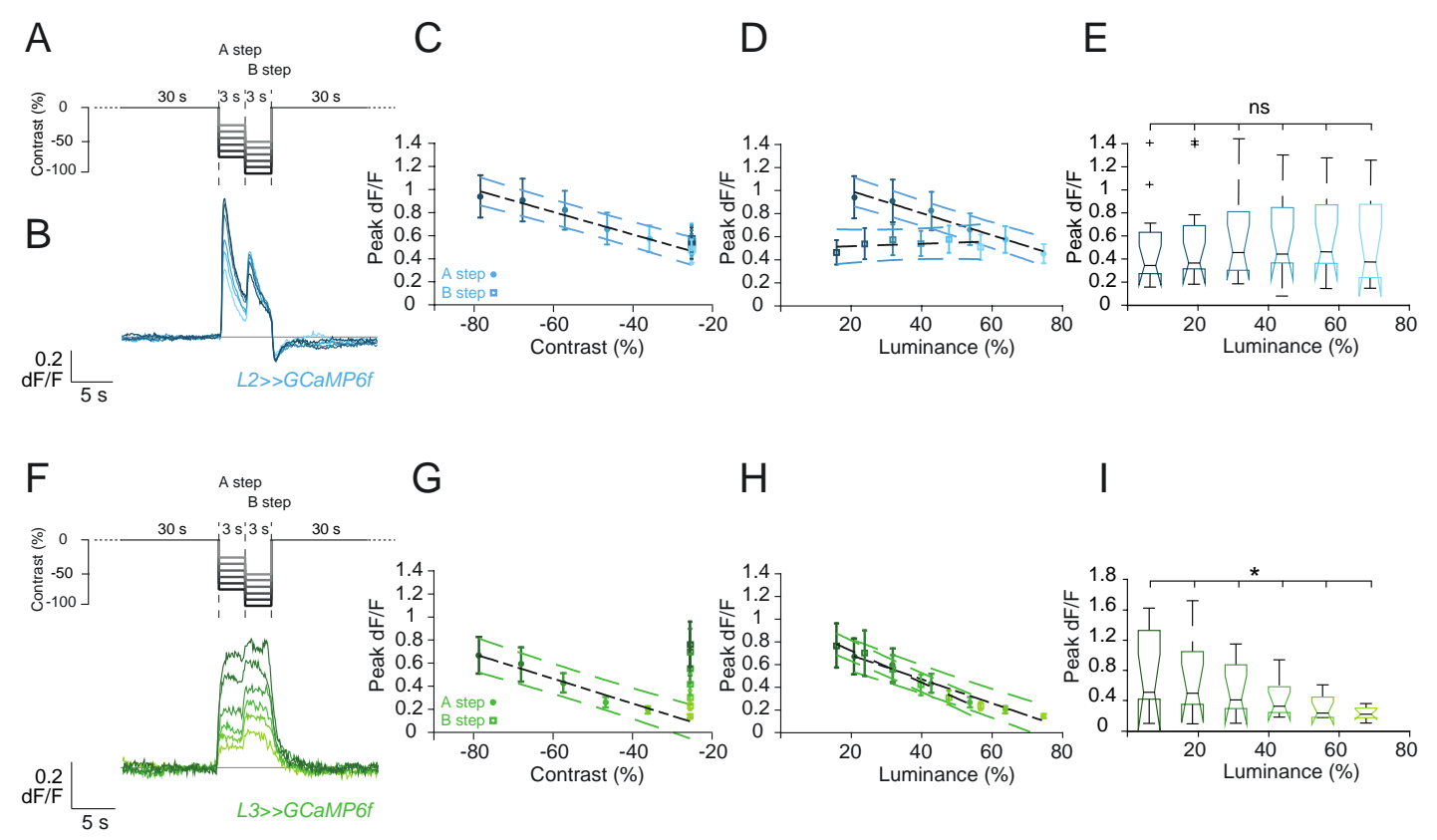

Figure 20. L2 peak responses are contrast-sensitive, whereas L3 peak responses are sensitive to luminance.

(A) Schematic of the stimulus, consisting of an adapting bright period, followed by two sequential OFF steps. The light intensity of the step is illustrated by the gray-scale of the trace. (B,F) Average calcium signals of L2 (B) and L3 (F) in response to the stimulus shown in (A). Darker traces correspond to OFF steps of larger amplitude. (C-D, G-H) Peak calcium responses of L2 (C-D) and L3 (G-H) neurons plotted as a function of contrast $(\mathrm{C}, \mathrm{G})$ or luminance $(\mathrm{D}, \mathrm{H})$. A linear regression model (black dashed line) was fit to the responses to the A step $(C, G)$ or to the A and B steps individually (D,H). The dashed lines indicate the $95 \%$ confidence interval. Responses to the A step are illustrated by circles, responses to the B step by squares. (E,I) Boxplot showing L2 (E) and L3 (I) peak calcium responses to the B steps. ${ }^{*} \mathrm{p}<0.05$, tested using ANOVA. N = 10 (132) for L2 and N = 10 (126) for L3 in (B-E). Data were acquired by Marvin Seifert. 
While the stimulus used above varies luminance while fixing contrast, we next tested the response of L2 and L3 (Figure 21A) to the same luminance values, but associated with different contrast changes. We presented the flies with a stimulus that varied randomly between five different light intensities, each lasting $10 \mathrm{~s}$ (Figure 21B). As seen in the responses of single axon terminals, calcium responses of L2 scaled with changes in intensity but returned to one fixed baseline after each contrast change (Figure 21B). In contrast, calcium signal recorded from L3 axon terminals were the same when the stimulus was reaching the same luminance, and did not depend on the contrast. Furthermore, L3 signals did not return to one fixed baseline (Figure 21B). Furthermore, L3 neurons were the most active at the lowest luminance (Figure 21B). To quantify this across many cells in many different flies, we first analyzed the peak responses of L2 and L3 neurons to the three steps with the same Weber contrast, but ending at different luminance: $100 \%$ ON to gray, $50 \%$ ON to $50 \%$ OFF and gray to $100 \%$ OFF (Figure 21C). Amplitudes of L2 calcium responses to these three OFF steps were not statistically significant (Figure 21D), confirming that L2 are contrast-sensitive irrespective of the luminance. In contrast to L2, L3 did not respond equally to these steps, but showed the strongest calcium signal to the darkest OFF contrast step (gray to $100 \%$ OFF), confirming that L3 neurons are most active in the lowest luminance.

For the analysis of the plateau component of the response we quantified responses during the last two seconds of the epoch. Analysis of the plateau response of L2 during this time window confirmed that the L2 baseline returned to zero for all epochs, regardless of the preceding step (Figure 21E-F). In contrast, plateau responses of L3 were indistinguishable from each other whenever the stimulus ended on a given luminance, and regardless of the step preceding it. Thus, L3 responses were luminance dependent and independent of the contrast step (Figure 21G). Therefore, responses to the steps ending at the same luminance were pooled together. This analysis confirmed that L3 was most active in dim light and showed a non-linear decline in signal amplitude with increasing amplitude (Figure 21H). 
A

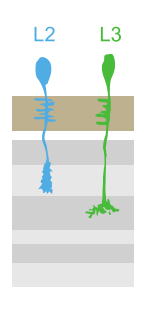

C

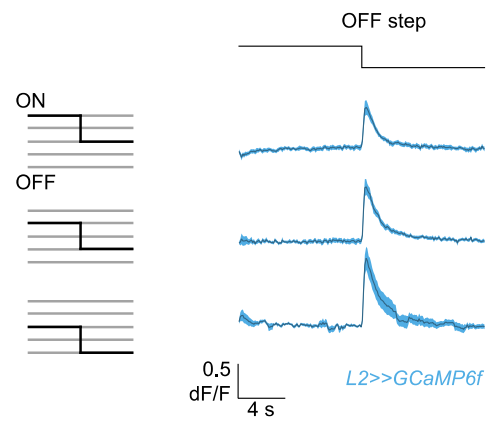

E

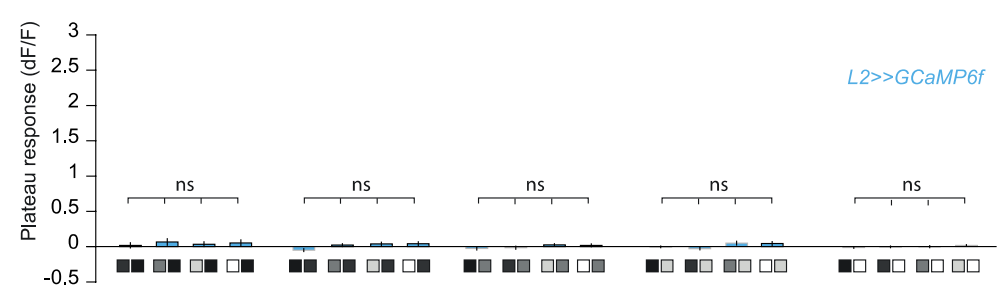

G

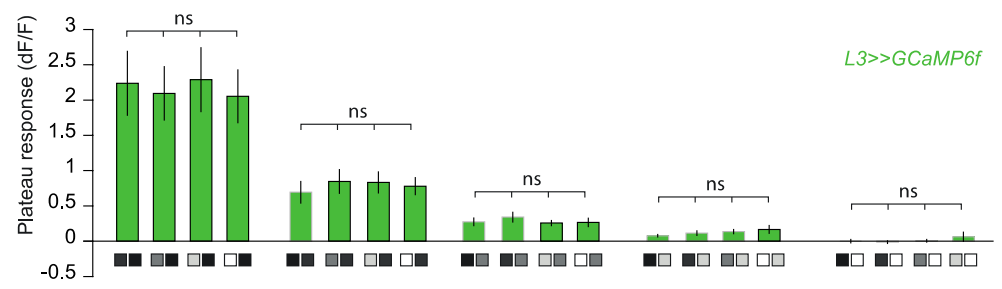

B

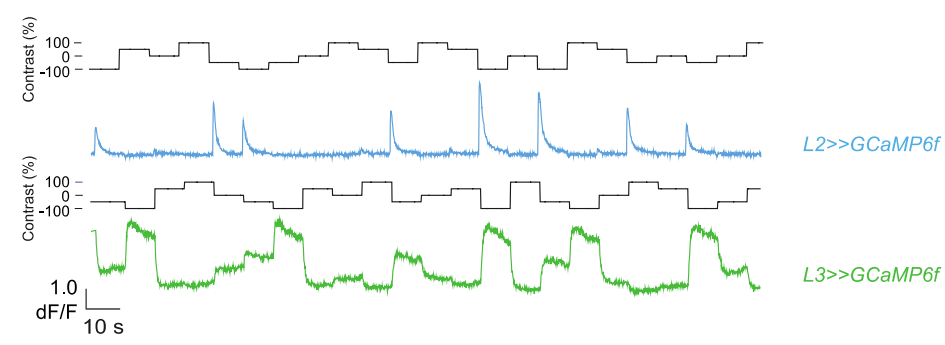

D

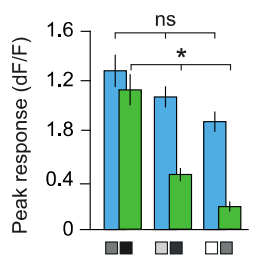

F

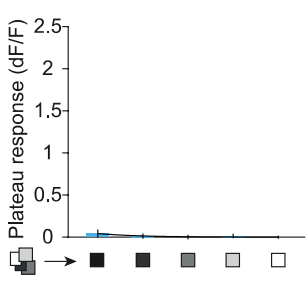

$\mathrm{H}$

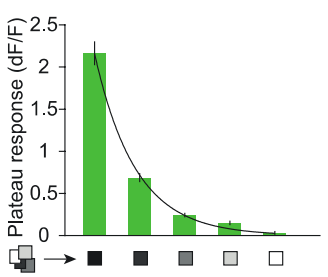

Figure 21. $\mathrm{L3}$ is luminance-sensitive.

(A) Schematic showing lamina neurons L2 (blue) and L3 (green). (B) Example calcium trace of single L2 (blue) or L3 (green) axon terminals to a stimulus comprising $10 \mathrm{~s}$ full-field flashes varying randomly between five different intensities. (C) Calcium signals of L2 (blue) and L3 (green) axon terminals in response to the three different steps varying in luminance, but of the same contrast: bright (top), middle luminance regime (middle) and dark luminance regime (bottom). $\mathrm{N}=10(226)$ for $\mathrm{L} 2$ and $\mathrm{N}=10(245)$ for L3. (D) Bar plots showing the L2 (blue) or L3 (green) peak responses to the OFF steps shown in (C). ${ }^{*} \mathrm{p}<0.05$, tested with one-way analysis of variance (ANOVA). $\mathrm{p}=0.070$ for L2 and $\mathrm{p}=0.028$ for L3. (E,G) Bar plot showing the L2 (E) and L3 (G) plateau responses measured for all steps ending at $-100 \%$ OFF, $-50 \%$ OFF, gray, $50 \% \mathrm{ON}$ and $100 \% \mathrm{ON}$, calculated as the average response across the last $2 \mathrm{~s}$ of stimulus presentation. Gray lines surrounding the bars represent the ON steps and black lines represent the OFF steps. ANOVA was used to test for differences between responses to different steps ending at the same intensity $(\mathrm{p}<0.05)$. Squares at the bottom of $\mathrm{x}$-axis indicate the step size. $\mathrm{N}=26(436)$ for $\mathrm{L} 2$ and $\mathrm{N}=$ 
31(512) for L3. (F,H) Bar plots showing the L2 (F) and L3 (H) plateau responses pooled for all steps ending at a given luminance. $\mathrm{N}=26$ (436) for $\mathrm{L} 2$ and $\mathrm{N}=31$ (512) for $\mathrm{L} 3$.

It should be noted that, when calculating Weber contrast, one needs to take into account the adaptation state of the animal. Our above calculation assumes that the flies are adapting to the mean luminance of the stimulus presentation:

$$
c=\left(\frac{I_{\text {after step }}-I_{\text {before step }}}{I_{\text {mean }}}\right) \text {. }
$$

However, we know that luminance adaptation is present in photoreceptors and can be fast, occurring within tens of milliseconds, and slow, taking tens of seconds (Adelson, 1982; Calvert et al., 2002; Dowling and Ripps, 1972; Fain, 1976; Laughlin, 1989; Laughlin and Hardie, 1978). During the $10 \mathrm{~s}$ lasting epochs we might be giving the flies a chance to already adapt to the new luminance. If this was the case, then the Weber contrast between the individual steps were:

$$
c=\left(\frac{I_{\text {after step }}-I_{\text {before } \text { 回ep }}}{I_{\text {before step }}}\right) .
$$

Given such adaptational contraints, it is even more striking that L2 responses to the same steps sizes are identical, and that L3 responses to the same luminance are indistinguishable from each other.

Taken together, our data show that two major inputs to the OFF pathway care about different features of the visual scene: calcium signals in L2 carry information about contrast and L3 neurons are luminance-sensitive.

\subsubsection{L3 neurons carry contrast information in their fast voltage response}

L3 responses seem to carry both contrast and luminance information in their peak and plateau response component, respectively (Figure 22A-B). However, calcium indicators might not be fast enough to accurately follow the fast calcium changes. Furthermore, intracellular recordings from L3 neurons have shown that L3 responses contain a fast contrast-sensitive response, which depends on the stimulus brightness (Uusitalo et al., 1995). Thus, we wanted to test the voltage signals of L2 and L3 neurons. To probe for this fast response under our stimulus conditions, we optically recorded voltage signals using the sensor ASAP2f (Figure 22C) (Yang et al., 2016). Unlike calcium indicators, many of the genetically encoded voltage indicators become dimmer upon depolarization (Figure 22C) (Yang and St-Pierre, 2016). Using cell-type-specific driver lines, we expressed ASAP2f specifically in L2 or L3. We then recorded voltage signals in response to light flashes in the axon terminals of these neurons. Both L2 and L3 transiently depolarized transiently to the OFF and hyperpolarized in a transient manner to the $\mathrm{ON}$ 
flash, consistent with previous LMC voltage recordings that were assigned to these cell types (Rusanen and Weckström, 2016; Uusitalo et al., 1995). This argues that even for stimulus conditions in which we did not detect a fast calcium transient, L3 shows a fast voltage response, consistent with previous electrophysiological recordings. Thus, L3 neurons do carry a fast contrast information in their initial response.

A

CALCIUM

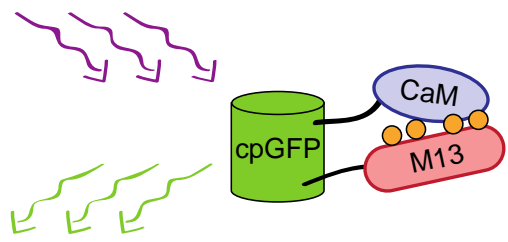

C

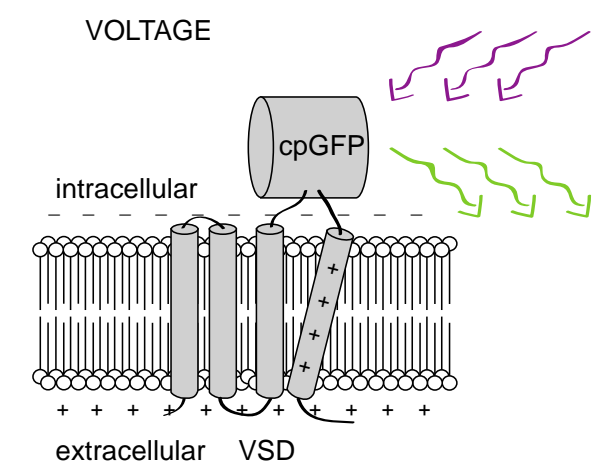

B

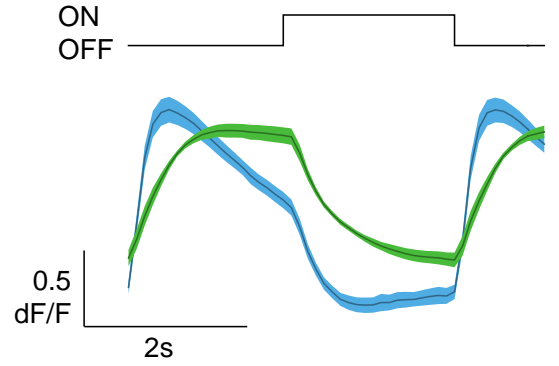

$\mathrm{D}$

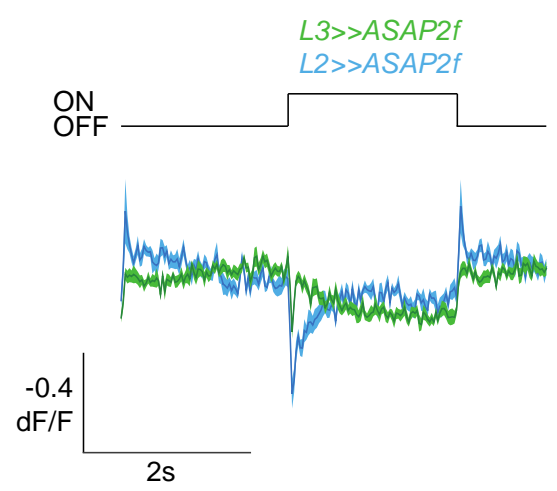

Figure 22. L3 voltage signals reveal fast contrast-sensitive responses

(A) Schematic of the GCaMP6 calcium indicator. GCaMP6 is composed of a cpGFP, which is positioned between CaM and an M13. Calcium binding causes CaM-M13 complex conformational changes, which induces cp-GFP fluorescence. GCaMP6 schematic modified after (Broussard et al., 2014). (B) GCaMP6f calcium signals from the axon terminals in response to $2 \mathrm{~s}$ full-field flashes of $100 \%$ contrast are shown in blue for L2 and green for L3. N = 12 flies (213 cells) for L2 and N = 7 flies (120 cells) for L3. (C) Schematic of an ASAP voltage sensor. Membrane potential changes cause a VSD transmembrane helix movement. This causes a change in the cp-GFP fluorescence. ASAP schematic modified after (Yang et al., 2016) (D) Average voltage signals (dF/F) recorded from L2 (blue) and L3 (green) axon terminals. Sample sizes are N = 11(72) for L2 and $\mathrm{N}=14(74)$ for L3.

\subsubsection{L2 neurons are contrast-sensitive in a wide dynamic range of overall luminance}

Calcium recordings showed that L2 neurons are contrast-sensitive, whereas L3 neurons are luminance-sensitive and mainly active in dim light. So far, we tested this for one 
adaptation state. We wanted to know whether L2 and L3 accurately report contrast or luminance at different adaptation states, represented by different mean light intensities. To test this, we presented the flies with stimuli while altering the overall mean intensity of the stimulus using neutral density (ND) filters (Figure 23A-C). An ND1 filter for example transmits $10 \%$ of photons, and thus dims the mean intensity by 10 -fold. We first set the stimulus to brighter intensity, resulting in a 7-fold intensity increase (see Material and Methods). After presentation of a set of stimuli we then positioned either ND 0.6 or ND 1.3 in front of the stimulus projector. ND 0.6 had 25\% transmittance, whereas ND 1.3 had 5\% transmittance (see Material and Methods) Using ND filters with higher strength than 1.3 resulted in very small L2 and L3 responses. Therefore, we did not use ND filters stronger than 1.3.

We then presented the flies with the stimulus that varied randomly between five different light intensities, each lasting $10 \mathrm{~s}$ (as in Figure 21B). L2 calcium responses to the OFF steps in all three light regimes showed a transient peak response, which returned to the same baseline. No changes in response kinetics were observed (Figure 23D,F,H) when the mean luminance was reduced using ND filters. When plotting peak L2 responses to the $100 \%$ ON to gray, L2 neurons responded with the same amplitude in all three luminance regimes (Figure 23D,E). The same was true for the other two contrast steps of same magnitude (50\% ON to $50 \%$ OFF and grey to $100 \%$ OFF). L2 responses were not significantly different across the three luminance regimes (Figure 23F-I). This suggests that the flies are adapted to the mean luminance and L2 neurons responded the same to the same contrast, regardless of the mean luminance.

To further test this, we presented the flies with an adapting period, followed by two sequential OFF steps as described above (Figure 20A) for all three light regimes. Interestingly, L2 calcium responses had the smallest amplitude for all the steps in the brightest regime (Figure 23J) and the biggest amplitude in the darkest luminance regime (Figure 23L). The reason might be that the stimulus in the brightest regime (no ND) is too bright and the fly's visual system reached saturation. However, despite smaller amplitude of the responses we could still observe that calcium responses of L2 neurons scaled linearly with contrast for all light regimes tested. Furthermore, for all three light regimes, all six responses to $25 \%$ contrast OFF steps were virtually indistinguishable from one another (Figure 22M-S). When we instead plotted responses as a function of luminance, L2 responses to close-by luminances differed from each other. Together, this confirms that L2 neurons are contrast-sensitive across different adaptation states. 
A

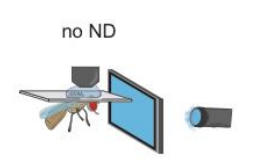

B

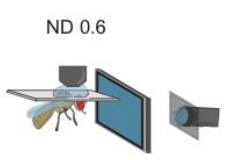

D
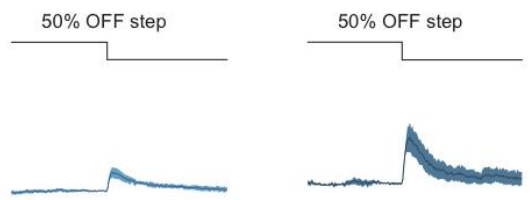

$\mathrm{F}$
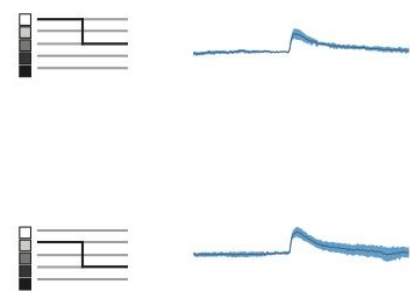

$\mathrm{H}$
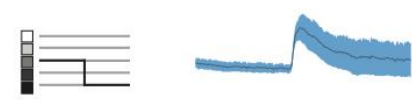

$\underset{\mathrm{dF} F \mathrm{~F}}{0.5} \frac{\mathrm{L}}{2 \mathrm{~s}}$

L2 >> GCaMP $f f$

$J$
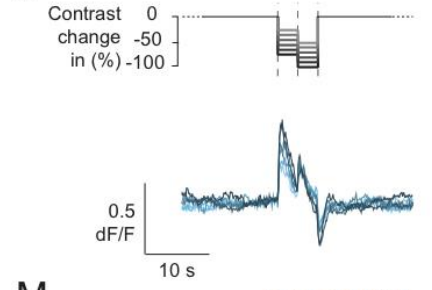

M
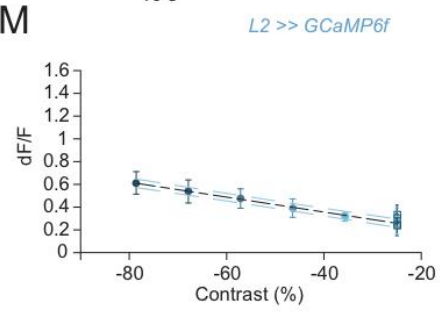

$\mathrm{P}$

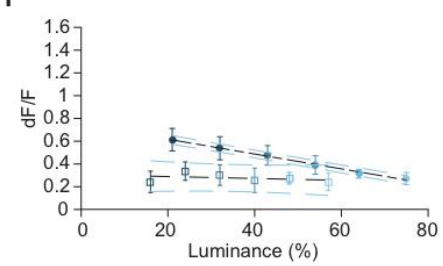

$\mathrm{K}$

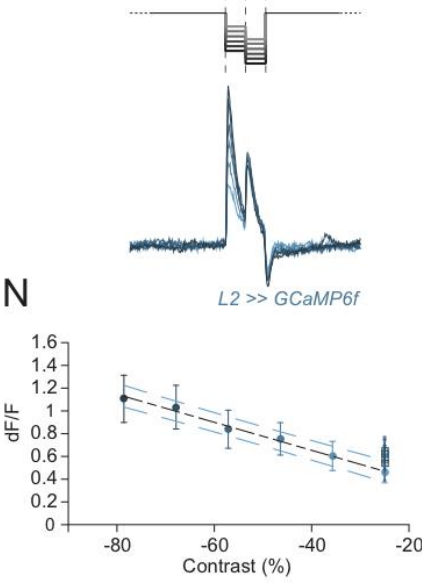

$\mathrm{R}$

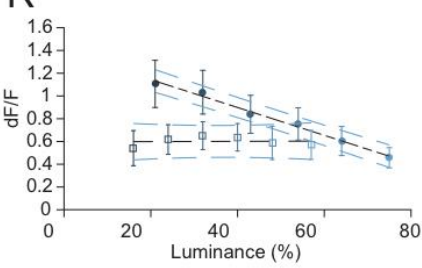

C ND 1.3

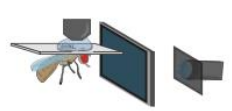

$50 \%$ OFF step
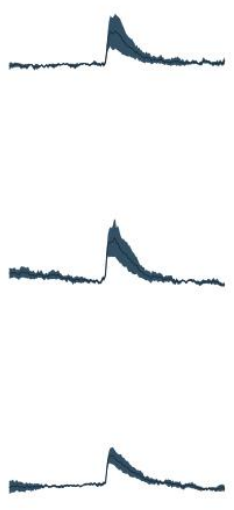

L2 >> GCaMP6f
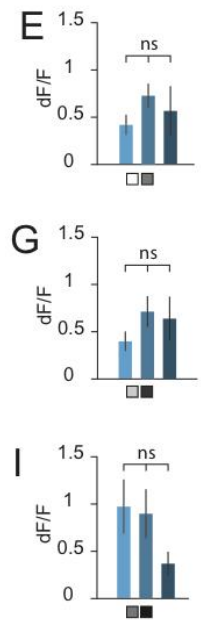

L

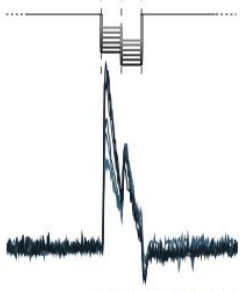

L2 >> GCaMP6f

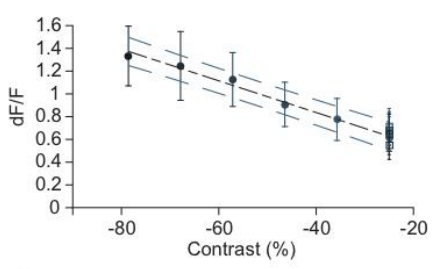

S

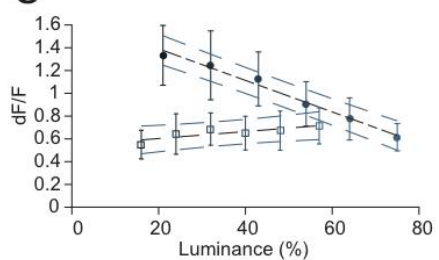

Figure 23. L2 neurons encode contrast relative to the adaptation state.

(A-C) Schematics showing the in vivo two-photon imaging setup. Mean luminance of the visual stimuli, and thereby adaptation state, was shifted using neutral density (ND) filters. This resulted in three different luminance regimes: bright (A), middle (B) and dark (C). (D,F,H) Calcium signals of L2 cells in response to the OFF step ending at gray (D), 50\% OFF (F) and 100\% OFF (H) recorded in three different luminance regimes: no ND (left), ND 0.6 (middle) and ND 1.3 (right). (E,G,I) Bar plots showing the quantification of the L2 peak responses in response to the OFF step ending at gray (E), 50\% OFF (G) and 100\% OFF (I) recorded 
in three different luminance regimes, as indicated by a schematic in (A-C). ANOVA was used to test for differences between $L 2$ responses in three different luminance regimes $(p<0.05)$. $p=0.63$ in $(E), p=0.472$ in (G) and $\mathrm{p}=0.116$ in (I). $\mathrm{N}=4$ flies (32 cells) for $\mathrm{L} 2$ measured in the brightest luminance regime (no ND), $\mathrm{N}=4$ (34) for L2 measured in the middle luminance regime (ND 0.6) and $\mathrm{N}=4$ (33) for L2 measured in the darkest luminance regime (ND 1.2) in (D-I). (J-L) Schematic of the stimulus, consisting of a $30 \mathrm{~s}$ adapting bright period, followed by two sequential OFF steps, each $3 \mathrm{~s}$ long. Average calcium signals of L2 neurons in response to the stimulus shown in (A) in three luminance regimes: the bright $(\mathrm{J})$, middle $(\mathrm{K})$ and the darkest (L). (M-S) Peak calcium responses of L2 neurons plotted as a function of contrast (M-O) or luminance (P-S) measure in three different luminance regimes: bright $(M, P)$, middle $(N, R)$ and dark $(O, S)$. A linear regression model (black dashed line) was fit to the responses to the A step (M-O) or to the A and B steps individually (P-S). The dashed lines indicate the 95\% confidence interval. Responses to the A step are illustrated by circles, responses to the B step by squares. $\mathrm{N}=5$ flies (43 cells) for L2 measured in the brightest luminance regime (no ND), $\mathrm{N}=6$ (48) for L2 measured in the middle luminance regime (ND 0.6) and N = 7 (54) for L2 measured in the darkest luminance regime (ND 1.2) in (D-I). Significance was tested with one-way ANOVA $(\mathrm{p}<0.05) \cdot \mathrm{p}=0.995$ in $(\mathrm{P}), \mathrm{p}=0.992$ in $(\mathrm{R})$ and $\mathrm{p}=0.959$ in $(\mathrm{S})$.

\subsubsection{L3 is required in dim light relative to the adapted range.}

To test whether L3 is encoding absolute luminance or luminance relative to its adapted range, we presented the flies with stimuli while altering the overall mean intensity of the stimulus as described above (Figure 24A-C). We presented the flies with a stimulus that varied randomly between five different light intensities, each lasting $10 \mathrm{~s}$ (as in Figure 21B), and consecutively using different ND filters. L3 calcium responses to the OFF steps in all three light regimes showed a peak response, followed by a plateau response (Figure 24D,F,H) indicating that L3 responds with the same kinetics throughout different luminance regimes. When plotting L3 calcium responses to the same Weber contrast steps, e.g. $100 \%$ ON to grey, $50 \%$ ON to $50 \%$ OFF and grey to $100 \%$ OFF for all three different light regimes (no ND, ND 0.6, or ND1.2), no statistical differences were found between L3 responses (Figure 24D-I). For all three light regimes, L3 was most active in dim light relative to the adapted state (Figure $24 \mathrm{H}$ ).

To test the contrast or luminance-sensitivity of L3 neurons, we presented the flies first with the $30 \mathrm{~s}$ long adapting period, followed by two sequential OFF steps (Figure 24J-L). This stimulus was again shown in three different luminance regimes, using ND filters. As with L2, L3 calcium responses showed the smallest amplitude for all the steps in the brightest regime (Figure 24J) and the biggest amplitude in the darkest luminance regime (Figure 24L). Calcium signals recorded from the L3 axon terminals showed increase in the response amplitude with an increasing contrast step in all regimes, consistent with previous data (Figure 20F-I). Responses to the same 25\% contrast change differed from each other within the individual regimes. Plotting responses as a function of luminance revealed that L3 responses to the A step were similar to the 
responses to the B step at a close by luminance in all three light regimes tested. This argues that L3 neurons are mainly active in dim light relative to the adapted regime.

A

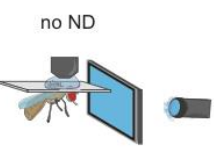

D

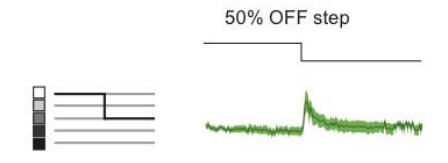

$\mathrm{F}$

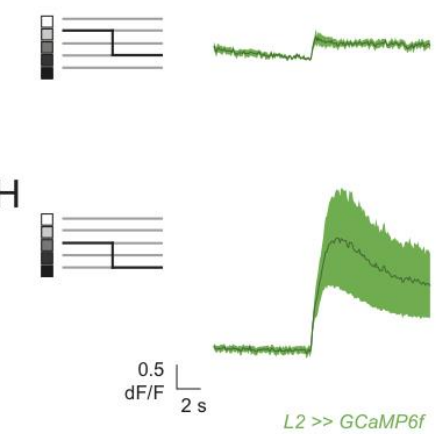

$J$
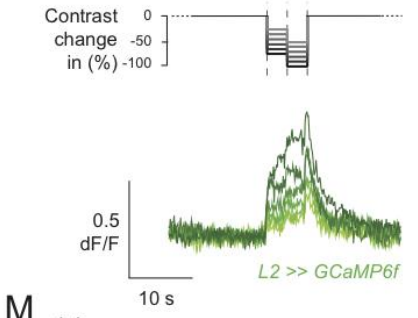

M

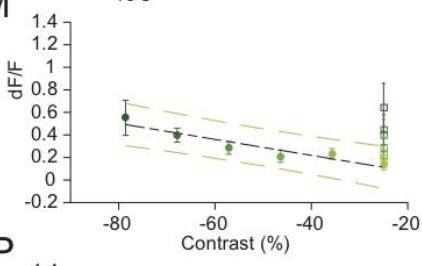

$\mathrm{P}$

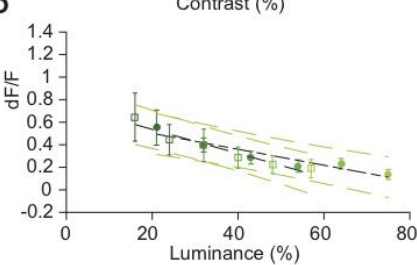

B

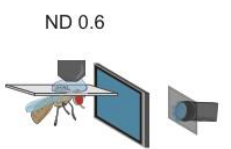

$50 \%$ OFF step
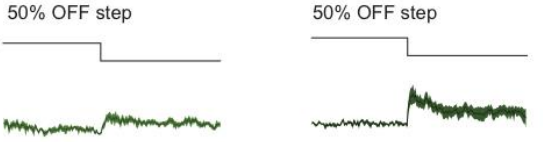

G

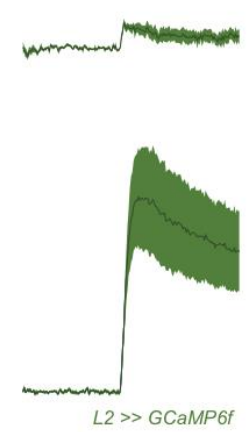

K
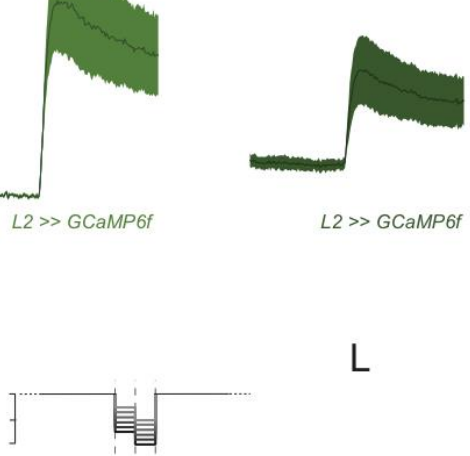

L2 >> GCaMP $6 f$

I

$\mathrm{L}$
E
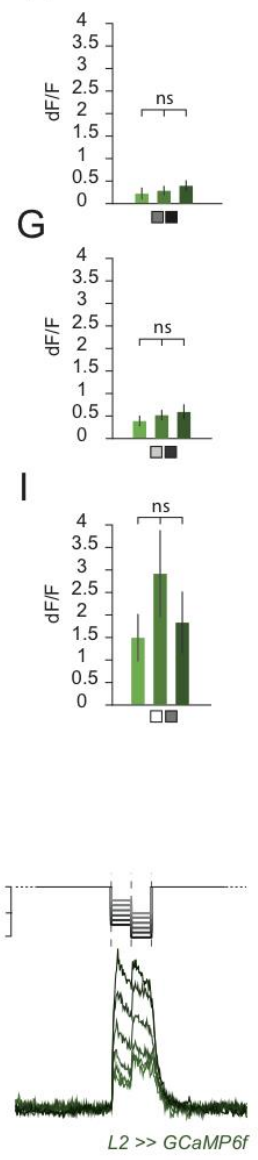

$\mathrm{N}$
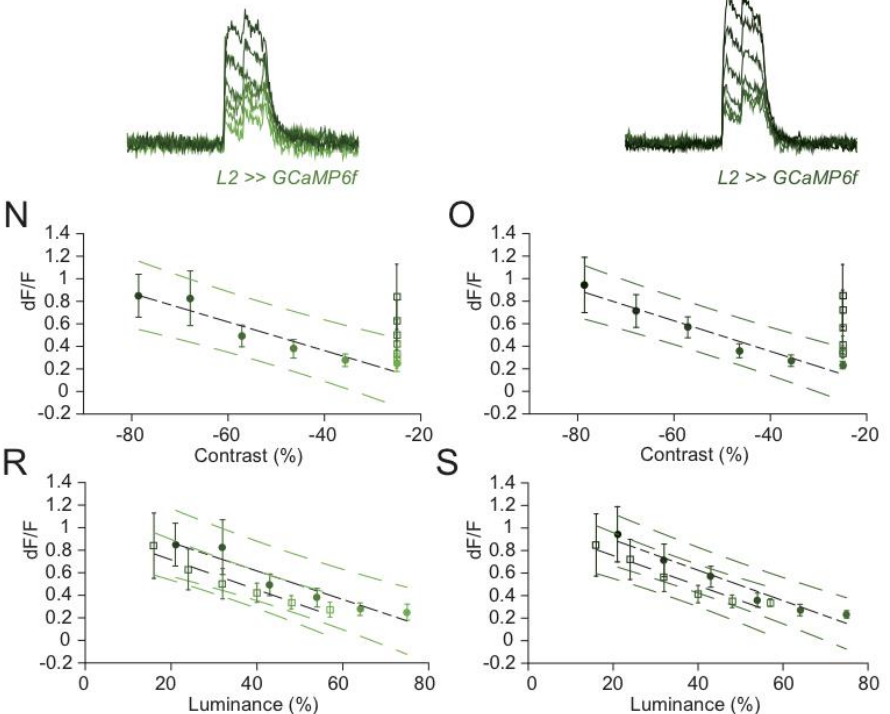

Figure 24. $\mathrm{L3}$ neurons are the most active in dim light relative to the adaptation state.

(A-C) Schematics showing the in vivo two-photon imaging setup. Mean luminance of the visual stimuli, and thereby adaptation state, was shifted using neutral density (ND) filters. (D,F,H) Calcium signals of L3 axon terminals in response to the OFF step ending at gray (D), 50\% OFF (F) and 100\% OFF (H) recorded in three 
different luminance regimes: no ND (left), ND 0.6 (middle) and ND 1.3 (right). (E,G,I) Bar plots showing the quantification of the L3 peak responses in response to the OFF step ending at gray (E), 50\% OFF (G) and $100 \%$ OFF (I) recorded in three different luminance regimes, as indicated by a schematic in (A-C). ANOVA was used to test for differences between L3 responses in three different luminance regimes $(p<0.05)$. $p=$ 0.546 in (E), p = 0.552 in (G), p = 0.405 in (I). $N=5$ (33) for L3 measured in the brightest luminance regime (no ND), $\mathrm{N}=4$ (31) for L3 measured in the middle luminance regime (ND 0.6) and $\mathrm{N}=4$ (38) for L3 measured in the darkest luminance regime (ND 1.2) in (D-I). (J-L) Schematic of the stimulus, consisting of a $30 \mathrm{~s}$ adapting bright period, followed by two sequential OFF steps, each $3 \mathrm{~s}$ long. Average calcium signals of L3 neurons in response to the stimulus shown in (A) in three luminance regimes: the bright (J), middle (K) and the darkest (L). (M-S) Peak calcium responses of L3 neurons plotted as a function of contrast (M-O) or luminance (P-S) measure in three different luminance regimes: bright $(\mathrm{M}, \mathrm{P})$, middle $(\mathrm{N}, \mathrm{R})$ and dark $(\mathrm{O}, \mathrm{S})$. A linear regression model (black dashed line) was fit to the responses to the A step (M-O) or to the A and B steps individually (P-S). The dashed lines indicate the 95\% confidence interval. Responses to the A step are illustrated by circles, responses to the B step by squares. Significance was tested with one-way ANOVA ( $\mathrm{p}<0.05$ ). $\mathrm{p}=0.9104$ in (P), $\mathrm{p}=0.1836$ in (R), $\mathrm{p}=0.0953$ in (S). $\mathrm{N}=5$ flies (28 cells) for L3 measured in the brightest luminance regime (no ND), $\mathrm{N}=6$ (40) for L3 measured in the middle luminance regime (ND 0.6) and $\mathrm{N}=7$ (46) for L3 measured in the darkest luminance regime (ND 1.2) in (D-I).

\subsection{Mechanisms that shape L2 and L3 physiology}

The two LMCs, L2 and L3, are sensitive to fundamentally different visual features. Given that both lamina neurons are first order interneurons downstream of photoreceptors, we next wanted to understand where these different response properties arise. We therefore next probed the potential contribution of different photoreceptors, and tested where in the lamina neuron the distinct properties arise. In addition, we aimed to understand whether L2 and L3 physiological properties are shaped by circuit interactions or as a result of a cell-autonomous mechanism.

\subsubsection{L2 and L3 responses depend on the same major photoreceptor input.}

L2 and L3 are both immediately downstream of photoreceptors and yet their physiological properties are very different: calcium signals in response to light stimuli in L2 axon terminals are transient and contrast-sensitive, whereas calcium responses in L3 axon terminals are sustained and luminance-sensitive. L2 as well as L3 receive major inputs from R1-R6, which are also the main inputs to the motion-detecting circuits (Heisenberg and Buchner, 1977). It has been shown that the inner photoreceptor R8, which is an input to the color pathway and is involved in a color-opponent processing (Schnaitmann et al., 2018), also contributes to motion detection (Wardill et al., 2012). Combining information from these two channels could improve the sensitivity and robustness of the system. Furthermore, several studies suggested that R8 is less adapting than R1-R6 (Hardie, 2011; Hardie et al., 1981; Harris et al., 1976). Thus, we 
wanted to test whether R8 could account for the sustained L3 responses. R8 expresses the blue- (Rh5) and green-sensitive (Rh6) rhodopsins, whereas R1-R6 express Rh1 (Figure 2A-C) (Salcedo et al., 1999). If R8 strongly contributed to the L3 responses, L3 would have a peak response in the wavelengths where rhodopsin Rh5 and Rh6 have their peak sensitivity: the blue-sensitive $\mathrm{Rh} 5$ has a peak at $437 \mathrm{~nm}$ and the greensensitive Rh6 at $508 \mathrm{~nm}$. We therefore tested the spectral efficiency in L2 and L3 neurons using a multispectral synthesizer based on LEDs and a diffraction grating (Figure 25A-D, Belušič et al., 2016). Animals were presented with a light coming from a series of LEDs with peak wavelengths spanning from UV to red light, after the light from these LEDs had been passed through a diffraction grating to a collimating lens and then into the light fiber, which was projected straight into the fly's eye (Figure 25A). Flies were presented with $2 \mathrm{~s}$ long flashes of light, interleaved with $2 \mathrm{~s}$ of darkness, coming from a series of LEDs spanning from UV to red light. To prevent light of different wavelengths emitted by the LED synthesizer reaching the photomultiplier, PMT shutters were closed during the stimulus presentation (Figure 25B). Given that L2 and L3 neurons show an increase in calcium signal to the offset of light, precise timing of the shutter still permitted us to record the full OFF responses of L2 and L3 cells. When we presented sets of light stimuli ranging from UV to red and recorded responses of a single axon terminal, we could observe that neurons responded differently to light coming from different LEDs (Figure 25C). Both L2 and L3 calcium responses recorded from the axon terminals showed the strongest responses in the green part of the visual stimulation. While both L2 and L3 calcium signals showed normal response kinetics, the response amplitude changed in response to the light coming from different LEDs (Figure 25C). Peak responses to each LED presentation were plotted together in one plot, which resulted in a spectral efficiency curve. The spectral efficiency curves of L2 and L3 looked very similar, and both neurons showed peak spectral sensitivities in the cyan (blue and green) part of the spectrum ( $\sim 500 \mathrm{~nm}$, Figure $25 \mathrm{D})$, close to the peak sensitivity of Rh1 (Stavenga et al., 1993). Smaller differences could be observed in the UV part of the spectrum and in L3 spectral efficiency curve we observed a local maximum at around $437 \mathrm{~nm}$, which could come from Rh5-expressing R8s (Figure 25D). Taken together, these results suggest that both L2 and L3 receive predominant input from R1-R6, and argue against a strong contribution from R8 to L3 responses. 
A

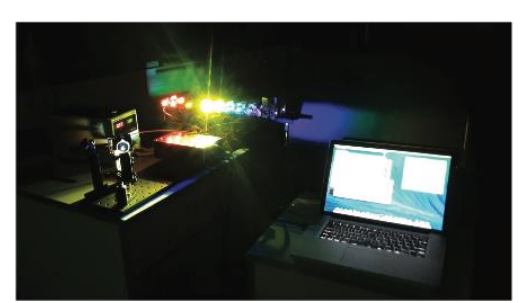

C

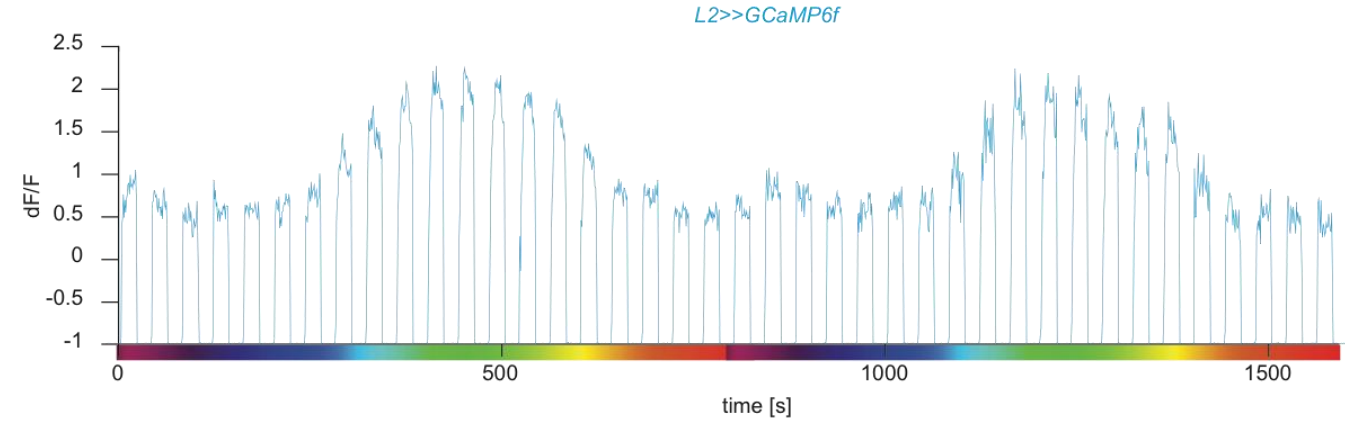

B

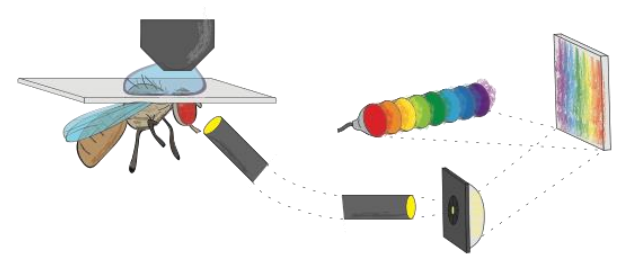

$\mathrm{E}$

L3>>GCaMP $6 f$

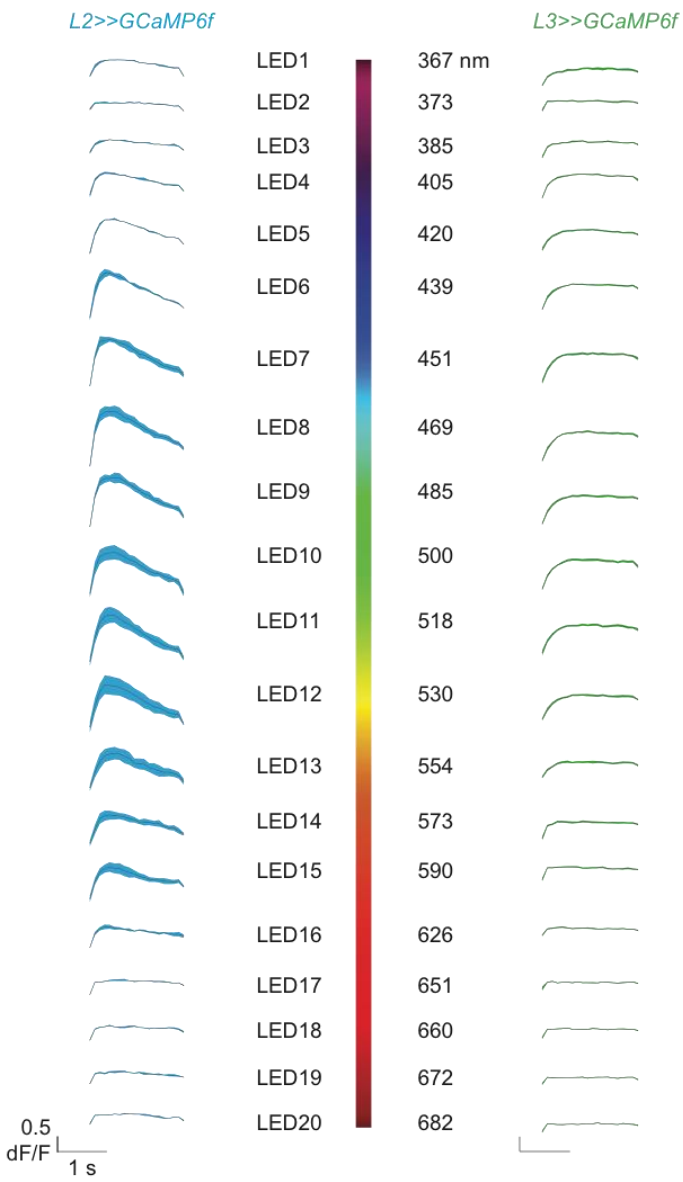

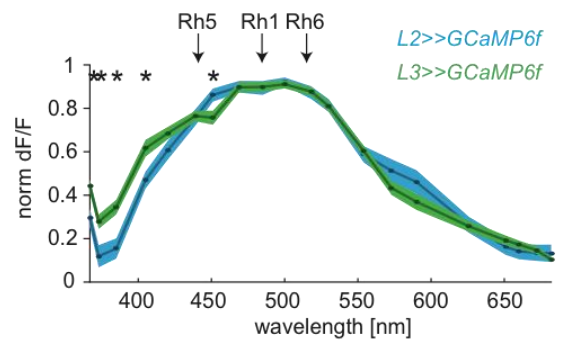

Figure 25. L2 and L3 responses depend on the same photoreceptor input.

(A) Picture of the LedSynth setup. (B) Schematic of the setup depicting the light path. Light coming from 20 different LEDs was projected into a planar reflective diffraction grating, a launching lens and a light guide, pointing at the fly eye. (C) Example calcium trace of a single L2 axon terminal in response to a $2 \mathrm{~s}$ long 
flashes of light coming from a series of LEDs spanning from UV to red light. PMT shutters were closed during the stimulus presentation. 2 sets of 20 LED presentations were presented to the fly. (D) Calcium signals of L2 (blue) and L3 (green) axon terminals in response to the light coming from 20 LEDs spanning from UV to red light. Calcium signals to the offset of light were recorded. (E) Spectral efficiency curves of L2 and L3 neurons. Arrows indicate the peak sensitivities of the R8 rhodopsin Rh5 (437 nm) and Rh6 (508 nm), and the R1-R6 rhodopsin Rh1 (478 $\mathrm{nm}$ ). Traces show L2 (blue) and L3 (green) peak responses to the light offset of different wavelengths. ${ }^{*} \mathrm{p}<0.05$, tested with two-tailed Student $t$ tests. $\mathrm{N}=4$ (17) for $\mathrm{L} 2$ and $\mathrm{N}=8$ (28) for L3 in (D-E).

\subsubsection{Contrast- and luminance-sensitive pathways depend on distinct photoreceptor-to-lamina neuron transformations}

To further test the specific R8 contribution functionally, we genetically eliminated any inputs coming from the outer photoreceptors R1-R6. To do so, we used a ninaE mutant background. ninaE encodes for the major rhodopsin in the fly eye, Rh1 (O'Tousa et al., 1985; Zuker et al., 1985). Therefore, in ninaE mutant background flies, R1-R6 rhodopsin Rh1 is mutated. Thus, only the inner photoreceptors R7 and R8 are functional (Figure 26A). With this, we could test whether R8 is sufficient to provide normal, sustained responses in L3. The ninaE mutant chromosome further carried a mutation in the histamine receptor gene outer rhabdomeres transientless (ort). In invertebrates, histamine is the main neurotransmitter of the photoreceptor cells and post-synaptic to photoreceptors, the large monopolar cells L1-L3 respond to brightness with hyperpolarization, which is chloride-mediated (Hardie, 1989). The histamine gated chloride channel ort is expressed in the lamina neurons and ort mutant flies have significant deficits transmitting photoreceptor signals to the lamina interneurons (Gengs et al., 2002). Cell-type-specific ort expression in L2 or L3 enabled us to restore R7 and R8 photoreceptor inputs selectively onto either L2 or L3, while isolating them from other potential inputs. We first recorded GCaMP6f signals from the axon terminals in control ort, ninaE mutant flies. In these flies, neither L2 nor L3 responded to visual stimuli (Figure 26B). L2 and L3 responses from heterozygous ort, ninaE flies showed normal response amplitude and kinetics. Thus, ort overexpression alone did not change the shape of the L2 or L3 responses. In flies in which an ort transgene was selectively expressed in L2 neurons, L2 showed no visual responses in the mutant background. In contrast, re-expression of ort in L3 led to very weak, transient responses to visual stimulation (Figure 26B). However, these L3 responses did not resemble the normal, persistent activity of L3 neurons. These experiments suggest that R8 alone does not provide major functional input to L3. 
A

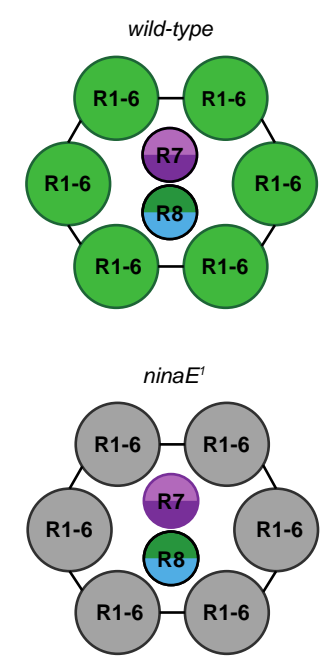

B
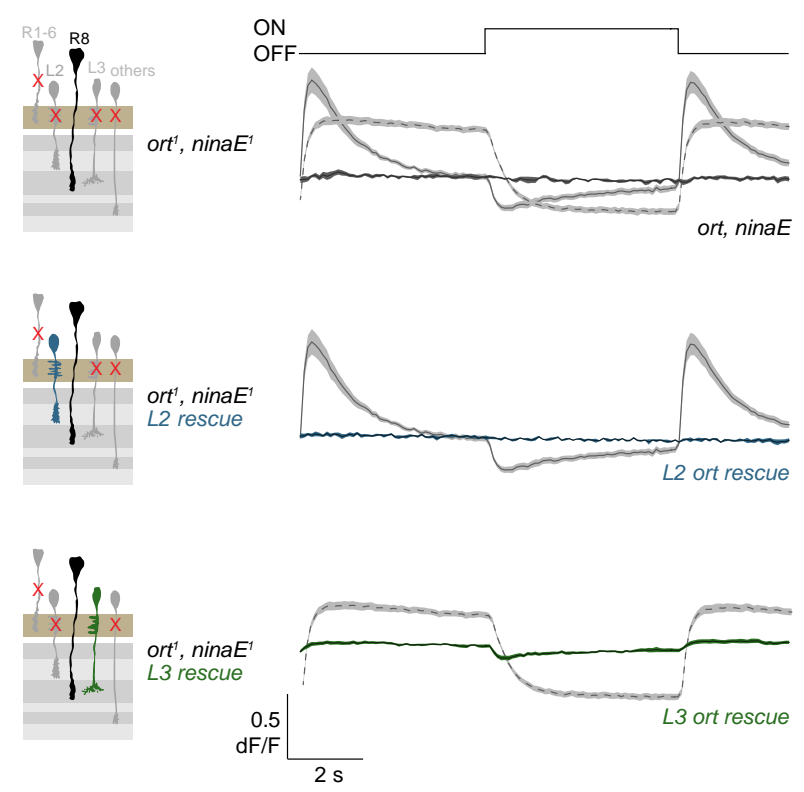

Figure 26. R8 alone does not provide major functional input to L3.

(A) Schematic of $\mathrm{R}$ cell arrangements in one ommatidium. Cross-section perspective. R7 and R8 rhabdomeres are shown in the same plane for ease of representation. Top: wild-type ommatidium where all $\mathrm{R}$ cells are functional. Bottom: schematic showing a ninaE mutant ommatidium. The only functional R cells are R7 and R8. Modified from (Jackowska et al., 2007). (B) ort rescue experiments in a ninaE mutant background. Schematics illustrate this experiment for an ninaE, ort mutants, L2 ort rescue and L3 ort rescue. Flies receive no inputs from R1-R6, and only a single photoreceptor to lamina neuron synapse is intact due to re-expression of the ort gene. Average calcium signals (dF/F) of L2 or L3 in response to $5 \mathrm{~s}$ full-field flashes. There are no visual responses in a ninaE, ort mutant background (dark gray). Heterozygous ninaE1, ort $^{1}$ control responses show normal response amplitude and kinetics, shown in light gray for L2 (continuous line) and L3 (dashed line). In L2 ort rescue flies (blue, middle), there are no visual responses. In L3 ort rescue flies (green, bottom), there are very small visual responses to light flashes. $\mathrm{N}=14$ (95) for L2 and $\mathrm{N}=$ 29 (198) for L3 ninaE ${ }^{1}$, ort ${ }^{1}$ heterozygous controls; $\mathrm{N}=4$ (59) for ninaE $E^{1}$, ort ${ }^{1}$ mutants; $\mathrm{N}=3$ (33) for L2 ort rescues; and $\mathrm{N}=10$ (142) for L3 ort rescues.

The experiments described above suggest that L2 and L3 properties derive from the same major R1-R6 photoreceptor input. However, it has been shown that ninaE mutant flies show structural deterioration of both inner and outer photoreceptors. The degeneration of the photoreceptors is gradual and can take a couple of weeks (Leonard et al., 1992). Because flies imaged here were only a few days old, the degeneration was most likely rather small. Despite this, we wanted to test the potential different photoreceptor contribution to L2 and L3 using another approach. To do so, we performed a so-called norpA rescue experiment. The norpA gene encodes a phosphatidylinositol-specific phospholipase $\mathrm{C}$ (PLC) that is essential for phototransduction (Yoshioka et al., 1985). Mutations in norpA abolish the photoreceptor potential by eliminating PLC activity in the fly eye, making the fly blind (Hotta and 
Benzer, 2006; Pak et al., 1970). Using Gal4 drivers, it is possible to functionally rescue the phototransduction in individual photoreceptor types by re-expressing norpA. Using different rhodopsin drivers, Rh1-Gal4 expressing in R1-6 or panR8-Gal4 expressing in all R8 photoreceptors, allowed us to specifically test the contribution of different photoreceptors onto L2 and L3. This allowed us to test whether different photoreceptor classes are sufficient to drive L2 or L3 responses. We first tested whether R1-R6 are sufficient to drive L2 responses. To do so, we rescued norpA in R1-R6, using an Rh1-Gal4 line (Figure 27A). We then simultaneously recorded visually evoked signals in L2, by expressing GCaMP6f in L2 using a different binary expression system, the LexA-lexAop system. When we measured L2 calcium signals in a norpA mutant control background, these neurons did not respond to visual stimulation, confirming that these flies were blind (Figure 27B). When we rescued norpA in R1-R6, and recorded from L2 neurons, L2 neurons showed indistinguishable responses compared to Gal4 control (Figure 27B). We also tested L2 responses in R1-R6 rescued animals in response to ON and OFF flashes from an intermediate gray background and did not observe any difference compared to control L2 neuronal responses. In both cases L2 neurons showed increase in their calcium signal to the OFF and decrease to the light onset (Figure 27C). Furthermore, response kinetics of L2 neurons were not changed in R1-R6 rescued animals. Analyzing peak responses to the two OFF steps, grey-to-OFF and ON-to-grey, revealed that control L2 responses and L2 responses in R1-R6 rescued animals showed non-significant change in response amplitude to the same Weber contrast (Figure 26D). This shows that R1-6 photoreceptors are sufficient to drive appropriate L2 responses. While L2 ort rescue experiments in a ninaE mutant background suggested that L2 do not receive any functional input from $\mathrm{R} 8$, we decided to test in a complementary experiment whether R8s are sufficient to drive L2 responses. To do so, we expressed norpA specifically in R8 cells (Figure 27E). When we recorded L2 GCaMP6f signals in control norpA mutant flies, L2 did not respond to visual stimulation (Figure 27F). Upon selective expression of norpA in R8 neurons, L2 neurons responded to visual stimuli, albeit with much smaller amplitude than control L2 neurons only expressing the R8-specific Gal4 (Figure 27F). Response amplitude of L2 neurons in R8 rescued animals was even smaller when flies were presented with $\mathrm{ON}$ and $\mathrm{OFF}$ flashes from an intermediate grey (Figure 27G). Peak responses of control L2 responses and L2 responses in R8 rescued animals to the two OFF steps, grey-to-OFF and ON-to-grey, showed a significant change in their response amplitude (Figure $27 \mathrm{H}$ ). This suggests that $\mathrm{L} 2$ receives an indirect input from R8. The result is in slight contrast to the result from ninaE mutants described above. The difference might be due to some photodegeneration in ninaE mutants, or due 
to a stronger R8-photoreceptor input after norpA overexpression. However, all result agree that the predominant input into L2 is coming from R1-R6. Taken together, while R8 might provide some indirect input to L2 neurons, only outer photoreceptors R1-6 seem to be sufficient to restore normal L2 responses.

A

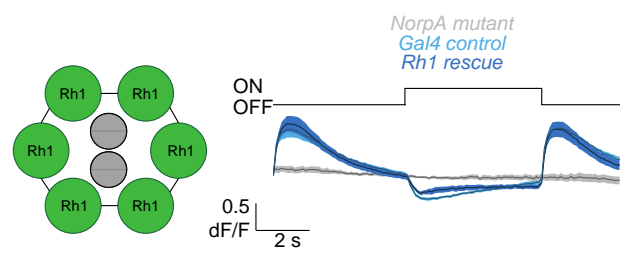

$E$

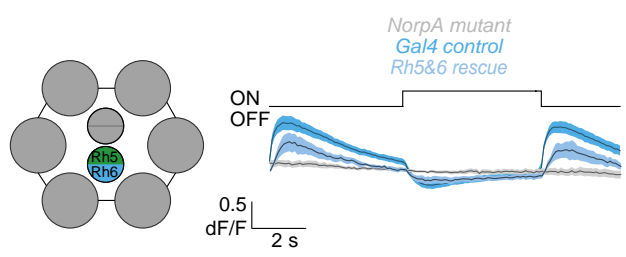

C

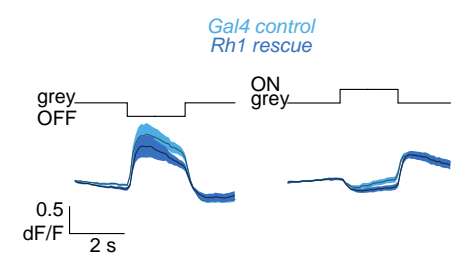

G

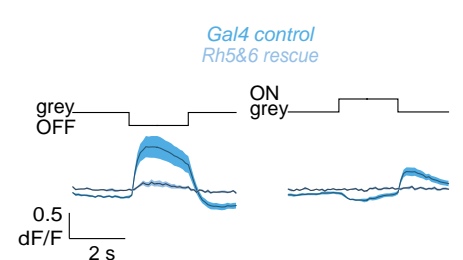

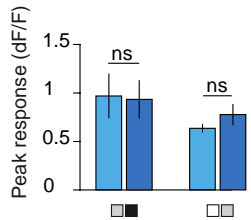

D

$\mathrm{H}$

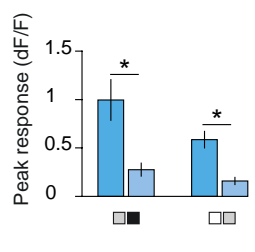

Figure 27. Outer photoreceptors R1-6 are sufficient to restore normal L2 responses.

(A) Schematic of one ommatidium depicting the norpA rescue experiment in which norpA was rescued in R1-6. (B-D) R1-R6 norpA rescue experiment, recorded from L2 neurons. L2 responses imaged in norpA mutant background are shown in gray, Gal4 L2 control responses are shown in light blue and L2 responses in an R1-R6 rescued animal are shown in dark blue. (B) Calcium signals recorded from L2 to $5 \mathrm{~s}$ full-field flashes. $\mathrm{N}=6$ (32) for norpA mutant control flies, $\mathrm{N}=5$ (43) for Gal4 L2 control flies and $\mathrm{N}=12$ (94) for L2 responses in an R1-R6 rescued animals. (C) Calcium signals measured from L2 controls and L2 in R1-R6 rescued animals in response to ON and OFF flashes from a gray background. (D) Bar plots showing the quantification of the peak responses of L2 control and L2 in R1-R6 rescued animals to the two OFF steps shown in (C): ON-to-gray and gray-to-OFF. ${ }^{*}$ p $<0.05$, tested with two-tailed Student $t$ tests. $\mathrm{N}=5$ (45) for L2 controls and $\mathrm{N}=10$ (73) for L2 in R1-R6 rescued animals in (C-D). (E) Schematic of one ommatidium depicting the norpA rescue experiment in which norpA was rescued in R8. (F-H) R8 rescue experiment, recorded from L2 neurons. L2 responses imaged in norpA mutant background are shown in gray, Gal4 L2 control responses are shown in light blue and L2 responses in an R8 rescued animals are shown in dark blue. (F) Calcium signals recorded from L2 to periodic $5 \mathrm{~s}$ full-field flashes. $\mathrm{N}=6$ (32) for norpA mutant control flies, $\mathrm{N}=7$ (49) for Gal4 L2 control flies and $\mathrm{N}=8$ (23) for L2 responses in R8 rescued animals. (G) Calcium signals measured from L2 controls and L2 in R8 rescued animals in response to ON and OFF flashes from a gray background. (H) Bar plots showing the quantification of the peak responses of $\mathrm{L} 2$ control and $\mathrm{L} 2$ in R8 rescued animals to the two OFF steps shown in (G): ON-to-gray and gray-to-OFF. ${ }^{*} \mathrm{p}<0.05$, tested with two-tailed Student $t$ tests. N = 7 (56) for L2 controls and N = 9 (50) for L2 in R8 rescued animals in (G-H).

To see whether different photoreceptor classes drive L3 responses, we performed the same set of experiments as described above, only that this time we recorded calcium signals from L3 neurons (Figure 28A-H). We first tested whether R1-R6 are sufficient to drive L3 responses. L3 neurons in a norpA mutant background did not respond to visual 
stimulation, confirming that these flies were blind (Figure 28B). To rescue phototransduction in outer photoreceptors we rescued norpA in R1-6 (Figure 28A) and recorded calcium responses to flashes in L3 neurons. L3 calcium responses in R1-R6 rescued animals were indistinguishable from Gal4 control L3 responses (Figure 28B). We then tested L3 responses in R1-R6 rescued animals in response to ON and OFF flashes from gray background. Responses looked similar between L3 in R1-R6 rescued animals and controls to the two OFF steps. Comparing responses to the two OFF steps showed that L3 neurons in R1-R6 rescued animals responded with high amplitude to the grey-to-OFF step, but barely responded to the ON-to-grey step, just as the control L3 neurons (Figure 28C-D). Furthermore, response amplitude of control L3 and L3 in R1R6 rescued animals was not changed. Thus, R1-6 photoreceptors are sufficient to drive normal L3 responses.

We then tested whether the inner R8 photoreceptors are also sufficient to drive L3 responses. Thus, we rescued the function of R8 (Figure 28E) by expressing norpA in R8 cells. Expression of norpA in R8 rescued some visual responses in L3 neurons, although the responses were weak (Figure 28F). L3 neurons in R8 rescued animals practically did not respond to $\mathrm{ON}$ and $\mathrm{OFF}$ flashes from intermediate grey, as also shown when quantifying the peak response amplitude (Figure 28G-H). Together, while R8 does provide an input to L3, its contribution is rather small, and dependent on stimulus context.

To conclude, these data show that L2 and L3 properties derive from the same major R1R6 photoreceptor input.
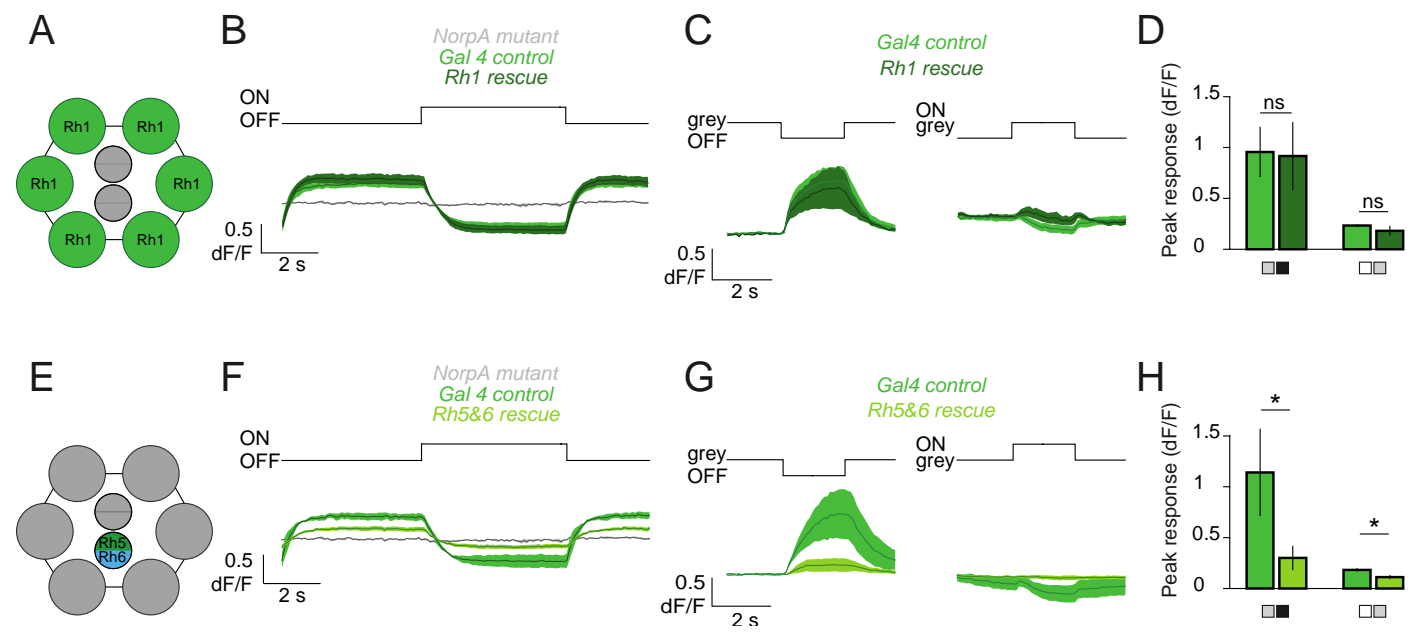

Figure 28. Outer photoreceptors R1-6 are sufficient to restore normal L3 responses.

(A) Schematic of one ommatidium depicting the norpA rescue experiment in which norpA was rescued in R1-6. (B-D) R1-R6 norpA rescue experiment, recorded from L3 neurons. L3 responses imaged in norpA mutant background are shown in gray, Gal4 L3 control responses are shown in light green and L3 responses in an R1-R6 rescued animal are shown in dark green. (B) Calcium signals recorded from L3 to 5 s full-field 
flashes. . N = 6 (21) for norpA mutant control flies, N = 6 (45) for Gal4 L3 control flies and N = 6 (44) for L3 responses in an R1-R6 rescued animals. (C) Calcium signals measured from L3 controls and L3 in R1-R6 rescued animals in response to $\mathrm{ON}$ and $\mathrm{OFF}$ flashes from a gray background. (D) Bar plots showing the quantification of the peak responses of L3 control and L3 in R1-R6 rescued animals to the two OFF steps shown in (C): ON-to-gray and gray-to-OFF. ${ }^{*} \mathrm{p}<0.05$, tested with two-tailed Student $t$ tests. $\mathrm{N}=6$ (50) for L3 controls and N = 5 (43) for L3 in R1-R6 rescued animals in (C-D). (E) Schematic of one ommatidium depicting the norpA rescue experiment in which norpA was rescued in R8. (F-H) norpA rescue in R8, recorded from L3 neurons. L3 responses imaged in norpA mutant background are shown in gray, Gal4 L3 control responses are shown in light blue and L3 responses in a R8 rescued animals are shown in dark blue. (F) Calcium signals recorded from L3 to periodic $5 \mathrm{~s}$ full-field flashes. $\mathrm{N}=3$ (21) for norpA mutant control flies, $N=6$ (21) for Gal4 L3 control flies and N = 15 (46) for L3 responses in R8 rescued animals. (G) Calcium signals measured from L3 controls and L3 in R8 rescued animals in response to ON and OFF flashes from a gray background. $(\mathrm{H})$ Bar plots showing the quantification of the peak responses of L3 control and L3 in R8 rescued animals to the two OFF steps shown in (G): ON-to-gray and gray-to-OFF. ${ }^{*} \mathrm{p}<0.05$, tested with twotailed Student $t$ tests. N = 4 (28) for L3 controls and N = 16 (129 cells) for L3 in R8 rescued animals in (G-H).

\subsubsection{R1-R6 photoreceptors show sustained responses to flashes}

To determine how the photoreceptor inputs to L2 and L3 respond under our stimulation protocol and imaging conditions, we wanted to measure calcium responses in R1-R6 axon terminals. Using the Rh1-Gal driver line, we expressed GCaMP6f specifically in outer photoreceptors R1-R6. We then wanted to record calcium signals in response to light flashes in the axon terminals using in vivo 2-photon imaging. Despite strong GCaMP6f expression in R1-R6, we did not notice any calcium responses to visual stimuli (data not shown). While axon terminals of L2 and L3 project into the medulla region and allow for easy optical access, R1-R6 terminals are located in the lamina. Since the lamina is located just underneath the retina and is covered with the cornea, imaging photoreceptor responses is technically challenging. Rotating the fly head and removing the medulla in order to expose the lamina did not result in visible R1-R6 responses. However, Asteriti et al., (2017) published a paper stating that Gal4-mediated expression caused significant abnormalities in photoreceptor structure and physiology. The authors achieved to measure calcium responses in R1-R6 axon terminals using a direct ninaEGCaMP6f fusion (Asteriti et al., 2017). Calcium signals recorded from R1-R6 cells captured the steady-state component of the photoreceptor response (Figure 29A), which increased at the onset of light and decreased at the offset, consistent with invertebrate photoreceptors depolarizing to light (Fain et al., 2010; Hardie and Postma, 2008). When we presented ninaE-GCaMP6f flies with OFF and ON flashes from intermediate grey, calcium signals recorded from R1-R6 responded with a decrease in calcium signal to the OFF flash and with an increase to the ON flash and the amplitude of the response to the two OFF steps, gray-to-OFF and ON-to-gray, was the same (Figure 
29B). Furthermore, R1-R6 calcium signals in response to long light flashes were sustained, however a slight decrease in the signal during the presentation of $60 \mathrm{~s}$ long flashes could be observed (Figure 29C), which might indicate a slow photoreceptor adaptation. When flies were presented with flashes of random intensities, photoreceptors followed the increase or decrease of intensities in an almost linear manner within the stimulus regime tested (Figure 29D,E). Thus, in contrast to L3, photoreceptors did not display the nonlinear dark-sensitivity of L3. To explicitly test contrast versus luminance sensitivity in R1-R6, we adapted flies to a bright background and then provided two different consecutive OFF steps, of which the second step was always at 25\% contrast but varied in luminance (see Figure 20A, Figure 29F-H). R1-R6 calcium responses to the various $25 \%$ step stimuli differed and their responses scaled as a function of luminance (Figure 29G,H). In other words, peak calcium responses to the A step had similar amplitude to the peak responses to the B step at a close by luminance. In conclusion, the calcium signal in photoreceptors still carries information about luminance. L2 and L3 receive the same major sustained input from R1-R6 and they transform the input into two different signals: L2 is derivative taking, it amplifies the peak photoreceptor responses and reports contrast changes, whereas L3 enhances responses to dim light and reports luminance. 
A
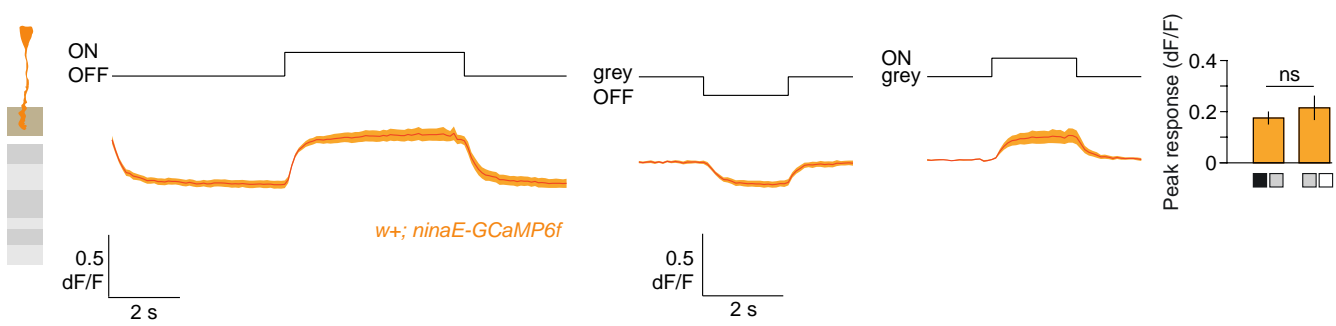

C

ON

0.5
$\mathrm{dF} / \mathrm{F}$

$10 \mathrm{~s}$

$\mathrm{D}$

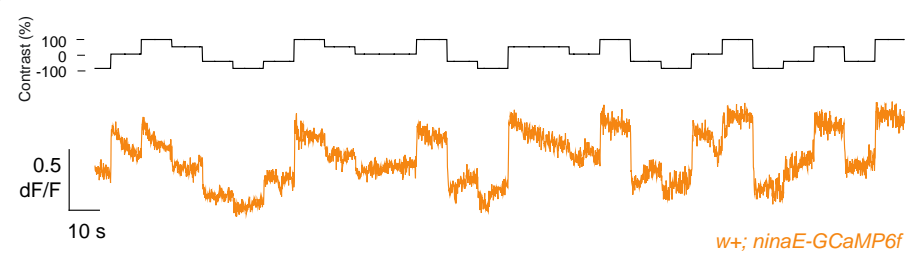

E

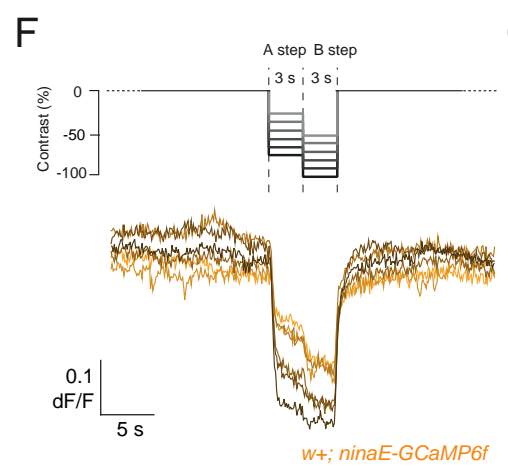

G

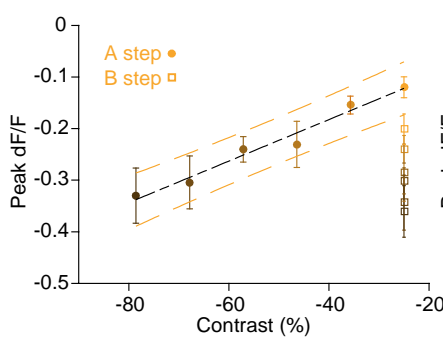

$\mathrm{H}$
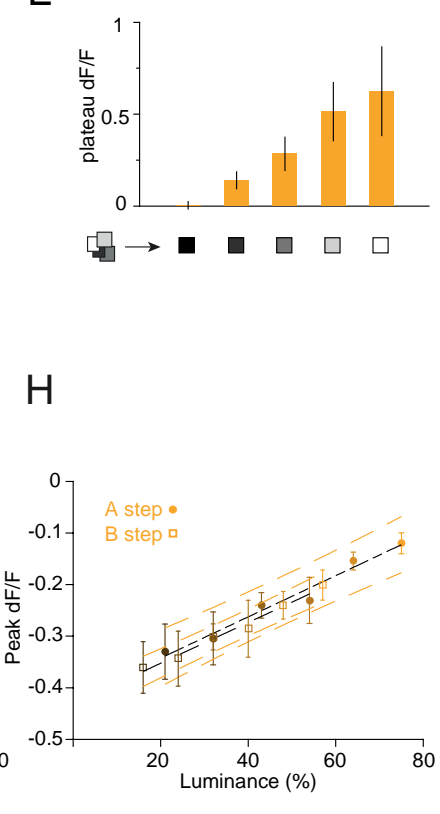

Figure 29. R1-R6 calcium signals show sustained responses to flashes.

(A) Average calcium signals of R1-R6 terminals in ninaE-GCaMP6f flies to $5 \mathrm{~s}$ full-field flashes. $\mathrm{N}=8$ (73). (B) Average calcium responses of R1-6 in ninaE-GCaMP6f flies to OFF (left) or ON (right) flashes from the intermediate gray. Sample sizes are N = 7 (64). (C) Average calcium signals (dF/F) of R1-R6 to $60 \mathrm{~s}$ full-field flashes. Sample sizes are N $=5$ (50). (D) Calcium signal of a single ROI recorded from an R1-R6 axon terminal in response to $10 \mathrm{~s}$ light flashes of random intensities. (E) Bar plots showing the plateau calcium response of R1-R6 axon terminal across different luminance, pooled across all steps. Sample size is N = 10 (89). (F) Schematic of the stimulus, consisting of an adapting bright period, followed by two sequential OFF steps. Average calcium signals of R1-R6 are shown. Darker traces correspond to OFF steps of larger amplitude. (G-H) Peak calcium signals of R1-R6 cells plotted as function of contrast (G) or luminance (H). Responses to the A step are illustrated by circles, responses to the B step by squares. A linear regression model (black dashed line) was fit to the responses to the A step in (G) or to the A and B steps individually (H). The dashed lines indicate a 95\% confidence interval. $\mathrm{N}=7$ (61). 


\subsubsection{L2 amplifies contrast information and discards luminance information from photoreceptors in a circuit-dependent manner}

We next wanted to determine where and how these distinct photoreceptor-to-lamina neuron transformations occur. We first checked where within the neuron L2 enhances its contrast response and discards luminance information. To do so, we recorded calcium responses from different neuronal regions: its dendrites, along the axons and in the axon terminals (Figure 30A). The response amplitude measured in dendrites was very small and this data set was very difficult to obtain. Since the dendrites of L2 are located in the lamina region, recording from them was more difficult due to the cornea and medulla covering the lamina. Thus, we only observed visible responses in dendrites in less than $50 \%$ of the flies tested, compared to almost $100 \%$ when imaging from L2 and L3 axon terminals. Responses from the L2 dendrites were small, reaching maximum $10-20 \% \mathrm{dF} / \mathrm{F}$ (Figure 30A). The response amplitude increased along the neuron and was much more pronounced in the axon terminals where it reached $100 \% \mathrm{dF} / \mathrm{F}$. Furthermore, L2 responses to full-field flashes were more sustained in the dendrites than in the axon terminals (Figure 30A). However, during the 10 second presentation of the stimulus, L2 responses returned to the (almost) same baseline in all three regions (Figure 30B). Taken together, the amplification of a weak postsynaptic transient component and the elimination of the sustained component appear to occur after the photoreceptor-to-L2 synapse along the L2 neuron.

A

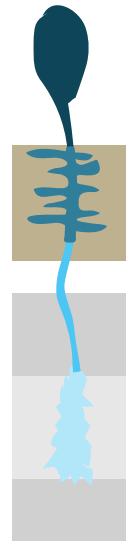

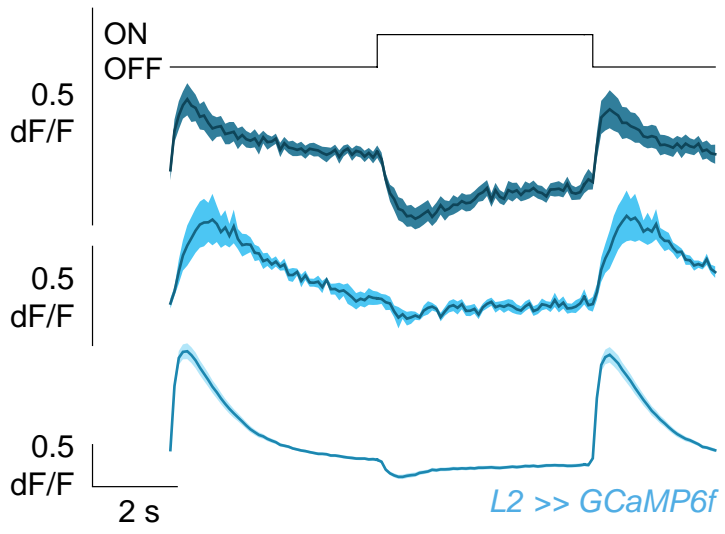

B

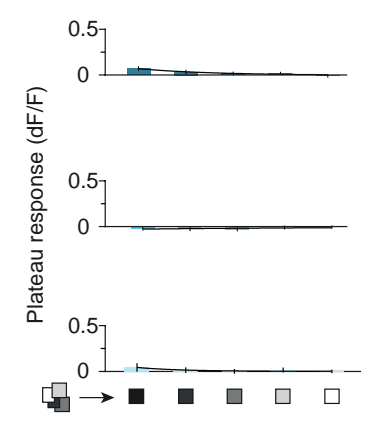

Figure 30. $L 2$ response amplitude increased along the $L 2$ neuron.

(A) Average calcium signals (dF/F) recorded from different regions of L2 neurons: dendrites (dark blue), axons (blue) and axon terminals (bright blue). $\mathrm{N}=9$ (34) for L2 dendrites, $\mathrm{N}=7$ (23) for L2 axons and $\mathrm{N}=$ 23 (130) for L2 axon terminals. (B) Bar plots showing the plateau calcium response of L2 neurons recorded from different regions: dendrites, axons and axon terminals. $N=8$ (25) for L2 dendrites, $N=7$ (19) for L2 axons and $\mathrm{N}=16$ (436) for L2 axon terminals. All traces and bars show mean \pm SEM. 
This signal transformation within the L2 neuron could either be shaped by cellautonomous mechanisms or by circuit interaction. To test the hypothesis that the L2 responses are circuit dependent, we genetically isolated L2 from all circuit interactions by ort rescue. As described above (Figure 25), the ort mutant was thought to abolish all photoreceptor to lamina neuron communication (Gengs et al., 2002; Rister et al., 2007). To create ort mutants, we used a defined null allele containing a 569-bp deletion of the ort gene, which leads to a premature Stop codon in trans to a deficiency deleting the entire ort locus (ort $^{1} / \operatorname{ort}^{D f}$ ) (Figure 31A-B).

A

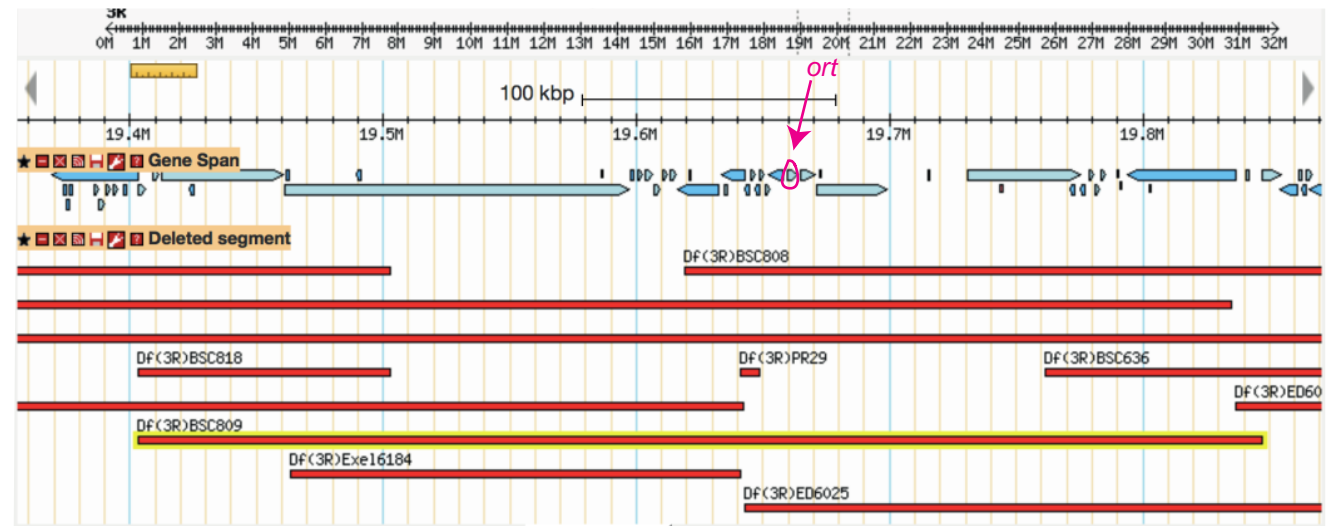

B

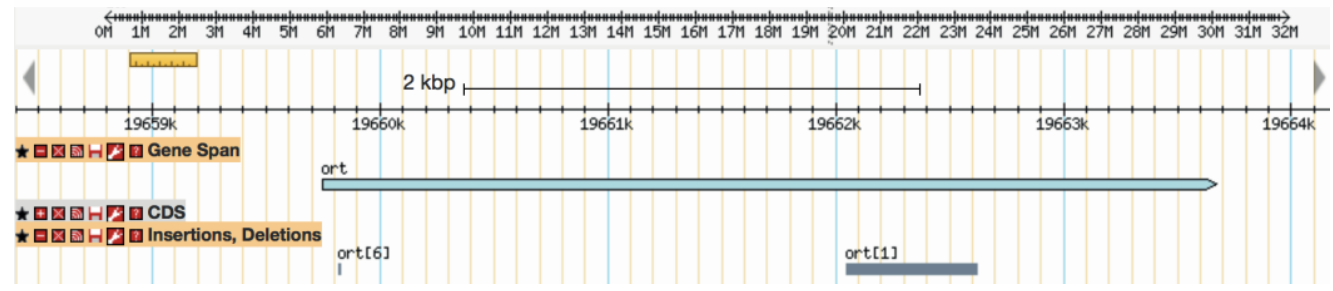

Figure 31. Df(3R)BSC809 deletes the entire ort locus and ort $^{1}{ }^{1}$ is an ort null allele.

(A) A snapshot of GBrowse (www.flybase.org) in the region 3R: 19357748 .. 3R: 19865672 . ort gene region is shown in the 'Gene Span' track at the top as blue arrows and is marked by an arrow. The ort gene sequence location is 3R:19 659748 .. 3R: 19663 672. Yellow colored Df(3R)BSC809 in the 'Deleted segment' track is the deficiency used in the ort experiment. Df(3R)BSC809 deletes 3R: 19403413 .. 3R: 198 470 010. Note that the deficiency deletes the entire ort locus. (B) A snapshot of GBrowse in the region 3R: 19658793 .. 19663 990. ort gene region is shown in the 'Gene Spa' track at the top as blue arrows. In the 'Insertions, Deletions' track ort1 deletion is the deletion affecting the ort gene. It deletes 3R: 19662047 .. 19 662616.

Using cell-type specific ort expression in L2, we specifically restored the photoreceptorto-L2 connection (Figure 32A). When we presented the flies with $100 \%$ full-field flashes and recorded from L2 in an ort mutant background, L2 neurons showed a small but nonzero response to light flashes (Figure 32A). This was unexpected, because previous 
reports have reported that ort mutants are motion blind (Joesch et al., 2010; Rister et al., 2007). Despite this controversial result, we are confident with our result, because we, for the first time, used a defined null allele of the ort gene (Figure 31A-B). Thus, our results argue in favor of ort-independent phototransmission, making the other histamine-gated $\mathrm{Cl}^{-}$channel gene hisCl1 the strongest candidate (Gengs et al., 2002; Schnaitmann et al., 2018). When we re-expressed ort in an ort mutant background (ort ${ }^{1} / \operatorname{ort}^{D f}$ ), L2 responses increased in amplitude (Figure 32A). The initial period of the flash response recorded from these L2 ort rescue neurons did not differ from heterozygous controls. Interestingly, responses in an L2 ort rescue fly appeared to display a more sustained plateau response than heterozygous controls (Figure 32A). To test whether this argues for a luminance-sensitive component, we next recorded calcium signals from heterozygous control and L2 ort rescue flies in response to flashes of different intensities (Figure 32B). As expected, heterozygous L2 control axon terminals showed a normal, transient response to all intensity steps that returned to one common baseline. This could already be seen in the responses of single axon terminal (Figure 32B). In contrast, L2 ort rescue neurons displayed a plateau response in addition to its normal peak response. This plateau response depended on the intensity of the stimulus (Figure 32B). Quantification in many cells and across many flies by pooling these plateau responses of L2 ort rescue neurons for all steps ending at a given luminance confirmed that the calcium signal did not return to baseline. In addition, the plateau response inversely correlated with the stimulus intensity and showed a nonlinear preference for dim stimuli (Figure 32C). This suggests that L2 ort rescue neurons are not purely contrast-sensitive, indicating that elimination of the luminancesensitive baseline in L2 neurons is circuit dependent. 
A
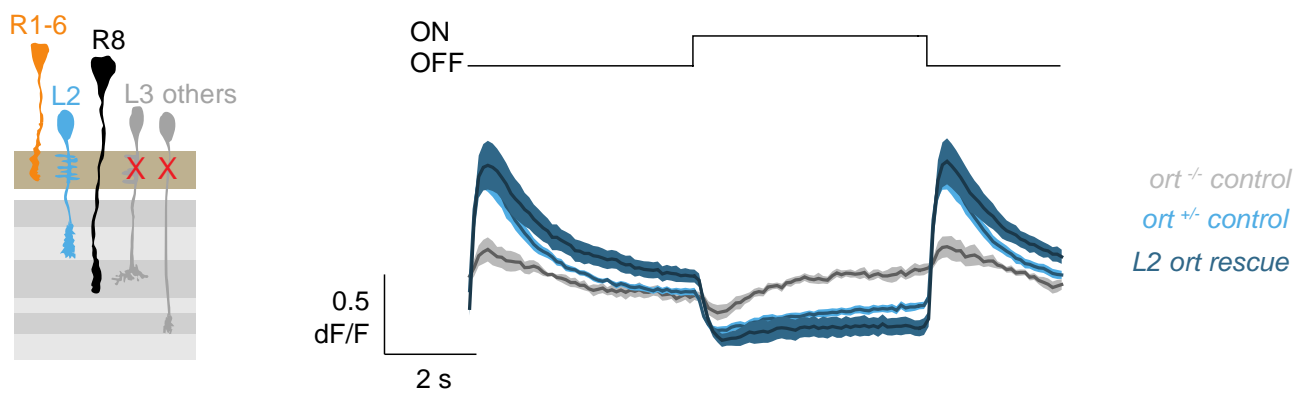

B

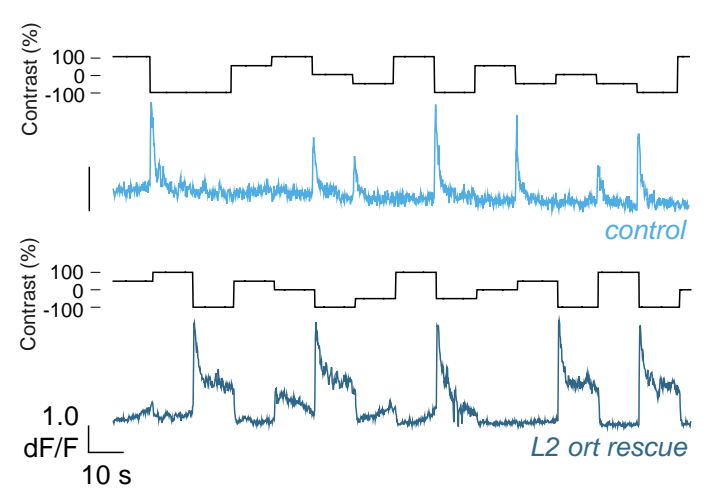

C

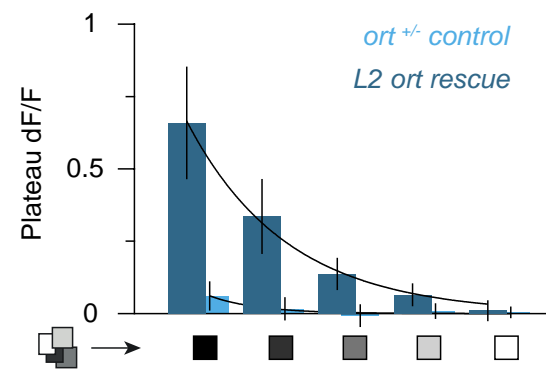

Figure 32. L2 discards luminance information from photoreceptors in a circuit-dependent manner.

(A-C) L2 ort rescue experiment. ort null ( $\left.\operatorname{ort}^{1} / \operatorname{ort}^{D f}\right)$ mutants are shown in light gray. Heterozygous L2 control responses are shown in bright blue. In an L2 ort rescue (dark blue), L2 is genetically isolated from its circuit environment, and is the only lamina neuron receiving functional input from photoreceptors. (A) Average calcium signals of L2 terminals to $5 \mathrm{~s}$ full-field flashes. In an ort null mutant background, L2 responds minimally to light flashes. L2 responses in an L2 ort rescue are similar to heterozygous controls (light blue), with a more pronounced sustained component. $\mathrm{N}=6$ (26) for ort null mutant control flies, $\mathrm{N}=$ 14 (83) for heterozygous ort control and $\mathrm{N}=9$ (74) for L2 ort rescue. (B) Calcium signals recorded from single L2 axon terminals. L2 controls show transient responses to intensity changes that return to one baseline. In an L2 ort rescue, L2 responses are transient, but do not return to one common baseline, and contain a sustained plateau component. (C) Bar plots showing the plateau calcium response of genetically isolated (dark blue) and control (light blue) L2 neurons at different luminance. $\mathrm{N}=16$ (140) for heterozygous ort control and N = 13 (143) for L2 ort rescue.

To test whether the peak L2 response were still contrast-sensitive, we adapted flies to a bright background and then provided two different consecutive OFF steps, of which the second step was always at $25 \%$ contrast but varied in luminance (see Figure 17A). The two OFF steps, A and the B step, were both $10 \mathrm{~s}$ long. This allowed L2 control neurons to return to baseline. We first checked whether L2 peak responses are contrast-sensitive. From average calcium signals of L2 control and L2 ort rescue neurons we observed that L2 control neurons returned to one baseline as expected, but L2 ort rescue neurons did 
not (Figure 33A). However, when plotting peak responses of L2 ort rescue neurons, their responses did not differ from control L2 neurons (Figure 33A-E). Responses to the different $25 \%$ contrast steps of both control and ort rescue neurons were indistinguishable from each other. Thus, genetically isolated L2 neurons still carried contrast information in their initial response. We then analyzed the plateau component of the response (Figure 33F-J). L2 control flies returned to the same baseline, regardless of the contrast and luminance (Figure 33F-H). In contrast, plateau responses of L2 ort rescue neurons to the $\mathrm{A}$ and the $\mathrm{B}$ steps were not exactly the same (Figure 33I-J). However, ANOVA comparison revealed that plateau responses to the A steps were not significantly different from each other. The same was true for responses to the B step. When plotting plateau responses as a function of luminance, responses to the A step did not end at the exact same amplitude as the responses to the B step at the close by luminance (Figure 33J). However, responses between the A and the B steps at the close by luminance were not significantly different. This result suggests that L2 plateau responses in L2 ort rescue neurons do carry some luminance information. 


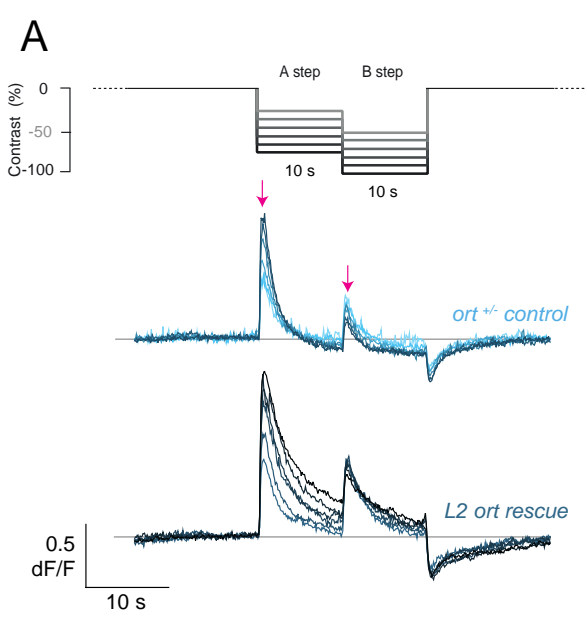

B
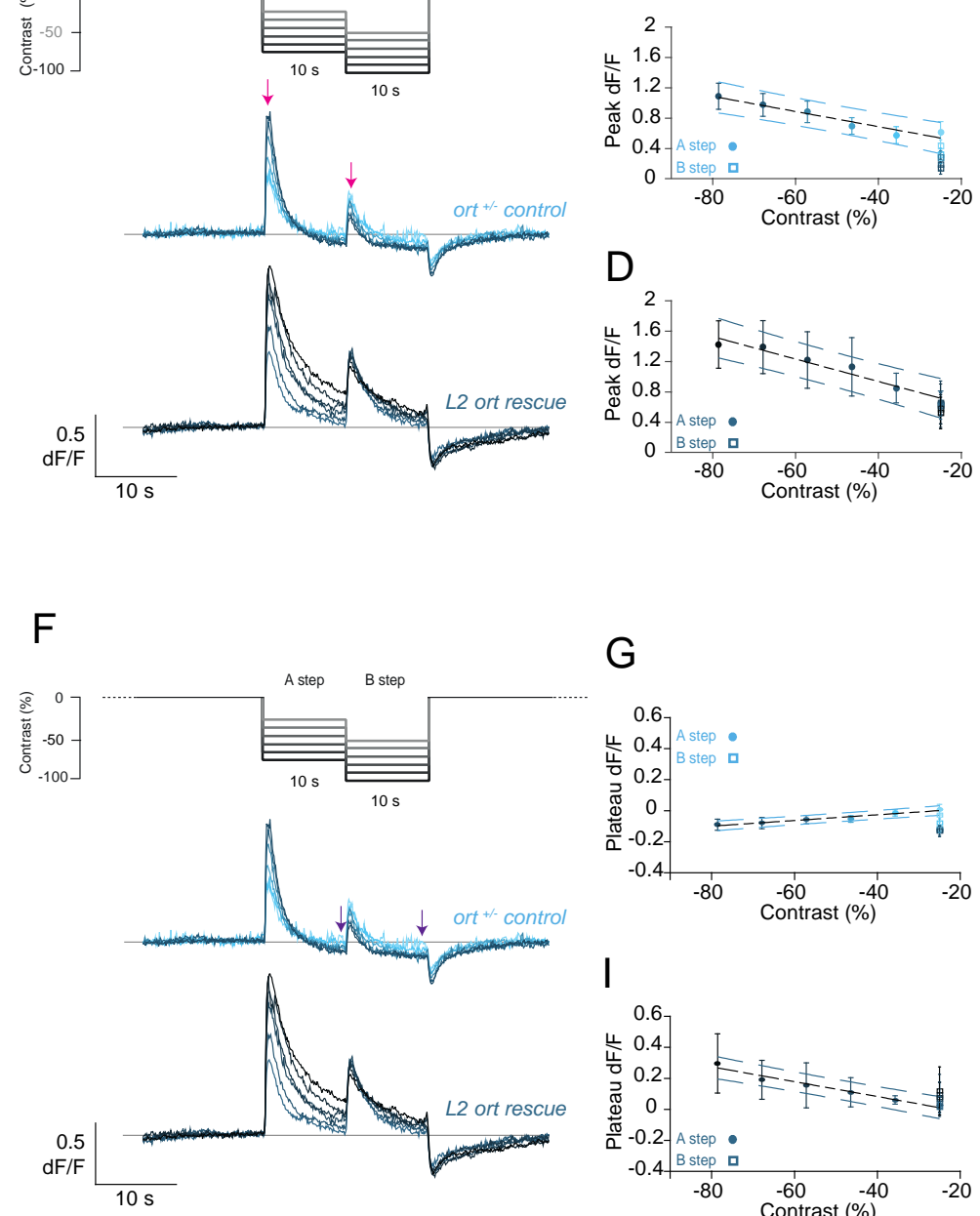

G
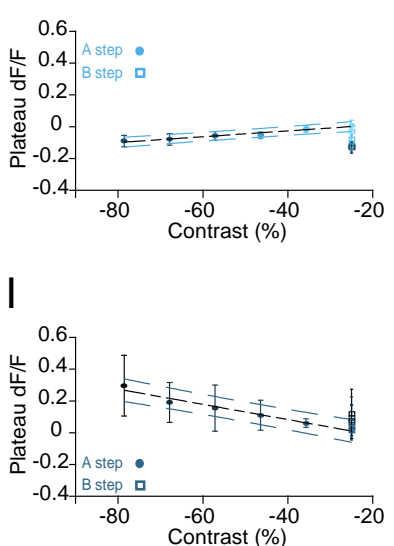

C

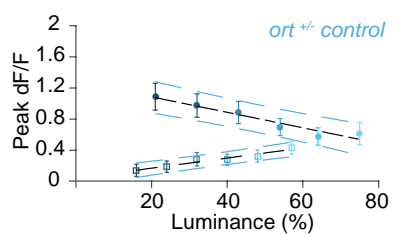

E

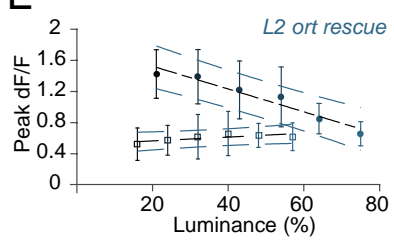

$\mathrm{H}$
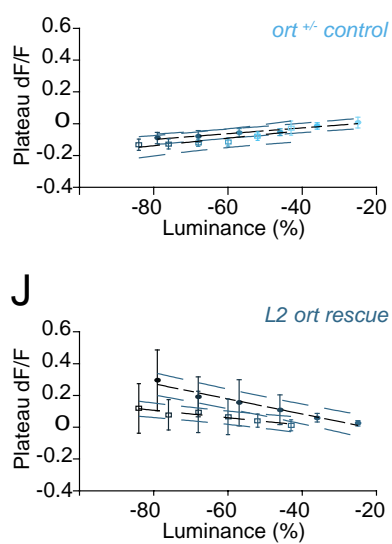

Figure 33. Baseline of genetically isolated L2 neurons is not purely contrast-sensitive.

(A) Schematic of the stimulus, consisting of a $30 \mathrm{~s}$ adapting bright period, followed by two sequential OFF steps, each $10 \mathrm{~s}$ long. The light intensity of the step is illustrated by the gray-scale of the trace. Average calcium signals of L2 heterozygous ort control (above) and L2 ort rescue (bellow). Arrows indicate the peak L2 responses, which were taken for the consequent analysis. (B-E) Peak calcium responses of L2 heterozygous ort control $(B, C)$ and L2 ort rescue $(D, E)$ plotted as a function of contrast (B,D) or luminance (C,E). A linear regression model (black dashed line) was fit to the responses to the A step (B,D) or to the A and B steps individually (C,E). The dashed lines indicate the $95 \%$ confidence interval. Responses to the A step are illustrated by circles, responses to the B step by squares. (F) Schematic of the stimulus, as in (A). Average calcium signals of L2 heterozygous ort control (above) and L2 ort rescue (bellow). Arrows indicate the plateau L2 responses, which were taken for the consequent analysis. (G-J) Plateau calcium responses of L2 heterozygous ort control (G,H) and L2 ort rescue (I,J) plotted as a function of contrast (G,I) or luminance $(\mathrm{H}, \mathrm{J})$. A linear regression model (black dashed line) was fit to the responses to the A step $(\mathrm{G}, \mathrm{I})$ or to the $\mathrm{A}$ and B steps individually $(\mathrm{H}, \mathrm{J})$. The dashed lines indicate the $95 \%$ confidence interval. Responses to the A step are illustrated by circles, responses to the B step by squares. $\mathrm{N}=8(66)$ for L2 ort rescue and $\mathrm{N}=9(80)$ for L2 heterozygous control in (A-J). 
Finally, we noted that when ort was overexpressed in L2, L2 responded to light flashes in a transient manner, even more transient than responses recorded from control L2 neurons (Figure 34).

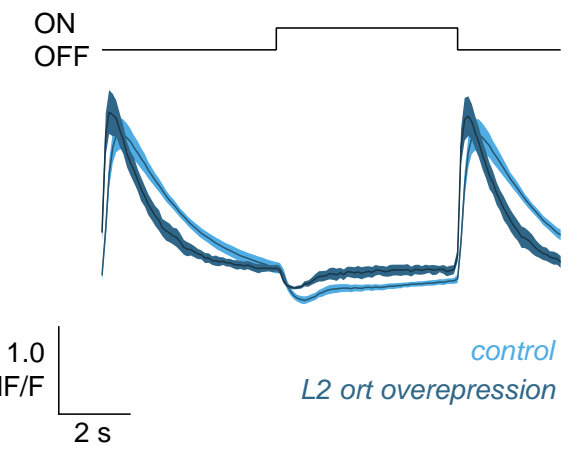

Figure 34. ort overexpression is not responsible for the plateau component in genetically isolated L2 Calcium responses of L2 control (light blue) and ort overexpression in L2 (dark blue) to periodic $5 \mathrm{~s}$ fullfield flashes. $\mathrm{N}=5$ (39) for L2 control flies, $\mathrm{N}=10$ (156) for L2 ort overexpression.

\subsubsection{L3 properties do not depend on circuit interactions.}

We next wanted to understand how L3 neurons obtain their distinct sustained and luminance-sensitive physiological properties. As with L2, we first recorded calcium signals from different cellular regions of L3 neurons in order to identify whether subcellular transformations shape the L3 signal (Figure 35A). We observed only a slight difference in response amplitude between dendrites, axons and axon terminals. In addition, the shape of the response had the same sustained kinetics in all of the three regions (Figure 35A). Thus, L3 showed sustained responses throughout the neuron. Furthermore, amplification of the responses in dim light was already present at the level of dendrites. However, this became more pronounced along the neuron (Figure 35B). This argues that while kinetics of the response does not change along the neuron, a nonlinear decline in signal amplitude with increasing luminance appears to become more pronounced along the neuron. 
A

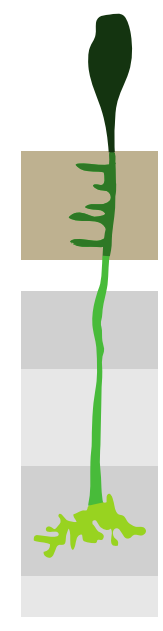

ON
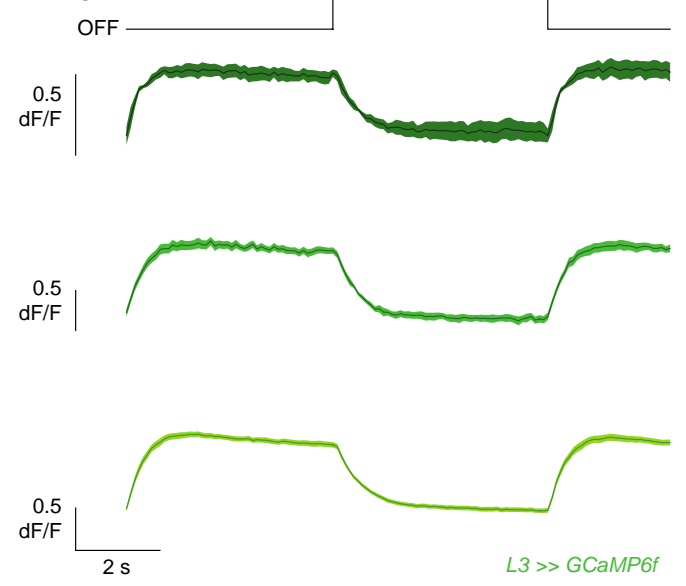

B

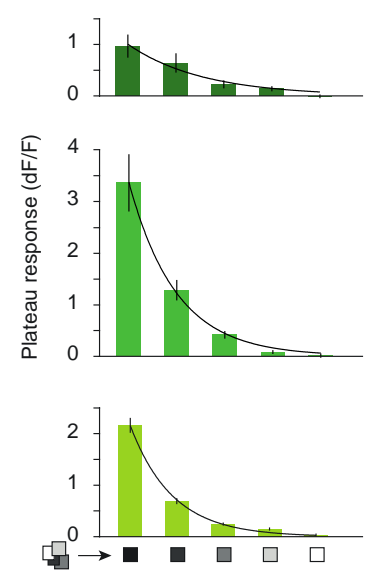

Figure 35. L3 neurons show sustained responses throughout the neuron.

(A) Average calcium signals ( $\mathrm{dF} / \mathrm{F}$ ) recorded from different regions of L3 neurons: dendrites (dark green), axons (green) and axon terminals (light green). $\mathrm{N}=4$ (28) for L3 dendrites, $\mathrm{N}=14$ (66) for L3 axons and $\mathrm{N}=$ 20 (224) for L3 axon terminals. (B) Bar plots showing the plateau calcium response of L3 neurons recorded from different regions: dendrites, axons and axon terminals. $N=4$ (20) for L3 dendrites, $N=11$ (49) for L3 axons and $\mathrm{N}=31$ (512) for L3 axon terminals.

We next tested whether L3 luminance sensitivity is circuit-dependent. To test this, we performed an ort rescue experiment, as described above (Figure 31,32). In an ort null mutant background, L3 neurons showed very little response to light flashes (Figure 36A). Using the Gal4-UAS expression system, we selectively rescued ort expression in L3 and thus isolated it postsynaptically. When we recorded calcium signals from these genetically isolated L3 neurons, we observed a slightly increased response amplitude compared to heterozygous controls, but there were no differences in the shape of the responses (Figure 36A). Furthermore, when we tested the luminance sensitivity of L3 ort rescue neurons using stimulus consisting of flashes of different intensities, L3 ort rescue neurons showed similar responses as heterozygous controls. In both cases, L3 neurons were particularly active in dim light and showed sustained responses, which can already be seen in the responses of single axon terminals (Figure 36B). As wild-type L3 neurons, L3 ort rescue neurons were most active in dim light and showed a nonlinear increase in signal amplitude with decreasing luminance (Figure 36C). Since we did not observe any difference in luminance-sensitivity of L3 ort rescue neurons in comparison to the heterozygous control, we did not show the two consecutive OFF steps stimulus. Taken together, these findings argue that L3 properties, e.g. sustained responses and higher activity in dim light, are established at the photoreceptor-to-L3 synapse. 
A
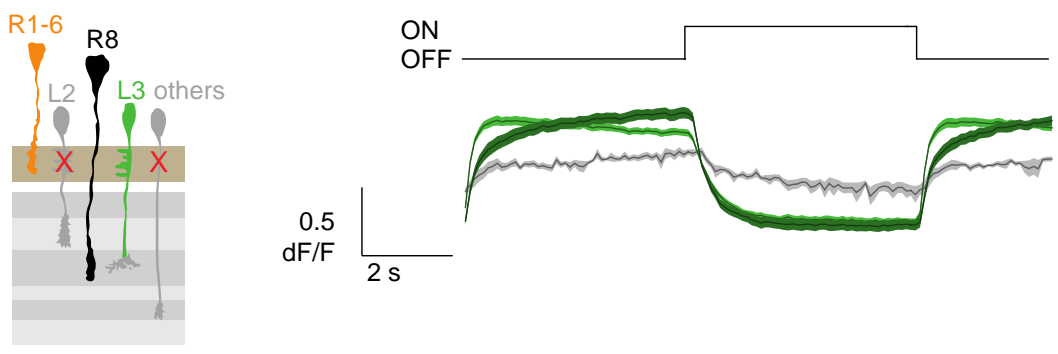

ort - - control

ort + - control

L3 ort rescue

B

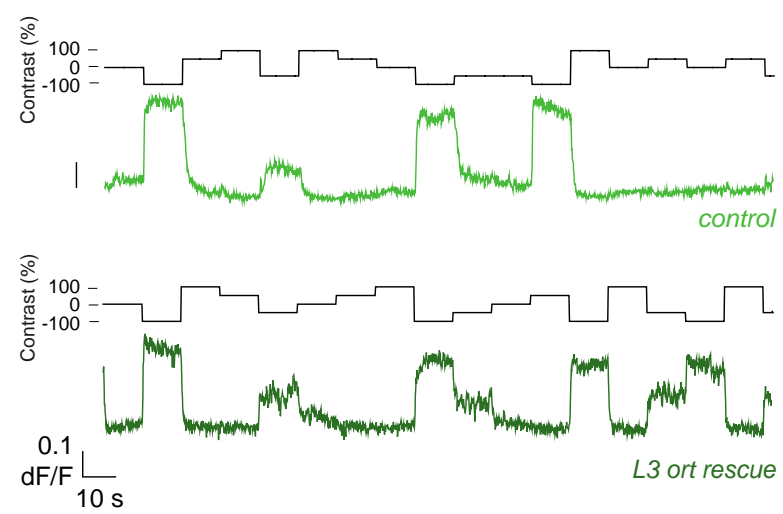

C

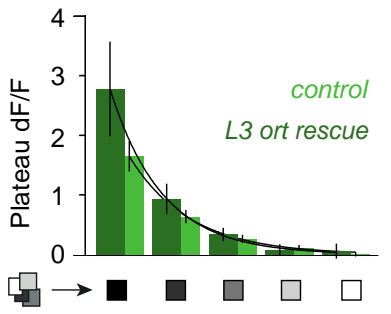

Figure 36. L3 properties do not depend on circuit interactions.

(A-C) L3 ort rescue experiment. ort null ( $\left.\operatorname{ort}^{1} / \operatorname{ort}^{\mathrm{Df}}\right)$ mutants are shown in light gray, heterozygous L3 control responses are shown in light green and L3 ort rescue responses are shown in dark green. In L3 ort rescue, L3 is the only lamina neuron receiving functional input from photoreceptors. (A) Calcium signals recorded from L3 to $5 \mathrm{~s}$ full-field flashes. $\mathrm{N}=18(193)$ for heterozygous ort control, $\mathrm{N}=6(39)$ for ort null mutants, and N = 11(145) for L3 ort rescues. (B) Calcium signals recorded from single L3 axon terminals. L3 responses in an L3 ort rescue resemble control responses. (C) Bar plots showing the plateau calcium response of L3 ort rescues and heterozygous control axon terminals across different luminance, pooled across all steps. $\mathrm{N}=12$ (91) for L3 ort rescue and $\mathrm{N}=10$ (73) for heterozygous controls.

\subsubsection{L3 physiological properties are established at its postsynaptic site and depend on the transcription factor $d F e z f$}

We next wanted to understand the synaptic mechanisms that shape L3 responses. These could be cell-autonomous properties of L3, or differences in the photoreceptor input onto L2 and L3. Given that R1-R6 synapses onto both lamina neuron types within one tetrad synapse, the former possibility seems more likely. We therefore sought to concentrate on the analysis of differentially expressed genes between L2 and L3 neurons. To do so, we followed two distinct approaches: (1) We screened for the functional role of differentially expressed genes between L2 and L3, which could have a role in regulating neuronal properties based on their known molecular function. (2) We tested the role of a candidate gene with known L3-specific functions. For both, approaches, we relied on data from an RNA-seq experiment that was performed in order 
to resolve the transcriptome of developing R7, R8, and L1-L5 (Tan et al., 2015). Transcriptome data showed that every cell type expresses an individual gene pattern (Figure 37A, from Tan et al., 2015). This study concentrated on genes regulating a specific development aspect: layer specific targeting. RNA-seq revealed that Drosophila Fezf ( $\mathrm{dFezf}$ ) is expressed specifically in L3 and is required for cell-type specific targeting (Figure 37B).

A

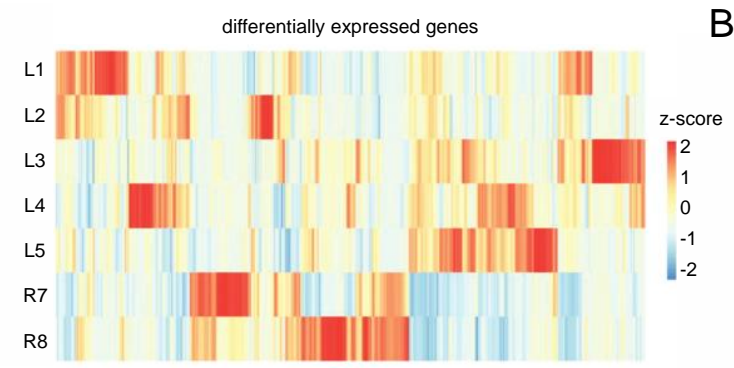

B

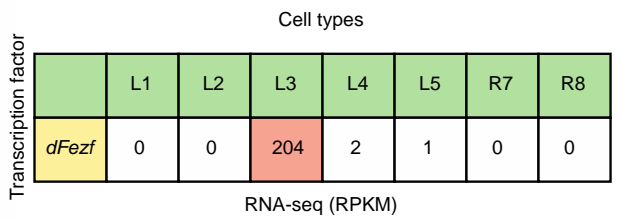

Figure 37. $d F e z f$ is selectively expressed in $\mathrm{L} 3$ neurons.

(A) Heat map showing expression of all genes encoding cell surface membrane and secreted molecules in at least one cell with the reads per kilobase of a specific mRNA per million reads (RPKM) $>5(\mathrm{n}=444)$. (B) RPKM values of $d F e z f$ transcript showing that $d F e z f$ is expressed only in the L3 cell type. Taken from Tan et al., 2015.

Genetic studies in which they disrupted $d F e z f$ function and assessed the morphologies of the neurons revealed the requirement of $d F e z f$ in L3 layer specificity and morphology of L3 axon terminals. Axon terminals of L3 neurons lacking dFezf function terminate in the wrong layers, either layer M1 or M2, and not in normal M3 layer (Peng et al., 2018). Furthermore, when dFezf is mis-expressed in L2 neurons, most of these neurons incorrectly innervate the xM3 layer. Thus, dFezf is involved in directing the growth cone targeting. We hypothesized that dFezf might also controls transcription of L3-specific genes that regulate cellular physiology. Therefore, we first tested whether dFezf is sufficient to generate L3-like properties. To do so, we ectopically expressed $d F e z f$ in L2. When we mis-expressed dFezf in L2, the terminals of L2 changed their shape and became more L3 looking (Figure 38A,B). However, this change in the terminal shape did not change L2 physiological properties. L2>>dFezf overexpressing flies showed an increase in response amplitude, but the response kinetics was similar to controls (Figure 38C). When L2>>dFezf overexpressing flies were shown flashes of different intensities responses were transient and returned to one common baseline, as did the responses of control L2 neurons (Figure 38C,D). When we presented two consecutive OFF steps (see Figure 20A), L2>>dFezf neuron responses to the B step, the $25 \%$ contrast, were not indistinguishable from each other like control L2 neurons. Response amplitude 
was smaller in the darker luminance and higher in the brighter luminance. However, $L 2 \gg>d F e z f$ neurons did not become luminance sensitive (Figure 38F-J). Thus, dFezf is not sufficient to establish L2-specific response properties.

A

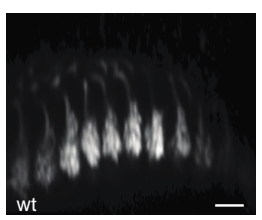

B

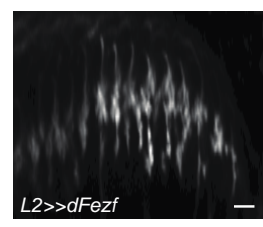

C

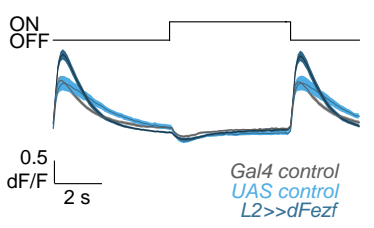

D

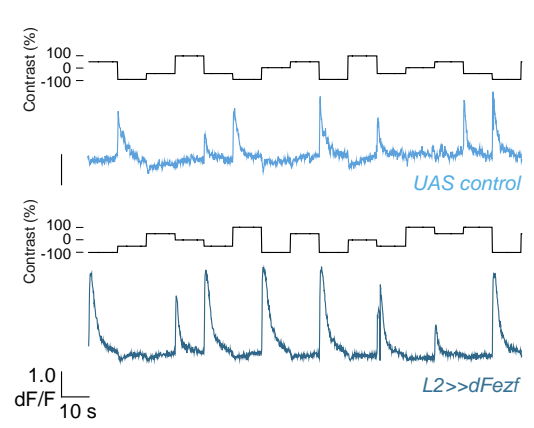

$\mathrm{F}$

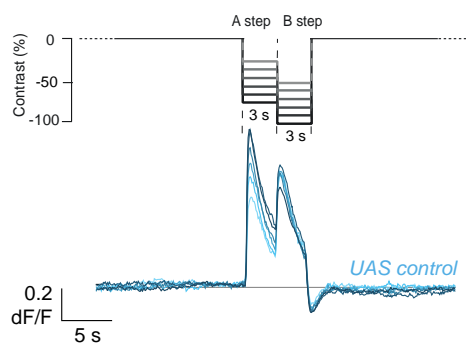

G
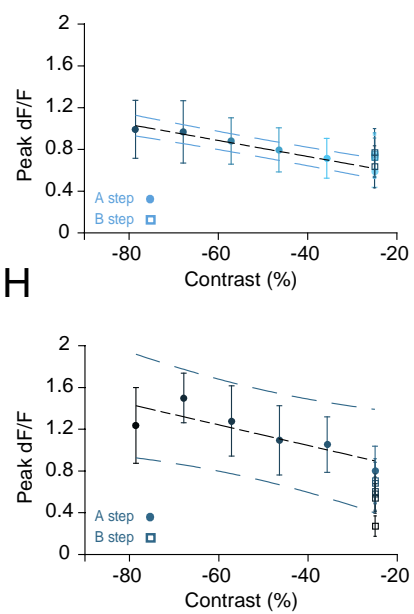

E

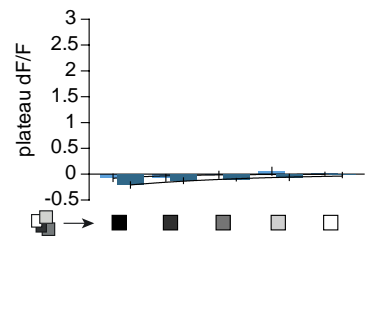

I
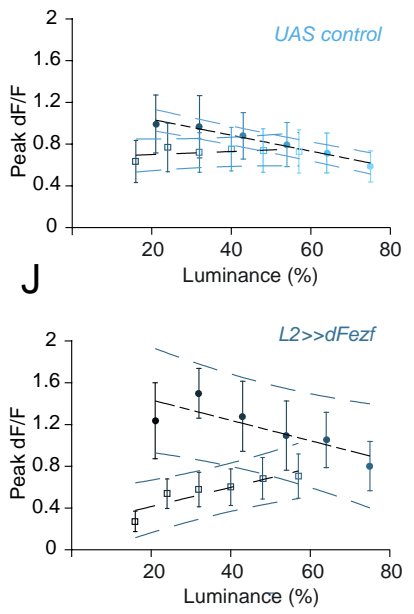

Figure 38. dFezf is not sufficient for luminance sensitivity in $\mathbf{L 2}$.

(A-B) In vivo 2-photon image of wild-type (A) and L2>>dFezf gain of function (B) expressing GCaMP6f. Scale bars are $10 \mu \mathrm{m}$. (C) Average calcium responses of L2 from L2>>dFezf gain of function (dark blue), UAS (light blue) and Gal4 control (gray) to $5 \mathrm{~s}$ full field flashes. $\mathrm{N}=8$ (65) for the UAS control, $\mathrm{N}=14$ (200) for the Gal4 control and $\mathrm{N}=16$ (211) for $L 2>>d F e z f 1$. (D) Calcium signals recorded from single L2 axon terminals from wild type (light blue) and L2 dFezf overexpression (dark blue), in response to flashes of different intensities. (E) Bar plots showing the plateau calcium response of wild type (light blue) and L2>>dFezf gain of function (dark blue) across different luminance, pooled across all steps. $\mathrm{N}=4$ (60) for the UAS control and $\mathrm{N}=3$ (40) for L2>>dFezf. (F) Schematic of the stimulus, consisting of an adapting bright period, followed by two sequential OFF steps. Below, average calcium signals of wild type (light blue) and at the bottom, average responses of $L 2>>d F e z f$ (dark blue) are shown in response to the stimulus shown above. (G-J) Calcium signals $(\mathrm{dF} / \mathrm{F})$ in response to the stimulus shown in $(\mathrm{F})$. Responses to the A step are illustrated by circles, 
responses to the B step by squares. Peak calcium signals of wild type (G,I) and $L 2>>d F e z f(H, J)$ are plotted as a function of contrast $(\mathrm{G}, \mathrm{H})$ or luminance $(\mathrm{I}, \mathrm{J})$. A linear regression model (black dashed line) was fit to the responses to the A step in $(\mathrm{G}, \mathrm{H})$ or to the $A$ and $B$ steps individually $(\mathrm{I}, \mathrm{J})$. The light blue and dark blue dashed lines indicate a 95\% confidence interval. $\mathrm{N}=7$ (72) for the UAS control and $\mathrm{N}=6$ (64) for L2>>dFezf in (F-J).

To test if $d F e z f$ is required for L3 physiological properties, we generated homozygous mutant $d$ Fez $f$ clones specifically in L3 in an otherwise heterozygous background using the MARCM technique (see Introduction 2.6.4.1.2) (Lee and Luo, 1999). Using this technique, we generated single, wild type or $d F e z f$ mutant L3 cells in an otherwise heterozygous background, which were furthermore labeled using GCaMP6f (see Materials and Methods). These control or mutant clones were dominantly labeled with GCaMP expression (Figure 39A,B), allowing us to record visual response properties. The $d F e z f^{1}$ null allele comprises a sole $\mathrm{A}$ to $\mathrm{T}$ nucleotide change. Therefore leucine is substituted by a histidine (Weng et al., 2010). We first presented flies with full-field flashes and recorded from control L3 and L3 dFezf mutant neurons. L3 dFezf mutant possessed slightly more transient responses than control L3 neurons (Figure 39C). Only when these flies were presented with flashes of different intensities, we observed much more transient responses of $d F e z f$ mutant L3 cells than control L3 neurons (Figure 39D). Strikingly, the sustained response was largely missing (Figure 39E). This also shows that one should use more sophisticated stimuli such as "contrast steps from adapted background" or "flashes of different intensity" in order to test the role of candidate genes in shaping L2 or L3 physiological responses. We then explicitly tested luminance and contrast sensitivity by presenting the two different consecutive OFF step protocol (see Figure 20A, Figure 39F-J). Control L3 neurons responded to the 25\% step differently (Figure 39G). Plotting responses as a function of luminance revealed the luminance-sensitivity of L3 neurons in control flies, consistent with what has been shown before (Figure 20, Figure 39I). Instead, responses of L3 dFezf mutant neurons to the $25 \%$ contrast steps were indistinguishable from each other and luminance was no longer the unifying feature between responses to the A and B steps (Figure 39H,J). These responses strongly resembled wild-type L2 responses (Figure 20B-D, Figure 39F). This demonstrates that $d F e z f$ mutant L3s are contrast sensitive. Together, our data argue that L3 specific properties are established on the postsynaptic site in L3, and that cellautonomous mechanisms involving transcriptional targets of dFezf mediate L3 neuronal response properties. 


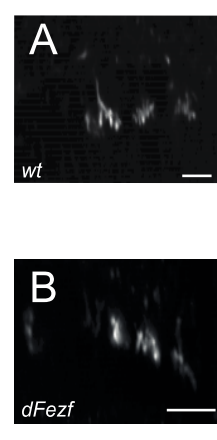

$\mathrm{F}$

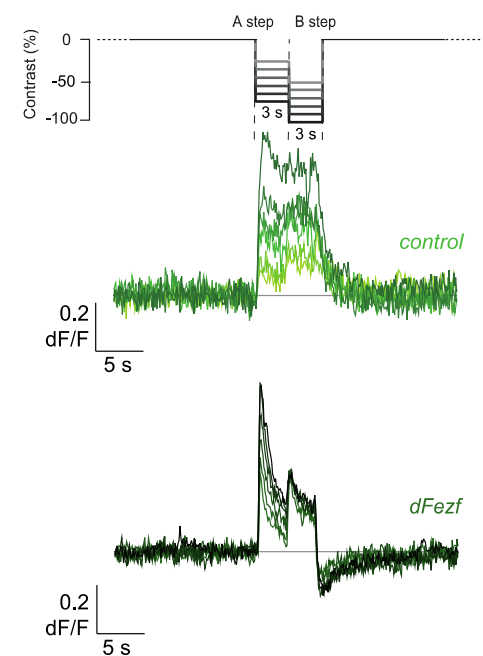

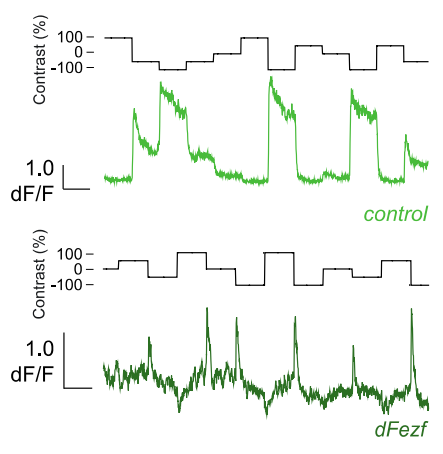

E

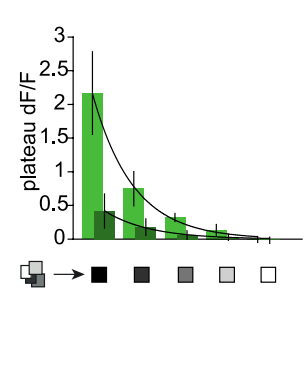

G

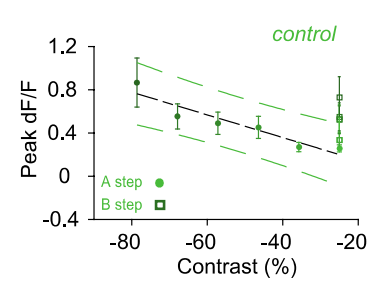

I
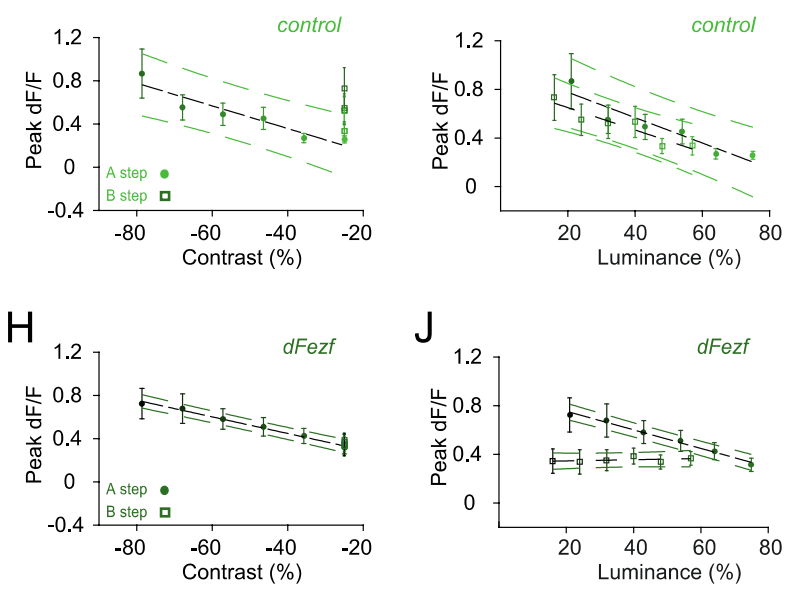

Figure 39. Luminance sensitivity in L3 depends on the dFezf transcription factor.

(A-B) In vivo 2 photon image of wild-type (A) or $d F e z$-mutant (B) L3 MARCM clones, dominantly marked by the expression of GCaMP6f. Scale bars are $10 \mu \mathrm{m}$. (C) Calcium signals recorded from single L3 axon terminals from wild-type (light green) and $d F e z f-\mathrm{L} 3$ mutant (dark green) MARCM clones, in response to fullfield flashes. $\mathrm{N}=9$ (21) for wild-type and $\mathrm{N}=12$ (30) for dFezf-mutants. (D) Calcium signals recorded from single L3 axon terminals from wild-type (light green) and dFezf-L3 mutant (dark green) MARCM clones, in response to flashes of random intensity. (E) Bar plots showing the plateau calcium response of wild- type and dFezf L3 MARCM clones (dark green) across different luminance, pooled across all steps. $\mathrm{N}=8$ (21) for wild-type and $\mathrm{N}=12$ (43) for $d F e z f$ mutant L3 neurons. (F) Schematic of the stimulus, consisting of an adapting bright period, followed by two sequential OFF steps. Average calcium signals of wild-type (light green) and of $d F e z f$ L3 MARCM clones (dark green) are shown. Darker traces correspond to OFF steps of larger amplitude. (G-J) Peak calcium signals of wild-type (F,H) and $d F e z f(G, I)$ L3 MARCM clones are plotted as a function of contrast $(\mathrm{F}, \mathrm{G})$ or luminance $(\mathrm{H}, \mathrm{I})$. Responses to the A step are illustrated by circles, responses to the B step by squares. A linear regression model (black dashed line) was fit to the responses to the A step in $(F, G)$ or to the A and B steps individually $(\mathrm{H}, \mathrm{I})$. The green dashed lines indicate the $95 \%$ confidence interval. $\mathrm{N}=9$ (24) for wild-type and $\mathrm{N}=12$ (47) for $d$ Fezf-mutants in (E-I).

What are the mechanisms underlying transient calcium responses in L3 dFezf mutant neurons? Physiological properties of L3 appear to be distinct from its role in layerspecific targeting, because even though overexpression of $d F e z f$ in L2 mediates 
mistargeting to the M3 layer, L2>>dFezf neuron responses did not become luminance sensitive. This thus suggests that $d F e z f$ has separate roles in determining L3-specific physiological properties and axon targeting. To explicitly test whether physiological properties of L3 neurons are a result of mistargeting, we performed an experiment using an adult specific $d F e z f$ loss of function in L3 neurons, using $d F e z f$ RNAi. To do so, we used a different L3 driver line, which is adult specific. Therefore, RNAi will prevent expression of dFezf in L3 only in the adulthood, resulting in normal, L3 layer-specific targeting. Responses of $L 3>>d F e z f^{R N A i}$ neurons showed similar responses to control L3 neurons to all stimuli tested (Figure 40A-H). L3>>dFezfRNAi showed sustained responses to sustained input (Figure 40A). As control L3 neurons (Figure 15F,G), their responses increased to the gray-to-OFF step, but basically did not respond to the offset of light when returning to background grey following an ON step (Figure 40B,C). In response to flashes of different intensities, $L 3>>d F e z f^{R N A i}$ neurons showed normal luminance sensitivity in which sustained response to intensity steps varied with luminance and were most active at the lowest luminance (Figure 40D,E). Furthermore, when we presented two different consecutive OFF steps (Figure 40F-H), L3>>dFezfRNAi responses to the various $25 \%$ step stimuli differed (Figure $40 \mathrm{G}$ ), whereas calcium signals were similar to nearby luminances (Figure $40 \mathrm{H}$ ), indicating that calcium responses in L3 are sensitive to luminance but not contrast.

This result is clearly different than when we imaged from dFezf mutant L3 clones (Figure 39). There are several explanations for this discrepancy. First, $d F e z f$ RNAi might either be weak and could produce strong hypomorphic situation. However, previous research showed that the dFezf RNAi expression in the lamina eradicated L3 dFezf immunoreactivity (Peng et al., 2018). But, the flies used in the above-mentioned study expressed Dicer-2 (Dcr-2). Dcr-2 belongs to a family of multidomain RNase III enzymes and generates siRNA (Bernstein et al., 2001; Liu et al., 2003). Dcr-2 is often used in RNAi screens to strengthen the RNAi effect, but on the other hand it also phenotypes, which are not specific and arise due to the off-target effect, which is also one of the major limitations of the RNAi approach in general (Jonchere and Bennett, 2013; Kulkarni et al., 2006; Ma et al., 2006). The lack of $d r c-2$ might weaken the knockdown, and the experiment should be repeated with the presence of $d r c-2$. Third, the RNAi works well in adults, but the important $d F e z f$ gene targets are already expressed during pupal stages and have long enough protein perdurance. In other words, if the preexisting protein has a long half-life, decreasing the gene expression at the RNA level may lead to minimal consequences for a period of time (Ying, 2013). 
To conclude, we did not achieve to unambiguously separate the axon targeting from physiological phenotypes. However, a few data sources still argue that dFezf plays a specific role in regulating functional properties (see discussion) and further experiments will be necessary in order to test this.

A

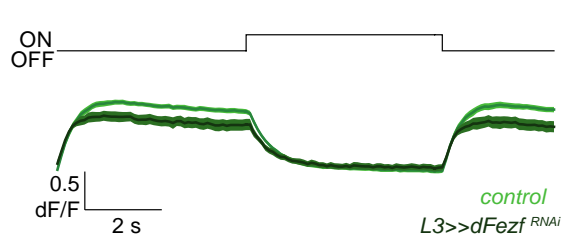

D

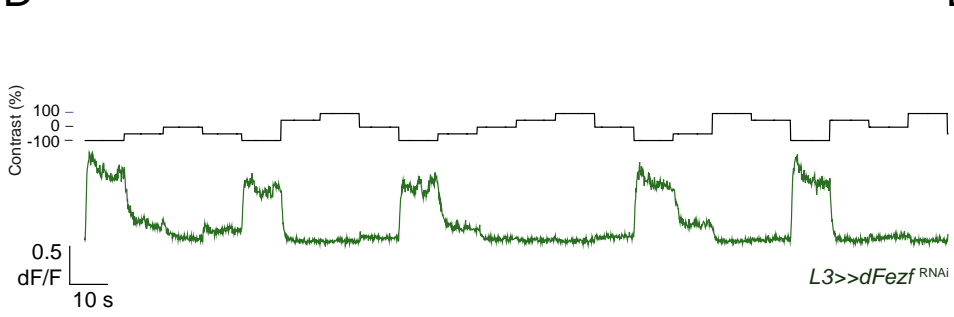

$E$
$\mathrm{F}$

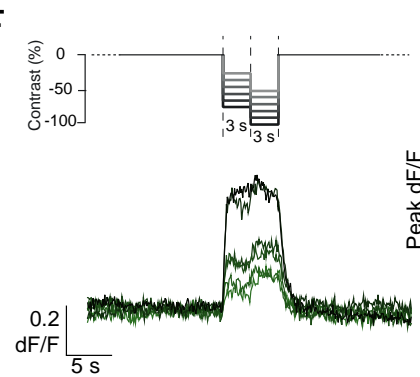

B

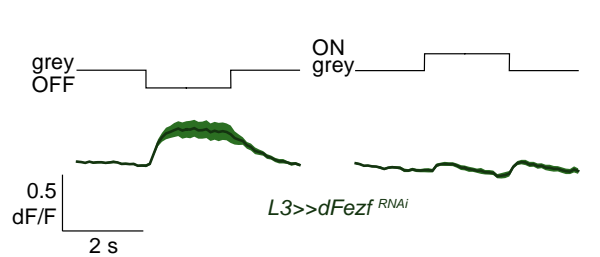

C

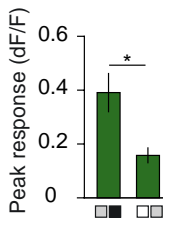

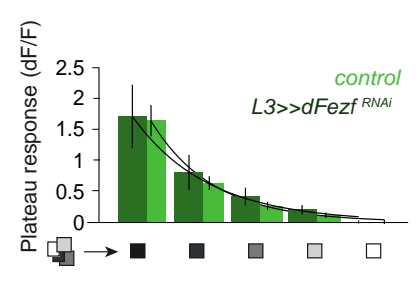

G

H
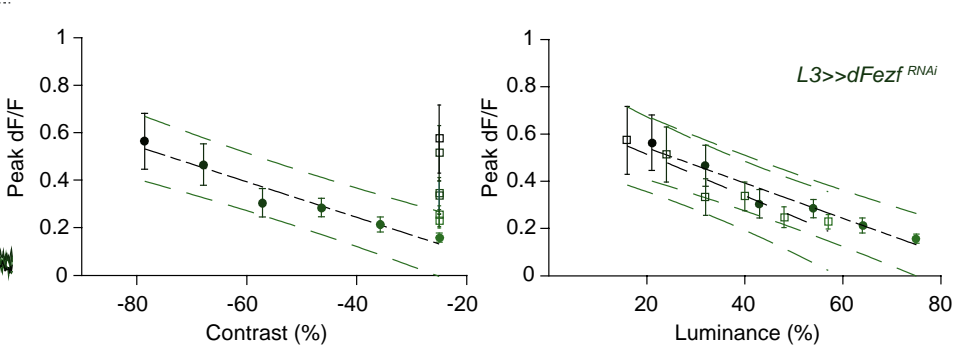

Figure 40. L3 dFezf ${ }^{\text {RNAi }}$ neurons are not contrast-sensitive.

(A) Average calcium responses of L3 measured from L3 control (light green) and L3>>dFezfRNAi (dark green) neurons to $5 \mathrm{~s}$ full field flashes. $\mathrm{N}=20$ (224) for the L3 control and $\mathrm{N}=10$ (49) for the L3 dFezfRNAi. (B) Calcium signals measured from L3 control and L3 dFezfRNAi neurons in response to ON and OFF flashes from a gray background. (C) Bar plots showing the quantification of the peak responses of $L 3>>d F e z f^{R N A i}$ to the two OFF steps shown in (C): ON-to-gray and gray-to-OFF. ${ }^{*} \mathrm{p}<0.05$, tested with two-tailed Student $t$ tests. $\mathrm{N}=$ 10 (76) for $L 3>>d F e Z f^{R N A i}$ in (B-C). (D) Calcium signals recorded from $L 3>>d F e z f^{R N A i}$ neurons in response to flashes of random intensity. (E) Bar plots showing the plateau calcium response of control L3 axon terminals and $L 3>>d F e z f^{R N A i}$ across different luminance, pooled across all steps. $\mathrm{N}=8$ (21) for control L3 axon terminals and $N=6$ (43) for $L 3>>d F e z f^{R N A i}$ neurons. (F) Schematic of the stimulus, consisting of an adapting bright period, followed by two sequential OFF steps. Average calcium signals of $L 3>>d F e z f^{R N A i}$ neurons are shown. Darker traces correspond to OFF steps of larger amplitude. (G-H) Peak calcium signals of $L 3 \gg>d F e z f^{R N A i}$ neurons are plotted as a function of contrast $(\mathrm{G})$ or luminance $(\mathrm{H})$. Responses to the A step are illustrated by circles, responses to the B step by squares. A linear regression model (black dashed line) was fit to the responses to the A step in $(G)$ or to the A and B steps individually $(H)$. The green dashed lines indicate the $95 \%$ confidence interval. $\mathrm{N}=9$ (55) for $L 3>>d F e z f R N A i$ neurons in $(\mathrm{F}-\mathrm{H})$. 
Another way to test whether L3 responses properties depend on interactions within their target layer is to silence the L3 output. This is not leading to any anatomical phenotypes, but blocks communication of L3 to any other neurons in the M3 layer (and elsewhere). To do so, we expressed the temperature-sensitive allele shibirets ${ }^{\text {tshits }}$, Kitamoto, 2001) and tested L3 responses to full-filed flashes (Figure 41). L3 neurons responded with similar amplitude and response kinetics than control L3 neurons. This again suggests that L3 properties are established cell-autonomously and are not a result of a layer specific axon targeting.

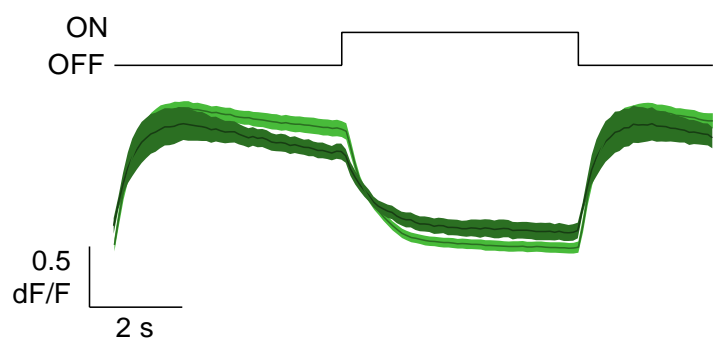

Figure 41. L3 output silenced neurons show sustained responses to flashes.

Average calcium responses of L3 measured from L3 control (light green) and L3>>shits (dark green) neurons to $5 \mathrm{~s}$ full field flashes. $\mathrm{N}=11$ (182) for the L3 control and $\mathrm{N}=9$ (90) for the L3>>shits.

\subsubsection{Preliminary study to identify molecular mechanisms that shape L2 and L3 physiology}

The aim of this study was to perform a screen to uncover mechanisms that shape L2 and L3 physiology. This screen, using RNAi, was initially designed as a preliminary study to identify candidate genes that distinguish these two neurons. In a parallel candidatebased approach, we identified dFezf as an important regulator of L3 function (see Results 4.2.6). However, the downstream gene targets of dFezf that shape L3's distinct postsynaptic properties are still unknown.

\subsubsection{Differentially expressed genes in L2 and L3 neurons}

Recently, a cell-type specific RNA sequencing (RNA-seq) of R7, R8 and L1-L5 was performed in order to identify genes, which regulate synaptic specificity in the medulla (Figure 37A,B). This work identified that each cell type expresses an individual gene pattern and they in their subsequent analysis concentrated on cell surface membrane proteins for further analysis (Tan et al., 2015). We took the original RNA-seq dataset and with a help of bioinformatician (Jesse Lipp, imb Vienna/ Boehringer Inglheim) we mapped reads, identified all genes differentially expressed in L2 and L3 (Figure 42A-B), 
and then narrowed down the list down by selecting candidates that might regulate neuronal physiology.

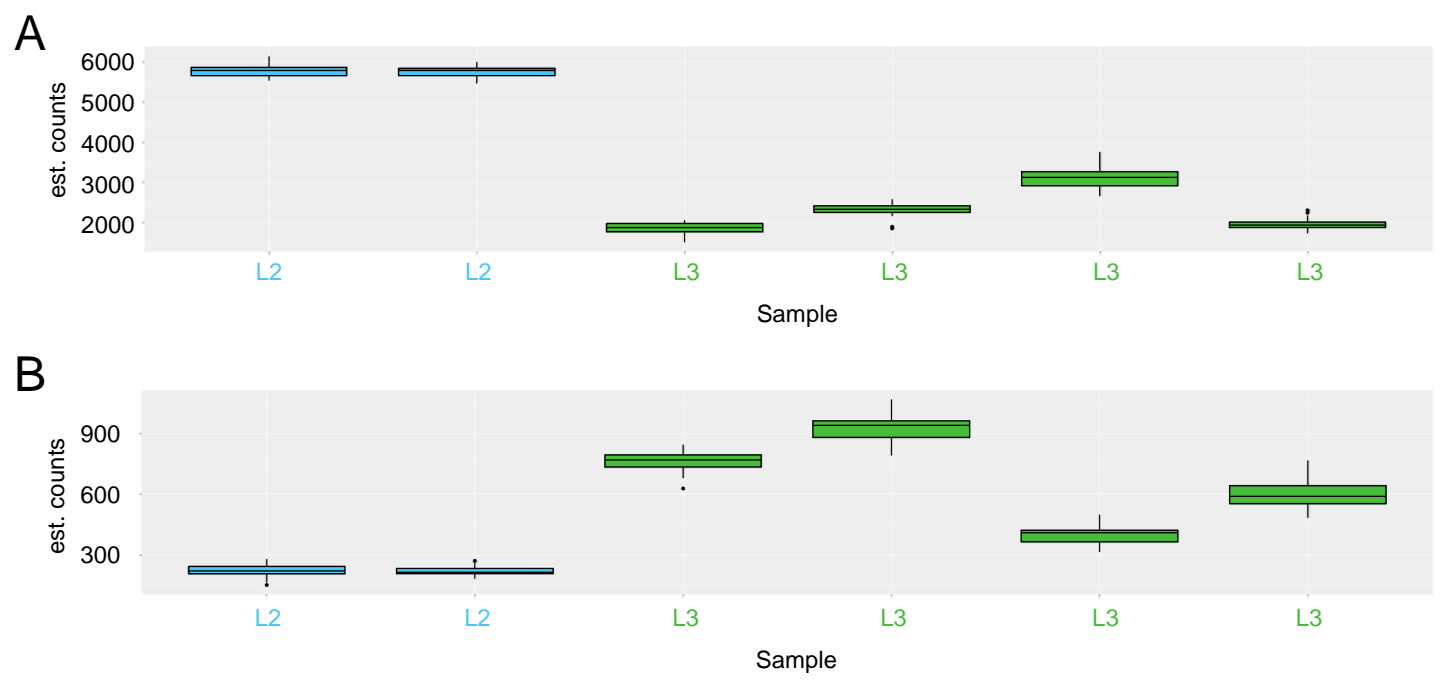

Figure 42. Two of the candidate genes differentially expressed between L2 and L3.

(A) Box plot showing expression of Ih. Ih showed stronger expression in L2 as compared to L3. (B) Box plot showing expression of Ace, which showed stronger expression in L3 as compared to L2. 6 samples were analyzed (two L2 and four L3). Program kallisto and tool sleuth were used to quantify abundances of transcript from RNA-Seq data. Data are from (Tan et al., 2015).

To do so, we used a GO (gene ontology) annotation, which included key words like receptors, channels, calcium etc. This way, we ended with a list of 61 candidates, differentially expressed between L2 and L3 neurons. It is worth mentioning that the RNA-seq was performed on pupal brain tissue at 40 hours after pupal formation (Tan et al., 2015) and not in adult flies, thus some genes might be missing. However, many genes known to regulate visual system function were already expressed at this developmental age (see e.g. Table 3). We then checked whether there are RNAi fly lines available for silencing gene expression and thus we ended up with a final list of 33 candidates that we could functionally test (Table 3). Out of these candidate genes, 26 genes were predominantly expressed in L3, whereas 7 genes had stronger expression in L2 than L3. To assess the possible functional role of our candidates, we used RNAi knockdown of candidate genes specifically in L2 or L3 and recorded calcium signals from axon terminals of either L2 or L3 neurons. In other words, if a candidate gene was expressed in L3, we disrupted the gene in L3 and imaged from these L3 neurons.

Table 3. Shortlist of candidate genes differentially expressed in L2 and L3 neurons.

'Symbol' is the name of the candidate gene. 'Expressed in' tells in which neuron, L2 or L3, the candidate gene is expressed more strongly. 'p-value' shows how significant is the differential expression. In the 'Flybase 
number' column, the flybase ID of the candidate gene is written. 'Name' is the full name of the candidate gene. 'Function' is a very short description of the function of the candidate gene.

\begin{tabular}{|c|c|c|c|c|c|}
\hline Symbol & Expressed in & p-value & Flybase number & Name & Function \\
\hline Ace & L3 & $1,52 \mathrm{E}-37$ & FBgn0000024 & Acetylcholine esterase & acetylcholine receptor \\
\hline Best1 & L3 & $9,75 \mathrm{E}-05$ & FBgn0040238 & Bestrophin 1 & $\begin{array}{l}\text { calcium-activated anion } \\
\text { channels }\end{array}$ \\
\hline btsz & L3 & $9,76 \mathrm{E}+04$ & FBgn0266756 & bitesize & synaptotagmin-like protein \\
\hline eag & L3 & $1,05 \mathrm{E}-16$ & FBgn0000535 & ether a go-go & potassium \\
\hline Galphai & L3 & $6,17 \mathrm{E}-01$ & FBgn0001104 & G protein $\alpha$ i subunit & G protein $\alpha$ i subunit \\
\hline GluRIA & L3 & $2,58 \mathrm{E}+04$ & FBgn0004619 & Glutamate receptor IA & glutamate \\
\hline Grd & L3 & $4,39 \mathrm{E}+04$ & FBgn0001134 & Glycine receptor & GABA \\
\hline HisCl1 & L3 & $1,70 \mathrm{E}+04$ & FBgn0037950 & $\begin{array}{l}\text { Histamine-gated chloride } \\
\text { channel subunit } 1\end{array}$ & histamine \\
\hline $\mathrm{Hk}$ & L2 & $4,74 \mathrm{E}-29$ & FBgn0263220 & Hyperkinetic & potassium \\
\hline Ih & L2 & $4,74 \mathrm{E}-29$ & FBgn0263397 & Ih channel & potassium \\
\hline Ir76a & L2 & $1,71 \mathrm{E}+03$ & FBgn0260874 & Ionotropic receptor $76 a$ & $\begin{array}{l}\text { Ionotropic glutamate } \\
\text { receptor }\end{array}$ \\
\hline mAChR-A & L3 & $2,68 \mathrm{E}-42$ & FBgn0000037 & $\begin{array}{c}\text { muscarinic Acetylcholine } \\
\text { Receptor, A-type }\end{array}$ & acetylcholine receptor \\
\hline $\mathrm{mtt}$ & L3 & $7,27 \mathrm{E}-43$ & FBgn0050361 & mangetout & $\begin{array}{c}\text { metabotropic glutamate } \\
\text { receptor }\end{array}$ \\
\hline nAChRalpha1 & L3 & $6,95 \mathrm{E}-03$ & FBgn0000036 & $\begin{array}{c}\text { nicotinic Acetylcholine } \\
\text { Receptor } \alpha 1\end{array}$ & acetylcholine receptor \\
\hline nAChRalpha2 & L3 & $1,61 \mathrm{E}-02$ & FBgn0000039 & $\begin{array}{c}\text { nicotinic Acetylcholine } \\
\text { Receptor } \alpha 2\end{array}$ & acetylcholine receptor \\
\hline nAChRalpha3 & L3 & $1,25 \mathrm{E}-16$ & FBgn0015519 & $\begin{array}{c}\text { nicotinic Acetylcholine } \\
\text { Receptor } \alpha 3\end{array}$ & acetylcholine receptor \\
\hline nAChRalpha7 & L3 & $1,90 \mathrm{E}-27$ & FBgn0086778 & $\begin{array}{c}\text { nicotinic Acetylcholine } \\
\text { Receptor } \alpha 7 \\
\end{array}$ & acetylcholine receptor \\
\hline Ndae1 & L3 & $2,55 \mathrm{E}-08$ & FBgn0259111 & $\begin{array}{l}\text { Na+-driven anion } \\
\text { exchanger } 1\end{array}$ & $\begin{array}{c}\text { Na+-driven anion } \\
\text { exchanger }\end{array}$ \\
\hline Oatp30B & L2 & $1,75 \mathrm{E}+03$ & FBgn0032123 & $\begin{array}{c}\text { Organic anion transporting } \\
\text { polypeptide } 30 \mathrm{~B}\end{array}$ & $\begin{array}{c}\text { Organic anion transporting } \\
\text { polypeptide }\end{array}$ \\
\hline Orct & L3 & $2,77 \mathrm{E}-07$ & FBgn0019952 & Organic cation transporter & organic cation transporter \\
\hline Orct2 & L3 & $1,31 \mathrm{E}+04$ & FBgn0086365 & Organic cation transporter 2 & Organic cation transporter \\
\hline $\mathrm{pHCl}$ & L3 & $7,22 \mathrm{E}-54$ & FBgn0003011 & $\mathrm{pHCl}$ & chloride channel \\
\hline Rdl & L3 & $3,75 \mathrm{E}+00$ & FBgn0264908 & Resistant to dieldrin & GABA \\
\hline Shawl & L3 & $3,96 \mathrm{E}-01$ & FBgn0004244 & Shaw-like & potassium \\
\hline Slob & L2 & $1,92 \mathrm{E}-14$ & FBgn0085395 & Slowpoke binding protein & $\begin{array}{l}\text { regulator of calcium-activated } \\
\text { channel Slo }\end{array}$ \\
\hline CG13575 & L3 & $8,35 \mathrm{E}-01$ & FBgn0264087 & & GPCR, rhodopsin-like \\
\hline CG13995 & L3 & $8,24 \mathrm{E}+04$ & FBgn0034996 & & GPCR, rhodopsin-like \\
\hline CG30345 & L3 & $1,14 \mathrm{E}-01$ & FBgn0031770 & & Sugar transporter \\
\hline CG31636 & L3 & $3,80 \mathrm{E}-14$ & FBgn0050345 & & $\begin{array}{c}\text { cellular retinaldehyde-binding } \\
\text { protein }\end{array}$ \\
\hline CG42594 & $\mathrm{L} 2$ & $5,40 \mathrm{E}-08$ & FBgn0051636 & & potassium \\
\hline CG42732 & L3 & $1,91 \mathrm{E}-11$ & FBgn0260971 & & Potassium channel domain \\
\hline CG6836 & L3 & $5,49 \mathrm{E}+01$ & FBgn0261698 & & transporter \\
\hline CG7627 & L2 & $2,59 \mathrm{E}+04$ & FBgn0036834 & & $\mathrm{ABC}$ transporter \\
\hline
\end{tabular}




\subsubsection{Testing candidate genes, which are differentially expressed in L2 and L3 neurons}

We performed in vivo two-photon calcium imaging from L2 or L3 axon terminals in order to assess the possible affect of candidate gene disruption. We did this by expressing RNAi together with GCaMP6f under a Gal4 control. Only 6 candidate genes out of 33 differentially expressed genes have been tested so far. Other candidate genes still have to be tested. As controls we used L2>>GCaMP6f or L3>>GCaMP6f. We first presented the flies with periodic full field flashes and recorded from axon terminals of either L2 or L3 neurons.

In invertebrates, the main neurotransmitter of the photoreceptor cells is histamine (Hardie and Raghu, 2001; Montell, 2012). In Drosophila, two genes have been identified that encode for histamine-gated chloride channels: ora transientless (ort) and histaminegated chloride channel subunit 1 (HisCl1) (Gengs et al., 2002; Witte et al., 2002; Zheng et al., 2006). ort expression was observed in lamina, medulla as well as in lobula neurons, while HisCl1 can be found in glial cells in lamina (Gao et al., 2008; Hong et al., 2006; Pantazis et al., 2008). However, recent work reported HisCl1 expression in R7 and R8 as well (Schnaitmann et al., 2018; Tan et al., 2015). R7 and R8 directly mutually inhibit each other via HisCl1 and in addition receive feedback inhibition from Ort (Schnaitmann et al., 2018). Given the above mentioned functional role of the HisCl1 channel, which is differentially more expressed in L3 than in L2, we decided to test this candidate by silencing gene expression using HisCl1 RNAi. Flies expressing HisCl1 RNAi under the control of L3-Gal4 showed similar responses as controls in both amplitude and response kinetics. Calcium signals in both controls and L3>>HisCl1 ${ }^{\text {RNAi }}$ responded with a calcium signal increase to the OFF and with a decrease to the ON and they showed sustained responses to light flashes over the course of stimulus presentation (Figure 43A). Since full field flashes from an intermediate gray background can show whether L2 or L3 neurons are contrast sensitive (Figure 16F,G), we decided to use this stimulus to initially test the contrast-sensitivity of L3 neurons expressing HisCl1 RNAi. Thus, we presented the flies with a stimulus consisting of intermediate grey flash lasting for $4 \mathrm{~s}$ followed by 2 s long ON or OFF steps. Recordings from the axon terminals showed that L3>>HisCl1 ${ }^{R N A i}$ neurons responded with the same response to the ON-to-gray step and to the gray-to-OFF step than control L3 neurons (Figure 43B-C). This indicates that HisCl1 is not required for sustained and luminance-sensitive L3 responses. 
The second candidate gene tested was ace. ace encodes for acetylcholinesterase (AChE), which is a main enzyme of the cholinergic system. AChE regulates the level of acetylcholine and is also required for normal nervous system (Bender et al., 1983; Greenspan et al., 1980). The inhibition of AChE causes death, which led to development of irreversible inhibitors as insecticides (Aldridge, 1950). AChE is initially expressed in the photoreceptors and then it accumulates in the optic lobes (Wolfgang and Forte, 1989). It has been shown that L3 is cholinergic (Brotz et al., 2001), thus we decided to test if the ace gene is required for sustained L3 responses. We first presented the flies with full-field flashes and recorded calcium signals from $L 3>>a c e^{R N A i}$ axon terminals (Figure 43D). When ace was silenced, L3 neurons responded with sustained kinetics and similar amplitude as controls. The same was true when the flies were presented with ON and OFF flashes from intermediate gray (Figure 43E-F). Thus, ace is most likely not required for sustained and luminance-sensitive L3 responses.

Potassium channels are important regulators of neuronal activity. Research in Drosophila has led to the discovery of four voltage-activated $\mathrm{K}^{+}$channel families: Shaker, Shab, Shaw and Shal (Schwarz et al., 1988). In addition, several other genes encoding $\mathrm{K}^{+}$ channels and their subunits were discovered: ether-a-go-go (eag) (Kaplan and Trout, 1969), eag like $K^{+}$channel (elk) (Warmke and B, 1994), slowpoke (slo) (Elkins et al., 1986). Different channel types have evolved to perform different tasks. One of the K+ channels that was differentially more expressed in L3 than in L2 is Shawl. Shawl belongs to the Shaw family, which encodes a voltage-insensitive, slowly activating, noninactivating $\mathrm{K}^{+}$current. In embryos, two Shaw family genes, Shaw and Shawl, show nonoverlapping expression patterns (Hodge et al., 2005). In Drosophila, not much is known about the Shaw and Shawl channel physiological properties (Frolov et al., 2012). Studies in the blowfly Caliphora vicina identified different potassium conductances underlying LMC light responses, resulting in different processing mechanisms in different neurons (Hardie and Weckström, 1990; Rusanen and Weckström, 2016). While L2 cells display a rapidly inactivating $\mathrm{K}_{\mathrm{a}}$ type $\mathrm{K}^{+}$current, potentially mediated by the products of Shaker and Shal genes (Rusanen and Weckström, 2016; Ryglewski and Duch, 2009), L3 cells display a delayed rectifier $\mathrm{K}_{\mathrm{d}}$ type $\mathrm{K}^{+}$current, mediated by the products of $S h a b$ and Shaw genes (Rusanen and Weckström, 2016). Thus, Shaw might be important for the sustained L3 calcium responses we measure with in vivo two-photon microscopy. To test this, we performed an RNAi experiment to disrupt Shawl in L3 neurons. We then recorded calcium signals from $L 3>>$ Shawl ${ }^{\mathrm{RNAi}}$ neurons in response to full field flashes (Figure 43G) and ON and OFF flashes from intermediate gray (Figure 43H-I). During 
both stimuli presentations, L3>>ShawlRNAi neurons responses showed sustained kinetics and similar amplitude as controls. This suggests that Shawl is not required for response kinetics of L3 neurons.

Another candidate gene tested was bitesize (btsz), the only synaptotagmin-like protein in Drosophila (Pilot et al., 2006; Serano and Rubin, 2003). Its mRNA is expressed in certain epithelial tissues, for example in the salivary glands (Serano and Rubin, 2003). Btsz plays important role in multicellular epithelial tubes (Gálvez-Santisteban et al., 2012) and together with Moesin, it may mediate the proper actin organization underlying the stabilization of adherens junction (Pilot et al., 2006). We performed an RNAi experiment disrupting btsz in L3 neurons. We recorded calcium signals from $L 3>>b t s Z^{R N A i}$ neurons in response to full field flashes (Figure 43J) and ON and OFF flashes from intermediate gray (Figure 43K). When we recorded calcium responses from $L 3>>b t s Z^{R N A i}$ axon terminals we observed response kinetics to be similar as controls, meaning responses did not become transient when btsz was disrupted. In addition, statistical analysis did not show statistically significant difference between the L3>>btsZ ${ }^{R N A i}$ and control L3 neurons (Figure 43L). However, an increase in sample size might be able to pull out this difference. Despite this, btsz is not responsible for sustained L3 responses.

We next tested the functional role of genes that showed stronger expression in L2 as compared to L3. In visual systems of both vertebrates and invertebrates, interneurons regulate photoreceptor signals by feedback mechanisms (Gerschenfeld et al., 1980; Wu, 1991). Serial electron microscopy has shown that R1-6 give input to L1-L3 and amacrine cells and receive input from L2, L4, AC, Lawf and C3 cells (Meinertzhagen and Sorra, 2001; Rivera-Alba et al., 2011). It has been shown that Ih channels (or HCN channels) in amacrine cells regulate the glutamate release (Hu et al., 2015). Ih channels are voltagegated ion channels and show activation at negative potentials bellow $-50 \mathrm{mV}$ (Biel et al., 2009). When $I h$ is mutated in photoreceptors, photoreceptors cannot communicate appropriately with other neurons and this leads to rhythmical depolarization. Since Ih channels are expressed in amacrine cells, L1, L2 neurons, we thus asked if the mutation in the Ih channel in L2 leads to a change in transient and contrast-sensitive L2 responses. Previously, knockdown of Ih channels using RNAi showed reduced levels of Ih channels, indicating that the RNAi silencing worked (Hu et al., 2015). When we presented full-field flashes to the flies lacking Ih channels in L2 and recorded calcium signals from $L 2>>I h^{R N A i}$, we observed transient responses, similar to those of control L2 
neurons (Figure 43M). Again, an increase in sample size might be able to pull out some minor differences. Responses of $L 2>>I h^{R N A i}$ flies to the ON gray OFF stimulus showed a control-like contrast-sensitivity of these neurons (Figure 43N,0). Thus, Ih channels are not responsible for transient L2 responses.

The final candidate tested was a Slowpoke channel-binding protein (Slob). A voltagegated Slowpoke (Slo) is a calcium-dependent $\mathrm{K}^{+}$channel with large conductances (Adelman et al., 1992; Atkinson et al., 1991). These channels are engaged in action potential repolarization and in the release of the transmitters and its activity is controlled by Slob, a Drosophila Slo (dSlo) binding partner (Elkins and Ganetzky, 1988; Warbington et al., 1996). Slob are known to modulate the dSlo channel activation and can modulate action potential duration (Shahidullah et al., 2009). Expression of Slob mRNA and protein can be found throughout the Drosophila brain, including the optic lobe, where strong expression was detected in photoreceptors, lamina and medulla (Jaramillo et al., 2004). Thus, we decided to test whether Slob is shaping L2 calcium signals in L2 neurons. To do so, we used Slob knockdown flies generated by RNAi. We presented the Slob knockdown flies with full-field flashes (Figure 43P) and ON and OFF flashes from intermediate gray (Figure 43R,S). Again, knockdown flies showed the same amplitude of the response and similar kinetics as controls (Figure 42P-S). Thus, Slob is not required for transient response kinetics of L2 neurons.

To sum up, in this part of the study we wanted to identify candidate genes that distinguish L2 and L3 neurons. The candidate genes tested so far seem to not be required for sustained L3 or transient L2 responses. In the future, other candidate genes still have to be tested (see Discussion). 
A

ON
OFF

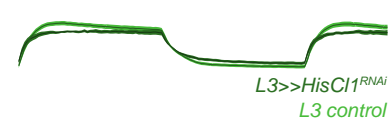

D

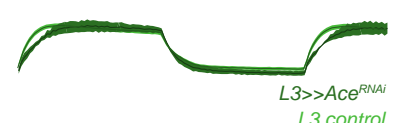

G

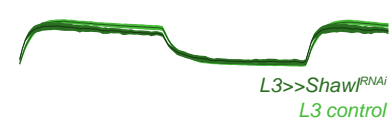

J

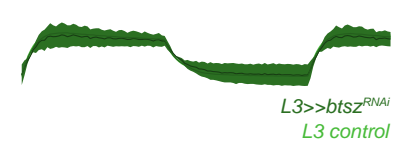

M

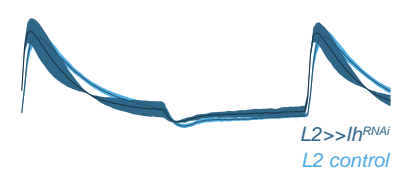

P

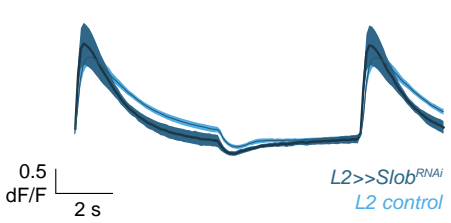

B

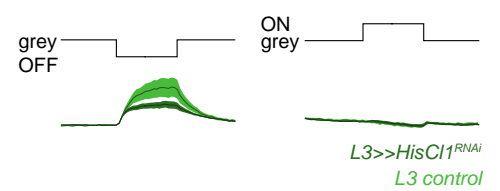

E

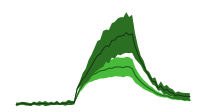

$\mathrm{H}$

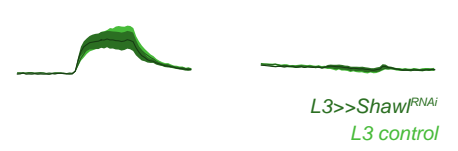

K

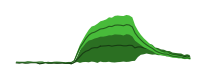

N

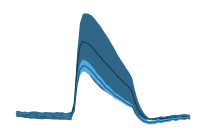

R

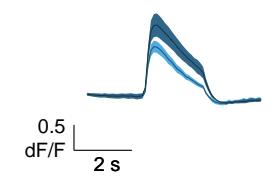

C

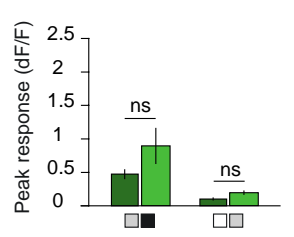

F

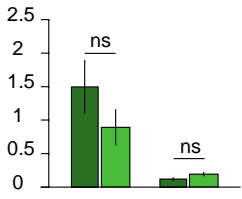

I

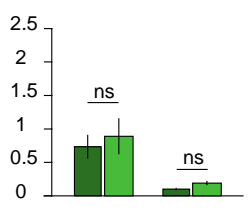

L

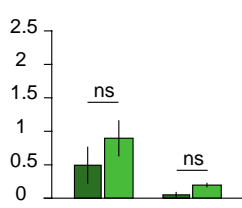

O
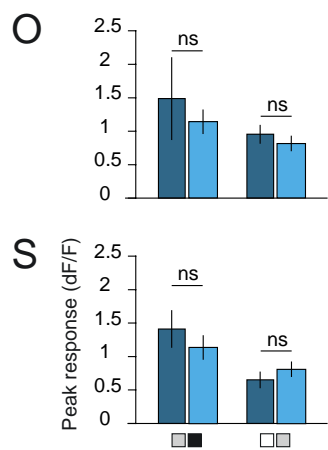

Figure 43. Testing of candidate genes via an RNAi approach.

(A,D,G,J,M,P) Average calcium responses of L3 axon terminals measured from control L3 (A,D,G,J) and L2 neurons (M,P), L3>>HisCl1fRNAi $(\mathrm{A}), L 3>>A c e^{R N A i}(\mathrm{D}), L 3>>\operatorname{Shawl}^{R N A i}(\mathrm{G}), L 3>>b t s Z^{R N A i}(\mathrm{~J}), L 2>>I h^{R N A i}(\mathrm{M})$, L2>>slobRNAi $(\mathrm{P})$ neurons to periodic $5 \mathrm{~s}$ full field flashes. $\mathrm{N}=10$ (156) for L2 controls, $\mathrm{N}=11$ (182) for L3 controls, $\mathrm{N}=7$ (76) for HisCl1fRNAi in (A), N = 3 (38) for AceRNAi in (D), N = 5 (59) for ShawlRNAi in (G), N = 2 (18) for $b t s Z^{R N A i}$ in (J), $\mathrm{N}=2$ (56) for $I h^{R N A i}$ in (M), N = 3 (58) for Slob ${ }^{R N A i}$ in (P). (B,E,H,K,N,R) Calcium signals

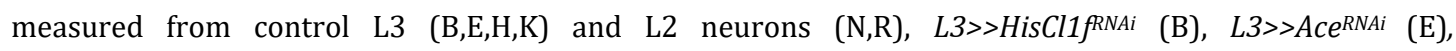
$L 3>>\operatorname{Shawl}^{R N A i}(\mathrm{H}), L 3>>b t s Z^{R N A i}(\mathrm{~K}), L 2>>h^{R N A i}(\mathrm{~N}), L 2>>\operatorname{slob}^{R N A i}(\mathrm{R})$ neurons in response to ON and OFF flashes from a gray background. (C,F,I,L, $, \mathbf{O}, \mathbf{S})$ Bar plots showing the quantification of the peak responses of control L3 (C,F,I,L) and L2 neurons (P,S), L3>>HisCl1fRNAi $(\mathrm{C}), L 3>>A c e^{\text {RNAi }}(\mathrm{F}), L 3>>\operatorname{Shawl}^{\text {RNAi }}(\mathrm{I}), L 3>>b t s Z^{\text {RNAi }}$ (L), L2>>Ih ${ }^{R N A i}(\mathrm{O}), L 2>>s l o b^{R N A i}(\mathrm{~S})$ to the two OFF steps shown in $(\mathrm{B}, \mathrm{E}, \mathrm{H}, \mathrm{K}, \mathrm{N}, \mathrm{R})$ : ON-to-gray and gray-toOFF. $* \mathrm{p}<0.05$, tested with two-tailed Student $t$ tests. $\mathrm{N}=11$ (117) for L2 controls, $\mathrm{N}=10$ (94) for L3 controls, $\mathrm{N}=7$ (125) for HisCl1fRNAi in (B,C), N = 3 (35) for Ace ${ }^{R N A i}$ in (E,F), N = 5 (76) for ShawlRAAi in (H,I), N = 3 (35) for btszRNAi in (K,L), N = 2 (62) for Ih $h^{R N A i}$ in $(\mathrm{N}, \mathrm{O}), \mathrm{N}=3$ (74) for Slob ${ }^{R N A i}$ in $(\mathrm{R}, \mathrm{S})$. 


\subsection{Integration of contrast and luminance sensitive responses in downstream circuitry}

The first step in understanding the integration of luminance information is to test whether neurons downstream of L3 still possess luminance-information. Serial EM reconstructions have revealed synaptic connections between L3 and Tm9, L3 and Mi9 and L3 and Tm20 (Takemura et al., 2013, 2017). A thorough characterization of these neurons will be necessary in order to understand how is luminance information preserved downstream of L3.

\subsubsection{Tm9 neurons receive major input from $L 3$}

Since the Tm9 neuron was found to be involved in the OFF pathway and is required for behavioral responses to motion (Fisher et al., 2015a), we decided to characterize Tm9 physiological properties. To do so, we used in vivo two-photon microscopy in which the GCaMP6f was expressed specifically in Tm9 neurons. We recorded calcium signals from Tm9 neurons in the lobula plate and presented the flies alternating full-field flashes. Consistent with L2 and L3 responses, Tm9 showed an increase in calcium signals to the light offset and a decrease to the light onset (Figure 44A-C). In contrast to L3 input, Tm9 responses showed an initial transient peak, followed by a sustained plateau response (Figure 44C). This suggests that Tm9 does not only receive and passes on the L3 signal, but instead it either takes the derivative of the L3 input signal or it integrates another, transient input.

A

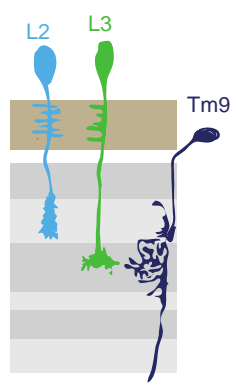

B

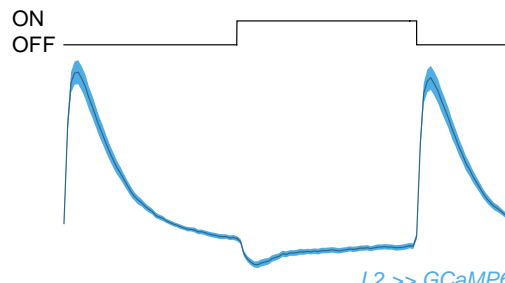

ON

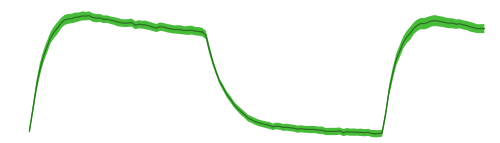

L3 >> GCaMP6f

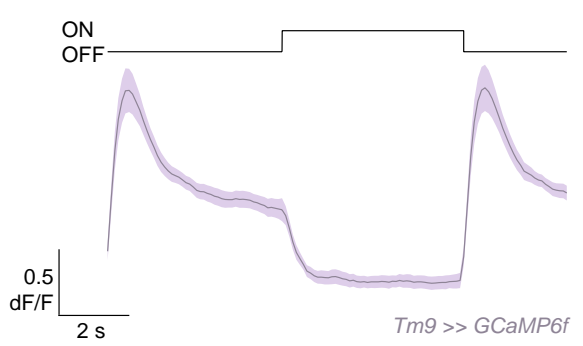

Figure 44. Tm9 responses show transient peak followed by a sustained plateau.

(A) Schematic illustrating L2 (blue), L3 (green) and Tm9 (purple) neurons. (B) Calcium signals from axon terminals in response to $5 \mathrm{~s}$ full-field flashes are shown in blue for L2, green for L3 and in purple for Tm9. N = 23(130) for $\mathrm{L} 2, \mathrm{~N}=20(224)$ for $\mathrm{L} 3, \mathrm{~N}=$ 9(141) for $\mathrm{Tm} 9$. 
To verify the functional relationship between lamina neurons and Tm9, we silenced L1, L2 or L3 and combinations of L1 plus L3 and L2 plus L3, while we imaged calcium signals from Tm9 (Figure 45A-F). We incubated flies for $1 \mathrm{~h}$ at $37^{\circ} \mathrm{C}$, which is a bit above the restrictive temperature for Shibirets, and then record Tm9 calcium signals. A statistically significant reduction of $46 \%$ in the Tm 9 transient peak response amplitude was observed when L1 neurons were silenced individually (Figure 45B). Upon L2 silencing, Tm9 responses responded with a small but non-significant decrease (Figure 44B). When L3 neurons were silenced, Tm9 responses showed a substantial reduction $(75 \%)$ in the response amplitude (Figure $45 \mathrm{C}$ ). Thus, Tm9 receives the main input from L3. However, because Tm9 responses were still present when L3 neurons were silenced, $\operatorname{Tm} 9$ has to receive at least one more input. Therefore, we blocked L3 neural activity in combination with L1 or L2 (Figure 45D-E). Silencing L3 and L2 together did not result in a stronger Tm9 phenotype than silencing L3 alone (Figure 45E). However, silencing L3 together with L1 furthermore reduced the Tm9 response amplitude by $32 \%$. Therefore, there was a $83 \%$ reduction in the Tm9 peak amplitude when L1 was silenced together with L3 (Figure 45D). Furthermore, visible responses were observed in roughly 25\% of the combinatorial L1 and L3 silenced flies, compared to more than 90\% flies of other genotypes. Only those responding flies went into subsequent analysis. This means that the mean traces shown in Figure 43 likely underestimate the strength of the phenotype of L1 + L3 silencing. Thus, L1 and L3 both provide a functional input to Tm9. Tm9 receives $\mathrm{L} 3$ input from a direct synaptic contact, while $\mathrm{Tm} 9$ most likely receives input from L1 neurons through an intermediate neuron. Since L1 is a neuron involved in an ON pathway, this result also shows that the ON and OFF pathways are not strictly separated. Additional experiments using more dynamic stimuli will be required in order to say whether the sustained Tm9 plateau is luminance sensitive as the Tm9 input, L3. However, taken together our data argue that contrast- and luminance sensitive information are combined in visual circuitry postsynaptic to lamina neurons. 
A

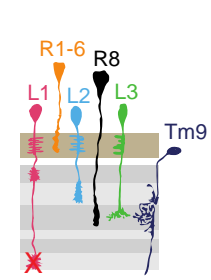

ON

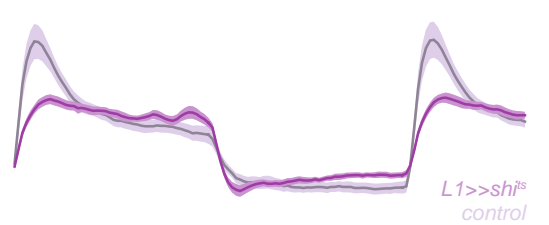

B
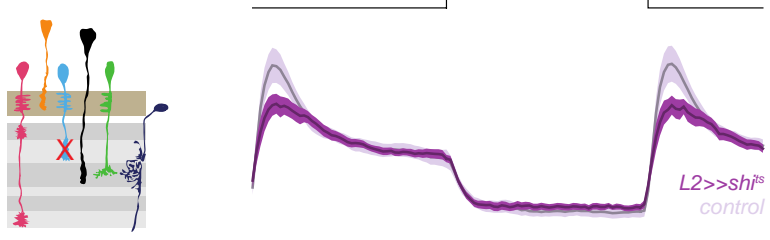

C
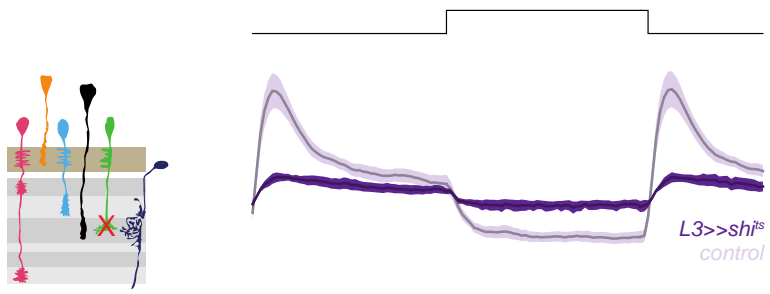

$\mathrm{D}$
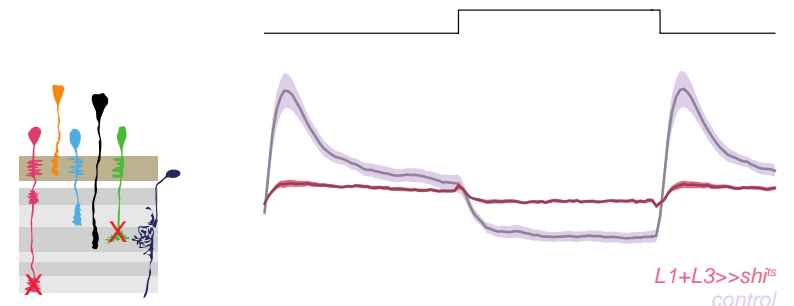

$E$

F

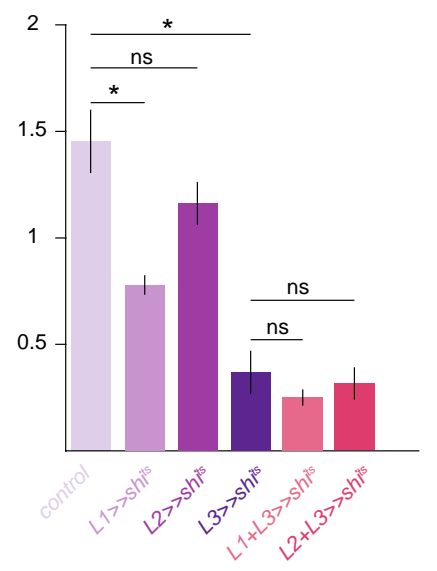

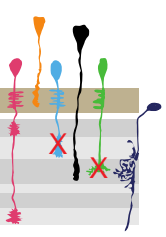

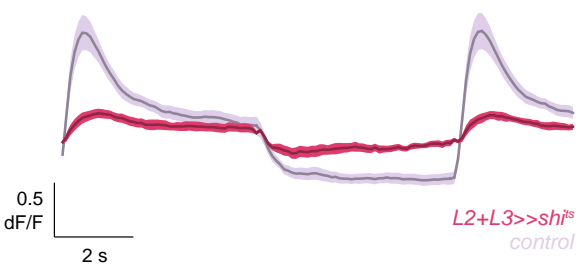

Figure 45. Tm9 integrates both L3 and L1 inputs.

(A-E) Left, schematics illustrating L2 (blue). L3 (green), L1 (red) Tm9 (purple) neurons. Red X indicates neuronal output silencing. Right, calcium signals recorded from $\mathrm{Tm} 9$ axon terminals to $5 \mathrm{~s}$ full-field flashes of control Tm9 neurons (light purple) and Tm9 neurons when either L1 (A), L2 (B), L3 (C), L1 plus L2 (D) or L2 plus L3 (E) were synaptically silenced. $\mathrm{N}=9$ (161) for TM9 control, $\mathrm{N}=10$ (188) for L1>>shits, $\mathrm{N}=12$ (171) for $L 2>>s h i t s, \mathrm{~N}=8$ (131) for $L 3>>$ shits, $\mathrm{N}=15$ (234) for $L 1+L 3>>$ shits, $\mathrm{N}=7$ (97) for $L 2+L 3>>$ shits. $^{\text {. }}$ (F) Bar plot showing the quantification of peak responses of the data shown in (A-E). ${ }^{*} \mathrm{p}<0.05$, tested using unpaired two-tailed Student's t-test. 


\section{DISCUSSION}

In this study, we demonstrate that the two OFF pathway inputs, the lamina neurons L2 and L3, are sensitive to fundamentally different features of the visual scene, contrast and luminance, respectively. The luminance-sensitive L3 pathway is mainly active in dim light, relative to the adapted state. We found that two types of photoreceptor-to-lamina transformations occur between the photoreceptors and L2 or L3. Transient and contrast-sensitive L2 neurons amplify the peak photoreceptor response and discard luminance information, whereas sustained and luminance-sensitive L3 neurons amplify photoreceptor responses in relative dim light. We investigated which mechanisms shape L2 and L3 physiological properties. Using the precise genetic tools available in Drosophila, we showed that L2 elimination of a luminance-sensitive baseline is circuitdependent, while luminance-sensitivity of L3 is regulated by the L3-specific transcription factor $d F e z f$. Together, our data demonstrate that the basic feature of the visual scene, luminance, is retained past the photoreceptor cells. Therefore, luminance information might be important for visual processing in changing light conditions.

\subsection{Contrast and luminance sensitivity of L2 and L3}

LMCs are typically described as "amplified and more transient versions of photoreceptor responses" (Laughlin, 1989), and are therefore thought to be contrast sensitive. However, it was reported before, that one LMC neuron, L3, shows physiologically distinct responses with a more sustained plateau component and a slower temporal filter than L1 and L2 (Clark et al., 2011; Rusanen and Weckström, 2016; Silies et al., 2013; Uusitalo et al., 1995). We have discovered that L3 sustained responses are luminance-sensitive. Thus, for the first time, it has been shown that luminance information is retained past the photoreceptor cells in insect eyes.

\subsubsection{Luminance information is retained past photoreceptor cells}

In our study we have found that in fruit fly the visual system, the luminance information is retained in parallel to the contrast pathway. The majority of visual systems are able to perform well in different light conditions. This is true because visual systems ignore background illumination by computing contrast. In both vertebrates and invertebrates, photoreceptors adapt to the mean illumination in order to compute contrast irrespective of the background illumination (e.g. Clark and Demb, 2016; Laughlin and Hardie, 1978; Normann and Werblin, 1974). However, photoreceptor adaptation is not instantaneous. Instead, photoreceptor adaptation has a fast component, occurring within tens of milliseconds, as well as a slow component, lasting for tens of seconds (e.g. 
Adelson, 1982; Dowling and Ripps, 1972; Laughlin, 1989; Laughlin and Hardie, 1978). Therefore, photoreceptor adaptation is sufficient to correctly compute contrast when the luminance changes are slow, e.g. during daylight and dusk or dawn. On the other hand, adaptation time scales may be slower than the rapid environmental conditions encountered by the animal's own movement. Human eyes also constantly experience rapid luminance changes by saccadic eye movements and require quick adjustments in sensitivity in order to guide appropriate behavioral responses (e.g. Rieke and Rudd, 2009). Rapid changes in visual statistics, which are faster than the photoreceptor adaptation time scales, could lead to a misinterpretation of visual cues leading to an inappropriate behavioral response of an animal. Therefore, we suggest that luminance information might facilitate visual processing under such dynamic conditions and thus aid image processing at conditions that are challenging for purely contrast-sensitive pathways.

I have showed that a luminance-sensitive pathway is mainly active in relative dim light. Matching this physiological specialization, behavioral experiments confirmed that this luminance-sensitive pathway is required for appropriate behavioral responses to motion in relative dim light, when background illumination is constantly changing (Ketkar and Silies, unpublished). Without the luminance information, the flies had deficits in motion detection in relative dim light, indicating that the L3 pathway is required when pure contrast sensitivity underestimates the salience of a stimulus. We suggest that a luminance-sensitive signal is used to scale the contrast-sensitive response for dim stimuli, in order to guide appropriate behavioral responses. Since adaptation time scales between invertebrates and vertebrates are very similar, this might be a general image processing strategy across the animal kingdom.

\subsubsection{Phasic and tonic responses are present in different sensory systems}

L2 and L3 show different responses to sustained input, with L2 having transient or phasic and L3 sustained or tonic responses. Phasic responses are rapidly adapting and report stimulus changes, whereas tonic receptors are slowly or non-adapting and report the amplitude of the signal. Previous electrophysiological recordings from the optic chiasm between lamina and medulla in flies already showed phasic and tonic units (Arnett, 1972; Mimura, 1974). Besides that, recordings in the medulla region of different insect species also reported these two response types (Glantz, 1973; Honegger, 1978). In addition, phasic and tonic responses have been observed in neurons with graded as well as action potentials, in different sensory systems, and in many different species (Erickson, 2013). Premotor and motor cortex neurons in the monkey show phasic and tonic responses to the torque step perturbation of the wrist (Bauswein et al., 1991). 
Phasic, tonic and intermediate neuron population have been described in toad olfactory epithelium in response to sustained depolarizing current stimuli. Examples from Drosophila include proprioceptive sensory neurons located in the femoral chordotonal organ, which can also be classified either as tonic and encoding position, or phasic and encoding movement (Mamiya et al., 2018). Besides that, neurons in the Johnston's organ exhibit either phasic or tonic responses, depending on the magnitude and type of aristae displacement (Kamikouchi et al., 2009; Yorozu et al., 2009).

Taken together, the presence of both tonic and phasic neurons in different sensory systems appears ubiquitous, and tonic and phasic responses provide distinct information. It will be interesting to see if the mechanisms identified here also differentiate tonic and phasic responses in other systems. For example, one could test if the transcription factor dFezf or its vertebrate homologues Fezf1 or Fezf2 are also expressed in phasic neurons of other sensory systems. Our work suggests that tonic and phasic responses must be combined in downstream circuits in the fly visual system in order to drive appropriate responses. It will be further interesting to see if this is true in other sensory systems as well. To the best of my knowledge, exact mechanisms of how the tonic and phasic information are integrated in the downstream circuits have not yet been identified. However, it appears plausible that the presence of the absolute value of peripheral stimulus might also be used in other sensory systems to modulate perception of the change in stimulus detected by other pathways. Thus, retaining a peripheral sensory feature in higher order processing might be a general sensory processing strategy.

\subsubsection{A role for luminance in image processing might be an evolutionary conserved feature}

In this study we showed that the L3 responses carry luminance information. Previously, some studies have shown the presence of luminance information in the retina or the brain of vertebrates and invertebrates. It has been suggested that animals use eyes to form images from spatiotemporally patterned light information, whereas slowly changing illumination is detected by additional regions of the body, i.e. pineal organ (Currie et al., 2016; Ekström and Meissl, 1990). Intrinsically light sensitive neurons can cover a broad luminance range and are engaged in the circadian clock regulation, sleep control and hormones (Berson et al., 2002; Milner and Do, 2017; Vinayak et al., 2013). Presence of luminance information has also been shown in the brain of Xenopus laevis frog tadpoles, where their swimming activity is linked by the ambient light levels (Currie et al., 2016). Higher up in the visual processing, certain cat and primate V1 neurons were shown to be sensitive to illumination (DeYoe and Bartlett, 1980; Kayama et al., 
1979). The so called "luxotonic" neurons have been described previously (DeYoe and Bartlett, 1980; Kayama et al., 1979), which exhibit sustained firing rate as a result of changes in the ambient light level. Together, slowly changing luminance information is important for the normal circadian clock (Berson et al., 2002; Nippe et al., 2017).

While all of these systems signal really slow light changes, in our study we show that luminance information associated with faster changes is retained in the Drosophila visual system downstream of the photoreceptor cells. This is the case also in vertebrates, where the luminance information was found to be present in the retina. There, a luminance-sensitive response component is retained past photoreceptors at the rod bipolar cell (RBC) to amacrine cell (AII) synapse (Odermatt et al., 2012; Oesch and Diamond, 2011). Synaptic release from these synapses showed an adaptation to luminance increases and therefore generated a transient response component encoding contrast in the AII. Besides that, the AII sustained component represents the RBC response and thus carries luminance information. Interestingly, L2 and L3 calcium signals look very similar to calcium signals recorded from specific bipolar cell classes in the vertebrate retina (Baden et al., 2013). There, bipolar cells differ in their kinetics and range from sustained, to slowly decaying and transient and the bipolar cell output can be directly reflected in the layered anatomical organization (Baden et al., 2013; Borghuis et al., 2014; Euler et al., 2014). This, together with the behavioral relevance of luminance information discussed above, argues that information about fast luminance changes retained in the retina might be needed to aid image processing across animal species.

\subsubsection{Luminance information as an advantage for animals living in certain environments}

Vision in very dim light can be challenging due to the randomness of rare photon arrivals. Despite this, many animal species can see remarkably well at extremely low luminances (Aho et al., 1988; Stöckl et al., 2016; Warrant et al., 2004). Many nocturnal insects and crustaceans, such as nocturnal moths, beetles and deep-sea crustaceans possess superposition compound eyes (Land, 1990; Warrant, 2017; Warrant and McIntyre, 1993). In addition, different insects with simple apposition eyes have evolved and can thus perform very well in darkness. To do so, the sensitivity of their eyes is improved by a photon summation, resulting in motion-sensitive neurons, which can perform better than photoreceptors responses would predict (e.g. O'Carroll and Warrant, 2017; Stöckl et al., 2016; Warrant et al., 2004). Therefore, evolution has allowed nocturnal animals to well adapt to their living conditions, however these animals might still require a luminance-sensitive pathway in order to tackle challenges posed by extremely low light levels. 
However, we found that the luminance-sensitive L3 neuron is mainly active in dim light relative to different adaptation states. This could suggest that the requirement for a luminance-sensitive pathway is higher in animals living in areas with rapid and broad luminance changes. Diurnal animals navigating through their environment on a sunny day encounter big luminance changes. For example, when a fly is navigating through its environment, it might encounter a predator. Due to the adaptation of the visual system, a fly will equally well detect contrasts elicited by the dragonfly at different daylight conditions and therefore escape the predator (Figure 46). But a problem might appear when the predator is hiding under the shade of a leaf. In this case, where a change in background intensity is close to instantaneous, the visual system might not be able to adjust light sensitivity equally fast and underestimate contrast. This would lead to a very small response of contrast-sensitive neurons, which would underestimate the salience of the stimulus, leading to an insufficient behavioral response (Figure 46). Therefore, diurnal animals that encounter big luminance changes more likely possess a luminancesensitive channel. The presence of luminance-sensitive pathway might help to overcome the limitations of adaptation in order to ensure accurate motion detection when encountering a rapid dim light (Figure 46). In conclusion, diurnal and nocturnal animals might both possess luminance-sensitive pathway, however, they might use this luminance information in different ways.
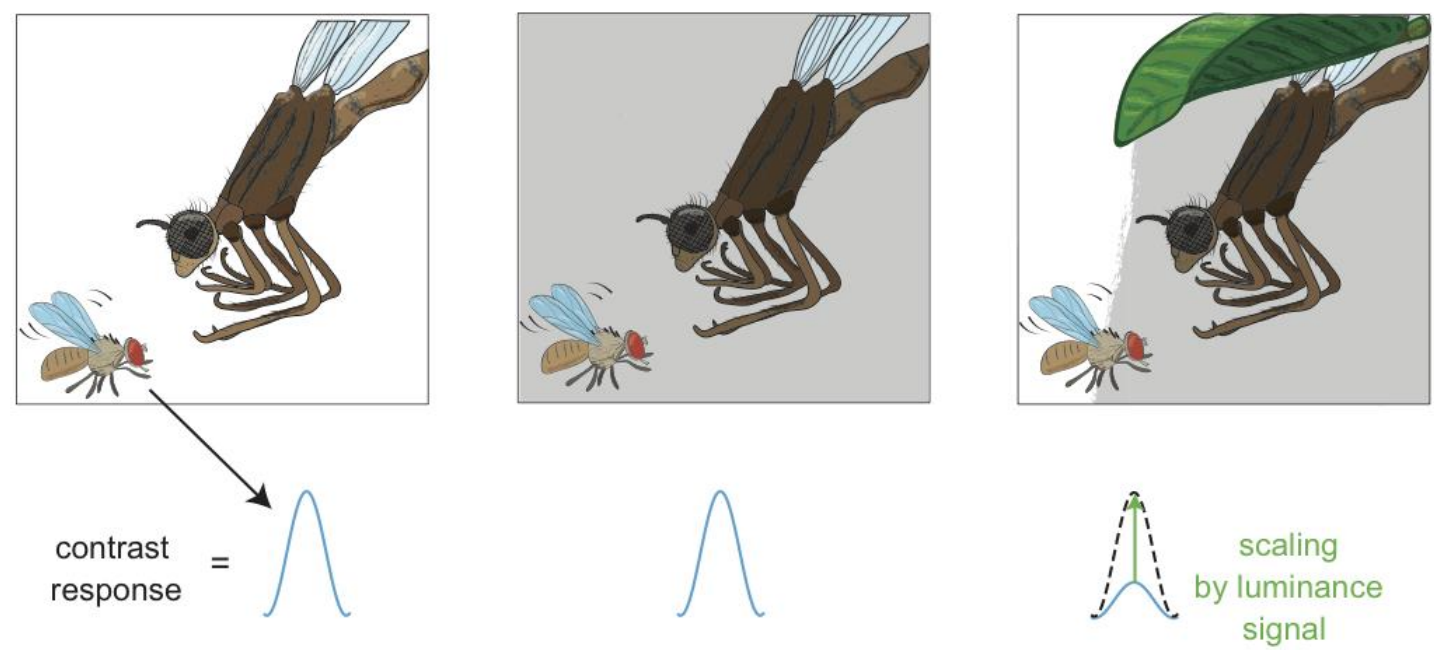

Figure 46. Luminance information scales the contrast-sensitive response.

Schematic illustrating a moving fly and a dragonfly on a bright background (left), a dark background (middle), or a background of changing luminance (right). Below, schematic of a predicted neuronal or behavioral response to contrast. At slowly changing background light intensities such as changing daylight, adaptation mechanisms are sufficient to produce appropriate contrast responses under varying conditions. Under fast changing light conditions, contrast-sensitive neurons might underestimate the salience of a visual stimulus. A luminance-sensitive neuron, e.g. L3, could scale a contrast-sensitive response to produce appropriate behavior. 
In vertebrates, intrinsically photosensitive retinal ganglion cells (ipRGCs), are engaged in the circadian rhythm regulation (Berson et al., 2002; Hattar et al., 2002; Pickard and Sollars, 2012). In Drosophila, circadian rhythm is controlled by the central pacemaker and peripheral clocks, one of which is located in the retina and partially in the lamina (Damulewicz et al., 2013, 2015).

Therefore, it would be exciting to see whether the luminance-sensitive L3 neuron might also contribute to slower luminance-sensing mechanisms, such as the circadian clock. In addition, it would be interesting to see whether other insect species have evolved similar luminance-sensitive pathway as we discovered in Drosophila. One could check whether closely related insect species show the same luminance-sensitivity regardless of their different ecological niches. Some differences in the behavior and visual response properties as well as in the eye design have been described. For example, $D$. melanogaster is a generalist, which can be found in urban environments and shows repulsive responses to small visual stimuli. In contrast, Drosophila mojavensis is a specialist, which can be found in desert landscapes and shows attractive responses to small visual stimuli (Park and Wasserman, 2018). Besides that, it was reported that, differences in the eyes have been identified between D. mauritiana and D. simulans (Posnien et al., 2012). Therefore, one should test contrast and luminance sensitivity of different Drosophila species in order to see whether closely related species, but living in different environments, possess similar strategy of the early visual processing.

\subsection{Mechanisms that shape L2 and L3 physiology}

In our study we showed that L2 and L3 get the main photoreceptor input from R1-R6. However, two different types of photoreceptor-to-lamina transformations occur between R1-R6 and L2 or L3. We showed that L2 become contrast sensitive by a circuitdependent elimination of baseline, whereas L3 luminance sensitivity involves a single, L3-specific transcription factor.

\subsubsection{Sustained R1-R6 calcium responses are sufficient for normal L2 and L3} responses

Calcium signals recorded from R1-R6 captured the steady-state component of the photoreceptor response that displayed luminance sensitivity. Voltage recording from photoreceptors have shown that the photoreceptor cells respond to the prolonged illumination with an initial transient phase, which encodes contrast, followed by a plateau phase, which is luminance-sensitive. This initial transient peak was missed in 
calcium recordings. There might be a few reasons for this discrepancy. First, from voltage recording we know that photoreceptors respond with a sustained response without the initial transient peak in response to flashes of intermediate intensities (see Figure 4B). Thus, we might be imaging in a light regime, which is not bright enough to produce this initial transient response. Second, genetically encoded calcium indicator might not be fast enough to detect this rapid transient phase of the photoreceptor response. However, the detection rate of the GCaMP6f is tens of milliseconds (Chen et al., 2013) and the initial transient photoreceptor response lasts for 100-200 milliseconds (e.g. Laughlin and Hardie, 1978).

We showed that both L2 and L3 obtain the main input from R1-R6. We used two different approaches to test the potential different photoreceptor contribution to L2 and L3. While both approaches agree that the predominant input into L2 is coming from R1R6, there were some discrepancies whether R8 provides input to L2 neurons or not. In the first approach to test the specific R8 contribution we used a ninaE mutant background, making R7 and R8 the only functional photoreceptors. This experiment suggested that R8 alone does not provide any functional input to L2. In contrast, norpA experiment showed that R8 might provide some input from R8. This input is indirect, as there are no direct synapses observed between R8 and L2 (Takemura et al., 2013). The discrepancies between the two approaches might be because of two reasons. First, ninaE mutation can cause structural deterioration of inner and outer photoreceptors. The deterioration is gradual and takes a few weeks, with R1-R6 degenerating first, followed by R7 and R8 (Leonard et al., 1992). Flies used in our experiment were only a few days old therefore the deterioration might be rather small. Second, in the norpA approach we used norpA overexpression. In normal conditions, genes are expressed at the appropriate levels and a reduction of expression of any gene below a critical threshold will result in a mutant phenotype. However, an increased expression of a wild-type gene can also change normal cellular properties (Prelich, 2012). Thus, norpA overexpression could cause a stronger R8 input to L2 neurons and therefore generating an above physiological response in L2 neurons when only R8 are active. Despite this different result, it is safe to conclude that although R8 seem to provide input to both L2 and L3, the contribution is rather small. The complementary experiment further confirmed that R1-R6 are sufficient to restore normal L2 and L3 responses.

\subsubsection{L2 elimination of a baseline is circuit-dependent}

L2 and L3 contrast and luminance sensitivity arise from distinct cellular and circuit properties. Our data show that the elimination of a luminance-sensitive baseline in L2 is not established at the photoreceptor-to-L2 synapse, but instead it is a result of a circuit 
mechanism. However, the exact circuit mechanism is yet to be discovered. We propose an inhibitory candidate, which also receives photoreceptor input and can therefore subtract the L2 baseline. A candidate that fits this criterion is the L3 neuron itself, as it receives direct input from the outer photoreceptors and possesses the right physiological properties. However, there are no synapses between L2 and L3 neurons and L3 is thought to be cholinergic and thus excitatory (Meinertzhagen and O'Neil, 1991b; Meinertzhagen and Sorra, 2001). Other potential candidates could be outer photoreceptors themselves or L1 neurons, which are glutamatergic and thus can act as either inhibitory or excitatory inputs (Liu and Wilson, 2013; Richter et al., 2018). However, L1 also does not provide direct input onto L2, making inhibitory interneurons a likely candidate. Based on connectomics, L2 obtaines a strong input from the GABAergic C3 and C2 columnar feedback neurons (Kolodziejczyk et al., 2008; Meinertzhagen and O'Neil, 1991b). Furthermore, both C2 and C3 receive dominant input from L1 (Takemura et al., 2013). We have shown that silencing L1 neurons together with L3 leads to diminished Tm9 responses, suggesting a role of the ON neuron in the OFF pathway (Fisher et al., 2015a; Silies et al., 2013). This result supports the idea of C2 and C3 as candidate neurons as they receive an ON pathway input from L1 and feed into the OFF pathway, L2. Thus, both C2 and C3 are promising candidates to eliminate the L2 baseline. Besides that, little is known about inhibitory amacrine-like Dm neurons (Nern et al., 2015) and their connectivity is unknown. Thus, these neurons might be further candidates in eliminating the L2 baseline. In insects, GABA and glutamate serve as inhibitory neurotransmitters. One could first broadly test the role of inhibition and distinguish between these two neurotransmitter systems. This can be achieved using pharmacology combined with in vivo two-photon calcium imaging. It is known that picrotoxin blocks $\mathrm{GABA}_{\mathrm{A}} \mathrm{R}$ at low concentrations, whereas at higher concentrations it blocks the glutamate gated chloride channels GluClalpha (Cleland, 1996; Fisher et al., 2015b; Liu and Wilson, 2013). Besides that, one could test candidate neurons that shape L2 responses by genetically silencing them using shibire ${ }^{\text {ts }}$ while recording from L2. This simultaneous calcium recording in one cell type and silencing activity in the other cell type can be achieved by using two different binary expression systems, the Gal4-UAS and the LexA-lexAop system. Doing so we could test the role of GABAergic C2 and C3 neurons or GABAergic or glutamatergic amacrine-like Dm neurons in shaping L2 responses (Fischbach and Dittrich, 1989; Nern et al., 2015). 


\subsubsection{Luminance-sensitivity of L3 is regulated by a single L3-specific} transcription factor

L3 inherits distinct physiological properties from photoreceteptors that are very different to L2 properties. While such differences can in principle arise presynaptically or postsynaptically, R1-R6 synapse onto both L2 and L3 lamina neuron types within one tetrad synapse (Frohlich and Meinertzhagen, 1982). Different presynaptic sites from a single neuron, e.g. a photoreceptor cell in our case, could still evoke different synaptic responses in postsynaptic cells, e.g. L3 or L2 neurons. However, we showed that L3 neuronal response properties are established by cell-autonomous mechanisms involving transcriptional targets of dFezf and therefore must be established at the postsynaptic site. L3 dFezf mutant neurons become transient and contrast sensitive, resembling wild-type L2 responses. $d F e z f$ was previously identified as a transcription factor required for appropriate L3 axon terminal targeting. Without $d F e z f$, L3 axon terminals do not project to the M3 layer of the medulla, but instead they project to M1 or M2 (Peng et al., 2018). Furthermore, L3 dFezf mutant neurons display defects in axon terminal morphology. Wild-type L3 neurons are easy to distinguish among other LMCs due to a unique dendritic tree in the lamina, as all L3 dendrites project to one side (Fischbach and Dittrich, 1989). L3 dFezf mutant neurons had normal dendritic processes and early in development they still expressed L3-specific cell markers. Later on, in L3 $d F e z f$ mutant neurons, certain L3-specific genes become downregulated whereas certain genes that are otherwise normally expressed in other lamina neurons become upregulated (Peng et al., 2018). However, L3 dFezf mutant neurons do not turn on L2specific cell markers. Therefore, dFezf disruption does not lead to an obvious cell fate change (Peng et al., 2018). Overexpression of $d F e z f$ in L2 neurons mediates mistargeting to the M3 layer (Peng et al., 2018). In contrast, L2>>dFezf neurons show similar response kinetics as wild-type L2 neurons, indicating that dFezf is sufficient for the targeting phenotypes. Together, dFezf is necessary but not sufficient to establish L3specific response properties. Together this argues that $d F e z f$ has separate roles in determining axon targeting and L3-specific physiological properties. In addition, silencing the output of L3 did not cause a change in L3 response kinetics. This further suggests that L3 responses properties are not influenced by the synaptic outputs in its target layer.

Our data argue that L3 specific properties are established on the postsynaptic site in L3, and that transcriptional targets of dFezf mediate L3 neuronal response properties. To identify the gene targets downstream of $d F e z f$ that mediate L3-specific properties, one 
could perform RNAseq experiments from adult wild-type L2 and L3 neurons as well as from L2 neurons overexpressing dFezf and from L3 MARCM clones mutant for dFezf. This should be able to result in a list of the candidates that could mediate physiological properties, but also axon targeting in L3. Since dFezf is sufficient for growth cone targeting in M3 layer (Peng et al., 2018), but not L3 physiology, one should not first concentrate on genes that are only differentially regulated between controls and L3 $d F e z f$ loss of function. However, it might be possible that there are some homeostatic mechanisms shaping L2>>dFezf responses preventing us to see a physiological phenotype upon $d F e z f$ overexpression in L2. Therefore, one could subsequently take into consideration all differentially expressed genes between L2 control and L2>>dFezf in order to find the gene targets downstream of dFezf that mediate L3-specific properties. In a complementary approach it is possible to map transcriptional targets of dFezf, using ChipSeq (Akhtar et al., 2019). Similar as in the RNAi screen approach, we should then focus on the candidate genes, which are likely to change the cell biophysical membrane properties, e.g. channels, receptors and transporters. Candidate genes should then be functionally validated by loss of function experiments.

Complementary to the candidate-based approach where we identified dFezf as an important regulator of L3 function, we also started with a preliminary RNAi screen in order to identify candidate genes that distinguish L2 and L3 physiological properties. The candidate genes tested so far seem to not be required for sustained L3 or transient L2 responses. It is possible that the RNAi silencing was ineffective or produced a strong hypomorphic situation. However, the stimuli used in these experiments might not have been suitable to show a strong phenotype upon candidate gene silencing. Instead, one should use more sophisticated stimuli such as "contrast steps from adapted background" or "flashes of different intensity", to specifically probe for contrast- and luminance sensitivity.

Another thing worth trying would be strengthening of the RNAi phenotype. There are several ways to do so. First, one could grow the flies at higher temperatures. Flies used in our study were incubated at $25^{\circ} \mathrm{C}$ and the expression strength of Gal4 is temperature sensitive, thus one could try enhancing the RNAi effect by growing the flies at $29^{\circ} \mathrm{C}$. Second, another way to strengthen the RNAi effect would be by expressing Dicer-2 (Dcr2). RNAi is a gene-silencing process induced by double-stranded RNA (dsRNA) (Hannon, 2002). dsRNA can be processed by the dsRNA-specific endonuclease Dicer, resulting in small interfering RNA (siRNA) or microRNA (miRNA). In Drosophila, two different Dicer 
(Dcr) enzymes are found: Dcr-1, which generates miRNA and Dcr-2, which generates siRNA (Lee et al., 2004; Liu et al., 2003). Expression of Dcr-2 can enhance the RNAi effect (Dietzl et al., 2007), however, it can also cause non-specific phenotypes due to off-target effects (Kulkarni et al., 2006; Ma et al., 2006). In addition, if Gal4 is used for driving both RNAi and GCaMP expression, it might result in a diluted knockdown phenotype. To circumvent this problem, one could also try using two different expression systems, e.g. LexA-lexAop for GCaMP6f expression and UAS-Gal4 for the RNAi expression. Complementary to the sufficiency tests, one could perform overexpression experiments to test whether the candidate genes differentially expressed in L2 and L3 are sufficient for sustained or transient responses.

\subsection{Integration of luminance and contrast information downstream of the lamina}

We showed that L3 is luminance-sensitive and that the luminance sensitivity is important for motion detection in dim light. We next wanted to know how luminance and contrast information are being combined in order to control motion-guided behaviors. Calcium recordings from a neuron downstream of L3, Tm9, showed that calcium signals in $\mathrm{Tm} 9$ are different to those from L3, and showed an initial transient peak, followed by a sustained plateau. This can be achieved by taking the derivative of the L3 input signal or by integration of another input with transient kinetics. We showed that $\mathrm{Tm} 9$ receives additional input from another lamina neuron, the biphasic $\mathrm{ON}$ pathway input neuron L1. When L1 output is blocked, Tm9 neurons did not show a pronounced initial transient response anymore, whereas the sustained component of the response remains. This furthermore argues that the luminance information is combined with contrast information at the level of the medulla neurons. Other medulla neurons possess either transient or sustained responses. In the ON pathway, Mi1 and Tm3 show transient responses, whereas Mi9 show sustained responses to a sustained visual input (Arenz et al., 2017; Behnia et al., 2014; Strother et al., 2017). Interestingly, the sustained Mi9 neuron receives the main input from the OFF pathway neuron L3, and then provides inhibitory input onto the ON pathway at the level of the T4 (Takemura et al., 2013, 2017). This could suggest that L3 neurons provide luminance information to both ON and OFF pathways. In addition, L3 makes synapses with the Mi1 neuron, which receives the main input from the L1 neuron (Takemura et al., 2013). This suggests that medulla neurons downstream of L3 show transient responses to flashes if they receive input from the ON pathway also, and sustained if their main input neuron is L3. Neurons postsynaptic to the other OFF pathway neuron, L2, all have transient responses kinetics 
(Behnia et al., 2014; Meier et al., 2014; Serbe et al., 2016; Takemura et al., 2013; Yang et al., 2016). Transient or sustained medulla cells project onto T4 and T5 cells suggesting that luminance and contrast information can be further integrated in the T4 and T5 dendrites. It was shown that models of motion computation with added tonic or DC component perform better than models without it (Eichner et al., 2011; Leonhardt et al., 2017) suggesting an importance of luminance information in motion computations. Till now, it was not known how this tonic component is generated and propagated through the circuits. We have discovered a pathway that can provide such a DC signal, arguing that the DC component most likely originates from L3, however the exact integration of this luminance information is yet to be discovered. 


\subsection{Outlook}

In the course of this study on fly visual processing, we showed that information about the most fundamental visual feature, luminance, is preserved beyond the photoreceptor cells. We showed that L2 and L3, the two first order lamina interneurons, respond to different features of the visual scene, contrast and luminance, respectively. We have identified the coarse mechanisms that shape L2 and L3 physiological responses, however, the precise circuit and exact genes are still yet to be discovered. In order to further understand the circuit subtraction of L2 baseline, one should silence the output of candidate neurons, which provide inputs to L2 neurons based on connectomics, and record calcium responses from L2 neurons. In order to identify the genes shaping L3 responses, mapping the transcriptional targets of dFezf, followed by RNAi silencing of candidate genes will be necessary.

Unpublished work from the lab has shown that contrast-sensitive and luminancesensitive pathways interact to guide behavior. Therefore, one should try to understand the mechanisms of the interaction between contrast- and luminance-sensitive pathways, which enable appropriate behavioral responses to motion under challenging light conditions. Recordings from downstream neurons might give us answers to how are the contrast and luminance information integrated in downstream circuits. More dynamic stimuli than full-field flashes can also be used to test the luminance and contrast sensitivity of downstream neurons such as Tm9.

It would be exciting to know how the above-mentioned findings differ across different Drosophila species and across different insect species living in different ecological niches. Genetically encoded calcium indicators are currently being developed for use in other Drosophila species. Bath-applied calcium-sensitive dyes have been used in insects, however they are not cell-type specific and are, due to the long incubation time, not very appropriate for small insect brain such as Drosophila. It is also possible to characterize the visual response properties of different insect species using behavioral experiments. One could compare visual responses of fly species from different ecological niches, flies active at different times of the day and flies with different anatomy of their compound eye. This could reveal where luminance information is required and whether it plays a similar role in different insect species. Since visual processing in vertebrates shares many common features with invertebrates, it would be interesting to see if the presence of luminance-sensitive signals in the vertebrate retina is also used to scale contrastsensitive responses under specific light conditions. Our finding that the fly visual system 
maintains luminance in parallel to contrast information and uses it to correctly estimate the salience of a visual stimulus may reveal a general image processing strategy. 


\section{BIBLIOGRAPHY}

Abrahamson, E.W., Fager, R.S., and Mason, W.T. (1974). Comparative properties of vertebrate and invertebrate photoreceptors. Exp. Eye Res. 18, 51-67.

Adelman, J.P., Shen, K.Z., Kavanaugh, M.P., Warren, R.A., Wu, Y.N., Lagrutta, A., Bond, C.T., and Alan North, R. (1992). Calcium-activated potassium channels expressed from cloned complementary DNAs. Neuron 9, 209-216.

Adelson, E.H. (1982). Saturation and adaptation in the rod system. Vision Res. 22, 1299-1312.

Adelson, E.H., and Bergen, J.R. (1985). Spatiotemporal energy models for the perception of motion. J. Opt. Soc. Am. A. 2, 284-299.

Aho, A.C., Donner, K., Hydén, C., Larsen, L.O., and Reuter, T. (1988). Low retinal noise in animals with low body temperature allows high visual sensitivity. Nature 334, 348-350.

Ainsworth, M., Lee, S., Cunningham, M.O., Traub, R.D., Kopell, N.J., and Whittington, M.A. (2012). Rates and Rhythms: A Synergistic View of Frequency and Temporal Coding in Neuronal Networks. Neuron 75, 572-583.

Akhtar, J., More, P., Kulkarni, A., Marini, F., Kaiser, W., and Berger, C. (2019). TAF-ChIP: An ultralow input approach for genome wide chromatin immunoprecipitation assay. BioRxiv 299727.

Aldridge, W.N. (1950). Some properties of specific cholinesterase with particular reference to the mechanism of inhibition by diethyl p -nitrophenyl thiophosphate (E 605) and analogues. Biochem. J. 46, 451-460.

Arenz, A., Drews, M.S., Richter, F.G., Ammer, G., and Borst, A. (2017). The Temporal Tuning of the Drosophila Motion Detectors Is Determined by the Dynamics of Their Input Elements. Curr. Biol. 27, 929-944.

Arnett, D.W. (1972). Spatial and temporal integration properties of units in first optic ganglion of dipterans. J. Neurophysiol. 35, 429-444.

Ashburner, M. (1989). Drosophila: A Laboratory Handbook (Cold Spring Harbour: Cold Spring Harbor Laboratory Press).

Asteriti, S., Liu, C.H., and Hardie, R.C. (2017). Calcium signalling in Drosophila photoreceptors measured with GCaMP6f. Cell Calcium 65, 40-51.

Atkinson, N.S., Robertson, G.A., and Ganetzky, B. (1991). A component of calcium-activated potassium channels encoded by the Drosophila slo locus. Science (80-. ). 253, 551-555.

Baden, T., Schubert, T., Chang, L., Wei, T., Zaichuk, M., Wissinger, B., and Euler, T. (2013). A tale of two retinal domains: Near-Optimal sampling of achromatic contrasts in natural scenes through asymmetric photoreceptor distribution. Neuron 80, 1206-1217.

Bader, C. (1976). Role of intracellular calcium and sodium in light adaptation in the retina of the honey bee drone (Apis mellifera, L). J. Gen. Physiol. 67, 475-491.

Baines, R.A., Uhler, J.P., Thompson, A., Sweeney, S.T., and Bate, M. (2001). Altered electrical properties in Drosophila neurons developing without synaptic transmission. J. Neurosci. $21,1523-1531$.

Bardell, D. (1993). Hodierna's “The Eye of the Fly”: The First Book of Microscopic Observations. Bioscience.

Barlow, H.B., and Levick, W.R. (1965). The mechanism of directionally selective units in rabbit's retina. J. Physiol. 178, 477-504.

Bauswein, E., Fromm, C., Werner, W., and Ziemann, U. (1991). Phasic and tonic responses of premotor and primary motor cortex neurons to torque changes. Exp. Brain Res. 86, 303310.

Behnia, R., Clark, D.A., Carter, A.G., Clandinin, T.R., and Desplan, C. (2014). Processing properties of ON and OFF pathways for Drosophila motion detection. Nature 512, 427-430.

Belušič, G., Ilić, M., Meglič, A., and Pirih, P. (2016). A fast multispectral light synthesiser based on LEDs and a diffraction grating. Sci. Rep. 6, 32012.

Bender, W., Spierer, P., Hogness, D.S., and Chambon, P. (1983). Chromosomal walking and jumping to isolate DNA from the Ace and rosy loci and the bithorax complex in 
Drosophila melanogaster. J. Mol. Biol. 168, 17-33.

Bernstein, E., Caudy, A.A., Hammond, S.M., and Hannon, G.J. (2001). Role for a bidentate ribonuclease in the initiation step of RNA interference. Nature 409, 363-366.

Berson, D.M., Dunn, F.A., and Takao, M. (2002). Phototransduction by retinal ganglion cells that set the circadian clock. Science (80-. ). 295, 1070-1073.

Biel, M., Wahl-Schott, C., Michalakis, S., and Zong, X. (2009). Hyperpolarization-Activated Cation Channels: From Genes to Function. Physiol. Rev. 89, 847-885.

Blakemore, C., and Campbell, F.W. (1969). On the existence of neurones in the human visual system selectively sensitive to the orientation and size of retinal images. J. Physiol. 203, 237-260.

Van Der Bliek, A.M., and Meyerowrtz, E.M. (1991). Dynamin-like protein encoded by the Drosophila shibire gene associated with vesicular traffic. Nature 351, 411-414.

Borghuis, B.G., Looger, L.L., Tomita, S., and Demb, J.B. (2014). Kainate Receptors Mediate Signaling in Both Transient and Sustained OFF Bipolar Cell Pathways in Mouse Retina. J. Neurosci. 34, 6128-6139.

Borst, A. (2009). Drosophila's View on Insect Vision. Curr. Biol. 19.

Borst, A., and Haag, J. (2002). Neural networks in the cockpit of the fly. J. Comp. Physiol. A Neuroethol. Sensory, Neural, Behav. Physiol. 188, 419-437.

Borst, A., Haag, J., and Reiff, D.F. (2010). Fly Motion Vision. Annu. Rev. Neurosci. 33, 49-70.

Boutros, M., Kiger, A.A., Armknecht, S., Kerr, K., Hild, M., Koch, B., Haas, S.A., Paro, R., and Perrimon, N. (2004). Genome-Wide RNAi Analysis of Growth and Viability in Drosophila Cells. Science (80-.). 303, 832-835.

Brady, N., and Field, D.J. (2000). Local contrast in natural images: Normalisation and coding efficiency. Perception 29, 1041-1055.

Braitenberg, V. (1967). Patterns of projection in the visual system of the fly. I. Retina-lamina projections. Exp. Brain Res. 3, 271-298.

Brand, A.H., and Perrimon, N. (1993). Targeted gene expression as a means of altering cell fates and generating dominant phenotypes. Development 118, 401-415.

Britt, S.G., Feiler, R., Kirschfeld, K., and Zuker, C.S. (1993). Spectral tuning of rhodopsin and metarhodopsin in vivo. Neuron 11, 29-39.

Brotz, T.M., Gundelfinger, E.D., and Borst, A. (2001). Cholinergic and GABAergic pathways in fly motion vision. BMC Neurosci. 2 .

Broussard, G.J., Liang, R., and Tian, L. (2014). Monitoring activity in neural circuits with genetically encoded indicators. Front. Mol. Neurosci. 7.

Brown and Blinks (1974). Changes in Intracellular Free Calcium Concentration during Illumination of Invertebrate Photoreceptors : Detection with Aequorin. J. Gen. Physiol. 64,643 .

Buchner, E. (1976). Elementary movement detectors in an insect visual system. Biol. Cybern. 24, 85-101.

Calvert, P.D., Govardovskii, V.I., Arshavsky, V.Y., and Makino, C.L. (2002). Two temporal phases of light adaptation in retinal rods. J Gen Physiol 119, 129-145.

Carthew, R.W., and Sontheimer, E.J. (2009). Origins and Mechanisms of miRNAs and siRNAs. Cell $136,642-655$.

Chemla, S., and Chavane, F. (2010). Voltage-sensitive dye imaging: Technique review and models. J. Physiol. Paris 104, 40-50.

Chen, T.-W., Wardill, T.J., Sun, Y., Pulver, S.R., Renninger, S.L., Baohan, A., Schreiter, E.R., Kerr, R.A., Orger, M.B., Jayaraman, V., et al. (2013). Ultrasensitive fluorescent proteins for imaging neuronal activity. Nature 499, 295-300.

Chen, Y., Akin, O., Nern, A., Tsui, C.Y.K., Pecot, M.Y., and Zipursky, S.L. (2014). Cell-type-specific labeling of synapses in vivo through synaptic tagging with recombination. Neuron.

Chiappe, M.E., Seelig, J.D., Reiser, M.B., and Jayaraman, V. (2010). Walking modulates speed sensitivity in drosophila motion vision. Curr. Biol. 20, 1470-1475.

Clandinin, T.R., and Zipursky, S.L. (2002). Making connections in the fly visual system. Neuron $35,827-841$. 
Clark, D.A., and Demb, J.B. (2016). Parallel Computations in Insect and Mammalian Visual Motion Processing. Curr. Biol. 26, R1062-R1072.

Clark, D.A., Bursztyn, L., Horowitz, M.A., Schnitzer, M.J., and Clandinin, T.R. (2011). Defining the Computational Structure of the Motion Detector in Drosophila. Neuron 70, 1165-1177.

Clark, D.A., Freifeld, L., and Clandinin, T.R. (2013). Mapping and Cracking Sensorimotor Circuits in Genetic Model Organisms. Neuron 78, 583-595.

Clark, D.A., Fitzgerald, J.E., Ales, J.M., Gohl, D.M., Silies, M.A., Norcia, A.M., and Clandinin, T.R. (2014). Flies and humans share a motion estimation strategy that exploits natural scene statistics. Nat. Neurosci. 17, 296-303.

Cleland, T.A. (1996). Inhibitory glutamate receptor channels. Mol. Neurobiol. 13, 97-136.

Cohen, B., Matsuo, V., and Raphan, T. (1977). Quantitative analysis of the velocity characteristics of optokinetic nystagmus and optokinetic after-nystagmus. J. Physiol. 270, 321-344.

Cover, T.M., and Thomas, J.A. (2006). Elements of Information Theory.

Currie, S.P., Doherty, G.H., and Sillar, K.T. (2016). Deep-brain photoreception links luminance detection to motor output in Xenopus frog tadpoles . Proc. Natl. Acad. Sci. 113, 60536058.

Damulewicz, M., Rosato, E., and Pyza, E. (2013). Circadian Regulation of the Na+/K+-Atpase Alpha Subunit in the Visual System Is Mediated by the Pacemaker and by Retina Photoreceptors in Drosophila Melanogaster. PLoS One 8.

Damulewicz, M., Loboda, A., Bukowska-Strakova, K., Jozkowicz, A., Dulak, J., and Pyza, E. (2015). Clock and clock-controlled genes are differently expressed in the retina, lamina and in selected cells of the visual system of Drosophila melanogaster. Front. Cell. Neurosci. 9.

DeYoe, E.A., and Bartlett, J.R. (1980). Rarity of luxotonic responses in cortical visual areas of the cat. Exp. Brain Res. 39, 125-132.

Dietzl, G., Chen, D., Schnorrer, F., Su, K.C., Barinova, Y., Fellner, M., Gasser, B., Kinsey, K., Oppel, S., Scheiblauer, S., et al. (2007). A genome-wide transgenic RNAi library for conditional gene inactivation in Drosophila. Nature 448, 151-156.

Douglass, J.K., and Strausfeld, N.J. (2003). Anatomical organization of retinotopic motionsensitive pathways in the optic lobes of flies. Microsc. Res. Tech. 62, 132-150.

Dowling, J.E., and Ripps, H. (1970). Visual adaptation in the retina of the skate. J. Gen. Physiol. 56, 491-520.

Dowling, J.E., and Ripps, H. (1972). Adaptation in skate photoreceptors. J. Gen. Physiol. 60, 698719.

Dubs, A., Laughlin, S.B., and Srinivasan, M. V. (1981). Single photon signals in fly photoreceptors and first order interneurones at behavioral threshold. J. Physiol. 317, 317-334.

Eichner, H., Joesch, M., Schnell, B., Reiff, D.F., and Borst, A. (2011). Internal Structure of the Fly Elementary Motion Detector. Neuron 70, 1155-1164.

Ekström, P., and Meissl, H. (1990). Neural elements in the pineal complex of the frog, rana esculenta, i: Centrally projecting neurons. Vis. Neurosci. 4, 389-397.

Elkins, T., and Ganetzky, B. (1988). The roles of potassium currents in Drosophila flight muscles. J. Neurosci. 8, 428-434.

Elkins, T., Ganetzky, B., and Wu, C.F. (1986). A Drosophila mutation that eliminates a calciumdependent potassium current. Proc. Natl. Acad. Sci. U. S. A. 83, 8415-8419.

Erickson, R.P. (2013). Common Properties of Sensory Systems. In Sensory Integration, pp. 7390.

Euler, T., Haverkamp, S., Schubert, T., and Baden, T. (2014). Retinal bipolar cells: Elementary building blocks of vision. Nat. Rev. Neurosci. 15, 507-519.

Fain, G.L. (1976). Sensitivity of toad rods: Dependence on wave-length and background illumination. J. Physiol. 261, 71-101.

Fain, G.L., Hardie, R., and Laughlin, S.B. (2010). Phototransduction and the Evolution of Photoreceptors. Curr. Biol. 20.

Fischbach, K.F., and Dittrich, A.P.M. (1989). The optic lobe of Drosophila melanogaster. I. A Golgi analysis of wild-type structure. Cell Tissue Res. 258, 441-475.

Fisher, Y.E., Leong, J.C.S., Sporar, K., Ketkar, M.D., Gohl, D.M., Clandinin, T.R., and Silies, M. 
(2015a). A Class of Visual Neurons with Wide-Field Properties Is Required for Local Motion Detection. Curr. Biol. 25, 3178-3189.

Fisher, Y.E., Silies, M., and Clandinin, T.R. (2015b). Orientation Selectivity Sharpens Motion Detection in Drosophila. Neuron 88, 390-402.

Fisher, Y.E., Yang, H.H., Isaacman-Beck, J., Xie, M., Gohl, D.M., and Clandinin, T.R. (2017). FlpStop, a tool for conditional gene control in Drosophila. Elife.

Franz, M.O., and Krapp, H.G. (2000). Wide-field, motion-sensitive neurons and matched filters for optic flow fields. Biol. Cybern. 83, 185-197.

Frohlich, A., and Meinertzhagen, I.A. (1982). Synaptogenesis in the first optic neuropile of the fly's visual system. J. Neurocytol. 11, 159-180.

Frolov, R. V., Bagati, A., Casino, B., and Singh, S. (2012). Potassium channels in drosophila: Historical breakthroughs, significance, and perspectives. J. Neurogenet. 26, 275-290.

Gálvez-Santisteban, M., Rodriguez-Fraticelli, A.E., Bryant, D.M., Vergarajauregui, S., Yasuda, T., Bañón-Rodríguez, I., Bernascone, I., Datta, A., Spivak, N., Young, K., et al. (2012). Synaptotagmin-like proteins control the formation of a single apical membrane domain in epithelial cells. Nat. Cell Biol. 14, 838-849.

Gao, S., Takemura, S. ya, Ting, C.Y., Huang, S., Lu, Z., Luan, H., Rister, J., Thum, A.S., Yang, M., Hong, S.T., et al. (2008). The Neural Substrate of Spectral Preference in Drosophila. Neuron 60, $328-342$.

Geisler, W.S. (2008). Visual Perception and the Statistical Properties of Natural Scenes. Annu. Rev. Psychol. 59, 167-192.

Gengs, C., Leung, H.T., Skingsley, D.R., Iovchev, M.I., Yin, Z., Semenov, E.P., Burg, M.G., Hardie, R.C., and Pak, W.L. (2002). The target of Drosophila photoreceptor synaptic transmission is a histamine-gated chloride channel encoded by ort (hclA). J. Biol. Chem. 277, 4211342120.

Gerschenfeld, H.M., Piccolino, M., and Neyton, J. (1980). Feed-back modulation of cone synapses by L-horizontal cells of turtle retina. J. Exp. Biol. 89, 177-192.

Glantz, R.M. (1973). Five classes of visual interneurons in the optic nerve of the hermit crab. J. Neurobiol. 4, 301-319.

Göbel, W., and Helmchen, F. (2007). In Vivo Calcium Imaging of Neural Network Function. Physiology 22, 358-365.

Gohl, D.M., Silies, M. a, Gao, X.J., Bhalerao, S., Luongo, F.J., Lin, C.-C., Potter, C.J., and Clandinin, T.R. (2011). A versatile in vivo system for directed dissection of gene expression patterns. Nat. Methods 8, 231-237.

Golic, K.G., and Lindquist, S. (1989). The FLP recombinase of yeast catalyzes site-specific recombination in the drosophila genome. Cell 59, 499-509.

Götz, K.G. (1964). Optomotorische Untersuchung des visuellen systems einiger Augenmutanten der Fruchtfliege Drosophila. Kybernetik 2, 77-92.

Greenspan, R.J., Finn, J.A., and Hall, J.C. (1980). Acetylcholinesterase mutants in drosophila and their effects on the structure and function of the cental nervous system. J. Comp. Neurol. 189, 741-774.

Gruntman, E., and Turner, G.C. (2013). Integration of the olfactory code across dendritic claws of single mushroom body neurons. Nat. Neurosci. 16, 1821-1829.

Guy, R.G., and Srinivasan, M. V. (1988). Integrative properties of second-order visual neurons: a study of large monopolar cells in the dronefly Eristalis. J. Comp. Physiol. A 162, 317-331.

Haag, J., Arenz, A., Serbe, E., Gabbiani, F., and Borst, A. (2016). Complementary mechanisms create direction selectivity in the fly. Elife 5.

Haag, J., Mishra, A., and Borst, A. (2017). A common directional tuning mechanism of Drosophila motion-sensing neurons in the ON and in the OFF pathway. Elife 6.

Hannon, G.J. (2002). [0027] RNA interference. Nature 418, 244-251.

Hardie, R.C. (1987). Is histamine a neurotransmitter in insect photoreceptors? J. Comp. Physiol. A 161, 201-213.

Hardie, R.C. (1989). A histamine-activated chloride channel involved in neurotransmission at a photoreceptor synapse. Nature 339, 704-706. 
Hardie, R.C. (2011). Functional Organization of the Fly Retina. pp. 1-79.

Hardie, R.C. (2012). Phototransduction mechanisms in Drosophila microvillar photoreceptors. Wiley Interdiscip. Rev. Membr. Transp. Signal. 1, 162-187.

Hardie, R.C., and Postma, M. (2008). Phototransduction in microvillar photoreceptors of.

Hardie, R.C., and Raghu, P. (2001). Visual transduction in Drosophila. Nature 413, 186-193.

Hardie, R.C., and Weckström, M. (1990). Three classes of potassium channels in large monopolar cells of the blowfly Calliphora vicina. J. Comp. Physiol. A 167, 723-736.

Hardie, R.C., Franceschini, N., Ribi, W., and Kirschfeld, K. (1981). Distribution and properties of sex-specific photoreceptors in the fly Musca domestica. J. Comp. Physiol. 145, 139-152.

Harris, W.A., Stark, W.S., and Walker, J.A. (1976). Genetic dissection of the photoreceptor system in the compound eye of Drosophila melanogaster. J. Physiol. 256, 415-439.

Hassenstein, B. (1951). Ommatidienraster und afferente Bewegungsintegration. J. Comp. Physiol. A Neuroethol. Sensory, Neural, Behav. Physiol. 33, 301-326.

Hassenstein, B., and Reichardt, W. (1956). Systemtheoretische Analyse der Zeit-, Reihenfolgenund Vorzeichenauswertung bei der Bewegungsperzeption des Rüsselkäfers Chlorophanus. Zeitschrift Fur Naturforsch. - Sect. B J. Chem. Sci. 11, 513-524.

Van Hateren, J.H. (1997). Processing of natural time series of intensities by the visual system of the blowfly. Vision Res. 37, 3407-3416.

Hattar, S., Liao, H.W., Takao, M., Berson, D.M., and Yau, K.W. (2002). Melanopsin-containing retinal ganglion cells: Architecture, projections, and intrinsic photosensitivity. Science (80-. ). 295, 1065-1070.

Hausen, K. (1976). Functional Characterization and Anatomical Identification of Motion Sensitive Neurons in the Lobula plate of the Blowfly Calliphora erythrocephala. Zeitschrift Fur Naturforsch. - Sect. C J. Biosci. 31, 629-634.

Hecht, S., and Wald, G. (1934). The visual acuity and intensity discrimination of Drosophila. J. Gen. Physiol. 17, 517-547.

Heintzen, C. (2012). Plant and fungal photopigments. Wiley Interdiscip. Rev. Membr. Transp. Signal. 1, 411-432.

Heisenberg, M., and Buchner, E. (1977). The rôle of retinula cell types in visual behavior of Drosophila melanogaster. J. Comp. Physiol. A 117, 127-162.

Heisenberg, M., and Wolf, R. (1984). Vision in Drosophila: Genetics of Microbehavior.

Henderson, S.R., Reuss, H., and Hardie, R.C. (2000). Single photon responses in Drosophila photoreceptors and their regulation by Ca2+. J. Physiol. 524, 179-194.

Hengstenberg, R., Hausen, K., and Hengstenberg, B. (1982). The number and structure of giant vertical cells (VS) in the lobula plate of the blowfly Calliphora erythrocephala. J. Comp. Physiol. $\square$ A 149, 163-177.

Hodge, J.J.L., Choi, J.C., O'Kane, C.J., and Griffith, L.C. (2005). Shaw potassium channel genes in Drosophila. J. Neurobiol. 63, 235-254.

Holmes, T.C., Sheeba, V., Mizrak, D., Rubovszky, B., and Dahdal, D. (2007). Circuit-breaking and Behavioral Analysis by Molecular Genetic Manipulation of Neural Activity in Drosophila.

Honegger, H.W. (1978). Sustained and transient responding units in the medulla of the cricket Gryllus campestris. J. Comp. Physiol. $\square$ A 125, 259-266.

Hong, S.-T., Bang, S., Paik, D., Kang, J., Hwang, S., Jeon, K., Chun, B., Hyun, S., Lee, Y., and Kim, J. (2006). Histamine and Its Receptors Modulate Temperature-Preference Behaviors in Drosophila. J. Neurosci. 26, 7245-7256.

Honkanen, A., Immonen, E.V., Salmela, I., Heimonen, K., and Weckström, M. (2017). Insect photoreceptor adaptations to night vision. Philos. Trans. R. Soc. B Biol. Sci. 372.

Hotta, Y., and Benzer, S. (2006). Genetic Dissection of the Drosophila Nervous System by Means of Mosaics. Proc. Natl. Acad. Sci. 67, 1156-1163.

Hsu, C.T., and Bhandawat, V. (2016). Organization of descending neurons in Drosophila melanogaster. Sci. Rep. 6.

Hu, W., Wang, T., Wang, X., and Han, J. (2015). I<inf $>$ h</inf $>$ Channels Control Feedback Regulation from Amacrine Cells to Photoreceptors. PLoS Biol. 13.

Jackowska, M., Bao, R., Liu, Z., McDonald, E.C., Cook, T.A., and Friedrich, M. (2007). Genomic and 
gene regulatory signatures of cryptozoic adaptation: Loss of blue sensitive photoreceptors through expansion of long wavelength-opsin expression in the red flour beetle Tribolium castaneum. Front. Zool. 4.

Jaramillo, A.M., Zheng, X., Zhou, Y., Amado, D.A., Sheldon, A., Sehgal, A., and Levitan, I.B. (2004). Pattern of distribution and cycling of SLOB, Slowpoke channel binding protein, in Drosophila. BMC Neurosci. 5.

Järvilehto, M., and Zettler, F. (1971). Localized intracellular potentials from pre- and postsynaptic components in the external plexiform layer of an insect retina. Z. Vgl. Physiol. 75, 422-440.

Jenett, A., Rubin, G.M., Ngo, T.T.B., Shepherd, D., Murphy, C., Dionne, H., Pfeiffer, B.D., Cavallaro, A., Hall, D., Jeter, J., et al. (2012). A GAL4-Driver Line Resource for Drosophila Neurobiology. Cell Rep. 2, 991-1001.

Joesch, M., Plett, J., Borst, A., and Reiff, D.F. (2008). Response Properties of Motion-Sensitive Visual Interneurons in the Lobula Plate of Drosophila melanogaster. Curr. Biol. 18, 368374.

Joesch, M., Schnell, B., Raghu, S.V., Reiff, D.F., and Borst, A. (2010). ON and off pathways in Drosophila motion vision. Nature 468, 300-304.

Jonchere, V., and Bennett, D. (2013). Validating RNAi Phenotypes in Drosophila Using a Synthetic RNAi-Resistant Transgene. PLoS One 8, e70489.

Juusola, M., and Hardie, R.C. (2001). Light adaptation in Drosophila photoreceptors: I. Response dynamics and signaling efficiency at 25 degrees C. J. Gen. Physiol. 117, 3-25.

Juusola, M., Uusitalo, R.O., and Weckström, M. (1995). Transfer of graded potentials at the photoreceptor-interneuron synapse. J. Gen. Physiol. 105, 117-148.

Kalmus, H. (1943). The optomotor responses of some eye mutants of Drosophila. J. Genet. 45, 206-213.

Kamikouchi, A., Inagaki, H.K., Effertz, T., Hendrich, O., Fiala, A., Göpfert, M.C., and Ito, K. (2009). The neural basis of Drosophila gravity-sensing and hearing. Nature 458, 165-171.

Kaplan, W.D., and Trout, W.E. (1969). The behavior of four neurological mutants of Drosophila. Genetics 61, 399-409.

Kayama, Y., Riso, R., Bartlett, J.R., and Doty, R.W. (1979). Luxotoxic response of units in macaque striate cortex. J. Neurophysiol. 42, 1495-1517.

Khan, A.A., Betel, D., Miller, M.L., Sander, C., Leslie, C.S., and Marks, D.S. (2009). Transfection of small RNAs globally perturbs gene regulation by endogenous microRNAs. Nat. Biotechnol. 27, 549-555.

Kirschfeld, K. (1967). Die projektion der optischen umwelt auf das raster der rhabdomere im komplexauge von Musca. Exp. Brain Res. 3, 248-270.

Kitamoto, T. (2001). Conditional modification of behavior inDrosophila by targeted expression of a temperature-sensitiveshibire allele in defined neurons. J. Neurobiol. 47, 81-92.

Koester, H.J., and Sakmann, B. (2000). Calcium dynamics associated with action potentials in single nerve terminals of pyramidal cells in layer $2 / 3$ of the young rat neocortex. J. Physiol. 529, 625-646.

Kolodziejczyk, A., Sun, X., Meinertzhagen, I.A., and Nässel, D.R. (2008). Glutamate, GABA and acetylcholine signaling components in the lamina of the Drosophila visual system. PLoS One 3, e2110.

Krapp, H.G., and Hengstenberg, R. (1996). Estimation of self-motion by optic flow processing in single visual interneurons. Nature 384, 463-466.

Kulkarni, M.M., Booker, M., Silver, S.J., Friedman, A., Hong, P., Perrimon, N., and Mathey-Prevot, B. (2006). Evidence of off-target effects associated with long dsRNAs in Drosophila melanogaster cell-based assays. Nat. Methods 3, 833-838.

Lai, S.-L., and Lee, T. (2006). Genetic mosaic with dual binary transcriptional systems in Drosophila. Nat. Neurosci. 9, 703-709.

Land, M.F. (1990). Direct observation of receptors and images in simple and compound eyes. Vision Res. 30, 1721-1734.

Laughlin, S. (1981a). A simple coding procedure enhances a neuron's information capacity. 
Zeitschrift Fur Naturforsch. - Sect. C J. Biosci. doi:10.151, 1981-9-1040.

Laughlin, S. (1981b). Neural Principles in the Peripheral Visual Systems of Invertebrates. pp. $133-280$.

Laughlin, S.B. (1975). Receptor and Interneuron Light-adaptation in the Dragonfly Visual System. Zeitschrift Fur Naturforsch. - Sect. C J. Biosci. 30, 306-308.

Laughlin, S.B. (1989). The role of sensory adaptation in the retina. Exp Biol 146, 39-62.

Laughlin, S.B., and Hardie, R.C. (1978). Common strategies for light adaptation in the peripheral visual systems of fly and dragonfly. J. Comp. Physiol. 128, 319-340.

Laughlin, S.B., Howard, J., and Blakeslee, B. (1987). Synaptic limitations to contrast coding in the retina of the blowfly Calliphora. Proc. R. Soc. Lond. B. Biol. Sci. 231, 437-467.

Lee, T., and Luo, L. (1999). Mosaic analysis with a repressible neurotechnique cell marker for studies of gene function in neuronal morphogenesis. Neuron 22, 451-461.

Lee, T., and Luo, L. (2001). Mosaic analysis with a repressible cell marker (MARCM) for Drosophila neural development. Trends Neurosci. 24, 251-254.

Lee, Y.S., Nakahara, K., Pham, J.W., Kim, K., He, Z., Sontheimer, E.J., and Carthew, R.W. (2004). Distinct roles for Drosophila Dicer-1 and Dicer-2 in the siRNA/miRNA silencing pathways. Cell 117, 69-81.

Leonard, D.S., Bowman, V.D., Ready, D.F., and Pak, W.L. (1992). Degeneration of photoreceptors in rhodopsin mutants of Drosophila. J. Neurobiol. 23, 605-626.

Leong, J.C.S., Esch, J.J., Poole, B., Ganguli, S., and Clandinin, T.R. (2016). Direction Selectivity in Drosophila Emerges from Preferred-Direction Enhancement and Null-Direction Suppression. J. Neurosci. 36, 8078-8092.

Leonhardt, A., Meier, M., Serbe, E., Eichner, H., and Borst, A. (2017). Neural mechanisms underlying sensitivity to reverse-phi motion in the fly. PLoS One 12, e0189019.

Lin, S.W., and Sakmar, T.P. (1999). Colour Tuning Mechanisms of Visual Pigments. pp. 124-141.

Lisman, J.E., and Brown, J.E. (1975). Effects of intracellular injection of calcium buffers on light adaptation in Limulus ventral photoreceptors. J. Gen. Physiol. 66, 489-506.

Liu, W.W., and Wilson, R.I. (2013). Glutamate is an inhibitory neurotransmitter in the Drosophila olfactory system. Proc. Natl. Acad. Sci. 110, 10294-10299.

Liu, Q., Rand, T.A., Kalidas, S., Du, F., Kim, H.E., Smith, D.P., and Wang, X. (2003). R2D2, a bridge between the initiation and effector steps of the Drosophila RNAi pathway. Science (80-. ). 301, 1921-1925.

Luan, H., Peabody, N.C., Vinson, C.R.R., and White, B.H. (2006). Refined Spatial Manipulation of Neuronal Function by Combinatorial Restriction of Transgene Expression. Neuron 52, 425-436.

Luo, L. (2007). Fly MARCM and mouse MADM: Genetic methods of labeling and manipulating single neurons. Brain Res. Rev. 55, 220-227.

Luo, L., Callaway, E.M., and Svoboda, K. (2018). Genetic Dissection of Neural Circuits: A Decade of Progress. Neuron 98, 256-281.

Ma, Y., Creanga, A., Lum, L., and Beachy, P.A. (2006). Prevalence of off-target effects in Drosophila RNA interference screens. Nature 443, 359-363.

Maimon, G., Straw, A.D., and Dickinson, M.H. (2010). Active flight increases the gain of visual motion processing in Drosophila. Nat. Neurosci. 13, 393-399.

Maisak, M.S., Haag, J., Ammer, G., Serbe, E., Meier, M., Leonhardt, A., Schilling, T., Bahl, A., Rubin, G.M., Nern, A., et al. (2013). A directional tuning map of Drosophila elementary motion detectors. Nature 500, 212-216.

Mamiya, A., Gurung, P., and Tuthill, J.C. (2018). Neural Coding of Leg Proprioception in Drosophila. Neuron 100, 636-650.

Mauss, A.S., Pankova, K., Arenz, A., Nern, A., Rubin, G.M., and Borst, A. (2015). Neural Circuit to Integrate Opposing Motions in the Visual Field. Cell 162, 351-362.

Meier, M., Serbe, E., Maisak, M.S., Haag, J., Dickson, B.J., and Borst, A. (2014). Neural circuit components of the drosophila off motion vision pathway. Curr. Biol. 24, 385-392.

Meinertzhagen, I. a, and O'Neil, S.D. (1991). Synaptic organization of columnar elements in the lamina of the wild type in Drosophila melanogaster. J. Comp. Neurol. 305, 232-263. 
Meinertzhagen, I.A., and Sorra, K.E. (2001). Chapter 3 Synaptic organization in the fly's optic lamina: few cells, many synapses and divergent microcircuits. Prog. Brain Res. 131, 5369.

Michelson, A. (1972). Studies in optics (Chicago IL: University of Chicago Press).

Milner, E.S., and Do, M.T.H. (2017). A Population Representation of Absolute Light Intensity in the Mammalian Retina. Cell 171, 865-876.e16.

Mimura, K. (1974). Analysis of visual information in lamina neurones of the fly. J. Comp. Physiol. $88,335-372$.

Montell, C. (2012). Drosophila visual transduction. Trends Neurosci. 35, 356-363.

Namiki, S., Dickinson, M.H., Wong, A.M., Korff, W., and Card, G.M. (2018). The functional organization of descending sensory-motor pathways in Drosophila. Elife 7.

Nern, A., Zhu, Y., and Zipursky, S.L. (2008). Local N-Cadherin Interactions Mediate Distinct Steps in the Targeting of Lamina Neurons. Neuron 58, 34-41.

Nern, A., Pfeiffer, B.D., and Rubin, G.M. (2015). Optimized tools for multicolor stochastic labeling reveal diverse stereotyped cell arrangements in the fly visual system. Proc. Natl. Acad. Sci. 112, E2967-E2976.

Nicol, D., and Meinertzhagen, I.A. (1982). An analysis of the number and composition of the synaptic populations formed by photoreceptors of the fly. J. Comp. Neurol. 207, 29-44.

Nippe, O.M., Wade, A.R., Elliott, C.J.H., and Chawla, S. (2017). Circadian Rhythms in Visual Responsiveness in the Behaviorally Arrhythmic Drosophila Clock Mutant ClkJrk. J. Biol. Rhythms 32, 583-592.

Normann, R.A., and Werblin, F.S. (1974). Control of Retinal Sensitivity I . Light and Dark Adaptation of. J. Gen. Physiol. 63, 37-61.

Novina, C.D., and Sharp, P.A. (2004). The RNAi revolution. Nature 430, 161-164.

O'Carroll, D.C., and Warrant, E.J. (2017). Vision in dim light: highlights and challenges. Philos. Trans. R. Soc. B Biol. Sci. 372, 20160062.

O’Tousa, J.E., Baehr, W., Martin, R.L., Hirsh, J., Pak, W.L., and Applebury, M.L. (1985). The Drosophila ninaE gene encodes an opsin. Cell 40, 839-850.

Odermatt, B., Nikolaev, A., and Lagnado, L. (2012). Encoding of Luminance and Contrast by Linear and Nonlinear Synapses in the Retina. Neuron 73, 758-773.

Oesch, N.W., and Diamond, J.S. (2011). Ribbon synapses compute temporal contrast and encode luminance in retinal rod bipolar cells. Nat. Neurosci. 14, 1555-1561.

Pak, W.L., Grossfield, J., and Arnold, K.S. (1970). Mutants of the visual pathway of drosophila melanogaster. Nature 227, 518-520.

Pantazis, A., Segaran, A., Liu, C.-H., Nikolaev, A., Rister, J., Thum, A.S., Roeder, T., Semenov, E., Juusola, M., and Hardie, R.C. (2008). Distinct Roles for Two Histamine Receptors (hclA and hclB) at the Drosophila Photoreceptor Synapse. J. Neurosci. 28, 7250-7259.

Park, E.J., and Wasserman, S.M. (2018). Diversity of visuomotor reflexes in two Drosophila species. Curr. Biol. 28, R865-R866.

Peng, J., Santiago, I.J., Ahn, C., Gur, B., Tsui, C.K., Su, Z., Xu, C., Karakhanyan, A., Silies, M., and Pecot, M.Y. (2018). Drosophila fezf coordinates laminar-specific connectivity through cell-intrinsic and cell-extrinsic mechanisms. Elife 7, e33962.

Pfeiffer, B.D., Ngo, T.T.B., Hibbard, K.L., Murphy, C., Jenett, A., Truman, J.W., and Rubin, G.M. (2010). Refinement of tools for targeted gene expression in Drosophila. Genetics 186, 735-755.

Pickard, G.E., and Sollars, P.J. (2012). Intrinsically photosensitive retinal ganglion cells. Rev. Physiol. Biochem. Pharmacol. 162, 59-90.

Pilot, F., Philippe, J.M., Lemmers, C., and Lecuit, T. (2006). Spatial control of actin organization at adherens junctions by the synaptotagmin-like protein Btsz. Nature 442, 580-584.

Posnien, N., Hopfen, C., Hilbrant, M., Ramos-Womack, M., Murat, S., Schönauer, A., Herbert, S.L., Nunes, M.D.S., Arif, S., Breuker, C.J., et al. (2012). Evolution of eye morphology and Rhodopsin expression in the Drosophila melanogaster species subgroup. PLoS One 7, e37346.

Potter, C.J., Tasic, B., Russler, E. V., Liang, L., and Luo, L. (2010). The Q system: A repressible 
binary system for transgene expression, lineage tracing, and mosaic analysis. Cell 141, 536-548.

Prelich, G. (2012). Gene overexpression: Uses, mechanisms, and interpretation. Genetics 190, 841-854.

Ratliff, C.P., Borghuis, B.G., Kao, Y.-H., Sterling, P., and Balasubramanian, V. (2010). Retina is structured to process an excess of darkness in natural scenes. Proc. Natl. Acad. Sci. 107, 17368-17373.

Reiff, D.F., Plett, J., Mank, M., Griesbeck, O., and Borst, A. (2010). Visualizing retinotopic halfwave rectified input to the motion detection circuitry of Drosophila. Nat. Neurosci. 13, 973-978.

Riabinina, O., and Potter, C.J. (2016). The q-system: A versatile expression system for drosophila. In Methods in Molecular Biology, pp. 53-78.

Richter, F.G., Fendl, S., Haag, J., Drews, M.S., and Borst, A. (2018). Glutamate Signaling in the Fly Visual System. IScience 7, 85-95.

Rieke, F., and Rudd, M.E. (2009). The Challenges Natural Images Pose for Visual Adaptation. Neuron 64, 605-616.

Riemensperger, T., Pech, U., Dipt, S., and Fiala, A. (2012). Optical calcium imaging in the nervous system of Drosophila melanogaster. Biochim. Biophys. Acta - Gen. Subj. 1820, 11691178.

Rister, J., Pauls, D., Schnell, B., Ting, C.Y., Lee, C.H., Sinakevitch, I., Morante, J., Strausfeld, N.J., Ito, K., and Heisenberg, M. (2007). Dissection of the Peripheral Motion Channel in the Visual System of Drosophila melanogaster. Neuron 56, 155-170.

Rivera-Alba, M., Vitaladevuni, S.N., Mischenko, Y., Lu, Z., Takemura, S.Y., Scheffer, L., Meinertzhagen, I.A., Chklovskii, D.B., and De Polavieja, G.G. (2011). Wiring economy and volume exclusion determine neuronal placement in the Drosophila brain. Curr. Biol. 21, 2000-2005.

Rusanen, J., and Weckström, M. (2016). Frequency-selective transmission of graded signals in large monopolar neurons of blowfly Calliphora vicina compound eye. J. Neurophysiol. $115,2052-2064$.

Ryglewski, S., and Duch, C. (2009). Shaker and Shal Mediate Transient Calcium-Independent Potassium Current in a Drosophila Flight Motoneuron . J. Neurophysiol. 102, 3673-3688.

Salcedo, E., Huber, a, Henrich, S., Chadwell, L. V, Chou, W.H., Paulsen, R., and Britt, S.G. (1999). Blue- and green-absorbing visual pigments of Drosophila: ectopic expression and physiological characterization of the R8 photoreceptor cell-specific Rh5 and Rh6 rhodopsins. J. Neurosci. 19, 10716-10726.

Schnaitmann, C., Haikala, V., Abraham, E., Oberhauser, V., Thestrup, T., Griesbeck, O., and Reiff, D.F. (2018). Color Processing in the Early Visual System of Drosophila. Cell 172, 318318.e18.

Schwarz, T.L., Tempel, B.L., Papazian, D.M., Jan, Y.N., and Jan, L.Y. (1988). Multiple potassiumchannel components are produced by alternative splicing at the Shaker locus in Drosophila. Nature 331, 137-142.

Serano, J., and Rubin, G.M. (2003). The Drosophila synaptotagmin-like protein bitesize is required for growth and has mRNA localization sequences within its open reading frame. Proc. Natl. Acad. Sci. 100, 13368-13373.

Serbe, E., Meier, M., Leonhardt, A., and Borst, A. (2016). Comprehensive Characterization of the Major Presynaptic Elements to the Drosophila OFF Motion Detector. Neuron 89, 829841.

Shahidullah, M., Reddy, S., Fei, H., and Levitan, I.B. (2009). In Vivo Role of a Potassium ChannelBinding Protein in Regulating Neuronal Excitability and Behavior. J. Neurosci. 29, 13328-13337.

Shapley, R., and Enroth-Cugell, C. (1984). Chapter 9 Visual adaptation and retinal gain controls. Prog. Retin. Res. 3, 263-346.

Shinomiya, K., Karuppudurai, T., Lin, T.Y., Lu, Z., Lee, C.H., and Meinertzhagen, I.A. (2014). Candidate neural substrates for off-edge motion detection in drosophila. Curr. Biol. 24, 
1062-1070.

Silies, M., Gohl, D., Fisher, Y., Freifeld, L., Clark, D., and Clandinin, T.R. (2013). Modular Use of Peripheral Input Channels Tunes Motion-Detecting Circuitry. Neuron 79, 111-127.

Silies, M., Gohl, D.M., and Clandinin, T.R. (2014). Motion-Detecting Circuits in Flies: Coming into View. Annu. Rev. Neurosci. 37, 307-327.

St-Pierre, F., Marshall, J.D., Yang, Y., Gong, Y., Schnitzer, M.J., and Lin, M.Z. (2014). High-fidelity optical reporting of neuronal electrical activity with an ultrafast fluorescent voltage sensor. Nat. Neurosci. 17, 884-889.

Stavenga, D.G. (1979). Pseudopupils of Compound Eyes. (Comparative Physiology and Evolution of Vision in Invertebrates), pp. 357-439.

Stavenga, D.G., and Arikawa, K. (2008). One Rhodopsin per Photoreceptor: Iro-C Genes Break the Rule. PLoS Biol. 6, e115.

Stavenga, D.G., Smits, R.P., and Hoenders, B.J. (1993). Simple exponential functions describing the absorbance bands of visual pigment spectra. Vision Res. 33, 1011-1017.

Stöckl, A.L., O'Carroll, D.C., and Warrant, E.J. (2016). Neural summation in the hawkmoth visual system extends the limits of vision in dim light. Curr. Biol. 26, 821-826.

Strother, J.A., Wu, S.T., Wong, A.M., Nern, A., Rogers, E.M., Le, J.Q., Rubin, G.M., and Reiser, M.B. (2017). The Emergence of Directional Selectivity in the Visual Motion Pathway of Drosophila. Neuron 94, 168-182.

Struhl, G., and Basler, K. (1993). Organizing activity of wingless protein in Drosophila. Cell 72, 527-540.

Suh, B., and Baccus, S.A. (2014). Building Blocks of Temporal Filters in Retinal Synapses. PLoS Biol. 12.

Suver, M.P., Huda, A., Iwasaki, N., Safarik, S., and Dickinson, M.H. (2016). An Array of Descending Visual Interneurons Encoding Self-Motion in Drosophila . J. Neurosci. 36, 11768-11780.

Takemura, S., Bharioke, A., Lu, Z., Nern, A., Vitaladevuni, S., Rivlin, P.K., Katz, W.T., Olbris, D.J., Plaza, S.M., Winston, P., et al. (2013). A visual motion detection circuit suggested by Drosophila connectomics. Nature 500, 175-181.

Takemura, S. ya, Nern, A., Chklovskii, D.B., Scheffer, L.K., Rubin, G.M., and Meinertzhagen, I.A. (2017). The comprehensive connectome of a neural substrate for ' $O N$ ' motion detection in Drosophila. Elife 6, e24394.

Takemura, S.Y., Lu, Z., and Meinertzhagen, I.A. (2008). Synaptic circuits of the Drosophila optic lobe: The input terminals to the medulla. J. Comp. Neurol. 509, 493-513.

Tammero, L.F., and Dickinson, M.H. (2002). Collision-avoidance and landing responses are mediated by separate pathways in the fruit fly, Drosophila melanogaster. J. Exp. Biol. 205, 2785-2798.

Tan, L., Zhang, K.X., Pecot, M.Y., Nagarkar-Jaiswal, S., Lee, P.T., Takemura, S.Y., McEwen, J.M., Nern, A., Xu, S., Tadros, W., et al. (2015). Ig Superfamily Ligand and Receptor Pairs Expressed in Synaptic Partners in Drosophila. Cell 163, 1756-1769.

Terakita, A. (2005). The opsins. Genome Biol. 6.

Theis, L., Berens, P., Froudarakis, E., Reimer, J., Román Rosón, M., Baden, T., Euler, T., Tolias, A.S., and Bethge, M. (2016). Benchmarking Spike Rate Inference in Population Calcium Imaging. Neuron 90, 471-482.

Timofeev, K., Joly, W., Hadjieconomou, D., and Salecker, I. (2012). Localized netrins act as positional cues to control layer-specific targeting of photoreceptor axons in drosophila. Neuron 75, 80-93.

Tsytsarev, V., Liao, L.-D., Kong, K.V., Liu, Y.-H., Erzurumlu, R.S., Olivo, M., and Thakor, N. V. (2014). Recent Progress in Voltage-Sensitive Dye Imaging for Neuroscience. J. Nanosci. Nanotechnol. 14, 4733-4744.

Tuthill, J.C., Nern, A., Holtz, S.L., Rubin, G.M., and Reiser, M.B. (2013). Contributions of the 12 Neuron Classes in the Fly Lamina to Motion Vision. Neuron 79, 128-140.

Uusitalo, R.O., Juusola, M., and Weckström, M. (1995). Graded responses and spiking properties of identified first-order visual interneurons of the fly compound eye. J. Neurophysiol. 73, 1782-1792. 
Venken, K.J.T., Simpson, J.H., and Bellen, H.J. (2011). Genetic manipulation of genes and cells in the nervous system of the fruit fly. Neuron 72, 202-230.

Vinayak, P., Coupar, J., Hughes, S.E., Fozdar, P., Kilby, J., Garren, E., Yoshii, T., and Hirsh, J. (2013). Exquisite Light Sensitivity of Drosophila melanogaster Cryptochrome. PLoS Genet. 9.

Vogt, K., and Kirschfeld, K. (1984). Chemical identity of the chromophores of fly visual pigment. Naturwissenschaften 71, 211-213.

Warbington, L.C., Hillman, T., Adams, C., and Stern, M. (1996). Reduced transmitter release conferred by mutations in the slowpoke-encoded Ca2+-activated $\mathrm{K}+$ channel gene of Drosophila. Invertebr. Neurosci. 2, 51-60.

Wardill, T.J., List, O., Li, X., Dongre, S., McCulloch, M., Ting, C.-Y., O’Kane, C.J., Tang, S., Lee, C.-H., Hardie, R.C., et al. (2012). Multiple Spectral Inputs Improve Motion Discrimination in the Drosophila Visual System. Science (80-. ). 336, 925-931.

Warmke, J.W., and B, G. (1994). A family of potassium channel genes related to eag in Drosophila and mammals. Proc. Natl. Acad. Sci. U. S. A. 91, 3438-3442.

Warrant, E.J. (2017). The remarkable visual capacities of nocturnal insects: Vision at the limits with small eyes and tiny brains. Philos. Trans. R. Soc. B Biol. Sci. 372, 20160063.

Warrant, E.J., and McIntyre, P.D. (1992). The Trade-Off Between Resolution and Sensitivity in Compound Eyes. In Nonlinear Vision: Determination of Neural Receptive Fields, Function, and Networks, (Boca Raton, FL: CRC Press Inc), pp. 391-421.

Warrant, E.J., and McIntyre, P.D. (1993). Arthropod eye design and the physical limits to spatial resolving power. Prog. Neurobiol. 40, 413-461.

Warrant, E.J., Kelber, A., Gislén, A., Greiner, B., Ribi, W., and Wcislo, W.T. (2004). Nocturnal vision and landmark orientation in a tropical halictid bee. Curr. Biol. 14, 1309-1318.

Weng, M., Golden, K.L., and Lee, C.Y. (2010). dFezf/Earmuff Maintains the Restricted Developmental Potential of Intermediate Neural Progenitors in Drosophila. Dev. Cell 18, 126-135.

Witte, I., Kreienkamp, H.J., Gewecke, M., and Roeder, T. (2002). Putative histamine-gated chloride channel subunits of the insect visual system and thoracic ganglion. J. Neurochem. 83, 504-514.

Wolfgang, W.J., and Forte, M.A. (1989). Expression of acetylcholinesterase during visual system development in Drosophila. Dev. Biol. 131, 321-330.

Wu, S.M. (1991). Input-output relations of the feedback synapse between horizontal cells and cones in the tiger salamander retina. J. Neurophysiol. 65, 1197-1206.

Yang, H.H., and Clandinin, T.R. (2018). Elementary Motion Detection in Drosophila : Algorithms and Mechanisms. Annu. Rev. Vis. Sci. 4, 143-163.

Yang, H.H., and St-Pierre, F. (2016). Genetically Encoded Voltage Indicators: Opportunities and Challenges. J. Neurosci. 36, 9977-9989.

Yang, H.H.H., St-Pierre, F., Sun, X., Ding, X., Lin, M.Z.Z., and Clandinin, T.R.R. (2016). Subcellular Imaging of Voltage and Calcium Signals Reveals Neural Processing In Vivo. Cell 166, $245-257$.

Yau, K.W., and Hardie, R.C. (2009). Phototransduction Motifs and Variations. Cell 139, 246-264.

Ying, S.-Y. (2013). MicroRNA Protocols.

Yorozu, S., Wong, A., Fischer, B.J., Dankert, H., Kernan, M.J., Kamikouchi, A., Ito, K., and Anderson, D.J. (2009). Distinct sensory representations of wind and near-field sound in the Drosophila brain. Nature 458, 201-205.

Yoshioka, T., Inoue, H., and Hotta, Y. (1985). Absence of phosphatidylinositol phosphodiesterase in the head of a Drosophila visual mutant, norpA (no receptor potential A). J. Biochem. 97, 1251-1254.

Zeng, Y., Lin, Y., Abundo, A., and Dudley, R. (2015). Visual ecology of directed aerial descent in first-instar nymphs of the stick insect Extatosoma tiaratum. J. Exp. Biol. 218, 2305-2314.

Zheng, L., de Polavieja, G.G., Wolfram, V., Asyali, M.H., Hardie, R.C., and Juusola, M. (2006). Feedback Network Controls Photoreceptor Output at the Layer of First Visual Synapses in Drosophila. J. Gen. Physiol. 127, 495-510.

Zuker, C.S., Cowman, A.F., and Rubin, G.M. (1985). Isolation and structure of a rhodopsin gene 
from D. melanogaster. Cell 40, 851-858. 


\section{ACKNOWLEDGEMENTS}

I would like to express my sincere gratitude to my advisor Marion Silies. I have never met a person so full of knowledge, passion and kindness. Marion, thank you for your immense support and patience. Your guidance and attitude has taught me so many things over the past few years, from setting up the experiment, analyzing data, to presenting my project in front of a big audience. Thank you for your excitement about my data even when I was not impressed by the result. Thank you for teaching me to believe in my data and present them with pride and excitement. Thank you for allowing me to find my own "research question" and thank for always being prepared to discuss about my results and help with the analysis and interpretation. You really are my role model for a scientist, mentor, and teacher and I could not ask for a better supervisor. I am beyond proud to be your first PhD student.

Besides my advisor, I would like to thank my second reviewer and member of my thesis committee: Prof. Dr. Martin Göpfert. Thank you for taking your time and knowledge to review my thesis. My sincere thank you goes also to my third thesis committee member, Prof. Dr. Nils Brose. Thank you both for your invaluable comments, suggestions and ideas and for offering your time, support and guidance throughout my PhD.

I offer my thanks to my collaborators. Thank you Dr. Matthew Pecot for sending us $d F e z f^{1}$ flies and Prof. Dr. Mathias Wernet for flies used in norpA rescue experiments. In addition, thank you Dr. Gregor Belušič and Dr. Marko Ilić for the LedSynth.

I extend my sincere thanks to all members of the lab. Madhura, it was a pleasure to work together on the same project. Thank you for many discussions and thank you for sharing your knowledge and ideas. Burak, thank you for contributing to my project and thank you for your help with the analysis and anything else I might asked you. I would like to say thank you also to Marvin for creating the important stimulus for this project and for many discussions about the contrast and luminance calculations. Luis, thank you for all the interesting ideas, critical thinking and all the help with the statistics and analysis. Thank you also for the Matlab code, which was crucial for the subsequent analysis. Sebastian, although you are working on a different project, thank you for always showing your excitement about my project. Many thanks go also to my office partner 
Miriam. Thank you for solving my many Matlab-related questions and for many science and non-science related discussions. My sincere thanks go also to our lab technicians Christine and Jonas. Christine, thank you for teaching me fly husbandry and dissections and for taking care of my flies when I was away. Jonas, thank you for taking care of our fly stocks and making sure that they look happy and healthy. Many thanks go also to some students, which helped creating a great work environment in which I enjoyed working every day. I am very grateful for the privilege of being part of this team.

I gratefully acknowledge the funding received from the project SFB889: Cellular Mechanisms of Sensory Processing and from GGNB Junior Group Stipend. I take this opportunity to sincerely acknowledge my home institution, European Neuroscience Institute, for providing a great work place.

Last but not least, I would like to express my gratitude to my family for their constant support and encouragement. I am extremely grateful to my grandparents for being my role models and showing me the passion for animals, nature and science. I wish to extend a huge thank you to a very special person, Jan-Christian, for his love, support and understanding during my pursuit of the $\mathrm{PhD}$ degree. 